\title{
UNIVERSITY OF CALIFORNIA \\ SANTA CRUZ
}

THE STRUCTURE OF MAMMALIAN FOOD-WEBS:

INTERPRETING, PREDICTING, AND INFORMING ESTIMATES

OF SPECIES INTERACTIONS IN PALEONTOLOGICAL AND

MODERN COMMUNITIES

A dissertation submitted in partial satisfaction of the

requirements for the degree of

DOCTOR OF PHILOSOPHY

in

ECOLOGY AND EVOLUTIONARY BIOLOGY

by

Justin D. Yeakel

June 2012

The Dissertation of Justin D. Yeakel is approved:
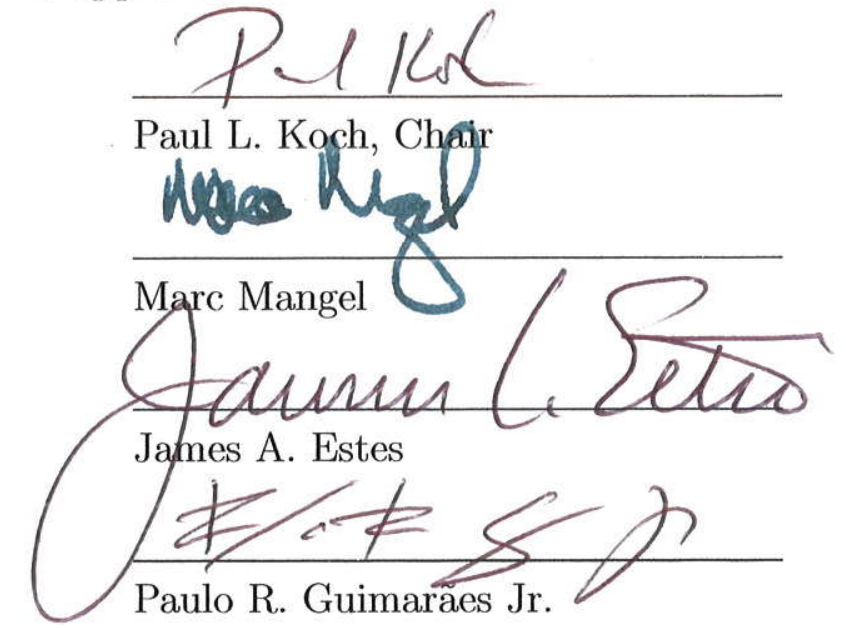

Tyrus Miller

Vice Provost and Dean of Graduate Studies 
Copyright (c) by

Justin D. Yeakel

2012 


\section{Table of Contents}

List of Figures $\quad$ vi

List of Tables $\quad$ xvi

$\begin{array}{ll}\text { Abstract } & \text { xvii }\end{array}$

Dedication $\quad$ xix

$\begin{array}{ll}\text { Acknowledgments } & \mathrm{xx}\end{array}$

1 Introduction 1

1.1 Figures ............................. 8

2 Probabilistic patterns of interaction: The effects of link-strength vari$\begin{array}{ll}\text { ance on food-web structure } & \mathbf{1 0}\end{array}$

2.1 Introduction . . . . . . . . . . . . . . . . . . . 11

2.2 Methods . . . . . . . . . . . . . . . . . . 14

2.2.1 Strength variance in food-web networks . . . . . . . . . . . 14

2.2.2 Quantifying link-strength and variance in empirical systems . . . 15

2.3 Analysis and Discussion . . . . . . . . . . . . . . . 18

2.3.1 Estimating the NLID from limited observations . . . . . . . . 20

2.3.2 Assessing structure from the NLID . . . . . . . . . . . . . . 21

2.3.3 Body size constraints and ecological implications . . . . . . . . 26

2.4 Figures . . . . . . . . . . . . . . . . . . . . . . 31

2.5 Supplementary Material . . . . . . . . . . . . . . . . 37

2.5.1 Appendix 2.1. Isotopic systems and mixing model results . . . . 37

2.5.2 Appendix 2.2. Calculation of similarity . . . . . . . . . . 38

2.5.3 Appendix 2.3. Calculation of consumer dietary specialization . . 39

2.5 .4 Tables . . . . . . . . . . . . . . . . . . 41

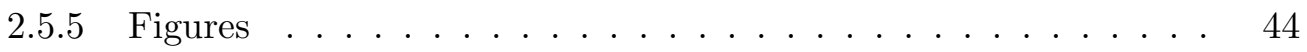


3 The impact of climate change on the structure of Pleistocene mam$\begin{array}{ll}\text { moth steppe food-webs } & \mathbf{5 0}\end{array}$

3.1 Introduction . . . . . . . . . . . . . . . . . 52

3.2 Materials and Methods . . . . . . . . . . . . . . . 54

3.2.1 Study sites . . . . . . . . . . . . . . . . 54

3.2.2 Estimating diet from stable isotope data . . . . . . . . . . 55

3.2.3 Statistical comparisons among sites . . . . . . . . . . . . . 59

3.2.4 Predator specialization and prey selectivity . . . . . . . . 60

3.2.5 Community-level network analysis . . . . . . . . . . . . 62

3.3 Results........................... 64

3.4 Discussion . . . . . . . . . . . . . . . . . . . . 67

3.4.1 Spatio-temporal patterns of species interaction . . . . . . . 68

3.4.2 Spatio-temporal patterns of community organization . . . . . . 70

3.4.3 Linking species interactions to large-scale community structure . 71

3.5 Figures . . . . . . . . . . . . . . . . . 76

3.6 Supplementary Material . . . . . . . . . . . . . . . . . 81

3.6.1 Appendix 3.1. Link-strength posterior probability distributions . 81

3.6.2 Appendix 3.2. Structural metrics . . . . . . . . . . . . . . 82

3.6.3 Figures ....................... 84

4 Foraging behavior and the constraints of enamel: Using a processbased model to predict the dietary benefits of mechanical advantage $\begin{array}{ll}\text { and megadont dentition among hominins } & 91\end{array}$

4.1 Introduction . . . . . . . . . . . . . . . . . . . . 93

4.2 Methods . . . . . . . . . . . . . . . . 96

4.2.1 Model architecture . . . . . . . . . . . . . . . . 996

4.2 .2 State dynamics . . . . . . . . . . . . . . . . . . . . . . . . 101

4.2.3 Aggregation of food resources . . . . . . . . . . . . . . . 104

4.3 Results and Discussion . . . . . . . . . . . . . . . . . . 109

4.3.1 Fitness-maximizing foraging decisions . . . . . . . . . . . . . 110

4.3.2 Informing paleo-dietary evidence . . . . . . . . . . . . . 117

4.3.3 Updating dietary information with process-based models . . . . . 120

4.3 .4 Conclusions . . . . . . . . . . . . . . . . . 122

4.4 Tables . . . . . . . . . . . . . . . . . . . . . . . . 123

4.5 Figures . . . . . . . . . . . . . . . . . . . . . . . 124

4.6 Supplementary Material . . . . . . . . . . . . . . . . . . 130

4.6.1 Appendix 4.1. Modeling $\delta^{13} \mathrm{C}$ values of simulated foragers . . . . 130

4.6 .2 Figures . . . . . . . . . . . . . . . . . 132

5 Merging resource availability with isotope mixing models: The role of neutral interaction assumptions $\quad \mathbf{1 3 5}$

5.1 Introduction . . . . . . . . . . . . . . . . . 137

5.1 .1 Background . . . . . . . . . . . . . . . 137 
5.1 .2 Incorporating biological information . . . . . . . . . . . . 139

5.1.3 Post-hoc adjustments vs. the formulation of prior distributions . 140

5.2 Methods . . . . . . . . . . . . . . . . . . 143

5.2.1 Weighting of Bayesian isotope mixing models . . . . . . . . . . 143

5.2.2 Application to a New Zealand intertidal food web . . . . . . . . 146

5.2.3 Observational estimates of prey contributions . . . . . . . . . . 147

5.2.4 Stable isotope estimates of prey contributions . . . . . . . . . . 149

5.3 Results . . . . . . . . . . . . . . . . . 150

5.3.1 Hypothetical dataset and model assessment . . . . . . . . . . 150

5.3.2 New Zealand dataset and model validation . . . . . . . . . . . . 152

5.4 Discussion . . . . . . . . . . . . . . . . . . . . 153

5.5 Tables . . . . . . . . . . . . . . . . . . 157

5.6 Figures . . . . . . . . . . . . . . . . . . . . 158

5.7 Supplementary Material . . . . . . . . . . . . . . . . . 161

5.7 .1 Figures . . . . . . . . . . . . . . . . . . 161

6 Synthesis $\quad 164$

$\begin{array}{ll}\text { Bibliography } & 169\end{array}$ 


\section{List of Figures}

1.1 A. The predation of a prey by a predator. The arrow represents the flow of biomass such that the top node denotes the predator and the lower node represents the prey. B. The predation of two prey by a single predator. If the prey are preferred similarly by the predator, this structure results in asynchronous oscillations of prey abundance (apparent competition). See panel C. for a scenario where one prey is preferred over another. C. A tri-trophic system, where a top-predator consumes a meso-predator, which in turn consumes two prey. The sizes of the nodes denote relative abundance, and the thickness of the arrows denote the magnitude of the biomass flow. The top-predator in the leftmost network is relatively abundant, depressing the meso-predator population, thereby limiting the meso-predator's effect on the two prey populations. The two rightmost networks (boxed) have reduced top-predator populations. In both cases, the meso-predator has greater abundance due to decreased predation pressure. However, in one scenario, both prey are preferred equally, resulting in apparent competition dynamics. In the second scenario, one prey is preferred over another, resulting in an asymmetric cascade. Such preferences could result from specific anatomical constraints of the meso-predator, a scenario explored in Chapter 4. . .

1.2 Distribution of Thesis Chapters as a function of the temporal range and the scale at which interactions are considered. Chapter 4 is not included because it is a methodological report, and is applicable to any time period and multiple scales of interaction. From left to right, silhouettes represent a food-web network, the Pleistocene species Bison bison and Homotherium serum, and the Plio-Pleistocene hominin Paranthropus ro-

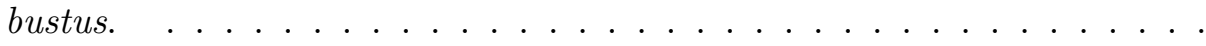


2.1 A. In this network subgraph, $\mathcal{C}_{1}$ and $\mathcal{C}_{2}$ are consumers, while $r_{1}$ and $r_{2}$ are resources. The proportional contributions of $r_{1}$ and $r_{2}$ to the diet of predator $\mathcal{C}_{1}$ are represented by the point estimates $x$ and $1-x$, for links $w_{11}$ and $w_{12}$, respectively. Because $\mathcal{C}_{2}$ is only linked to $r_{2}$ at link $w_{22}$, $r_{2}$ represents the entire diet of $\mathcal{C}_{2}$. B. Weights are illustrated as probability distributions $p_{1}(x)$ and $p_{2}(x)$. Individual draws from $p_{1}(x)$ and $p_{2}(x)$ must sum to unity. C. The variance among links of a given foodweb can be represented by the Network-Level Interaction Distribution (NLID), which is characteristic of the entire food-web. D. The variance within a link of a given food-web is represented by Pairwise Interaction Distributions (PIDs), which are unique to each link. . . . . . . . .

2.2 A. The variance among links of an empirical food-web (with 2 predators: $\mathcal{C}_{1}$ and $\mathcal{C}_{2}$, and 4 prey: $r_{1}-r_{4}$ ) is represented by both pairwise interaction distributions for each link, and a network-level interaction distribution for the system. Link thickness represents the median link-strength, shown with the vertical bar in each pairwise interaction distribution. B-C. Both model food-webs $X$ and $Y$ have link-strengths drawn from the NLID. In model food-web $X$, the weights are distributed similar to the median weights of the empirical web (i.e. equivalent structure), but they are distributed differently among nodes (i.e. alternative interactions). In model $Y$, the weight structure is different than that of the empirical web (i.e. alternative structure), and necessarily, the distribution of weights among nodes differs as well (i.e. alternative interactions). Dotted nodes indicate those with link-strengths that differ from the median link-strengths of the empirical food-web. D-F. Given a single niche axis based on a continuous resource trait (e.g. body size) where prey are ranked from $r_{1}$ to $r_{4}$, distributions of resource use are shown for the two consumers. Representations of dietary overlap illustrate higher similarity in diet between predators of the empirical system and model $X$, whereas the consumers in model $Y$ have little dietary overlap, despite sharing the same NLID.

2.3 A. Numerical results of the absolute difference in means of the true and estimated NLIDs vs. the number of PIDs sampled. The size of the theoretical food-webs are measured by the number of links, and denoted by the color gradient. B. The $\mathrm{y}$-axis is as in panel A., and the $\mathrm{x}$-axis denotes the proportion of PIDs sampled across model food-webs of varying size. Exponential curves $\left(y=a \mathrm{e}^{b x}\right)$ were fitted to binned numerical results, where each bin represents an increase in ecosystem size by 100 links. C. Numerical results of the absolute difference in standard deviation of the true and estimated NLIDs vs. the number of PIDs sampled. D. The $\mathrm{y}$-axis is as in $\mathrm{C}$; the $\mathrm{x}$-axis and exponential curves are plotted as in B. See Table S2.2 for estimated values of $a$ and $b$ for each model fit. . . . 
2.4 A. A nested pattern of interaction, where the consumers (black circles) towards the right consume prey (white circles) that are subsets of the consumers towards the left. B. A network square (circled), with four nodes connected to four links. Compartmentalization is both a function of the average density of squares relative to a fully connected network, and network connectance. In this example, at high cutoff values, the density of squares $\rightarrow 0$, while connectance $>0$, resulting in a modularity value of -1 (see Eq. 3.2). C. Two network components and an isolated node (stippled). D. Network Subgraphs 2 through 7. Subgraph 1 is represented by 2 predators and 2 prey not connected by interactions (not shown). . . . . . . . . . . . . . . . . .

2.5 A-C. Sensitivity analyses of the Saskatchewan, Amboseli, and Lake Naivasha food-web ensembles, respectively. Similarity values for each cutoff $\left(s_{i}\right)$ for Model 2 are on the $\mathrm{y}$-axis, and the $\mathrm{x}$-axis ranges from a lower standard deviation (SD) than the empirical SD to a higher SD (such that the empirical SD is central to the range; stippled vertical line). The colored data points represent the median similarity indices for each cutoff value (legend), while the top and bottom whiskers denote the $25^{\text {th }}$ and $75^{\text {th }}$ percentiles, respectively. The underlined values mark the standard deviation corresponding to the highest average similarity across cutoff values. For all systems, the highest average similarity is close to or exactly matches the empirical standard deviation. D-F. Distributions of consumer specialization for low, optimal, and high SD (lines), and for the empirical consumers (histograms), for Saskatchewan, Amboseli, and Naivasha, respectively. When NLID SD is altered to low values, the model food-web is populated with generalist consumers. When NLID SD is altered to high values, the food-web is populated with intermediate to strong specialists. When NLID SD is held at the optimal value, consumers with intermediate values are most common. . . . . . . . . . . . .

2.6 First row: the difference $(\Delta)$ in nestedness (empirical - model values) for A. Saskatchewan, Canada, B. Amboseli, Kenya, and C. Lake Naivasha, Kenya vs. cutoff values 0.1 to 0.5 . Second row: the difference $(\Delta)$ in modularity (empirical - model values) for D. Saskatchewan, Canada, E. Amboseli, Kenya, and F. Lake Naivasha, Kenya vs. cutoff values 0.1 to 0.5. Low cutoff values correspond to food-webs with both weak and strong interactions; high cutoff values correspond to the same food-webs with only the strong interactions intact. Difference values calculated using Model 1 are colored red; difference values calculated using Model 2 are colored blue. Whiskers denote the root mean square of the empirical and model standard deviations. The dotted line denotes the value at which there is no difference between the empirical and theoretical model nestedness or modularity values; the network illustration demonstrates the elimination of weak links as cutoff values increase. . . . . . . . . 
2.1 An illustration of the proportional contribution to diet matrix $\mathbf{W}_{j}$ for predator $j$. Each column $\mathbf{w}_{j k}$ is a vector that describes numerically a single PID in the probabilistic network. Equivalently, columns represent the probabilistic link-strength distribution connecting the predator $j$ to prey $k$, and account for variability within a single link. By contrast, each row is an independent draw from a Bayesian isotope mixing model, representing a single set of link-strengths for a potential quantitative food-web in the probabilistic ensemble. Because the rows describe a potential set of values for the proportional contribution of prey $k=1$ to 4 , they are constrained to sum to one. As a whole, the matrix $\mathbf{W}_{j}$ is a numeric description of a multivariate posterior probability distribution, the columns of which represent marginal distributions. . . . . . . .

2.2 Proportional contribution of prey to the diet of predators (PIDs) in the Saskatchewan system. PID estimates are from the Bayesian isotope mixing model MixSIR. Predator:prey PIDs that are not reported indicate the presence of a forbidden interaction. Species marked with stars represent the larger predators in the system; topological properties were assessed with just these predators included, and are reported in Fig. S2.6. Forbidden Links: Fox, fisher, marten, and raccoon to bison, caribou, elk, moose, white-tailed deer; coyote, lynx, and wolverine to bison, caribou, moose. . . . . . . . . . . . . . . . . .

2.3 Proportional contribution of prey to the diet of predators (PIDs) in the Amboseli system. PID estimates are from the Bayesian isotope mixing model MixSIR. Predator:prey PIDs that are not reported indicate the presence of a forbidden link. Forbidden Links: cheetah to buffalo, giraffe, wildebeest, zebra. . . . . . . . . . . . . .

2.4 Proportional contribution of prey to the diet of predators (PIDs) in the Naivasha system. PID estimates are from the Bayesian isotope mixing model MixSIR. Predator:prey PIDs that are not reported indicate the presence of a forbidden link. A subset of browsers were too isotopically similar to resolve dietary information, though they had values set apart from all potential predators, suggesting minimal dietary inclusion. As such, they were binned together as a 'browser' functional group and include: Steenbok, Dik Dik, Baboon, Grey Duiker, Klipspringer, Bushbuck, and Eland. Forbidden Links: cheetah and leopard to buffalo, waterbuck,

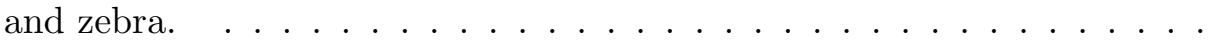


2.5 The difference $(\Delta)$ in modularity (empirical - model values) vs. the difference in nestedness for the three isotopic food-webs. Model 1 does not incorporate body size constraints, while Model 2 does incorporate body size constraints. In each system, there is a strong negative relationship between modularity and nestedness for Model 2 (though this relationship is not statistically significant for the Naivasha system). Saskatchewan Model 1: $\mathrm{R}^{2}=0.47, \mathrm{p}>0.05$, Model 2: $\mathrm{R}^{2}=0.96, \mathrm{p} \ll 0.05$. Amboseli Model 1: $\mathrm{R}^{2}=0.09 ; \mathrm{p}>0.05$, Model $2: \mathrm{R}^{2}=0.86 ; \mathrm{p}<0.05$. Naivasha Model 1: $\mathrm{R}^{2}=-0.33 ; \mathrm{p}>0.05$, Model 2: $\mathrm{R}^{2}=0.25 ; \mathrm{p}>0.05 . \quad \ldots .48$

2.6 The difference $(\Delta)$ in nestedness (empirical - model values) for Saskatchewan, Canada, with only the large predators (and predators that primarily consume larger prey) included in the food-web ensemble. Predators include: Black bear, Coyote, Lynx, Wolves, and Wolverine. These results are qualitatively similar to those obtained for the full food-web, suggesting that the structure of interactions is fairly robust to modification. . . . .

3.1 Locations of late Pleistocene mammoth steppe sites included in the analysis. The pre-, full-, and post-Glacial Beringian sites are located near Fairbanks, Alaska. Two pre- and one post-Glacial European site occur in eastern France, Belgium, and western Germany, respectively. Ber. = Beringia; Ard. = Ardennes; S.J. = Swabian Jura; J. = Jura. . . . . . .

3.2 Proportional contribution estimates of prey taxonomic groups to the diets of predator groups present in both Beringia and Europe in the pre- and post-Glacial periods (grey symbols). The contribution estimates represent numerical approximations of link-strength posterior probability distributions from the Bayesian isotope mixing model, MixSIR. Densities of contribution estimates across all prey were calculated with a kernel density algorithm $[56] . \ldots \ldots \ldots \ldots \ldots$

3.3 Predator dietary specialization $(\epsilon)$ for Beringian and European predator guilds from the pre-Glacial to the post-Glacial. A value of $\epsilon=0$ describes a generalist diet (consumption of all prey in equal amounts), whereas a value of $\epsilon=1$ describes a specialist diet (consumption of one prey to the exclusion of others). Dotted lines denote species' absence. . . . . . . .

3.4 A. The proportional contribution of each prey with respect to all potential predators in Beringia in the pre-Glacial, full-Glacial, and postGlacial. High values denote prey with higher proportional contributions across predators, whereas low values denote prey with lower proportional contributions. B. Consumer dietary specialization quantified for the predator guild $\left(\epsilon_{g}\right)$ for each time period. The median $\epsilon_{g}$ value is highest for the post-Glacial, indicating an on-average greater contribution of a smaller subset of potential prey; this trend is also observed in European systems (Fig. S3.4). Dotted lines denote species' absence. . . . . . . . . 
3.5 Modularity $(\Delta \mathcal{M})$ for A. Beringia and B. Europe across cutoff values $i$. Values $>0$ indicate that the system is more modular than would be expected given the size and predator:prey ratio of the food-web; values $<0$ indicate that the system is less modular than would be expected. The cutoff value $i=0$ refers to the whole food-web with no link deletions; cutoff values 0.1 to 0.5 describe the food-web when links $\leq 0.1$ to 0.5 are eliminated, respectively. At high cutoff values only the strongest interacting species affect the structure of the food-web. . . . . . . . .

3.1 Proportional contribution of prey to predator species in pre-, full-, and post-Glacial Beringia. Boxplots denoting the $25 \%, 50 \%$, and $75 \%$ quartiles are depicted by the black bars (the white point denotes the median value), and the underlaying distribution is depicted in grey. Bimodality is interpreted as alternative hypotheses of predator diet, where the probability of each hypothesis is determined by the density of the distribution.

3.2 Proportional contribution of prey to predator species in two pre-Glacial and one post-Glacial site in Europe. . . . . . . . . . . . . . .

3.3 The proportional contribution of prey to Neanderthal diets for the two pre-Glacial European sites: Ardennes and Swabian Jura. Two alternative dietary estimates are presented: one when a trophic discrimination factor (TDF) of $4.6 \%$ (low quality protein diet) is applied, and one when a TDF of $3.5 \% 0$ (high quality protein diet) is applied. . . . . . . . . . .

3.4 A. The proportional contribution of each prey with respect to all potential predators in Europe across the LGM. High values denote prey with higher proportional contributions across predators, whereas low values denote prey with lower proportional contributions. B. Consumer dietary specialization quantified for the predator guild $\left(\epsilon_{g}\right)$ across the LGM. The median $\epsilon_{g}$ value is highest for the post-Glacial, indicating an on-average greater contribution of a smaller subset of potential prey. . . . . . . . .

3.5 Nestedness $(\Delta \mathcal{N})$ for A. Beringia and B. Europe across cutoff values $i$. Values $>0$ indicate that the system is more modular than would be expected given the size and predator:prey ratio of the food-web; values $<0$ indicate that the system is less modular than would be expected. The cutoff value $i=0$ refers to the whole food-web with no link deletions; cutoff values 0.1 to 0.5 describe the food-web when links $\leq 0.1$ to 0.5 are eliminated, respectively. At high cutoff values only the strongest interacting species affect the structure of the food-web. . . . . . . . .

3.6 $\Delta$ Modularity for the post-Glacial Beringian food-web with a trophic discrimination factor of $\Delta^{15} \mathrm{~N}=4.6 \%$ applied. . . . . . . . . . . 
4.1 Stationary solutions to the fitness-maximizing equations $F_{\mathrm{r}, \mathrm{p}}(x, v)$ for a $50 \mathrm{~kg}$ anthropoid primate with no mechanical advantages. In rich quality habitats, fruit (green) is chosen independent of energy reserves, $x$, or enamel volume, $v$. In poor quality habitats, plant USOs (red) are chosen when energy reserves are low, and are replaced by less fracture resistant fruits as enamel volume decreases. There are no differences between wet (where the environment is primarily rich quality) or dry (where the environment is primarily poor quality) conditions. Gray elements denote values of $(x, v)$ resulting in mortality. . . . . . . . . . .

4.2 The proportional contribution of foods to the decision matrices of anthropoid primates with body sizes ranging from 10 to $70 \mathrm{~kg}$. A. Contributions of foods for the no mechanical advantage scenario. B. Contributions of foods for the arthropod mechanical advantage scenario. C. Contributions of foods for the arthropod + USO mechanical advantage scenario. Circles denote values in rich quality habitats; triangles denote values in poor quality habitats. Grass leaves are not found to be optimal foraging solutions in any decision matrix. . . . . . . . . . . . . .

4.3 Ternary diagram showing the proportional contribution of fruit, USOs, and arthropods to the decision matrices of both $50 \mathrm{~kg}$ non-megadont and megadont primates under each mechanical advantage scenario. Megadont primates have decision matrices with a greater proportion of fractureresistant, moderate calories, and low cost foods. . . . . . . . . . .

4.4 Expected fitness trajectories for $N=10050 \mathrm{~kg}$ non-megadont (solid) and megadont (stippled) primates over an estimated lifespan with varying mechanical advantages (none, arthropods, arthropods + USOs), during both wet (primarily rich quality habitat) and dry (primarily poor quality habitat) conditions. . . . . . . . . . . . . . .

4.5 Forward simulation of the $\delta^{13} \mathrm{C}$ values (black line denotes running mean; gray band denotes maximum and minimum value), mean enamel volume, and the proportional contribution of food-items to the diets of $N=100$, $50 \mathrm{~kg}$ individuals foraging in a dry environment over an estimated lifespan. A. and B. When foraging costs are minimal, a dietary switch is observed to occur near day 3500, and labels I and II denote the pre- and post-diet switch. C. and D. The same simulation when foraging costs are elevated. In panels B. and D., the red circles and triangles denote the mean encounter rate for each food in rich quality and poor quality habitats, respectively. . . . . . . . . . . . .

4.6 Stationary solutions for the fitness-maximizing equations $F_{\mathrm{r}, \mathrm{p}}(x, v)$ as a function of energy reserves $x$ and enamel volume $v$ for both non-megadont and megadont primates when grass leaves are hyper-abundant and for the arthropod + USO mechanical advantage scenario. Decision matrices are shown for both wet and dry environmental conditions. . . . . . . . . 128 
4.1 A. A cross-section of a molar. The enamel coat and dentin are colored blue and white, respectively. The average enamel thickness, $\epsilon$, is given by the average width of the enamel coat, and the dentin area, $D$, is measured by the area of dentin observed under the cross-section. B. To calculate the enamel volume of the molar, we first simplify the complex topology of the enamel coat by modeling its cross-section as a rectangle with a length equal to that of the enamel-dentin junction, $L$, and height equal to the average enamel thickness, $\epsilon$. C. Volume is calculated by modeling the enamel cross-section as a 'solid of revolution'. Total enamel volume is measured by calculating the volume of the cylinder produced when rotating the cross-section about the axis of rotation, $\phi_{r}$. This relies on the assumption that the tooth is fairly symmetrical about the axis of

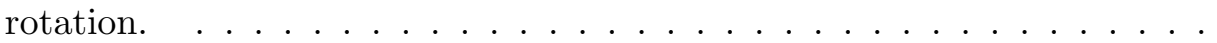

4.2 Stationary solutions for the fitness-maximizing equations $F_{\mathrm{r}, \mathrm{p}}(x, v)$ as a function of energy reserves $x$ and enamel volume $v$. Decision matrices are shown for the arthropod + USO mechanical advantage scenario. Each pair of decision matrices represent an anthropoid primate with a body size ranging from $x_{\max }=10 \mathrm{~kg}$ to $70 \mathrm{~kg}$. There are no differences between wet (primarily rich quality habitat) or dry (primarily poor quality habitat) conditions. Gray elements denote values of $(x, v)$ resulting in mortality.

4.3 Forward simulation of the $\delta^{13} \mathrm{C}$ values (black line denotes running mean; gray band denotes maximum and minimum value), mean enamel volume, and the proportional contribution of food-items to the diets of $N=100$, $50 \mathrm{~kg}$ megadont hominins foraging in wet and dry environments over an estimated lifespan. . . . . . . . . . . . . . . . .

5.1 Isotopic niche space of a theoretical single predator, four prey community. A. The predator (black) and prey (red, green, blue, orange) have a standard deviation of $(0.5,0.5)$ for both isotope tracer 1 and 2 . Two of the four prey (red and green) are isotopically distinct with no overlap. Two of the four prey (blue and orange) are isotopically identical, with $w_{i j}=1$. B. Initial MixSIR contribution-to-diet posterior probability distributions for the isotopically identical prey. C. Final weighted and renormalized posterior probability distributions for the isotopically similar prey. Relative abundance values of 0.9 and 0.1 were applied to the orange and blue prey, respectively. doi:10.1371/journal.pone.0022015.g001 . . . . . . . 
5.2 Sensitivity analysis of the weighting procedure as a function of isotopic similarity and the difference in proportional availability of isotopically similar prey. A. Sensitivity analysis of Pianka's measure of density overlap $\left(w_{i j}\right)$ across differences in mean values and variance of two bivariate normal isotopic distributions. When variance is small, $w_{i j}$ decreases sigmoidally as the difference in means increases. Larger variance predictably tends to linearize the relationship. B. The mixing space of our hypothetical scenario permits manipulation of isotopic overlap between two prey (blue and orange; the consumer is black) without altering mixing model estimates of summed proportional contribution to diet of the two prey (cf. Fig. S5.3). C. An analysis of the effect of model parameters (density overlap, $w_{i j}$, and availability differences, $\alpha_{i}-\alpha_{j}$ ) on the final weighted and renormalized posterior distributions, measured by the difference of final distribution means for blue and orange in the hypothetical isotopic dataset (Fig. 5.1). The model is assessed across all possible values of $w_{i j}$ $(0: 1)$, and across five prey availability scenarios. There is a strong linear effect of isotopic overlap on the difference in means of the weighted posterior probability distributions. The slope of this relationship is determined by availability differences. doi:10.1371/journal.pone.0022015.g001 . . .

5.3 The weighting procedure applied to a New Zealand intertidal community. A. Isotopic niche space of the New Zealand whelk- predator system, where $\delta^{13} \mathrm{C}$ is plotted on the $\mathrm{x}$-axis and $\delta^{15} \mathrm{~N}$ is plotted on the $\mathrm{y}$-axis. The $\delta^{13} \mathrm{C}$ and $\delta^{15} \mathrm{~N}$ values of the predator $\mathrm{Hs}$ are adjusted for trophic discrimination factors (see methods). B. MixSIR contributionto-diet posterior probability distributions of all New Zealand prey for predator Hs. Solid distribution density lines denote isotopically distinct prey, whereas hatched distribution density lines denote isotopically similar prey. Prey colors match those of panel 3A. C. Weighted posterior probably distributions for the isotopically similar prey. Relative abundance values of 0.05 and 0.95 correspond to $M g$ (blue) and $X p$ (green), respectively. D. ln-ln-transformed regression of biomass-weighted proportional feeding rate means vs. original mixing model result means; (slope $=0.19$, and $\mathrm{R}^{2}=0.46$ ). Hatched red lines represent the $90 \%$ confidence interval. E. ln-ln-transformed regression of biomass-weighted proportional feeding rate means vs. weighted model result means; slope $=0.22$, $\mathrm{R}^{2}=0.56$. Hatched red lines represent the $90 \%$ confidence interval. doi:10.1371/journal.pone.0022015.g003 . . . . . . . . . . . .

5.1 Species accumulation curve $( \pm \mathrm{SD})$ of the prey observed in the diet of Haustrum scobina at Tauranga Head, constructed using feeding survey observations as the unit of sampling . . . . . . . . . . . 162 
5.2 An analysis of the New Zealand system excluding the prey Patelloida corticata $(P c)$. As expected, there are slight differences in original MixSIR results, however, applying prey availability data to the overlapping prey $M g$ and $X p$ results in a similar increase in the accuracy of prey contribution estimations. A. ln-ln-transformed regression of biomass-weighted proportional feeding rate means vs. original mixing model result means; slope $=0.08, \mathrm{R}^{2}=0.42$. B. ln-ln-transformed regression of biomassweighted proportional feeding rate means vs. weighted model result means; slope $=0.11, \mathrm{R}^{2}=0.53$. The hatched red lines represent the $90 \%$ confidence interval. . . . . . . . . . . . . . .

5.3 Manipulation of the isotopic overlap of prey. A. A mixing space with 2 non-overlapping prey (green, red), two overlapping prey (blue, orange), and a single consumer (black). Here, the overlap of blue and orange prey $\left(w_{\text {blue, orange }}=1\right)$. B) MixSIR estimates of $\%$ contribution to diet for each prey associated with Fig. S5.3A. Green and red are predicted to contribute equally to the consumer's diet, as are blue and orange. C. An alternative mixing space such $w_{\text {blue, orange }}=0$. Here, the blue and orange prey have been symmetrically moved from their previous isotopic values $\left(\delta^{13} \mathrm{C}=28, \delta^{15} \mathrm{~N}=5\right)$ to Blue: $\delta^{13} \mathrm{C}=28, \delta^{15} \mathrm{~N}=4$; Orange: $\delta^{13} \mathrm{C}=28, \delta^{15} \mathrm{~N}=6$. D. MixSIR estimates of $\%$ contribution to diet for each prey associated with Fig. S5.3C. Although the overlap of blue and orange has been manipulated, the geometry of the mixing space retains similar estimates of $\%$ contribution to diet for all prey, with only slight differences in variance. . . . . . . . . . . . . . . . . . . . 164 


\section{List of Tables}

2.1 Mean and standard deviations (SDs) of isotopic values for African predators and prey that were used to assess link-strengths with the Bayesian isotope mixing model MixSIR. See Appendix 2.1 for details. . . . . . . .

2.2 To determine the rate at which estimation of the mean and standard deviation (SD) of the NLID could be estimated as sampling effort increased, exponential curves $\left(y=a e^{b x}\right)$ were fitted to binned numerical results, where each bin represents an increase in ecosystem size by 100 links. Mean error and SD error were calculated as the difference between the true NLID mean and SD, respectively, and that estimated from subsampling PIDs. . . . . . . . . . . . . . . . . . .

2.3 Welch t-test results for $\Delta \mathcal{N}$ and $\Delta \mathcal{M}$ between Models 1 and 2. Model 1 does not include body size constraints, while Model 2 does include body size constraints (refer to Fig. 6, main text). For the most part, the mean values of Model 1 and 2 are not significantly different, with some exceptions indicated by the asterisk $(*) \ldots \ldots . \ldots . . . . . .$.

4.1 Parameters, interpretations, and values in the dynamic state variable model. Parenthetical values (except for $\boldsymbol{\rho}$ ) refer to those for foods: (fruit, grass leaves, USOs, arthropods). Values for $E$ and $R$ are those when no mechanical advantage is included. See methods for relevant references.

5.1 Haustrum scobina prey-specific foraging metrics. . . . . . . . . . . . . . 157

5.2 Mean and standard deviation of species-specific isotopic signatures at Tauranga Head, New Zealand. . . . . . . . . . . . . . . . . . . . . 158 


\begin{abstract}
The structure of mammalian food-webs: interpreting, predicting, and informing estimates of species interactions in paleontological and modern communities by Justin D. Yeakel
\end{abstract}

Patterns of species interactions are both the cause and consequence of ecosystem dynamics. Understanding the origin and function of specific interaction patterns at the ecosystem scale has been a long-term goal in ecology. These efforts are often limited by the enormous size of biological systems, the temporal transience of ecological interactions, difficulties in obtaining reliable measurements [3], knowing what is important to measure in the first place, and the time-scale over which observations are made. In the following chapters, I first introduce a probabilistic framework to incorporate fieldmeasured (rather than experimental) strengths of interactions between species using stable isotope data. This framework provides a means to examine whether different variance structures are predictive of specific interaction patterns, such as nestedness and modularity. Secondly, to assess the impact of global climatic perturbations on mammalian communities over long time scales, I use stable isotope ratios of predators and prey to examine six independent paleontological communities ranging from Europe to Beringia and spanning the Last Glacial Maximum. Both the temporal and spatial evolution of species-specific relationships, as well as community-scale structures, are investigated to understand how changes in climate and prey abundance [99] influenced 
trophic interactions in the late Pleistocene. Although Chapters 1 and 2 concern largescale emergent properties of food-webs, I introduce in Chapter 3 a process-based model designed to investigate the effects of mechanical constraints on the foraging strategies of anthropoid primates, and early hominins in particular. Although this model serves primarily as a predictive tool, towards the end of Chapter 3 I discuss how it can be used instead to inform independent estimates of diet, which may be particularly useful in a paleontological context where data are limited. Finally, in Chapter 4, I extend upon this reasoning and introduce a method by which resource availability data and mixing space geometry can be used to update estimates of trophic interactions from Bayesian isotope mixing models, and demonstrate its utility using data from a New Zealand intertidal community. The four chapters presented here introduce techniques and frameworks by which stable isotopes, statistical mechanics of networks, and process-based models can be used to investigate both the formation and time evolution of the patterns of species interactions in ecological communities. 
To my wife,

\section{Charlene Chow,}

a continual source of inspiration

who does not hesitate to remind me

of the important things,

and my parents,

Doug and Cece Yeakel,

who have always walked the walk.

xix 


\section{Acknowledgments}

I owe a debt of gratitude to a number of people who inspired me and helped me through the process of writing this thesis. ${ }^{1}$ Among the first to introduce me to the scientific process were Seth Newsome, Kena Fox-Dobbs, Brooke Crowley, Katie Snell, Sora Kim, and Patrick Wheatley, and they showed me that answers to questions were within reach, and that the process of answering them could be both challenging and fun. A number of EEB graduate students, in particular the 2007 cohort, have been a continual source of inspiration, and with whom I have enjoyed many scientific 'jam sessions'. Paulo Guimarães Jr., Mark Novak, Ole Shelton, and Erin Vogel were always willing to share their time, troubleshoot, and challenge my thinking about how specific questions could be addressed. I owe an extra debt of gratitude to Paulo Guimarães Jr., who later became a member of my thesis committee, and who introduced me to complex networks. Paulo was always encouraging, willing to meet with little notice, and convinced me quantitative methods were not beyond my grasp (and was sometimes right). I must also thank Nate Dominy, who was a new faculty member in the Department of Anthropology when we met, and over the course of 3 field seasons in Africa - which included flying into the Congo without money, getting clamped in Uganda, and chasing down suspected sheep thieves in South Africa - we proceeded to collaborate on numerous research projects,

\footnotetext{
${ }^{1}$ The text of this dissertation includes a reprint of the following previously published material: Yeakel, J. D., Novak, M., Guimarães, P. R., Jr, Dominy, N. J., Koch, P. L., Ward, E. J., Moore, J. W., et al. (2011). Merging Resource Availability with Isotope Mixing Models: The Role of Neutral Interaction Assumptions. (S. Thrush, Ed.)PLoS ONE, 6(7), e22015. The co-authors listed in this publication directed and supervised the research which forms the basis for the dissertation. Conceived and designed the experiments: JDY MN JWM EJW BXS. Performed the experiments: JDY MN PRG. Analyzed the data: JDY MN PRG NJD PLK. Contributed reagents/materials/analysis tools: MN. Wrote the paper: JDY MN PRG NJD PLK.
} 
some of which are still on-going. And I sincerely thank my advisor troika: Paul Koch, Marc Mangel, and Jim Estes, without whom any of this would be possible. Paul Koch has been an incredible friend and mentor, employing me as a research technician in 2004, and providing an academic home away from home. Over the years, he has encouraged me to seek out the problems that I found most exciting, but always ensured that I was anchored to reality. Marc Mangel introduced me to the wonderful and challenging world of theoretical biology, and his energy and excitement catalyzed the theoretical components of the research presented here. I can't thank Marc enough for facilitating the creative excitement that pushed me to explore concepts that I found both technically challenging and beautiful. Jim Estes provided a strong link to the biology that is at the heart of both the theoretical and empirical components of my thesis work. Jim has always served as a balance, and ensured that the major questions that I was interested in addressing were both carefully formulated and grounded in an understanding of the underlying biology.

Most of all, I thank my family - my parents Doug Yeakel and Cece Yeakel, my sisters Beth Camann and Jenna Yeakel. My parents and my sisters have long been inspirations, and they instilled in me a passion for both art, science, and a general curiosity for the world around us. If I can be half as amazing as I know they are, I will be lucky. And I thank my beautiful wife, Charlene Chow, for enriching my life over the past 8 years. I couldn't have done any of this without your love and support. 


\section{Chapter 1}

\section{Introduction}

...the theories of population interaction are the statistics, or a part of them, in the form of surmises and empirical laws, without the mechanics.

-E. H. Kerner (Bull. Math. Biophysics, 1957)

In August 2003, overgrown trees in a rural Ohio neighborhood caused sagging electrical lines to 'flashover', resulting in a dangerously spiked increase in electrical current. To protect the power grid, automatic relays quickly disconnected the faulty line, requiring neighboring lines to handle additional load. Because excessive use of air conditioning units had critically stressed the surrounding grid, the redirected current was great enough to trip protective relays in neighboring lines. Eventually, this sequence cascaded across northeastern North America, resulting in the shutdown of 100 power plants ranging from Northern Ontario to Boston, Massachusetts, leaving 55 million people without power and contributing to 11 fatalities.

The power grid is an example of a complex network, where numerous electrical relays (the nodes of the network) are connected by transmission lines (the links of the 
network) in a specific topological arrangement. Analogously, communities of interacting animals can be described as a network, where the nodes of the system represent species and links represent interactions between species. As current flows through transmission lines from one relay to another, the behavior of one species can affect another species, though often in a more complex and less direct way. The power grid and a community of interacting animals are very different networks, but are similar in that the structure of interactions can have large effects on their respective dynamics. As the distribution of current flowing through transmission lines resulted in a cascading failure of the power grid, the structure and strengths of interactions between species may have similarly large effects on the dynamics of ecosystems.

In biological communities, the impact of structure on dynamics is most easily observed on smaller scales. First consider an interacting predator and its prey (Fig. 1.1A): in some cases after an initial transient period of interaction (where the abundances of both species may fluctuate out of sync), the rate of predator and prey growth will meet at a steady state where the abundances of both populations no longer change. These predator-prey dynamics can be altered as the structure of the network changes. For instance, if a single predator consumes two prey species (Fig. 1.1B), the predator can switch between prey as the abundance of one changes relative to the abundance of the other. This process, resulting in asynchronous oscillations of the two prey as they alternatively become victims to the predator, is known as apparent competition because the prey populations appear as if they are competing for a limiting resource [71]. Although asynchronous oscillations can be observed in some cases, differences between the 
prey, or constraints limiting the foraging habits of the predator may bias predation of certain prey in natural communities.

The structural organization of species interactions is known (both theoretically and empirically) to influence the dynamics of relatively smaller-scale systems [106, 183], and this connection has been extended to large communities with hundreds of species and thousands of interactions $[55,140,171]$. When such systems are composed of consumers and their resources, they are called food-webs. The organization of foodwebs can be summarized by the statistical properties of their networks. For example, nestedness describes the degree to which consumers have food resources that overlap with other consumers [90], while modularity describes the degree to which consumers have diets that are separate from other consumers [91]. There are theoretical arguments that show a strong connection between certain statistical properties of food-webs, such as nestedness and modularity, and dynamics [184, 179].

The cause of the 2003 power failure was both instigated and amplified by the magnitude of current being redirected through neighboring power lines. Similarly, the magnitude of trophic interactions in biological food-webs can have a large impact on ecosystem dynamics, and as with structure, dynamical implications are more easily observed on smaller scales. Trophic cascades occur when a perturbation to a species' abundance at one trophic level has a cascading influence on the abundances of species many trophic levels away. For instance, the presence or absence of cougars can drastically alter the abundance of butterflies, due to the impact that cougars have on deer, the corresponding impact that deer have on the plants they consume, and finally the re- 
lationship between these plants, their competitors, and butterflies [154]. Such cascades depend primarily on the organization of interactions with different magnitudes within the food-web (for an example, see Fig. 1.1C).

Traditionally, the strength of an interaction in a food-web is defined by an observed change in abundance of one species with respect to the removal of another [18]. These measurements are quantified from manipulation experiments, where the removal of species is performed in a controlled setting (thereby limiting the size of the community being examined), and where the effects of both direct (e.g. predation) and indirect (e.g. competition) interactions are measured. However, most ecosystems cannot be experimentally manipulated and some may not be observable. As a consequence, measures of food-web structure (particularly for large systems) typically ignore interaction strengths [141] and instead analyze the structure of the binary system, where only link presence/absence is quantified. Ignoring the effects of interaction strengths on structure likely biases our understanding of how structure contributes to ecological dynamics.

An obvious alternative to the experimental measure of interaction strength is the flow of biomass from one species to another - here and hereafter distinguished as link-strength. In a food-web network, link-strengths can be defined as the proportional contribution of resources to the diet of a consumer (cf. [14]). Such a measure necessarily represents a consumer-centric component of trophic interactions, although prey-centric measures can sometimes be subsequently derived. Importantly, the proportional contribution of resources to consumer diets can be estimated from stable isotope data using Bayesian isotope mixing models $[122,139,127]$. Using these methods, quantification of 
link-strengths is extendable to paleontological communities, vastly extending the temporal range over which food-webs can be examined and compared.

How does the inclusion of link-strengths change our understanding of foodweb structure? Can link-strengths be used to compare food-webs in non-experimental settings, and can isotopic techniques be used to explore how mammalian community structure has changed over large timescales? Moreover, at the scale of an individual forager, can specific anatomical limitations that preclude feeding on certain foods be used to predict diet, thereby establishing constraints that limit how species become linked to the larger food-web? In the next four chapters (2-5), I use ratios of stable isotopes to quantify link-strengths between interacting species in addition to mathematical modeling to both compare and predict the structural organization of mammalian predator-prey networks as well as the forces that constrain species interactions over continental spatial-scales and temporal-scales that span the Plio-Pleistocene.

In Chapter 2, I show how link-strengths can be estimated directly from stable isotope ratios using Bayesian isotope mixing models, and how these estimates of link-strengths can be integrated into food-web networks. Measuring the organization of species interactions with respect to link-strength magnitude can reveal whether different structures are associated with different magnitudes of link-strengths in a community. Because dynamics depend on both structure and the magnitudes of link-strengths, this added detail is vital for connecting structural features of food-webs to potential dynamics. As an example, we show that the strongest link-strengths in two African predatorprey networks are highly modular relative to a Canadian boreal forest predator-prey 
network, meaning that mammalian carnivores in Africa typically have diets that do not overlap, but only for prey that contribute strongly to predator diets. In contrast, African predator-prey networks appear nested when link-strength magnitudes are not considered. It is likely that both the nested structure of the unweighted system (where link-strengths are not considered) and the modular structure of the strongly connected species strongly affect dynamics.

Predicting the response of mammalian communities to large-scale changes in climate and/or habitat has important conservation implications, however the timescale over which mammalian food-webs operate is large, limiting the usefulness of studying only modern communities. As perturbing vital resource networks such as the power grid is generally discouraged, large ecosystems are beyond the reach of manipulation experiments; however as with the power grid we can use past perturbations to examine their impact of food-web structure. In Chapter 3, I investigate the structure of six mammalian predator-prey networks quantified from stable isotope data. These communities ranged from Europe to Beringia and spanned the Last Glacial Maximum (LGM), a global climatic perturbation. I show that certain taxonomic groups had constrained dietary strategies across Eurasia, and many were affected by increasing caribou abundance [99] across the LGM. Moreover, I show that European and Beringian communities had strongly divergent structural properties despite strong species contiguity, and suggest a biogeographic mechanism for these differences.

Extending further into the past and towards the decision-making strategies of individuals, I next explore to what extent Plio-Pleistocene hominin foraging behavior is 
limited by the physiological constraints imposed by their teeth. I present a process-based stochastic dynamic program to quantify the impact of enamel wear on foraging decisions, and the potential fitness benefits introduced by altering food mechanical properties. Because model results are discussed in terms of paleo-dietary data (e.g. the isotopic ratios of hominins), our framework can be used to generate Bayesian priors that quantify predicted diets. Accordingly, these process-based models can be used not only to predict but inform independent estimates of paleo-diet in a Bayesian framework.

Finally, extending upon prior construction from process-based models, I present in Chapter 5 a means by which independent prey availability data can be used to update estimates of dietary contribution quantified from stable isotope mixing models. Isotope mixing models calculate the proportional contribution that prey resources contribute to predator diets as a function of both prey and predator isotope ratios. Thus, estimation accuracy is contingent on the 'mixing space', or orientation of prey and predator isotope values relative to each other. If multiple prey have similar isotopic values, their estimated contribution will be similar, despite potential differences in availability. Prey availability data, including but not limited to differences in abundance or predator preference, would impact predator diet composition, and I introduce a technique by which this independent information can be used to update mixing model predictions. 


\section{$1.1 \quad$ Figures}

A.

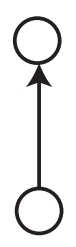

C.

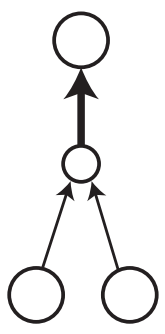

B.

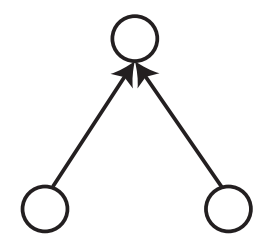

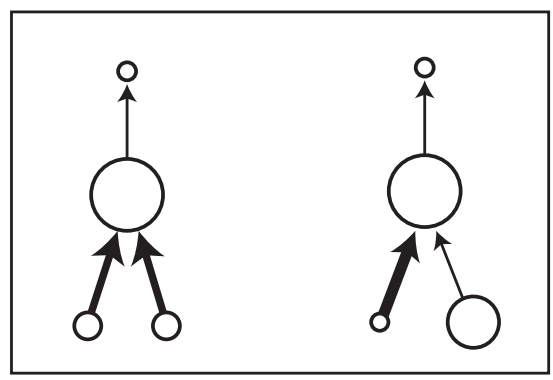

Figure 1.1: A. The predation of a prey by a predator. The arrow represents the flow of biomass such that the top node denotes the predator and the lower node represents the prey. B. The predation of two prey by a single predator. If the prey are preferred similarly by the predator, this structure results in asynchronous oscillations of prey abundance (apparent competition). See panel C. for a scenario where one prey is preferred over another. C. A tri-trophic system, where a top-predator consumes a meso-predator, which in turn consumes two prey. The sizes of the nodes denote relative abundance, and the thickness of the arrows denote the magnitude of the biomass flow. The top-predator in the leftmost network is relatively abundant, depressing the meso-predator population, thereby limiting the meso-predator's effect on the two prey populations. The two rightmost networks (boxed) have reduced top-predator populations. In both cases, the meso-predator has greater abundance due to decreased predation pressure. However, in one scenario, both prey are preferred equally, resulting in apparent competition dynamics. In the second scenario, one prey is preferred over another, resulting in an asymmetric cascade. Such preferences could result from specific anatomical constraints of the meso-predator, a scenario explored in Chapter 4. 


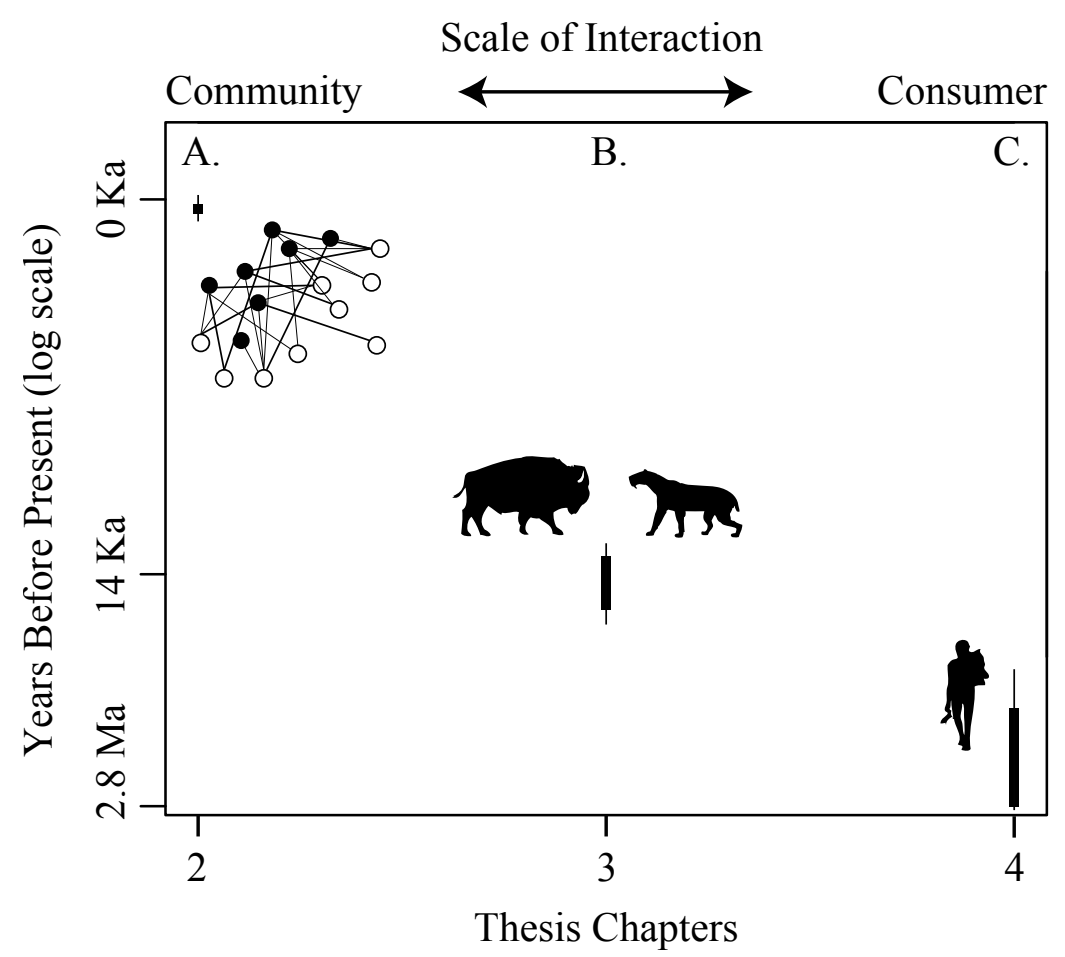

Figure 1.2: Distribution of Thesis Chapters as a function of the temporal range and the scale at which interactions are considered. Chapter 4 is not included because it is a methodological report, and is applicable to any time period and multiple scales of interaction. From left to right, silhouettes represent a food-web network, the Pleistocene species Bison bison and Homotherium serum, and the Plio-Pleistocene hominin Paranthropus robustus. 


\section{Chapter 2}

\section{Probabilistic patterns of interaction:}

\section{The effects of link-strength variance on}

\section{food-web structure}

J D Yeakel, P R Guimarães Jr., M Novak, K Fox-Dobbs, P L Koch 


\section{Abstract}

Patterns of species interactions affect the dynamics of food-webs. We examine how variation in strengths of interactions can be described hierarchically, and how this variation impacts the structure of species interactions in food-webs. Using three mammalian (two African and one Canadian) food-webs quantified from stable isotope data, we show that the distribution of variation in strengths among interactions can be estimated from a limited number of observations. Moreover, this distribution informs food-web structure, especially the key role of dietary specialization. Our results show that exclusion of link-strength variability results in biased estimates of nestedness and modularity within food-webs, while the inclusion of body size constraints only marginally increases predictive accuracy. We find that modular structures are the consequence of strong link-strength in both African systems, while nested structures are not significantly present in any of the three food-webs.

\section{$2.1 \quad$ Introduction}

Ecosystem dynamics are strongly sensitive to the structural organization, or topology, of trophic interactions among species. Food-webs are often characterized by 'qualitative networks', where only the presence/absence and direction of interactions (links) are established among species (nodes) [54]. Quantitative networks include linkstrengths, which establish a relative importance to each link (Fig. 2.1A). Link-strengths are starting to be considered in food-webs [141], and both empirical and theoretical 
work suggests that variance in strength among links in a network has a large impact on structure, and by extension, dynamics $[12,132]$. Because species interactions vary over space and time, and are estimated imprecisely, the strengths of individual links are probabilistic (where a given link-strength has an associated probability; Fig. 2.1B), however it is not clear how to integrate this information into ecological networks, or how such variation impacts structure. This is a key question, because the structure of species interactions ultimately affects ecosystem dynamics [5, 178].

Specific patterns of interaction are often observed in ecological networks [14, 184, 90]. Some of these structures have important dynamical implications and/or may contain information regarding the role of biological factors, such as body size, in shaping the organization of ecological communities [202]. Body size constraints are often realized by the inability of predators to consume prey due to differences in their respective body sizes $[202,166,148]$. Two potentially important structural features of food-web networks are nestedness, which arises when predators have incompletely overlapping diets, and modularity (compartmentalization), where higher densities of links exist between certain predator-prey groups [118, 184].

Nestedness is often observed in mutualistic networks [13] and some food-webs [90], and may occur when organisms follow hierarchical rules of interaction [148], including those generated by allometric predation limitations (body size constraints) [166]. Theoretical food-web models that assign links hierarchically and that allow some prey overlap among predators reproduce many statistical features of empirical food-webs [180]. Recent theoretical work has shown nested interactions [184], as well as interac- 
tion patterns predicted by food-web models [6], to be unstable in antagonistic networks. In contrast, modularity may stabilize food-webs $[113,184]$ by minimizing extinction cascades [179]. However, analysis of the incidence and impact of these structural properties have been confined to qualitative food-webs. As such, the presence of nestedness and modularity, as a function of link-strength, has not been assessed. This is of key importance because weak and strong links may differently affect species persistence after a perturbation $[115,89]$.

Although link-strength variability adds substantial complexity to the description of food-webs, it is necessary to understand if and how variation in link-strengths impacts food-web structure. Furthermore, quantification of this variability may enable predictions of food-web topological properties. Here we show that link-strength variability can be assessed as a hierarchy of distributions, such that ecological variation and measurement uncertainty are quantified on multiple scales within a food-web, and that these distributions can be estimated from incomplete data. We then use three empirical food-webs quantified from stable isotope data to assess 1) if link-strength distributions predict food-web structure, and 2) the effect of body size constraints on food-web structure. Our results show that even limited knowledge of the variance in strength among links provides information regarding the topological properties of three empirical food-webs, and that structural organization changes as a function of link-strength across food-webs. In addition, although body size limitations (defined as above) constrain species interactions, our results indicate that the inclusion of such constraints only marginally improves predictions of food-web structure. 


\subsection{Methods}

Links that denote interactions between species within food-web networks can be described qualitatively (i.e. by the presence or absence of links) and quantitatively (i.e. by links that are weighted with single values typically normalized to range between 0 and 1) (Fig. 2.1A). Here we expand the quantitative approach to describe links probabilistically, such that link-strengths for a given pairwise interaction have associated probabilities. We define link-strength in terms of the proportional biomass flow from prey to a consumer [14]. Thus, links connecting a single consumer to its prey denote the proportional contribution of biomass of prey to a predator and sum to one (Fig. 2.1B). Furthermore, link-strength probability distributions are described hierarchically, such that variance among links and variance within links are considered separately.

\subsubsection{Strength variance in food-web networks}

Quantitative food-webs have been shown to have long-tailed link-strengths distributions, with many weak interactions and few strong interactions [203, 14, 188]. This distribution describes the variation of strength among links in a system (hereafter Network-Level Interaction Distribution, NLID; Fig. 2.1C). The long-tailed nature of this distribution is due to the multiplicative analogue of the central limit theorem [47]. The shape, or parameterization, of these distributions can have a significant impact on the stability of theoretical ecosystem models $[89,75]$, though its influence on topology is not

well understood. An NLID can be constructed for both quantitative and probabilistic 
food-webs (see below).

Link-strengths for any observed food-web are estimates of the interaction patterns among species within a community, and therefore all food-webs are inherently probabilistic. The variation of strength within links in a food-web reflects the temporal and spatial variation in prey consumption, dietary variation among individuals, and measurement uncertainty. Within-link variance is typically only considered in theoretical investigations or in controlled laboratory settings. We distinguish such distributions by referring to them as Pairwise Interaction Distributions (PIDs; Fig. 2.1D), which are unique for each link in the system. We note that the NLID and the set of PIDs describing links in a food-web are hierarchically related, such that the mean and variance of the NLID is ultimately determined by the parameterization of all PIDs in a system.

\subsubsection{Quantifying link-strength and variance in empirical systems}

We quantified the PIDs for three mammalian food-webs using stable isotope data. Ratios of stable isotopes (typically of carbon and nitrogen) can be used to infer trophic relationships between consumers and prey $[84,128]$. The carbon and nitrogen isotope values of a consumer, with respect to potential prey, characterize the isotopic niche space of the consumer $[128,122]$. It has been shown that the isotopic niche is comparable to the traditional concept of a dietary niche [128]. Posterior probability distributions of the proportional contributions of each prey to a consumer's diet can be numerically estimated with Bayesian isotope mixing models [122], and represent the PIDs of the food-web. 
These proportional contribution estimates incorporate the uncertainty inherent to isotopic approaches including analytical error, isotopic variability in prey and consumers, poorly constrained fractionations between trophic positions [29], isotopic similarity of multiple prey [206], and underdetermined or non-unique solutions [122]. PIDs calculated from a mixing model are each marginal distributions of a single large multivariate (Dirichlet) distribution. As such, each marginal PID is constrained such that the link-strengths connecting a given consumer to all of its prey must sum to one.

Posterior distributions of Bayesian isotope mixing models are challenging to determine analytically, but can be estimated numerically using importance sampling approaches [122]. Numerical approximations of the PIDs and NLID of a food-web can be assembled directly from mixing model output. A set of potential contribution-to-diet values are calculated, where each element of the set represents a 'potential' combination of link-strengths between the consumer $j$ and its $n$ potential prey and sums to one. For our study, we generated 1000 such combinations, and organized them as rows of a matrix $\mathbf{W}_{j}$, where each column represents different potential contributions of prey $k$ (out of $n$ available prey). A PID for a given consumer $j$ linked to a prey resource $k$ is then described by a vector of 1000 potential 'prey contribution values', $\mathbf{w}_{j k}$, with each prey $k$, represented by a column in the matrix $\mathbf{W}_{j}$ (see Fig. S2.1 for additional details). Overall, a set of randomly drawn rows for each matrix $\mathbf{W}_{j}$, across $m$ consumers in the system, represents a set of link-strengths connecting every consumer and resource in the food-web, and is equivalent to a quantitative network, where each link has a single link-strength. By extension, the full set of matrices $\mathbf{W}_{j}$ for $j=1, \ldots, m$ for each of 
$m$ consumers in the system, describes the probabilistic network, or alternatively, an ensemble of potential quantitative networks. The NLID for the probabilistic network can be assembled by sampling evenly across all PIDs $\left(\mathbf{w}_{j k}\right)$ for each matrix $\left(\mathbf{W}_{j}\right)$. To evaluate the structural properties of the probabilistic food-webs, we considered each potential quantitative food-web in the ensemble independently, such that a range of structural values were calculated.

To quantify structural properties as a function of link-strength, we developed a cutoff algorithm to sequentially measure structural metrics as links with low contribution-to-diet values are successively 'trimmed' from the food-web [187]. This procedure was carried out for every potential (1000) quantitative food-webs in the probabilistic ensemble. This cut-off algorithm iteratively eliminates the weakest links in a system, allowing us to explore the structure of both the entire and just the core resources used by consumers. Given a potential quantitative network within an ensemble, we binarize the link-strengths [10]. For the cutoff value $i$, a link exists if and only if it has a link-strength $>i$. The structural properties of each qualitative network were then analyzed for a set of cutoff values $i$ (i.e. $i=\{0.1,0.2, \ldots, 0.5\}$ ). For each measured network property, this algorithm quantifies five values (one for each cutoff), describing how structural properties change as weak links are successively eliminated.

We examine three mammalian food-webs with two trophic-levels using stable isotopes to quantify link-strength distributions (see Appendix 2.1 for details). One of these isotopic datasets is from a mammalian community in Saskatchewan, Canada (9 predators and 8 prey, Fig. S2.1), an inland boreal forest ecosystem interspersed with 
isolated rivers and wetlands [192]. Two others are from East Africa: Lake Naivasha (3 predators and 8 prey, Fig. S2.3) [9], and Amboseli (4 predators and 10 prey, Fig. S2.4) [87], both savanna-woodland mosaics spanning high altitude montane forests to low altitude grassland-dominated environments across southern Kenya. By restricting our analysis to the mammalian components of these food-webs, we can more accurately quantify mass flow between species, though we acknowledge that these are necessarily subsets of larger communities. This approach is contrasted with the common alternative to construct a food-web with more species, but with less descriptive detail. Furthermore, we limit our analysis of each community to locally abundant species $>4 \mathrm{~kg}$, thereby focusing on interactions among larger consumers and their potential prey. Because of the energetic restrictions of large consumers, these food-webs are considered to be fairly isolated from (or connected by weaker interactions to) other animals in the ecosystem. Forbidden interactions (where the strengths of certain links have an a priori value of ' 0 ') were built into mixing models for each empirical system based on body size differences between consumers and prey, following observations by [166], and detailed in Figs. S2S4. Pairwise interaction distributions for these systems were quantified with the mixing model MixSIR [122].

\subsection{Analysis and Discussion}

In trophic systems, the topology of species interactions dictates the extent to which consumer species share their resources. We tested if networks generated by ran- 
domly sampling the NLID reproduce the patterns of interaction observed in empirical food-webs. We assessed two components of probabilistic food-web structure by analyzing a series of potential quantitative food-webs: 1) by the topology of link-strengths connecting individual species or 2) by the general topology of link-strengths, irrespective of species (Fig. 2). Model systems drawn randomly from NLID may retain the weighted structure of the original food-web but have alternative arrangements of link-strengths among species (Fig. 2B), or they may have different weighted structures as well as an alternative arrangement of link-strengths among species (Fig. 2C). Thus, deviations between empirical and random food-webs may indicate the role of underlying ecological factors in shaping patterns of interaction in food webs. For example, a weak interaction between a predator and a prey may exist due to a strong interaction between the same prey and a different predator, leading to niche partitioning. However, networks drawn randomly from the NLID will not conserve this pattern. These NLID-derived networks provide a benchmark to study the role of ecological factors other than those generating the distribution of link-strengths in shaping food-webs. It is first necessary to estimate the NLID shape; although the true' NLID cannot be known (see below), we ask to what extent it can be estimated from PIDs for food-webs where the majority of links are unknown and unobserved. If the NLID can be estimated when data are limited, it is then worthwhile to explore whether and to what extent it can predict structure in empirical food-webs. 


\subsubsection{Estimating the NLID from limited observations}

Most ecosystems are difficult to observe and have an unknown number of interacting species. While the true NLID of a food-web may be unknowable, we devised a model to determine the accuracy of NLID estimation by sub-sampling observed interactions. The distribution of strengths among links (the true NLID) was assembled from a series of PIDs that were generated from random independent beta distributions (which incorporate the constraint that link-strengths vary between 0 and 1 ;

$\alpha \in\{0.01: 10\} ; \beta \in\{0.01: 100\}$ ), for a continuum of systems varying in size from 2 to 500 links. NLID assembly was then carried out, as described above for numerically generated posterior probability distributions, for link-strength vectors $\left(\mathbf{w}_{j k}\right)$ randomly drawn from each beta distribution.

To estimate the accuracy by which sub-samples of link-strengths from a foodweb of a given size can accurately estimate the true NLID, we 1) randomly sampled from the available PIDs, 2) constructed an estimated NLID, and 3) measured the absolute difference of the mean (mean error) and standard deviation (SD error) of the true and estimated NLIDs to assess the similarity between the two distributions. For foodwebs with $100,200, \ldots, 500$ links, modeled results were fit to an exponential function to determine the rate at which the observed mean and standard deviation of the estimated NLID converged to the known moments of the true NLID as sampling effort increased (Fig. 2.3; Table S2.2).

In a weighted or probabilistic food-web, the shape of the NLID may constrain 
network structure and impact potential dynamics. Our theoretical sampling experiment revealed that the error of NLID estimates decreased exponentially as the proportion of sampled PIDs increased (Fig. 2.3; Table S2.2). Thus, sampling a small proportion of PIDs will yield accurate estimates of the NLID, particularly for large food-webs. The exponential nature of this relationship indicates that, even for smaller systems, a relatively modest sampling effort will yield fairly accurate estimates of the real NLID. Because the shape of the NLID can be estimated when the food-web is incompletely sampled, it is worthwhile to explore whether the NLID contains information regarding the topological organization of link-strengths.

\subsubsection{Assessing structure from the NLID}

Several structural properties were quantified for each of the three empirical food-webs. Primary among these were nestedness $(\mathcal{N} ;$ Fig. 2.4A) and modularity $(\mathcal{M}$; Fig. 2.4B), because these properties are thought to have important ramifications for the dynamics of ecological systems $[184,90,179]$. To quantify nestedness, we use the NODF (Nestedness based on Overlap and Decreased Fill) metric [7], where a value of 0 denotes an un-nested network and a value of 1 represents a fully nested network.

Modularity has traditionally been quantified as the local density of links in a

network [38], although other metrics exist [66]. Because our food-webs have two trophic levels, we use the density of squares to estimate the average local link density in a network [211]. A 'square' occurs when four nodes are connected by at least four links, where the average density of squares $\left(\bar{\rho}_{s}\right)$ equals the number of squares in a network 
divided by the number of potential squares in a completely connected network, i.e. the local link density. This metric has the advantage of being analytically tractable for any network size, whereas other metrics use optimization methods [66]. As the connectance, $C$, of a network increases $(C=l / m n$, where $l$ is the number of links in a network, and $m$ and $n$ are the number of prey and predator species, respectively), the local link density increases. We calculated modularity as

$$
\mathcal{M}=\frac{\bar{\rho}_{s}-C}{\bar{\rho}_{s}+C}
$$

If the average local link density becomes greater than the overall link density, $\mathcal{M} \rightarrow+1$; if the average local link density becomes less than the overall link density, $\mathcal{M} \rightarrow-1$ $[10]$.

In addition to nestedness and modularity, we also quantified: 1) The frequency of networks characterized by low local link density $(\mathcal{M}=-1$; Fig. 2.4B); 2) the number of isolated components in a network, defined by disconnected subgraphs, each with $\geq 2$ connected species (Fig. 2.4C); 3) the number of isolated species in a network (Fig. 2.4C); and 4) the frequencies of four-species subgraphs (subunits of 2 predators connecting 2 prey) of which there are seven possible arrangements (Fig. 2.4D). The quantification of these six structural features permit a holistic description of interaction patterns within food-webs that, as a whole, can be used to compare the structural similarity of independent food-web networks.

We assessed the ability of food-web models derived from NLIDs, with identical forbidden link structures as the empirical systems, to predict the six structural prop- 
erties of empirical food-webs. Predictive ability was scored by a similarity index $\left(s_{i}\right)$ which varies between 1 (absolute similarity) and 0 (absolute dissimilarity) across cutoff values $i$ (see Appendix 2.2 for details). We tested whether a good empirical parameterization of the NLID was enough to predict the structural properties of empirical food webs. If so, then detailed quantification of strength variation within-links (PIDs) would not be necessary to characterize network structure. In our models we varied the NLID standard deviation (SD) towards higher and lower values than that measured from the empirical food-web, altering NLID shape. We then measured the structural similarity of empirical food-webs against models drawn from the NLID with altered SDs. If the NLID is strongly predictive of the empirical food-web structure, we expected a maximum value of $s_{i}$ averaged across cutoffs $i\left(\bar{s}_{\max }\right)$ when the model food-webs are drawn from the NLID matching that of the empirical food-web (Fig. 2.5A-C). This would suggest that the NLID encodes some structural properties of the empirical system. By extension, it would indicate that the structure of empirical food-webs is - in part - as would be predicted if species interactions were not structured by ecological tradeoffs.

As the shape of the model NLID is altered by changing its SD relative to the empirical SD, the effects on the underlaying patterns of interaction are not straightforward. To understand how structural variation affects these patterns, we explored how dietary unevenness varies as the shape of the NLID is altered. Dietary unevenness describes consumers connected by strong link-strengths to a small subset of available prey, and dietary evenness describes consumers connected by weaker link-strengths to a greater proportion of available prey; see Appendix 2.3 for details. Dietary unevenness 
can be used to measure the degree of specialization among consumers. However, if prey abundance is strongly skewed, even a predator without prey preferences (a generalist consumer) would look strongly uneven, incorrectly suggesting specialization. As a consequence, dietary unevenness cannot be compared among predators that consume prey with different abundances. Here we compare consumer dietary unevenness across the same set of prey within each site, which is equivalent to dietary specialization among sympatric consumers. We measured the degree of specialization for consumers in empirical and NLID-derived food-webs as the SD of the NLID was varied (Fig. 2.5D-F).

Both Saskatchewan and Amboseli had highest similarity when the standard deviation matched the empirical NLID SD (NLID SD $=0.27$ for both systems; $\bar{s}_{\max }=$ 0.93 and 0.95 for Saskatchewan and Amboseli, respectively; Fig. 2.5A,B). The $\bar{s}_{\max }$ for Lake Naivasha was associated with a higher NLID SD than predicted by the empirical food-web $\left(\mathrm{SD}=0.18, \bar{s}_{\max }=0.96\right)$, however this value is not significantly different from the $\bar{s}$ for the empirical standard deviation $(\mathrm{SD}=0.16, \bar{s}=0.95$; Welch's two sample t-test $(\mathrm{df}=7.9): \mathrm{t}=-0.17, p=0.86$; Fig. $2.5 \mathrm{C})$. The sensitivity analysis revealed that NLID-based food-webs that share the same body-size based forbidden link structure have a high degree of structural similarity to empirical food-webs. Furthermore, we found that similarity is strongly sensitive to the accurate parameterization of the NLID. This pattern holds across most cutoff values, especially when both weak and strong links of the food-web were considered (i.e. low cutoff values) (Fig. 2.5A-C). By contrast, when only strong links (higher cutoff values) were considered, SDs higher than the empirical SD were equally suitable for reproducing structure observed in the empirical food-webs. 
The shape of the NLID had a large impact on the degree of dietary specialization among consumers (Fig. 2.5D-F), though the relationship is nonlinear; low NLID SD leads to model food-webs dominated by generalists, while high SD leads to model food-webs dominated by moderate to strong specialists. Model food-webs matching the SD of the empirical NLID (maximum similarity between the model and empirical foodwebs) resulted in intermediate values. Furthermore, an analysis of specialization among consumers revealed higher values than expected compared to model networks. This underestimate model systems indicates that Saskatchewan and East African predators have stronger prey preferences than expected from model networks. Moreover, saturation of $\bar{s}_{i}$ at high SD for the strongest links in the food-webs $(i=0.4,0.5)$ suggests that structure collapses similarly for empirical and model systems when food-webs are dominated by specialist consumers. This indicates that 1) the inclusion of weaker links in the system has a disproportionate impact on structure, particularly when the food-web is dominated by specialists, and 2) the structures imparted by the strongest links in the food-web are not different from those generated by random processes and are less sensitive to NLID shape. Importantly, our results show that much of the structure of these food-webs is predictable from link-strength variance at the level of the food-web. This means that the variance in strength among links in a food-web, without regard to the specific and unique interactions among species, strongly influences structure. Whether models derived from the NLID accurately predict specific structures such as nestedness or modularity is not known. 


\subsubsection{Body size constraints and ecological implications}

Body size differences between predators and prey affect food-web structure in many systems [202], particularly those in marine and intertidal environments [17].If body size differences between a predator and a given prey are such that predation is too energetically inefficient or risky, predation is unlikely to occur. Here we assume body size constraints lead to forbidden links $[13,4,135]$. To assess the role of these constraints in shaping empirical food-webs, we compared two probabilistic food-web models: one that does not include body size constraints (Model 1), and one that retains the same number and structure of forbidden links as the respective empirical food-web (Model 2). The strengths of non-forbidden links for both model food-webs were randomly drawn from the NLID of the empirical probabilistic network. These models assume that species interactions are independent and the correlations among link-strengths due to ecological factors that affect predator foraging can be ignored. We note that when considering both strong and weak interactions (i.e. cutoff $=0$ ), Model 1 links all predator-prey pairs, while Model 2 shares the same qualitative link structure as the empirical food-web.

Departures in the empirical food-web structure from predictions generated by model networks would indicate that link strengths are constrained by different physical or biological factors. These factors include specialization on alternative prey, competition among consumers leading to low resource overlap, or spatial heterogeneity that affects prey consumption. Because some structural properties, such as nestedness [57], correlate with food-web size [55], we report the relative difference of structural property 
values between empirical and model food-web ensembles. These relative structural metrics are expressed as $\Delta \mathcal{F}_{i}=\mathcal{F}_{i \text {,empirical }}-\mathcal{F}_{i \text {,model }}$, where $\mathcal{F}_{i}$ refers to the value of either nestedness $\left(\mathcal{N}_{i}\right)$ or modularity $\left(\mathcal{M}_{i}\right)$ at cutoff $i$. A value of ' 0 ' indicates no difference between the empirical food-web and corresponding model; if $\Delta \mathcal{F}>0$ the empirical food-web has a higher value than the model; if $\Delta \mathcal{F}<0$ the empirical food-web has a lower value than the model.

The comparison of $\Delta \mathcal{N}_{i}$ and $\Delta \mathcal{M}_{i}$ for each food-web reveals that model networks without or with body size constraints (Models 1 and 2, respectively) lead to similar structural predictions across cutoff values. The model that incorporates body size constraints predicts structures that are generally more consistent with those of empirical food-webs (Table S2.3), showing that the inclusion of body size constraints does increase the predictive accuracy of food-web models. Moreover, our results reveal that $\mathcal{N}$ and $\mathcal{M}$ are strongly inversely related across cutoff values (particularly for Model 2; see Fig. S2.5), a relationship that has been observed in previous studies [57]. Our analyses show that it is also valid across cutoff values within probabilistic food-webs.

The structural analysis of the Saskatchewan system reveals lower than expected nestedness for low cutoff values, and higher than expected nestedness for high cutoff values (Fig. 2.6A,D). This indicates that nestedness is a property of strong links in this system. By contrast, modularity was marginally higher than expected for low cutoff values, and there was strong similarity between empirical and model food-web modularity for high cutoff values. These relationships were maintained when smaller predators were eliminated from the system (such that the species' body size ranges 
were more comparable to those of the African food-webs; Fig. S2.6). Importantly, the structural properties of both African systems were qualitatively similar. Across all cutoff values, Amboseli and Lake Naivasha showed less nestedness than predicted; empirical nestedness values were lower than those predicted by both model food-webs for cutoff values 0.2 to 0.4 (Fig. 2.6B,C). As such, Amboseli and Naivasha are characterized by groups of consumers that show a low degree of overlap in prey use, particularly for strongly-linked prey (Fig. 2.6E,F).

A comparison of $\Delta \mathcal{N}$ and $\Delta \mathcal{M}$ across our empirical food-webs has three important implications. First, random interactions based on the empirical NLID (Models 1 and 2), do not necessarily result in accurate predictions of nestedness and modularity across cutoff values. Moreover, accuracy is lowest for African systems (Fig. 2.6). For both African food-webs, structural differences are generally highest for cutoff values 0.2 to 0.4 , which shows that stronger links are less nested and more modular than predicted. However, when the weakest links are considered, accuracy is generally increased (such that $\Delta \mathcal{N}$ and $\Delta \mathcal{M}$ values are closer to zero). If these systems were investigated without considering link-strengths (where only the weakest interactions would be ignored or not measured), the minimal structural differences (low $\Delta \mathcal{N}$ and $\Delta \mathcal{M}$ values) would imply that models drawn from the NLID are wholly predictive of structure, i.e. that trophic interactions are insensitive to strong correlations between link-strengths. Our analysis of higher cutoff values reveals such a conclusion to be false, highlighting the importance of considering effects of link-strengths when quantifying food-web structure. We hypothesize weak and strong links to differ in the candidate processes generating 
their patterns of interaction, whereas the role of random processes is restricted to the qualitative structure of food-webs.

Second, the strongest link-strengths of the Saskatchewan system are more nested and less modular, while both African systems exhibit lower nestedness and higher modularity than predicted. The similarity of African food-webs relative to Saskatchewan may reflect larger-scale landscape-driven constraints on species interactions. Recent work in the Serengeti has shown the food-web to be compartmentalized with respect to spatial guilds, where local habitat mosaics result in compartments of tightly interacting species [15]. The results of our work lend general support to this finding, and reveal that modularity is emphasized for stronger links in the system. Furthermore, our analysis suggests that systems that are more spatially homogenous (such as the coniferous woodlands of Saskatchewan) may be less modular and not different from systems with randomly-drawn link-strengths. Future work could use our approach to investigate the relevance of spatial organization in structuring trophic interactions across landscapes.

Third, the limited improvement in structural predictions when body size constraints are included (Model 2) suggests that forbidden links do influence link-strength distributions, but not significantly (Fig. 2.6; Table S2.3). The isotopic values of a predator with respect to its prey integrate the effects of prey body size (such that prey with prohibitive body sizes contribute less to a predator's isotope values, even when they are included in the mixing space), regardless of whether forbidden link structures are instituted into an isotope mixing model. Accordingly, the establishment of forbidden links in Model 2 may be redundant when link-strengths are considered and when their distri- 
butions are accurately estimated. Although including forbidden links increased model accuracy, if the distribution of weaker links in a system can be known such that the NLID is accurately parameterized, they likely serve a similar function in quantitative food-webs as does the inclusion of forbidden links in qualitative food-webs.

A detailed analysis of food-webs where both the strength and variance of links can be quantified will enable a more complete understanding of the primary drivers of food-web structure. Importantly, the construction of food-web networks from the flow of biomass given by the isotopic distributions of predators and prey is a framework that permits analysis of systems that are difficult to observe, and is extendable to historical or paleontological communities. In the future, the exploration of food-web structure across space and time will be vital for understanding its origin and function, as well as its sensitivity to environmental or climatic perturbations.

\section{Acknowledgments}

We thank J. Bascompte, D. Costa, N.J. Dominy, J.A. Estes, T. Gross, J.B. Hopkins, S. Kim, M. Mangel, S.D. Newsome, M.M. Pires, L. Rudolf, A.O. Shelton, and P.V. Wheatley for many helpful comments and discussions. This work was funded by an Institute of Geophysics and Planetary Physics research grant to JDY, a UC-Santa Cruz Committee-On-Research grant to PLK, a National Science Foundation Graduate Student Fellowship (NSF-GRF) to JDY, and a Fundação de Amparo à Pesquisa do Estado de São Paulo (FAPESP) grant to PRG. 


\section{$2.4 \quad$ Figures}

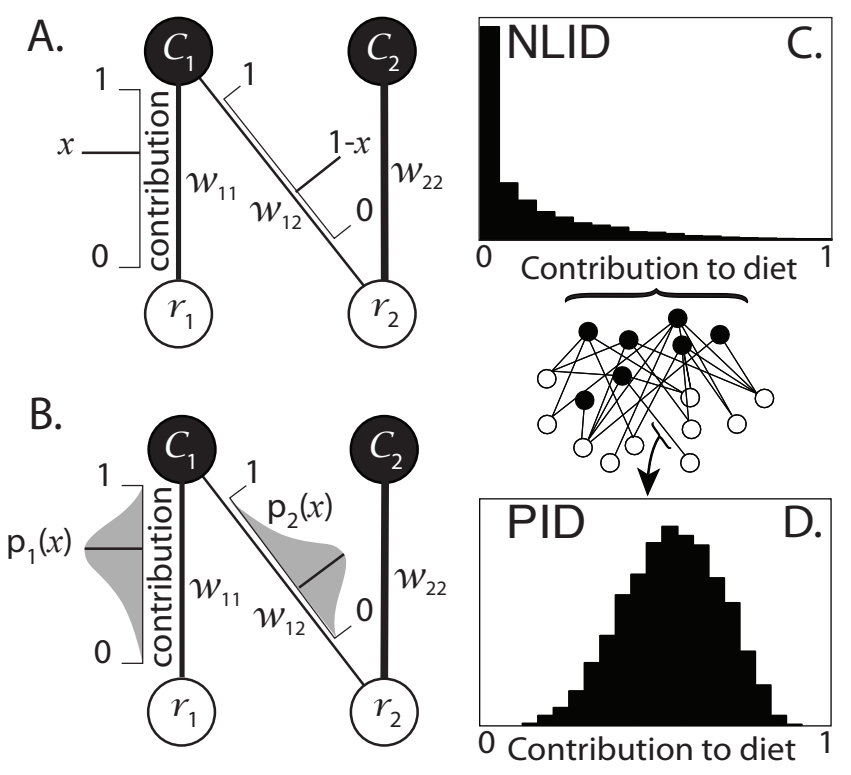

Figure 2.1: A. In this network subgraph, $\mathcal{C}_{1}$ and $\mathcal{C}_{2}$ are consumers, while $r_{1}$ and $r_{2}$ are resources. The proportional contributions of $r_{1}$ and $r_{2}$ to the diet of predator $\mathcal{C}_{1}$ are represented by the point estimates $x$ and $1-x$, for links $w_{11}$ and $w_{12}$, respectively. Because $\mathcal{C}_{2}$ is only linked to $r_{2}$ at link $w_{22}, r_{2}$ represents the entire diet of $\mathcal{C}_{2}$. B. Weights are illustrated as probability distributions $p_{1}(x)$ and $p_{2}(x)$. Individual draws from $p_{1}(x)$ and $p_{2}(x)$ must sum to unity. C. The variance among links of a given foodweb can be represented by the Network-Level Interaction Distribution (NLID), which is characteristic of the entire food-web. D. The variance within a link of a given food-web is represented by Pairwise Interaction Distributions (PIDs), which are unique to each link. 

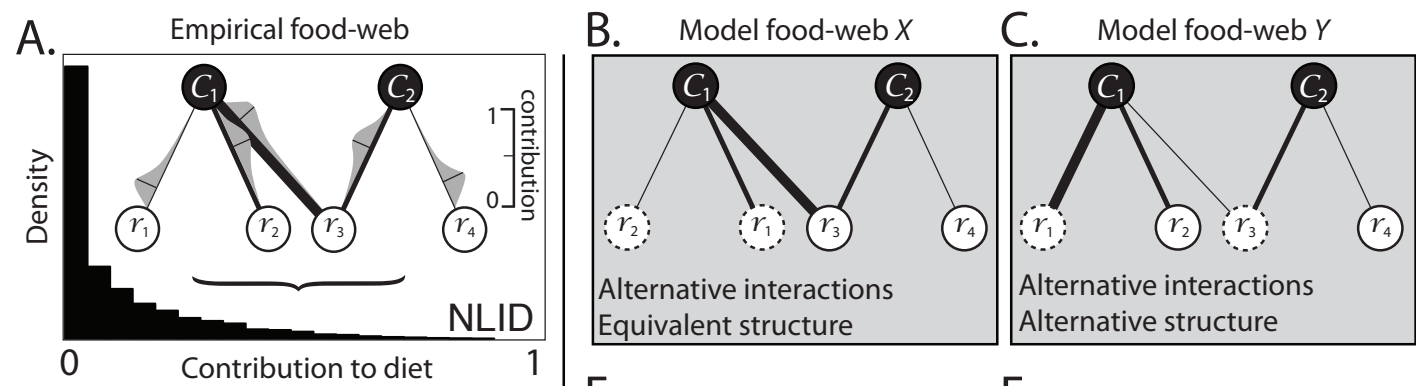

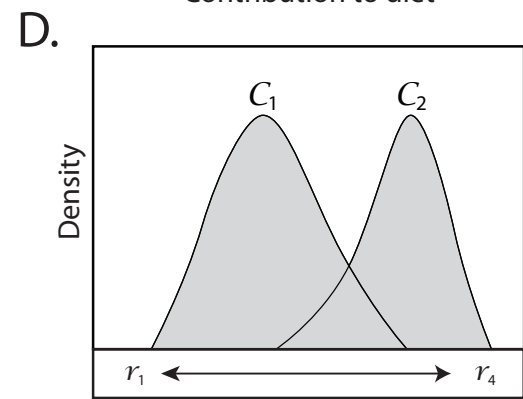

Resource Niche Axis

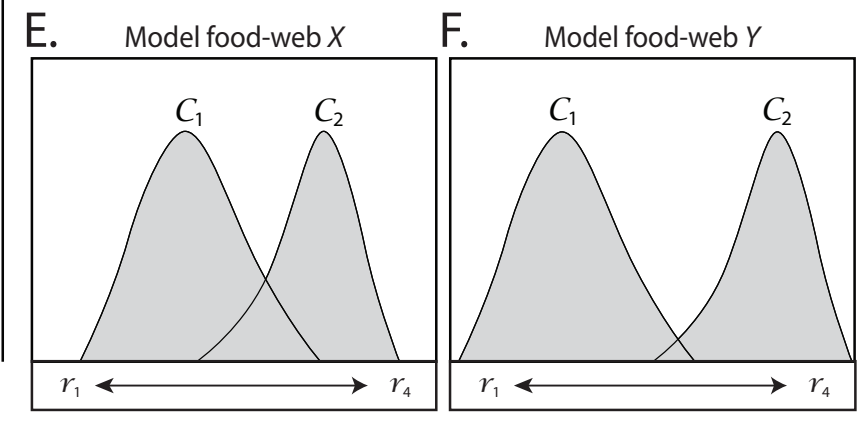

Resource Niche Axis

Figure 2.2: A. The variance among links of an empirical food-web (with 2 predators: $\mathcal{C}_{1}$ and $\mathcal{C}_{2}$, and 4 prey: $\left.r_{1}-r_{4}\right)$ is represented by both pairwise interaction distributions for each link, and a network-level interaction distribution for the system. Link thickness represents the median link-strength, shown with the vertical bar in each pairwise interaction distribution. B-C. Both model food-webs $X$ and $Y$ have link-strengths drawn from the NLID. In model food-web $X$, the weights are distributed similar to the median weights of the empirical web (i.e. equivalent structure), but they are distributed differently among nodes (i.e. alternative interactions). In model $Y$, the weight structure is different than that of the empirical web (i.e. alternative structure), and necessarily, the distribution of weights among nodes differs as well (i.e. alternative interactions). Dotted nodes indicate those with link-strengths that differ from the median link-strengths of the empirical food-web. D-F. Given a single niche axis based on a continuous resource trait (e.g. body size) where prey are ranked from $r_{1}$ to $r_{4}$, distributions of resource use are shown for the two consumers. Representations of dietary overlap illustrate higher similarity in diet between predators of the empirical system and model $X$, whereas the consumers in model $Y$ have little dietary overlap, despite sharing the same NLID. 


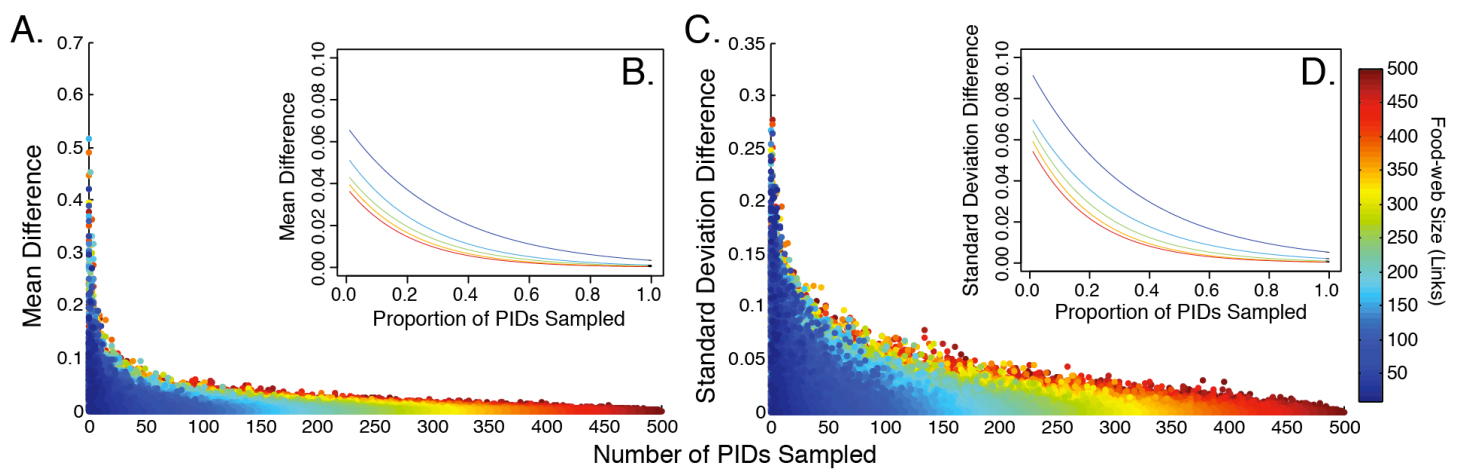

Figure 2.3: A. Numerical results of the absolute difference in means of the true and estimated NLIDs vs. the number of PIDs sampled. The size of the theoretical foodwebs are measured by the number of links, and denoted by the color gradient. B. The $\mathrm{y}$-axis is as in panel A., and the x-axis denotes the proportion of PIDs sampled across model food-webs of varying size. Exponential curves $\left(y=a \mathrm{e}^{b x}\right)$ were fitted to binned numerical results, where each bin represents an increase in ecosystem size by 100 links. C. Numerical results of the absolute difference in standard deviation of the true and estimated NLIDs vs. the number of PIDs sampled. D. The y-axis is as in C.; the x-axis and exponential curves are plotted as in B. See Table S2.2 for estimated values of $a$ and $b$ for each model fit. 
A.

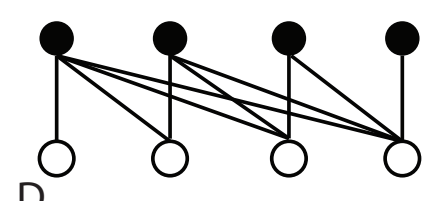

D.

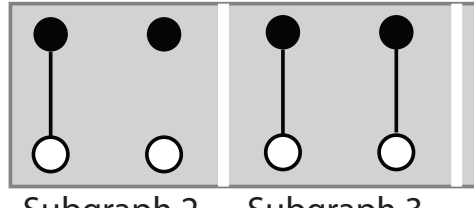

B.

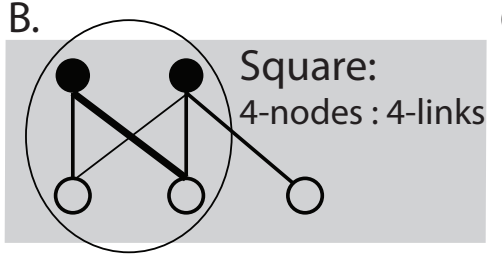

C.

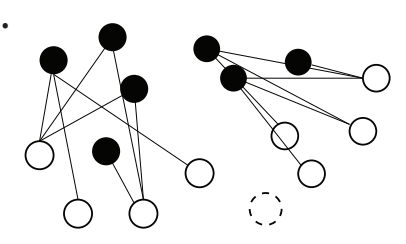

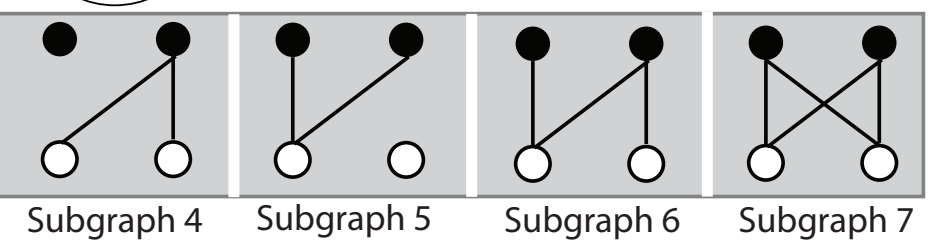

Figure 2.4: A. A nested pattern of interaction, where the consumers (black circles) towards the right consume prey (white circles) that are subsets of the consumers towards the left. B. A network square (circled), with four nodes connected to four links. Compartmentalization is both a function of the average density of squares relative to a fully connected network, and network connectance. In this example, at high cutoff values, the density of squares $\rightarrow 0$, while connectance $>0$, resulting in a modularity value of -1 (see Eq. 3.2). C. Two network components and an isolated node (stippled). D. Network Subgraphs 2 through 7. Subgraph 1 is represented by 2 predators and 2 prey not connected by interactions (not shown). 

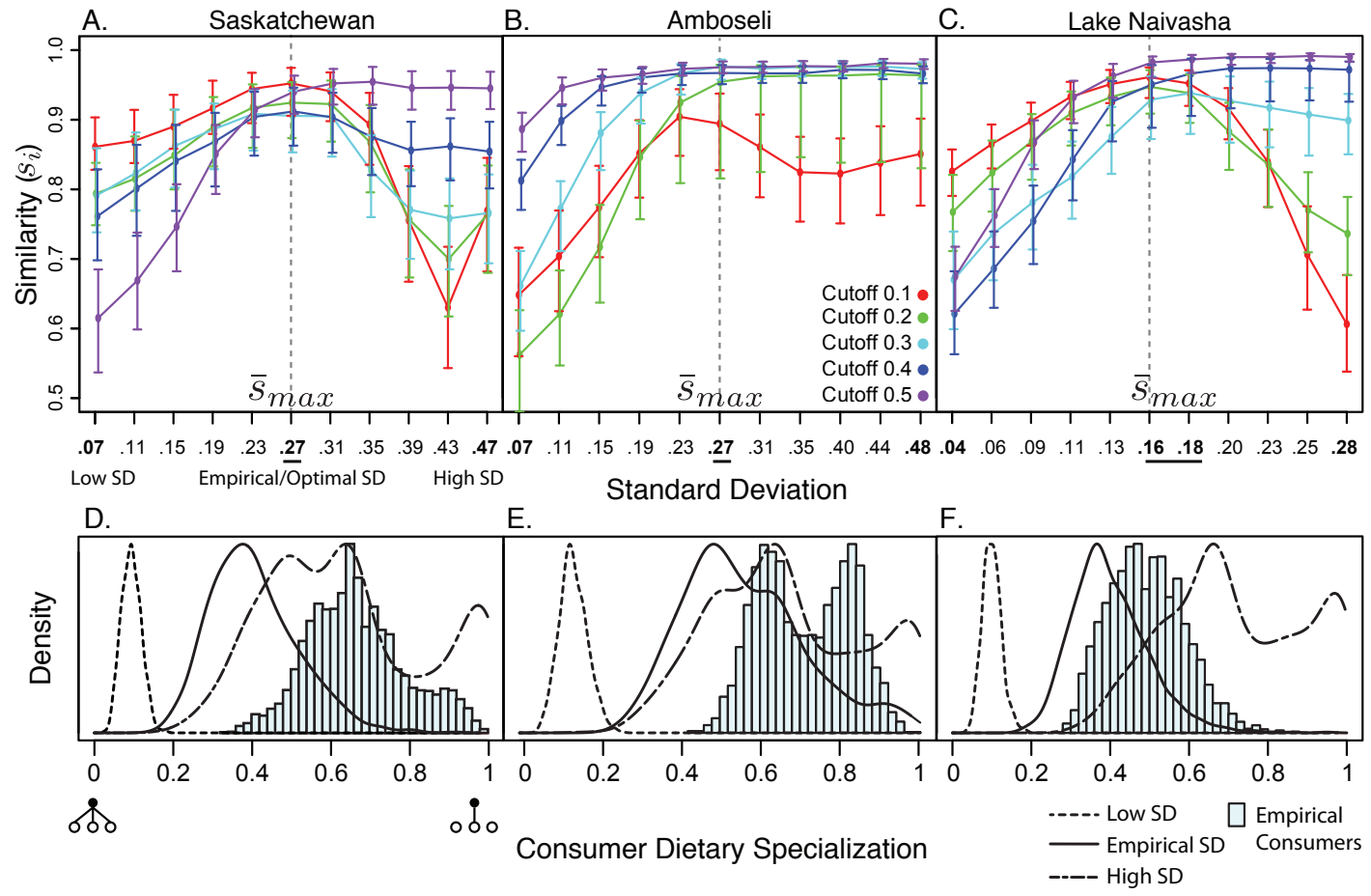

Figure 2.5: A-C. Sensitivity analyses of the Saskatchewan, Amboseli, and Lake Naivasha food-web ensembles, respectively. Similarity values for each cutoff $\left(s_{i}\right)$ for Model 2 are on the $\mathrm{y}$-axis, and the $\mathrm{x}$-axis ranges from a lower standard deviation (SD) than the empirical SD to a higher SD (such that the empirical SD is central to the range; stippled vertical line). The colored data points represent the median similarity indices for each cutoff value (legend), while the top and bottom whiskers denote the $25^{\text {th }}$ and $75^{\text {th }}$ percentiles, respectively. The underlined values mark the standard deviation corresponding to the highest average similarity across cutoff values. For all systems, the highest average similarity is close to or exactly matches the empirical standard deviation. D-F. Distributions of consumer specialization for low, optimal, and high SD (lines), and for the empirical consumers (histograms), for Saskatchewan, Amboseli, and Naivasha, respectively. When NLID SD is altered to low values, the model food-web is populated with generalist consumers. When NLID SD is altered to high values, the food-web is populated with intermediate to strong specialists. When NLID SD is held at the optimal value, consumers with intermediate values are most common. 


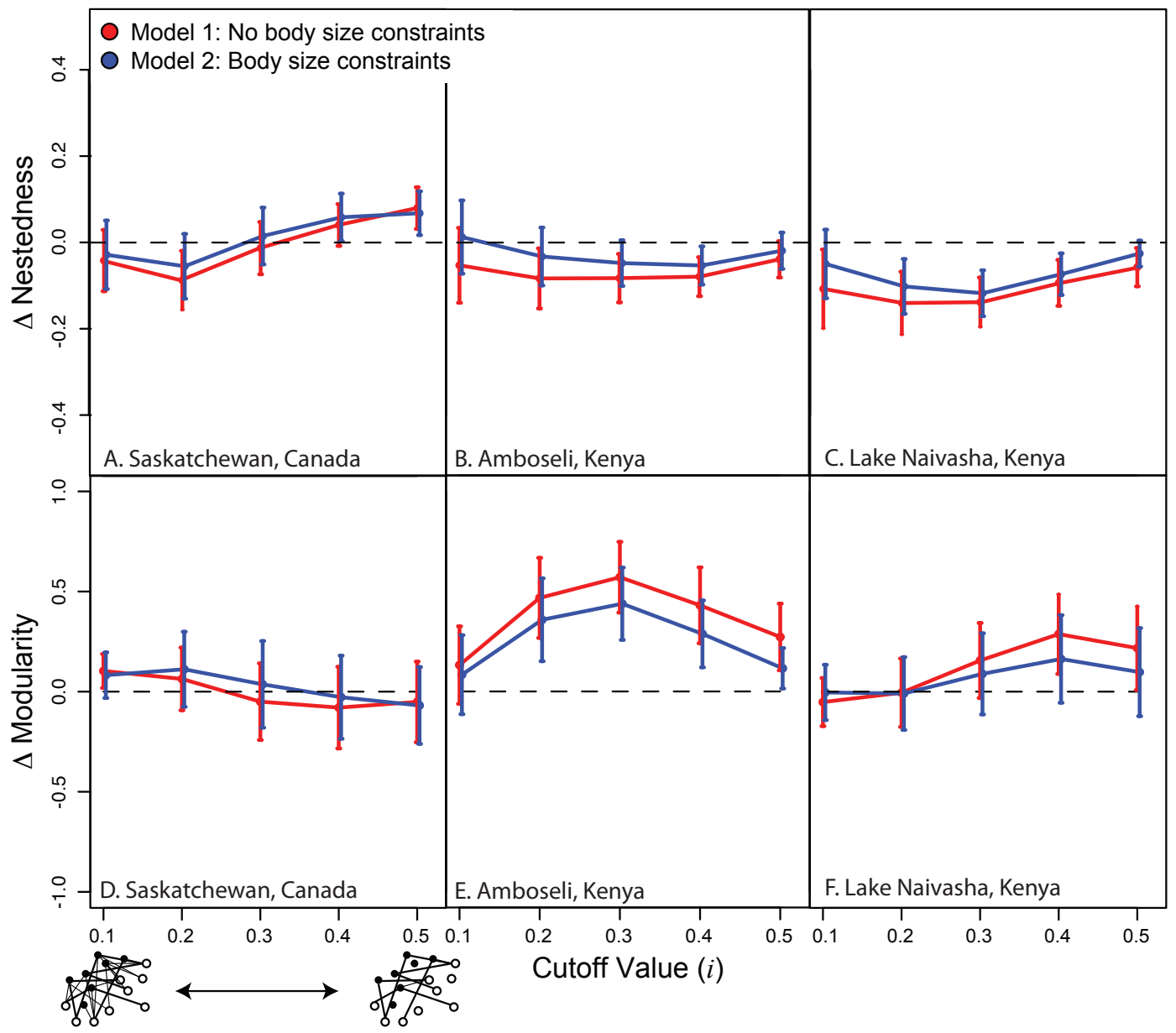

Figure 2.6: First row: the difference $(\Delta)$ in nestedness (empirical - model values) for $\mathbf{A}$. Saskatchewan, Canada, B. Amboseli, Kenya, and C. Lake Naivasha, Kenya vs. cutoff values 0.1 to 0.5 . Second row: the difference $(\Delta)$ in modularity (empirical - model values) for D. Saskatchewan, Canada, E. Amboseli, Kenya, and F. Lake Naivasha, Kenya vs. cutoff values 0.1 to 0.5 . Low cutoff values correspond to food-webs with both weak and strong interactions; high cutoff values correspond to the same food-webs with only the strong interactions intact. Difference values calculated using Model 1 are colored red; difference values calculated using Model 2 are colored blue. Whiskers denote the root mean square of the empirical and model standard deviations. The dotted line denotes the value at which there is no difference between the empirical and theoretical model nestedness or modularity values; the network illustration demonstrates the elimination of weak links as cutoff values increase. 


\subsection{Supplementary Material}

\subsubsection{Appendix 2.1. Isotopic systems and mixing model results}

We used the isotopic values of predators and prey from three systems: one from Saskatchewan, Canada (Fig. S2.2, [192]), and two from Kenya: Amboseli (Fig. S2.3, [87]), and Lake Naivasha (Fig. S2.4 [9]). To calculate distributions of the contribution of each prey to a predator's diet, we first accounted for trophic discrimination factors, such that isotopic fractionations that occur between trophic levels are eliminated. For both African systems, predator $\delta^{13} \mathrm{C}$ and $\delta^{15} \mathrm{~N}$ values were corrected by $-1 \pm 0.5 \%$ and $-3.5 \pm 0.7 \%$ respectively, thereby assuming that all carnivores had high-quality proteinrich diets $[159,157,156]$. For Saskatchewan, Canada, predator $\delta^{13} \mathrm{C}$ and $\delta^{15} \mathrm{~N}$ values were corrected by $-1.3 \pm 0.5 \%$ and $-4.5 \pm 0.7 \%$ respectively, more closely matching values calculated by [58] in the wolf-moose-beaver Isle Royale system. This differs from the discrimination factors used by [192].

We corrected for potential underestimates of isotopic variance for predators with low sample sizes by incorporating variance measurements for the same species in nearby systems. Measures of variance for hyenas and cheetahs in southern Kenya (unpublished data) were used as more conservative estimates of variance for the same species in Amboseli. Similarly, measures of variance for lions, leopards, and cheetah from central and southern Kenya (unpublished data) were used as more conservative

estimates of variance for the same species in Lake Naivasha (final values are reported in Table 2.1). The means and standard deviations presented are similar to the isotopic 
values of the same species from other African systems [43, 207]. In this manner, variance for under-sampled predators was increased, conservatively increasing the uncertainty represented in the posterior probability distributions calculated by mixSIR.

Link-strength distributions were assessed for predator:prey pairs in all systems (with the isotope mixing model MixSIR v.1.0.4 [122]; Fig. S2.2,2.3) with the exception of the Naivasha food-web where some browsers with very low $\delta^{13} \mathrm{C}$ values were too isotopically similar to resolve dietary information [9] and were binned as a functional group (Fig. S2.4). Because these browsers had isotopic values that differed significantly from all predators in the system, binning does not significantly influence the estimation of interaction distributions for their primary prey.

\subsubsection{Appendix 2.2. Calculation of similarity}

The application of the cutoff algorithm to both the empirical and model foodwebs results in a series of vectors $\mathbf{v}_{i}$ that describe the topological properties of food-webs represented in the ensemble across each of the $i$ cutoff values. The elements of both vectors are defined by values for: nestedness, modularity, the proportion of networks with positive connectance but no squares (where a square is formed by connecting four nodes with four links), the number of single nodes, the number of components, and the frequencies of each four-node motif. All elements in a vector are normalized to vary between 0 and 1 (except for modularity, which can vary between -1 and 1 ), thus limiting each element of the vector to a standardized range. We emphasize that the elements

of these vectors were chosen arbitrarily (other structural properties of networks could 
be included as well), such that the absolute similarity between two food-webs should be assessed relative to other food-webs measured with the same indices included in the similarity index. The degree to which a model food-web predicts the structural properties of an empirical food-web can be quantified with a similarity coefficient

$$
s_{i}=\frac{\mathbf{v}_{i, \text { empirical }} \cdot \mathbf{v}_{i, \text { model }}}{\left|\mathbf{v}_{i, \text { empirical }}\right|\left|\mathbf{v}_{i, \text { model }}\right|}
$$

where $\mathbf{v}_{i \text {,empirical }}$ and $\mathbf{v}_{i \text {,model }}$ are vectors of topological metric values corresponding to a cutoff value $i \in\{0.1: 0.5\}$ for the empirical and model networks, respectively, and $|\cdot|$ denotes the euclidean norm for the given vector $[88,169]$. The similarity index $s_{i}$ can vary between 0 and 1 ; a value of 0 indicates absolute dissimilarity, while a value of 1 indicates absolute similarity. We note that this similarity index is mathematically related to the cosine of the angle between two vectors. To assess the similarity of network ensembles, rather than a single network, we bootstrapped 1000 network vectors from both the empirical and model ensembles, thereby generating a distribution of similarity values that represent the topological equivalency between the empirical and model probabilistic food-webs.

\subsubsection{Appendix 2.3. Calculation of consumer dietary specialization}

The degree of consumer dietary unevenness, or specialization $(\epsilon)$ can be directly calculated from the mixing model results of a consumer's isotopic values (relative to those of its prey). For a detailed explanation of this metric, see [127]. We first define a

consumer dietary vector that has equally-weighted prey contribution-to-diet links as an 
generalist consumer end-member $(\gamma=\{1 / n, \ldots, 1 / n\}$, where $n$ is the number of potential prey, and where the sum of the vector equals unity), and a consumer with only a single link to one of its potential prey as a specialist consumer end-member $(\phi=\{1,0, \ldots, 0\})$. In $n$-dimensional diet-space, where $n$ represents the number of potential prey, we define the centroid by the generalist consumer. The Euclidean distance of a given consumer's diet to the centroid can then be measured. We divide this distance by the Euclidean distance from the centroid to the specialist consumer end-member such that consumers with different numbers of prey can be directly compared (Eq. 2.3). Thus, consumer dietary unevenness of a consumer $j$ can be quantified by

$$
\epsilon_{j}=\frac{\sqrt{\sum_{k=1}^{n}\left(w_{j k}-\gamma_{j k}\right)^{2}}}{\sqrt{\sum_{k=1}^{n}\left(\phi_{j k}-\gamma_{j k}\right)^{2}}},
$$

where the subscript $k$ refers to a prey item in the diet of the consumer $j$. Consumer dietary specialization is therefore constrained to vary between 0 and 1 , where 0 represents a maximally generalist consumer, and 1 represents a maximally specialist consumer. We note that the use of entropy-based unevenness measurements $[83,19]$ result in similar estimates. 


\subsubsection{Tables}

\begin{tabular}{lcccc} 
Species & $\delta^{13} \mathrm{C}$ & $\delta^{13} \mathrm{C} \mathrm{SD}$ & $\delta^{15} \mathrm{~N}$ & $\delta^{15} \mathrm{~N} S \mathrm{~S}$ \\
\hline Amboseli & & & & \\
Panthera leo & -9.04 & 1.67 & 11.07 & 0.45 \\
Crocuta crocuta & -9.16 & 0.89 & 12.16 & 1.09 \\
Acinonyx jubatus & -17.21 & 1.95 & 10.07 & 1.02 \\
\hline Naivasha & & & & \\
Panthera leo & -7.21 & 1.64 & 6.30 & 0.44 \\
Crocuta crocuta & -8.74 & 0.89 & 7.70 & 1.10 \\
Panthera pardus & -8.61 & 0.51 & 6.70 & 2.04 \\
Acinonyx jubatus & -14.21 & 1.95 & 6.60 & 1.02 \\
\hline
\end{tabular}

Supplementary Table 2.1: Mean and standard deviations (SDs) of isotopic values for African predators and prey that were used to assess link-strengths with the Bayesian isotope mixing model MixSIR. See Appendix 2.1 for details. 


\begin{tabular}{lcc} 
System size range (\# links) & $a$ & $b$ \\
\hline Mean Error & & \\
$1: 100$ & 0.06 & 3.02 \\
$101: 200$ & 0.05 & 3.88 \\
201:300 & 0.05 & 4.24 \\
301:400 & 0.04 & 4.74 \\
$401: 500$ & 0.04 & 5.19 \\
\hline SD Error & & \\
$1: 100$ & 0.09 & 2.86 \\
$101: 200$ & 0.08 & 3.87 \\
$201: 300$ & 0.07 & 4.30 \\
$301: 400$ & 0.06 & 4.57 \\
$401: 500$ & 0.06 & 5.06 \\
\hline
\end{tabular}

Supplementary Table 2.2: To determine the rate at which estimation of the mean and standard deviation (SD) of the NLID could be estimated as sampling effort increased, exponential curves $\left(y=a e^{b x}\right)$ were fitted to binned numerical results, where each bin represents an increase in ecosystem size by 100 links. Mean error and SD error were calculated as the difference between the true NLID mean and SD, respectively, and that estimated from subsampling PIDs. 


\begin{tabular}{|c|c|c|c|c|c|c|c|c|c|}
\hline $\begin{array}{l}\Delta \mathcal{N} \\
\text { Cutoff }\end{array}$ & $\begin{array}{c}\text { Saskatchewan } \\
\text { df }\end{array}$ & $\mathrm{t}$ & $p$ & $\begin{array}{l}\text { Amboseli } \\
\text { df }\end{array}$ & $\mathrm{t}$ & $p$ & $\begin{array}{c}\text { Naivasha } \\
\text { df }\end{array}$ & $\mathrm{t}$ & $p$ \\
\hline 0.1 & 57.7 & -0.62 & 0.54 & 57.9 & -3.87 & $<0.01^{*}$ & 57.9 & -4.08 & $<0.01^{*}$ \\
\hline 0.2 & 51.7 & -1.97 & 0.05 & 54.8 & -0.507 & 0.61 & 57.2 & -0.68 & 0.52 \\
\hline 0.3 & 57.9 & -1.31 & 0.20 & 53.9 & -2.53 & $0.01^{*}$ & 55.2 & -1.81 & 0.07 \\
\hline 0.4 & 55.9 & -2.35 & $0.02^{*}$ & 56.6 & -0.06 & 0.95 & 57.0 & -2.24 & $0.03^{*}$ \\
\hline 0.5 & 56.8 & 1.44 & 0.16 & 48.3 & -2.60 & $0.01^{*}$ & 57.4 & -1.57 & 0.12 \\
\hline $\begin{array}{l}\Delta \mathcal{M} \\
\text { Cutoff }\end{array}$ & $\begin{array}{c}\text { Saskatchewan } \\
\text { df }\end{array}$ & $\mathrm{t}$ & $p$ & $\begin{array}{l}\text { Amboseli } \\
\text { df }\end{array}$ & $\mathrm{t}$ & $p$ & $\begin{array}{c}\text { Naivasha } \\
\text { df }\end{array}$ & $\mathrm{t}$ & $p$ \\
\hline 0.1 & 53.0 & 2.25 & $0.03^{*}$ & 57.4 & 1.44 & 0.16 & 54.4 & -1.49 & 0.14 \\
\hline 0.2 & 57.9 & -0.05 & 0.96 & 48.6 & -0.61 & 0.55 & 57.0 & 1.21 & 0.23 \\
\hline 0.3 & 45.1 & -1.63 & 0.11 & 57.6 & 3.30 & $<0.01^{*}$ & 57.9 & 0.25 & 0.81 \\
\hline 0.4 & 57.2 & -0.63 & 0.53 & 56.2 & 1.55 & 0.13 & 52.7 & 2.70 & $<0.01^{*}$ \\
\hline 0.5 & 50.8 & -1.02 & 0.31 & 52.2 & 4.49 & $\ll 0.01^{*}$ & 54.3 & 3.20 & $<0.01^{*}$ \\
\hline
\end{tabular}

Supplementary Table 2.3: Welch t-test results for $\Delta \mathcal{N}$ and $\Delta \mathcal{M}$ between Models 1 and 2. Model 1 does not include body size constraints, while Model 2 does include body size constraints (refer to Fig. 6, main text). For the most part, the mean values of Model 1 and 2 are not significantly different, with some exceptions indicated by the asterisk $\left(^{*}\right)$. 


\subsubsection{Figures}

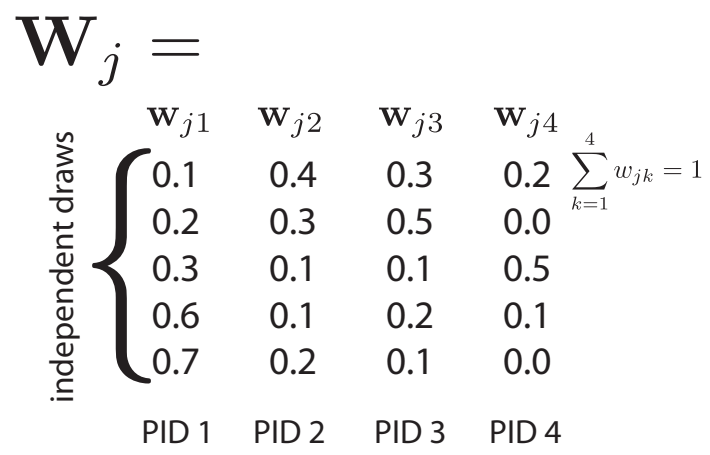

Supplementary Figure 2.1: An illustration of the proportional contribution to diet matrix $\mathbf{W}_{j}$ for predator $j$. Each column $\mathbf{w}_{j k}$ is a vector that describes numerically a single PID in the probabilistic network. Equivalently, columns represent the probabilistic linkstrength distribution connecting the predator $j$ to prey $k$, and account for variability within a single link. By contrast, each row is an independent draw from a Bayesian isotope mixing model, representing a single set of link-strengths for a potential quantitative food-web in the probabilistic ensemble. Because the rows describe a potential set of values for the proportional contribution of prey $k=1$ to 4 , they are constrained to sum to one. As a whole, the matrix $\mathbf{W}_{j}$ is a numeric description of a multivariate posterior probability distribution, the columns of which represent marginal distributions. 
Saskatchewan Predator Diets
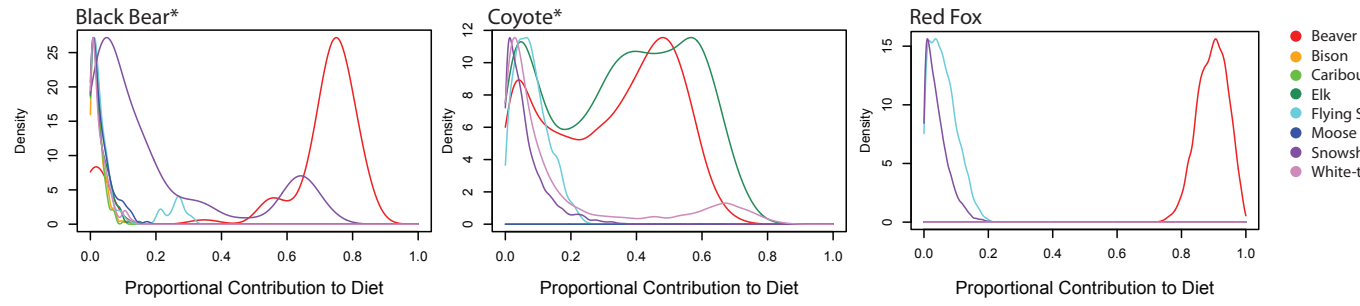

- Elk

- Flying Squirrel

- Snowshoe Hare

White-tailed Deer
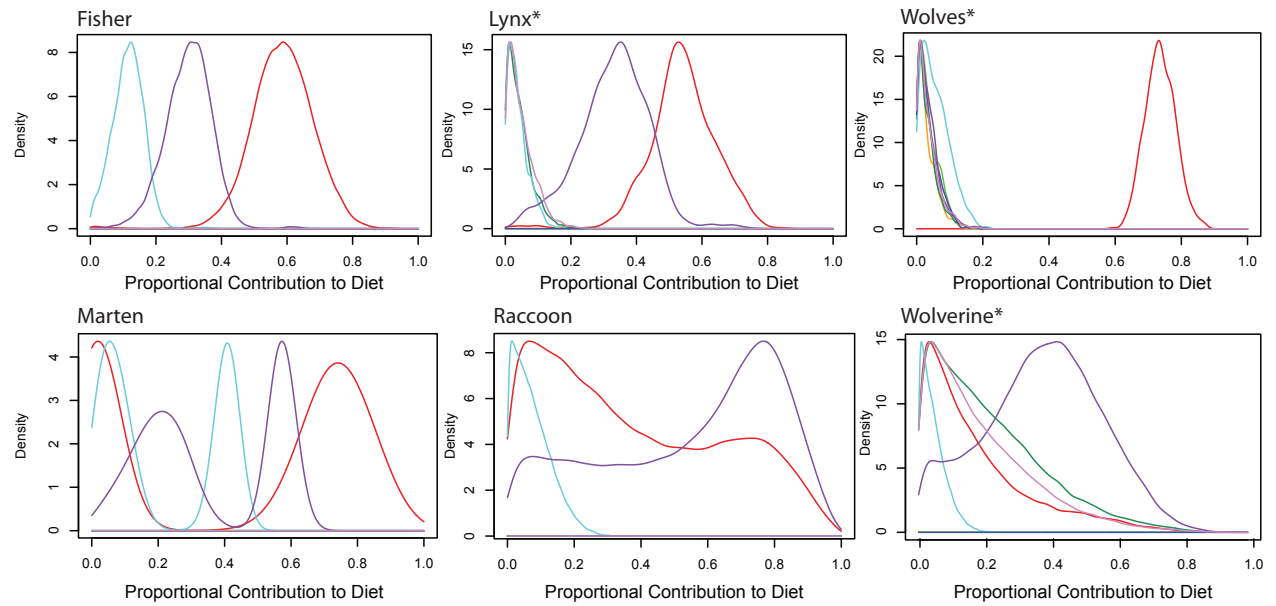

Supplementary Figure 2.2: Proportional contribution of prey to the diet of predators (PIDs) in the Saskatchewan system. PID estimates are from the Bayesian isotope mixing model MixSIR. Predator:prey PIDs that are not reported indicate the presence of a forbidden interaction. Species marked with stars represent the larger predators in the system; topological properties were assessed with just these predators included, and are reported in Fig. S2.6. Forbidden Links: Fox, fisher, marten, and raccoon to bison, caribou, elk, moose, white-tailed deer; coyote, lynx, and wolverine to bison, caribou, moose. 

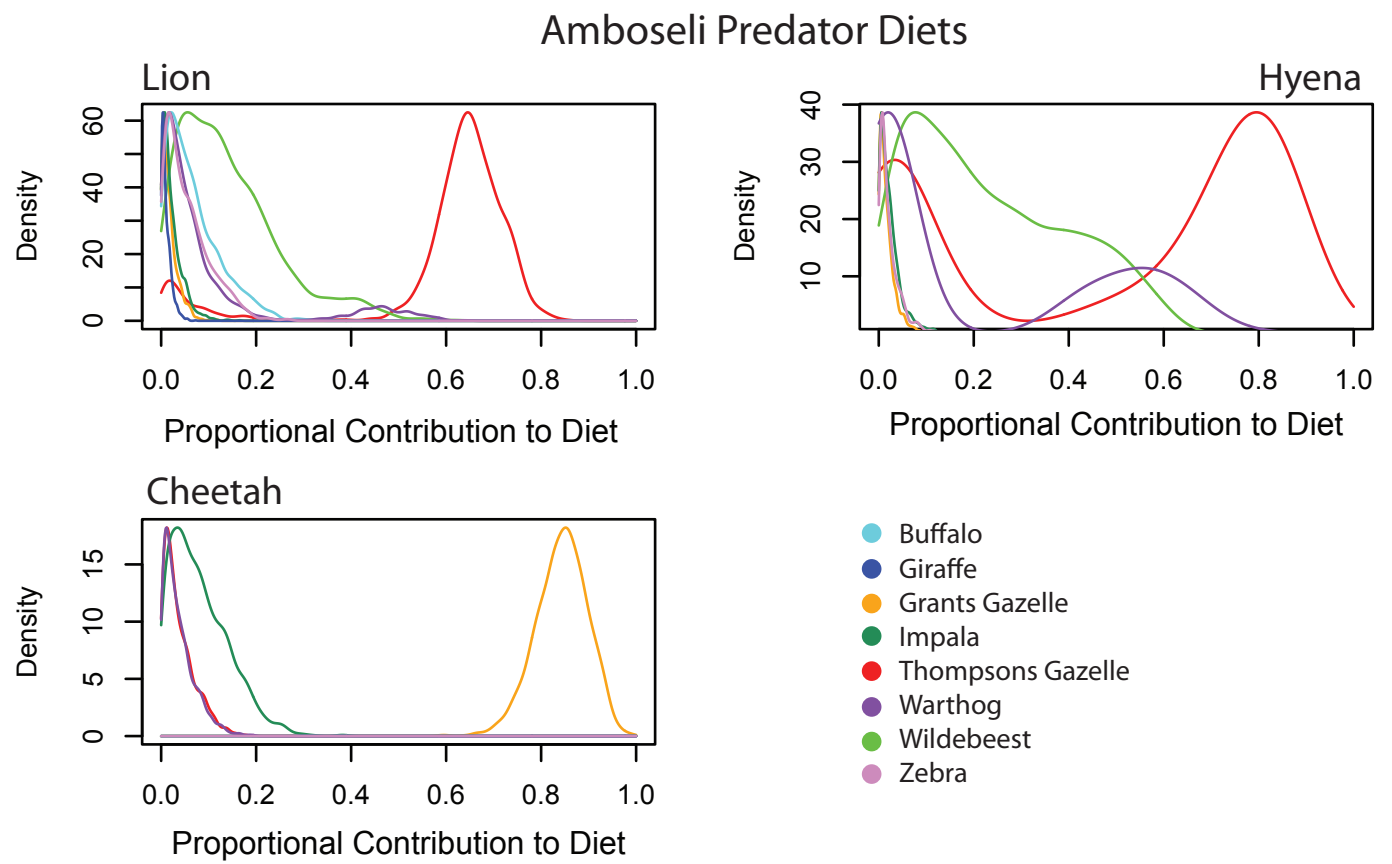

Supplementary Figure 2.3: Proportional contribution of prey to the diet of predators (PIDs) in the Amboseli system. PID estimates are from the Bayesian isotope mixing model MixSIR. Predator:prey PIDs that are not reported indicate the presence of a forbidden link. Forbidden Links: cheetah to buffalo, giraffe, wildebeest, zebra. 

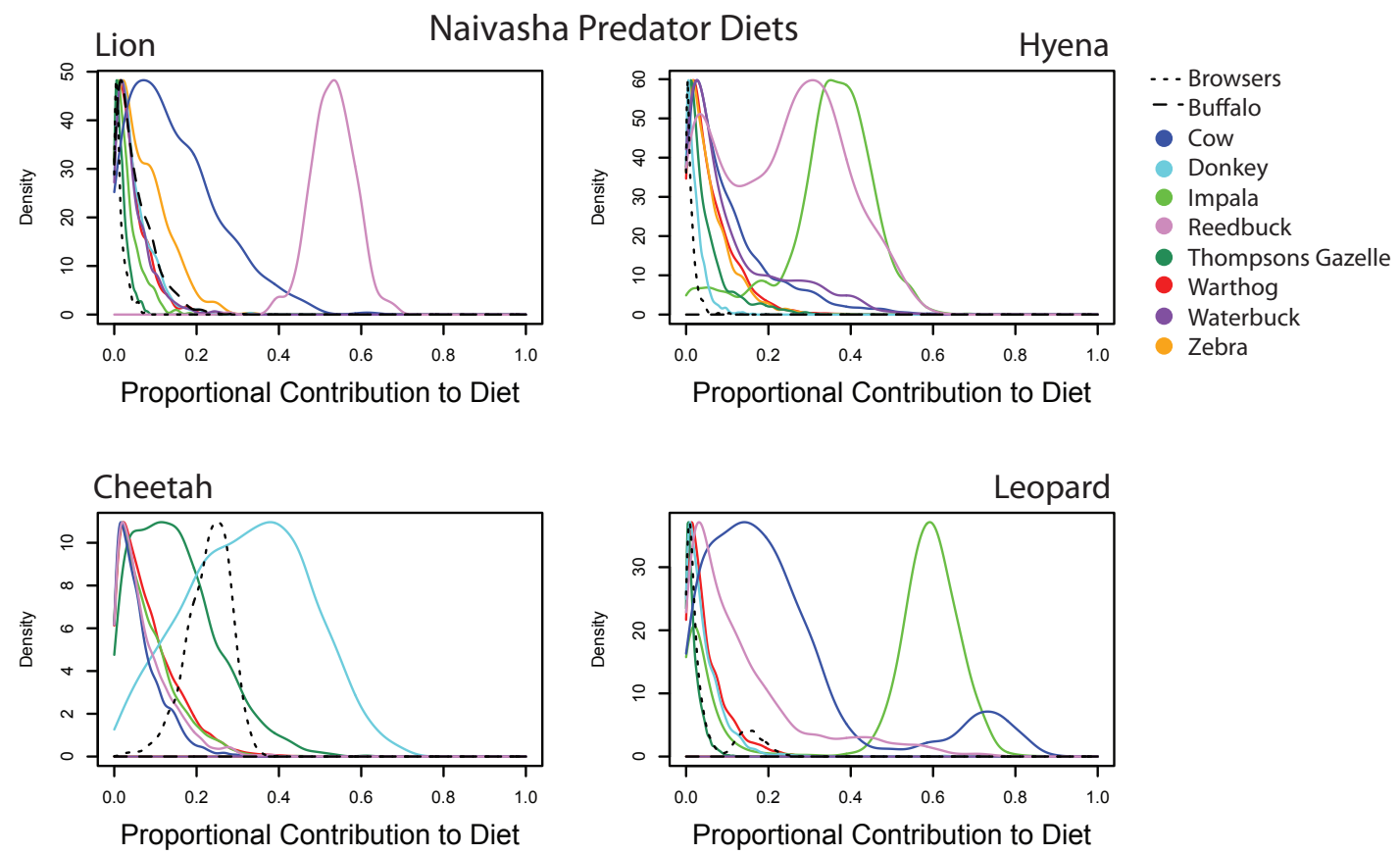

Supplementary Figure 2.4: Proportional contribution of prey to the diet of predators (PIDs) in the Naivasha system. PID estimates are from the Bayesian isotope mixing model MixSIR. Predator:prey PIDs that are not reported indicate the presence of a forbidden link. A subset of browsers were too isotopically similar to resolve dietary information, though they had values set apart from all potential predators, suggesting minimal dietary inclusion. As such, they were binned together as a 'browser' functional group and include: Steenbok, Dik Dik, Baboon, Grey Duiker, Klipspringer, Bushbuck, and Eland. Forbidden Links: cheetah and leopard to buffalo, waterbuck, and zebra. 

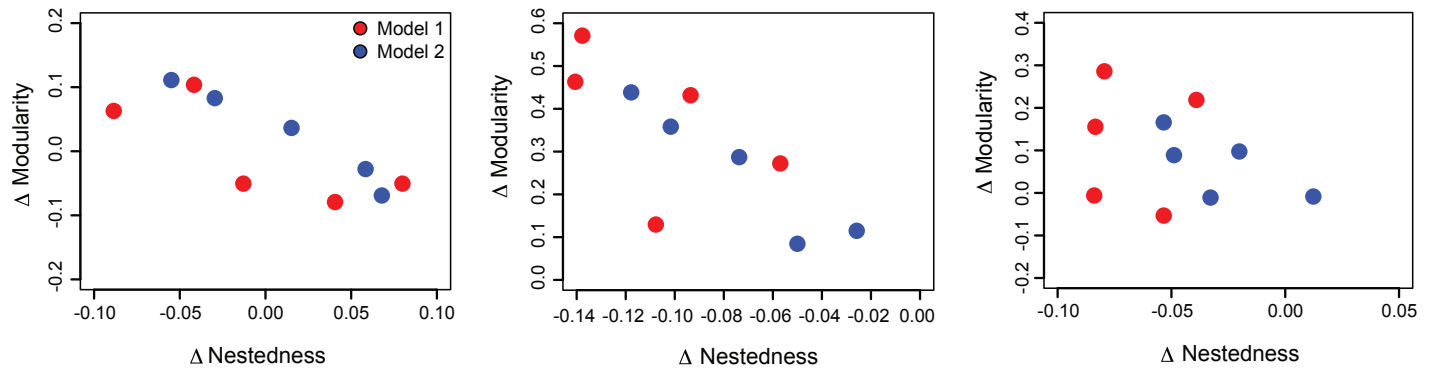

Supplementary Figure 2.5: The difference $(\Delta)$ in modularity (empirical - model values) vs. the difference in nestedness for the three isotopic food-webs. Model 1 does not incorporate body size constraints, while Model 2 does incorporate body size constraints. In each system, there is a strong negative relationship between modularity and nestedness for Model 2 (though this relationship is not statistically significant for the Naivasha system). Saskatchewan Model 1: $\mathrm{R}^{2}=0.47, \mathrm{p}>0.05$, Model 2: $\mathrm{R}^{2}=0.96, \mathrm{p} \ll 0.05$. Amboseli Model 1: $\mathrm{R}^{2}=0.09 ; \mathrm{p}>0.05$, Model 2: $\mathrm{R}^{2}=0.86 ; \mathrm{p}<0.05$. Naivasha Model 1: $\mathrm{R}^{2}=-0.33 ; \mathrm{p}>0.05$, Model 2: $\mathrm{R}^{2}=0.25 ; \mathrm{p}>0.05$. 


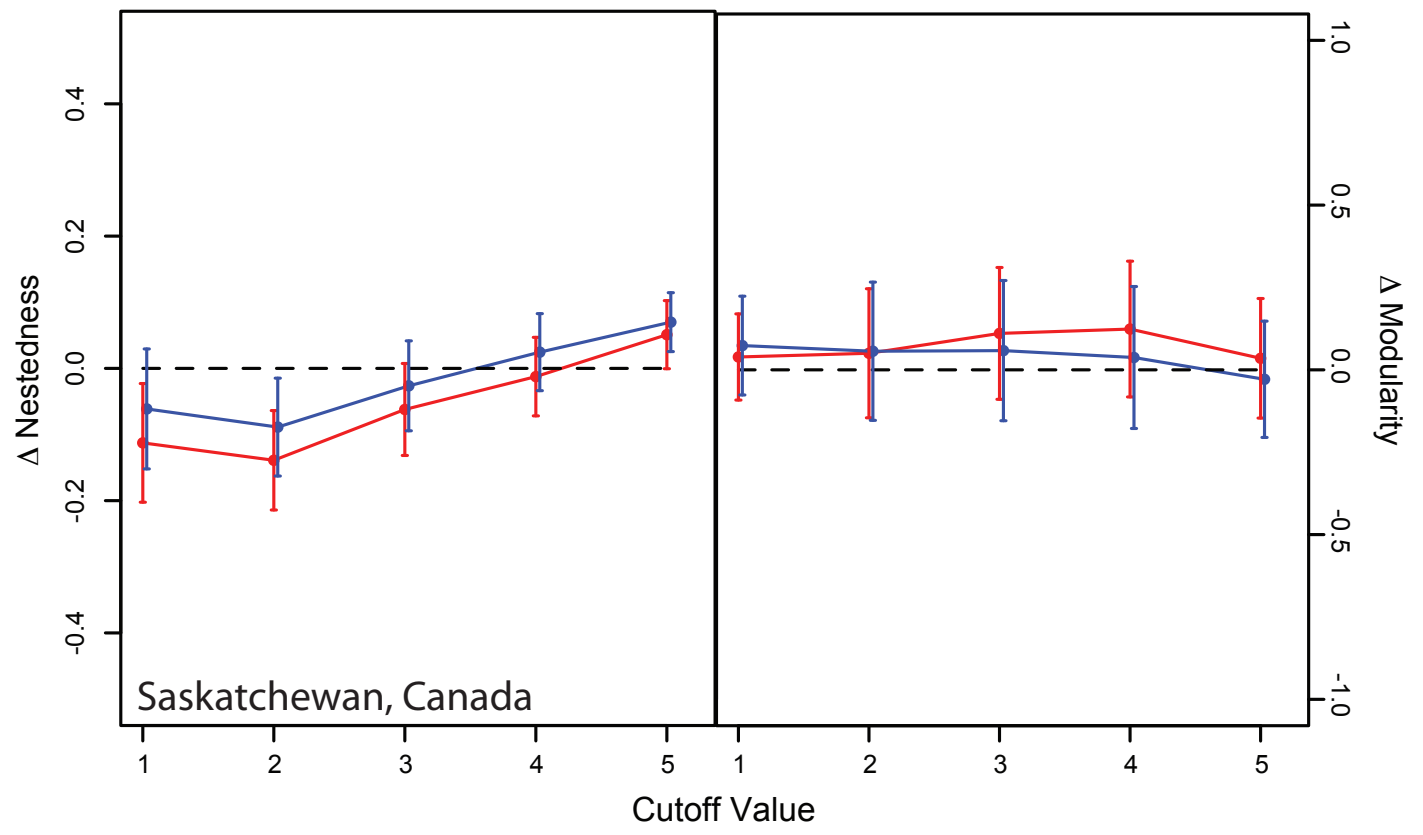

Supplementary Figure 2.6: The difference $(\Delta)$ in nestedness (empirical - model values) for Saskatchewan, Canada, with only the large predators (and predators that primarily consume larger prey) included in the food-web ensemble. Predators include: Black bear, Coyote, Lynx, Wolves, and Wolverine. These results are qualitatively similar to those obtained for the full food-web, suggesting that the structure of interactions is fairly robust to modification. 


\section{Chapter 3}

\section{The impact of climate change on the}

\section{structure of Pleistocene mammoth}

steppe food-webs

J D Yeakel, P R Guimarães Jr., H Bocherens, P L Koch 


\section{Abstract}

Community dynamics are impacted by the patterns of species interactions that shape food-web networks. However, an understanding of how these patterns change over long periods of time is typically limited to extant communities, and is particularly problematic for long-lived species. Here we use ratios of stable isotopes to reconstruct patterns of trophic interactions for six independent mammalian communities that inhabited the mammoth steppe environment, a unique Pleistocene ecotope that spanned western Europe to eastern Alaska. We use a Bayesian mixing model to quantify the proportional contribution of prey to the diets of local predators, and assess how the structure of trophic interactions change from both Beringia to Europe, and across the Last Glacial Maximum (LGM), a global climatic event that severely impacted mammoth steppe mammalian communities. We find that large felids had diets that were more constrained than other co-occurring predators, and largely influenced by an increase in Rangifer abundance after the LGM. Moreover, the structural organization of Beringian and European communities strongly differed. While European communities did not have modular structures (where species interactions are compartmentalized), the Beringian food-web was highly modular, particularly before the LGM. This modularity was lost during the LGM, and partially recovered after the glacial retreat. We suggest that the changing structure of Beringian food-webs across the LGM may be explained by Beringia's insularity with respect to surrounding communities and external invaders. 


\subsection{Introduction}

The structural patterns of species interactions affect ecosystem dynamics [5], and are sensitive to external perturbations $[173,188]$. Impacts of perturbations on food-web structure may be immediate or lagged [186]; they can affect communities by reorganizing interactions [137], changing the magnitudes of interactions [89, 14], or eliminating species $[186,172]$. However, observations of community organization across a perturbation event are typically confined to short timescales and to communities with fast turn-over rates. To assess the long-term effects of external perturbations empirically, it is necessary to use paleontological or historical information [50]. The last globally-significant climatic perturbation occurred in the late Pleistocene and culminated with the Last Glacial Maximum (LGM, 26.5 to 19 kyr BP) [42], strongly impacting a mammalian community extending across the Eurasian mammoth steppe [208, 59], an environment with no modern analogue [68]. An examination of species interactions across this climatic event is well-suited to assess the effects of global perturbations on animal communities.

Although evidence of many paleontological species interactions is irrecoverable, interactions that involve a flow of biomass are recorded in animal tissue, and can be reconstructed using stable isotope ratios [85, 128, 127, 205], and can be used to compare species' roles across the mammoth steppe environment. Moreover, a determination of larger-scale food-web structure in space across both the mammoth steppe and in time across the Last Glacial Maximum permit an examination of whether specific struc- 
tural properties characterized these ecosystems, and how they were impacted by climate change. Mammoth steppe communities were taxonomically similar across Eurasia [21], although the inherent plasticity of species' roles from Beringia to Europe is not known. Nor is it known whether generalized features of trophic systems, such as the degree of dietary specialization among consumers, varied across this expansive ecosystem. Global ice sheets attained their maximum volume during the LGM [42], separating warmer, mesic periods before and after. This change in global climate had tremendous impact on the mammoth steppe ecosystem, eliminating temperate species (particularly in Europe), and initiating a shift from tree-covered habitats to xeric grassland-dominated habitats across Eurasia [2]. Although the mammoth steppe experienced dramatic climatic shifts during the late Pleistocene, whether such changes impacted trophic interactions or by extension community-scale structure, has not been determined.

We employ a three-pronged approach to address these issues. First, we use a Bayesian isotope mixing model to quantify the structure, magnitude, and uncertainty of trophic interactions from stable isotope ratios of mammals in six independent Eurasian predator-prey networks spanning the LGM. Second, we compare species' roles across the mammoth-steppe environment, determine whether these roles changed in response to the arrival or extinction of co-occurring species, and assess the degree of specialization within and among predator guilds. Third, we determine how community-level patterns of interaction change from Beringia to Europe, and quantify differences in network structure as a function of trophic interaction magnitude across the LGM. In tandem, these combined approaches reveal the variability of mammoth steppe predator-prey 
network structure, the degree that species' roles varied over space and time, and how these changing patterns of interaction influenced the structural properties of food-webs over long timescales.

\subsection{Materials and Methods}

\subsubsection{Study sites}

During the late Pleistocene, the mammoth steppe extended from western Europe to eastern Alaska [21]. The steppic mammalian community is noted for its species richness $[72,68]$, despite the assumed low productivity of local vegetation. This 'productivity paradox' [72] suggests that mammoth steppe vegetation differed from modern tundra-dominated flora $[68,208]$. Indeed, palynological evidence indicates that tundra and boreal vegetation retreated to isolated refugia during the height of the LGM (21-17 kyr BP) [68, 208, 35]. It is now generally accepted that before and after the LGM (the pre-Glacial and post-Glacial, respectively), mammoth steppe vegetation consisted of relatively mesic coniferous woodland mosaics in Beringia and Europe [35, 36, 124]. Evidence of forests during the LGM is restricted to southern, central, and western Europe [200]. By contrast, LGM Beringia was a nearly treeless, hyper-xeric, and highly productive steppe dominated by low-sward herbaceous vegetation [208, 210].

Mammalian communities were taxonomically similar across Eurasia [21]. From Beringia to Europe, large felids (the saber-toothed cat, Homotherium serum, in Beringia and the cave lion, Panthera spelaea, in Europe and Beringia after the pre-glacial) [11], 
brown bears (Ursus arctos), and wolves (Canis) were the dominant predators, while short-faced bears (Arctodus) were exclusive to Beringia and North America, and cave hyenas (Crocuta crocuta) were exclusive to western Eurasia and Africa. Mammoth steppe herbivores had similarly large ranges, and included wooly mammoths (Mammuthus primigenius), caribou (Rangifer tarandus), yak (Bos mutus), bison (Bison spp.), horses (Equus ferus), caprine bovids (Ovibos moschatus in Beringia, and Rupicapra rupicapra in Europe) and the wooly rhinocerous (Coelodonta antiquitatis). In contrast to Beringia, Europe hosted a diverse cervid community, including red deer (Cervus elaphus), roe deer (Capreolus capreolus), and the Irish elk (Megaloceros giganteus). Although the Beringian mammoth steppe ecosystem was not significantly influenced by humans or human ancestors before ca. $13.5 \mathrm{kyr} \mathrm{BP}[86]$, Homo neanderthalensis is known to have occupied European systems (sometimes sporadically) from ca. 300 kyr BP to 30 kyr BP $[119,48]$. Neanderthal diets in continental regions were dependent on terrestrial animals $[24,27,153]$, though their role as predators relative to co-occurring carnivores is not well understood.

\subsubsection{Estimating diet from stable isotope data}

Ratios of stable isotopes can be used to infer trophic interactions between predators and prey. Because prey isotope ratios are recorded in consumer tissues, and are robust to diagenetic alteration over long periods of time, they can be used to reconstruct historic or paleontological patterns of resource use [84, 85, 128]. If ratios of stable

carbon and nitrogen isotopes are known for both predators and potential prey, mixing 
models can be used to quantify the proportional contribution of prey to a predator's diet [122], thereby establishing a per-capita measure of mass flow between interacting species in a food-web. Values of carbon and nitrogen isotope ratios are expressed as $\delta^{13} \mathrm{C}$ and $\delta^{15} \mathrm{~N}$ respectively, where $\delta=1000\left(\left(R_{\text {sample }} / R_{\text {standard }}\right)-1\right)$ and $R={ }^{13} \mathrm{C} /{ }^{12} \mathrm{C}$ or ${ }^{15} \mathrm{~N} /{ }^{14} \mathrm{~N}$, with units of per-mil (\%o); reference standards are Vienna PeeDee belemnite for carbon, and atmospheric $\mathrm{N}_{2}$ for nitrogen.

We used previously published stable isotope datasets to reconstruct trophic interactions for six independent predator-prey networks from eastern Beringia to western Europe, before, during, and after the LGM (Fig. 3.1). The three European predatorprey networks include the Ardennes (ca. 44.7 to 28.7 kyr BP) and Swabian Jura (ca. 44.7 to 28.7 kyr BP) during the pre-Glacial, and Jura during the post-Glacial (ca. 16.9 to 14 kyr BP) $[23,24,28]$. The three Beringian predator-prey networks occurred near Fairbanks, Alaska, and date to the pre-Glacial (ca. 50 - 27.6 kyr BP), full-Glacial (ca. 27.6 to $21.4 \mathrm{ky} \mathrm{BP}$ ), and post-Glacial (ca. 21.4 to $11.5 \mathrm{kyr} \mathrm{BP}$ ) [59]. To assess the role of H. neanderthalensis in pre-Glacial European networks, we used published isotope data for western French and Belgian neanderthal specimens dated to ca. 48 to $34 \mathrm{kyr}$ BP $[27,22,153]$. Because Equus and Mammuthus isotope values are similar in the above neanderthal sites compared to those in the Ardennes and Swabian Jura [23, 24, 26], we consider an assessment of neanderthal diet from these combined assemblages to be meaningful. Accordingly, we include neanderthals as potential predators in both preGlacial European networks.

We used estimates of trophic interactions quantified from stable isotope ra- 
tios to reconstruct paleontological food-webs. Food-web networks typically consist of species (nodes) connected by trophic interactions (links). In quantitative food-webs, link-strengths quantify the relative importance of individual links in a system. To calculate link-strength distributions for each trophic interaction in a food-web, we used MixSIR (v. 1.0.4), a Bayesian isotope mixing model [122]. In this context, link-strengths represent the proportional flow of biomass from prey to predators, such that the links connecting all prey to a given predator are constrained to sum to one. Moreover, link-strength distributions account for potential individuality among predator diets (see Appendix 3.1 for details). Because Bayesian mixing models account for link-strength variance, proportional prey contributions are quantified as posterior probability distributions, thereby accounting for ecological variability, variation in trophic discrimination factors, non-unique solutions, and measurement uncertainty [122]. Accordingly, each link is described by a unique probability distribution, such that link-strengths have associated probabilities for all interactions in a food-web.

To estimate trophic interactions from stable isotope data accurately, we corrected for metabolic fractionations between consumers and prey by applying Trophic Discrimination Factors (TDFs) to each prey species (reported as the difference, $\Delta$, between predator and prey $\delta$ values). TDFs have been measured for mammalian bone collagen in a North American wolf-cervid community [58] $\left(\Delta^{13} \mathrm{C}=1.3 \%\right.$ and $\Delta^{15} \mathrm{~N}=$ $4.6 \%$ ), however $\Delta^{15} \mathrm{~N}$ values may vary between 3 to $5 \%$ [25]. To account for this potential variation, we used $\Delta^{13} \mathrm{C}=1.3 \pm 0.7 \%$, and two alternative $\Delta^{15} \mathrm{~N}$ ranges for the Beringian systems: $3.5 \pm 0.6 \%$ and $4.6 \pm 0.6 \%$, thereby accounting for the full range 
of $\Delta^{15} \mathrm{~N}$ variation observed in mammalian trophic systems. Final posterior probability distributions were then derived by sampling from the alternative posterior probability distributions for each TDF scenario. In some cases, this procedure resulted in bimodal prey-contribution distributions for a given predator (as expected); we interpret these results as alternative hypotheses of predator diet with probabilities equal to the final posterior distribution. We consider the integration of alternative $\Delta^{15} \mathrm{~N}$ values as a conservative estimate of predator diet, accurately reflecting the uncertainty of mixing model approaches.

By contrast, values of $\Delta^{15} \mathrm{~N}$ larger than $3.5 \pm 0.6 \%$ could not be used for the European systems, as they unrealistically removed hyper-carnivorous predator taxa, such as Crocuta, from the mixing space. Thus, we assume this TDF range is the highest that can be applied to these ecosystems. Although $\Delta^{15} \mathrm{~N}$ variance is not well understood, the application of this lower value relative to those used for Beringian systems relies on the assumption that the most abundant large-bodied prey are included in the analysis [28]. We acknowledge that smaller prey, included rodents and birds, are not well represented in this analysis (though Spermophilis and Lepus are included in the Jura post-Glacial site). Including prey that account for occasional, but not substantial, trophic interactions (e.g. interactions between large-bodied consumers and small-bodied prey) decreases the accuracy by which other interactions are measured.

Dietary protein quality is known to influence $\Delta^{15} \mathrm{~N}$; low protein quality diets (where the amino acid profiles of the foods are not complimentary to those of the consumer) tend to result in higher values of $\Delta^{15} \mathrm{~N}[157,156]$. The degree that neanderthals 
relied on meat is unknown, though most workers agree that meat was an important dietary component. Accordingly, we used two alternative values of $\Delta^{15} \mathrm{~N}$ to account for potential differences in dietary protein quality. If neanderthals had high protein quality diets, $\delta^{15} \mathrm{~N}$ TDF $\approx 3.5 \%$ [ $[69,156]$, whereas lower protein quality diets (e.g. supplemented by fruits or grains) produce $\delta^{15} \mathrm{~N} \mathrm{TDF} \approx 4.6 \%$ [157, 156]. Results for these alternative TDFs for neanderthal diets are reported separately (Fig S3).

Herbivores from each paleontological assemblage are assumed to be potential prey for all co-occurring predators. Although adults of large-bodied taxa such as Mammuthus and Coelodonta would escape strong predation from most consumers, they may represent important scavenged resources, and are included as potential prey for smaller species. In contrast, cave bears (Ursus spelaeus) are only included as potential prey for Panthera, Crocuta, and H. neanderthalensis in European systems. This distinction is supported by evidence for strong herbivory among cave bears [26], and for predation on cave bears by large-bodied carnivores [196, 37].

\subsubsection{Statistical comparisons among sites}

Although there were strong taxonomic similarities from Beringia to Europe, the plasticity of trophic interactions across the mammoth steppe is unknown. To compare trophic interactions in pre- and post-Glacial Beringia and Europe (the full-Glacial is only represented in Beringia), we compared link-strength posterior probability distributions for predator-prey taxonomic groups present in both locations. If there were strong similarities between predator diets across Eurasia, we expected a given preda- 
tor's link-strength distributions to be similar across locations. Predator groups present in Beringia and Europe include felids (Homotherium and Panthera spelaea in pre-Glacial Beringia and Europe, and Panthera spelaea in post-Glacial Beringia and Europe), Canis, and Ursus. Prey groups include Equus, Bison, Mammuthus, caprine bovids (Ovibos in Beringia, and Rupicapra in Europe during the pre-Glacial), and Rangifer.

To assess whether the link-strength distributions describing species interactions changed from Europe to Beringia, we plotted the numerical estimates of preycontribution-to-diet values for each predator-prey interaction during the pre-Glacial as well as the post-Glacial time periods. We then used a bivariate kernel-density algorithm [56] to determine whether the majority of dietary estimates were similar over space. If the dietary composition of a given predator taxonomic group was similar in Europe and Beringia, the highest densities of dietary estimates would fall on the 1:1 line (Fig. 3.2). In contrast, if diets were strongly divergent among regions, peak densities would fall closer to the $\mathrm{x}$ - or $\mathrm{y}$-axis. This approach not only allows for an assessment of dietary similarity between predators between Europe and Beringia, but is also well-suited for determining whether such relationships changed over time.

\subsubsection{Predator specialization and prey selectivity}

To evaluate predator dietary strategies, we quantified consumer dietary unevenness with respect to the proportional contribution of prey to predator diets (Fig. 3.3). Consumer dietary unevenness measures predator dietary specialization irrespective

of herbivore abundance. Accordingly, specialization values $(\epsilon)$ are comparable between 
predators in an ecosystem, but should be used with caution when comparing between ecosystems, where prey abundance distributions may differ. We use a scaled Shannon index to measure specialization for each predator species (cf. $[83,19])$, such that

$$
\epsilon=\frac{H-H_{\text {even }}}{H_{\text {uneven }}-H_{\text {even }}}, \text { and } H=-\sum_{j=1}^{n} p_{j} \log p_{j},
$$

where $n$ is the number of prey for a given predator, $p_{j}$ is the proportional contribution of prey $j$, and $H_{\text {even }}$ and $H_{\text {uneven }}$ correspond to the theoretical minimum and maximum of $H$ for a given predator, respectively; this measure approximates other dietary specialization metrics $[127,205]$. Accordingly, if a predator consumes a single prey to the exclusion of others, $\epsilon \approx 1$; if a predator consumes similar proportions of all available prey, $\epsilon \approx 0$.

Because the three Beringian sites are from the same locality, they are wellsuited to assess both the relative importance of prey species, as well as changes in dietary specialization of the predator guild, across the LGM. To determine whether the relative importance of different prey species changed across the LGM, we quantified the relative contribution of prey to the predator guild by sampling link-strength distributions connecting prey across each predator species. These across-predator distributions represent the contribution of each prey to the predator guild (Fig. 3.4A). Because prey abundance is expected to play an important role in predator diet, changes in these measures across the Beringian temporal sequence reflect either changes in relative prey abundance, predator foraging strategies, or a combination of the two factors. Moreover, measures of across-predator prey contribution permit an assessment of dietary special- 
ization of the predator guild over time. We calculated dietary specialization for the predator guild as a whole $\left(\epsilon_{g}\right.$; using the methods described above, applied to the acrosspredator prey-contribution distributions) to determine whether reliance on available prey changed across the LGM (Fig. 3.4B; results for European systems are presented in Fig. S3.4).

\subsubsection{Community-level network analysis}

We used a Bayesian isotope mixing model to quantify link-strength posterior probability distributions. These distributions quantify the probability that a link has a given link-strength, such that the food-web is inherently probabilistic. To account for link-strength probabilities in our analysis of food-web structure, we treated each probabilistic food-web as an ensemble of potential quantitative food-webs (where each link has a single link-strength), with link-strength probabilities determined by the mixing model. The patterns of species interactions within a community can be quantified from the statistical properties of the food-web. These statistical properties characterize the structural organization of interactions, and directly influence dynamics $[184,6]$. For example, when multiple consumers have overlapping diets, the food-web may be strongly nested [90]; when consumers have diets that are idiosyncratic, the food-web may be compartmentalized, or modular [91]. To quantify the structure of each probabilistic ensemble, both nestedness and modularity were calculated independently for a random draw of 1000 potential quantitative food-webs (see [205] for additional details).

Because predator-prey networks with nested or modular species interactions 
have strong dynamical implications, these properties are good measures of large-scale community-level organization. Nestedness $(\mathcal{N})$ quantifies the extent that specialist predator diets are subsets of generalist predator diets (calculated with the Nestedness based on Overlap and Decreasing Fill metric [7]). Nested trophic interactions can arise from groups of predators avoiding prey that fall below different optimal physiological or energetic requirements [107] or as a consequence of interspecific hierarchies between co-occurring predators [44]. By contrast, Modularity $(\mathcal{M})$, or compartmentalization (calculated from local link density [211]), is often observed in extant trophic systems $[67,184,15,205]$, and is thought to promote stability $[113,184]$ by isolating extinction cascades [179]. These structural metrics were assessed for each Pleistocene predatorprey network across cutoff values $i$. For detailed descriptions of structural metrics, see Appendix 3.2.

To examine how structural properties change as a function of link-strength, both nestedness and modularity were quantified across cutoff values $i$, such that a given property is first measured for the whole network $(i=0)$, and again at successive intervals as weak links are eliminated for higher cutoff values. The cutoff method works as follows: given a potential quantitative network within an ensemble, we binarized the link-strengths (see [10]). For the cutoff value $i$, a link exists if and only if it has a link-strength $>i$. The structural properties of each qualitative network were then analyzed for a set of cutoff values $i$ (i.e. $i=\{0,0.1, \ldots, 0.5\}$ ). For each measured structural property, this algorithm quantifies six values (one for each cutoff), describing how structural properties change as weak links are successively eliminated from the 
network.

Many structural properties correlate with food-web size [55]. To enable comparisons between food-webs with variable species richness, we measured nestedness and modularity as $\Delta \mathcal{N}_{i}$ and $\Delta \mathcal{M}_{i}$, respectively. As before, $i$ refers to the cutoff value, and $\Delta$ measures the difference between the structural measurement of an empirical (isotopic) food-web and a model network with 1) the same species richness, 2) the same predator:prey ratio, and 3) randomly drawn link-strengths. A value of '0' indicates no difference between the structure of the empirical food-web and that of the model; if $\Delta>0$, the empirical food-web has a higher value than expected by chance; if $\Delta<0$, the empirical food-web has a lower value than expected by chance.

\subsection{Results}

Estimates of trophic interactions between predator and prey groups differed across space and time. In the pre-Glacial, felids in both Beringia and Europe had relatively low proportional contributions of prey groups common to both regions (median contributions for 5 shared taxa, Beringia: 0.08; Europe: 0.07), with peak densities fairly symmetrical along the 1:1 line. By comparison, both Canis and Ursus arctos had peak densities that were strongly variable. We note that the posterior distribution of link-strengths between Canis and Caprine bovids were bimodal in Europe; bimodal link-strength distributions are interpreted as alternative hypotheses of predation, with probabilities reflected by the densities of prey contribution estimates. 
In the post-Glacial, felid prey-contribution peak densities were distributed along the 1:1 line, indicating that these species had similar diets over space. Unlike the pre-Glacial, where there was relatively even reliance on most prey species (Fig. 3.2 ), in the post-Glacial there was a significant contribution from Rangifer (median contribution, Beringia: 0.57; Europe: 0.51). Both Canis and Ursus show post-Glacial dietary contributions that differ from the pre-Glacial. Canids show a strong consumption of Bison in Beringia, but not in Europe (mean contribution, 0.57), whereas Ursus diets are focused on Rangifer in Beringia but not in Europe in the post-Glacial (median contribution, 0.66).

Consumer dietary specialization was calculated for predators in all mammoth steppe predator-prey networks (Fig 3.3). In Beringia, Arctodus was a specialist predator (particularly in the pre-Glacial; $\epsilon=0.58$; this and hereafter are median values), and relied primarily on either Rangifer or Symbos in the pre-Glacial, and switched to Bos in the full-Glacial $(\epsilon=0.35)$ (Fig. S1). Ursus, by contrast, was a generalist in the pre- and full-Glacial (pre: $\epsilon=0.22$; full: 0.23), but after the extinction of Arctodus, it switched to a specialized diet on Rangifer in the post-Glacial $(\epsilon=0.42)$. Canis and Beringian felids had generalist diets across the entire time interval (Canis pre: $\epsilon=0.24$, full: 0.23 , post: 0.32 ; felids pre: $\epsilon=0.22$, full: 0.23 ; post: 0.28 )

In Europe, more predators were specialists: Ursus (concentrating on Rupicapra; $\epsilon=0.36$ ) and Canis (concentrating on either Rupicapra or cervids; $\epsilon=0.48$ ) were specialists in the pre-Glacial, while Gulo, and Lynx had specialist diets in the postGlacial: scavenging (it is assumed) on Mammuthus $(\epsilon=0.55)$ and specializing on Lepus 
$(\epsilon=0.51)$, respectively (Fig. S3.2). By contrast, Crocuta had variable dietary proclivities in the pre-Glacial (ranging from generalism to specialism; Ardennes: $\epsilon=0.21$; Swabian Jura: $\epsilon=0.41$, while $H$. neanderthalensis was consistently a generalist consumer (Ardennes: $\epsilon=0.30$, Swabian Jura: $\epsilon=0.32$, based on a $\delta^{15} \mathrm{~N}$ discrimination factor of $4.5 \% 0$ ). When a $\delta^{15} \mathrm{~N}$ discrimination factor of $4.5 \%$ is applied (see Appendix 3.1), H. neanderthalensis was primarily consuming Mammuthus in the Ardennes ( $42 \%$ mean contribution) and both Mammuthus and Equus in Swabian Jura (41\% and 28\% mean contribution, respectively; Fig. S3.3), supporting results reported by Bocherens et al. [27]. An assessment of Neanderthal diet with a $\delta^{15} \mathrm{~N}$ discrimination factor of $3.5 \%$ increases estimates of Mammuthus specialization to $54 \%$ mean contribution in the Ardennes, and 73\% mean contribution in Swabian Jura (Fig. S3.3).

In Beringia, the across-predator reliance on specific prey showed strong similarities across the entire time interval (Fig. 3.4A). In the pre-Glacial, Bos, Symbos, and to a lesser extent Rangifer, were heavily preyed upon by the predator guild. After the local extinction of Symbos in the full-Glacial, Bos and Rangifer remained important prey resources, while the proportional contribution of Bison increased slightly. Across the interval, Equus, Mammuthus, and to a lesser extent Bison, had the lowest proportional contribution values. Dietary specialization of the predator guild changed slightly across the time interval (Fig. 3.4B), but increased in the post-Glacial $\left(\epsilon_{g}=0.35\right)$, indicating heavier reliance on a smaller subset of prey than in pre- and full-Glacial Beringia $\left(\epsilon_{g}=0.26\right.$ for both). Although European predators did not show consistent trends in prey reliance between pre- and post-Glacial, predator guild dietary specialization also 
increased in the post-Glacial period, from $\epsilon_{g}=0.34$ in both pre-Glacial Ardennes and Swabian Jura, to $\epsilon_{g}=0.44$ in post-Glacial Jura (Fig. S4).

Analysis of nestedness $(\Delta \mathcal{N})$ revealed that neither Beringian or European foodwebs were more nested than expected by chance (Fig. S3.5). Analysis of modularity $(\Delta \mathcal{M})$ revealed Beringian food-webs to be strongly modular in the pre-Glacial, particularly for cutoff values 0.2 to 0.5 (the strongest interacting species in the system) (Fig. 3.5A), no modularity in the LGM, and some modularity (cutoff values $0.2-0.3$ ) in the post-Glacial. By contrast, Europe showed little to no modularity across all cutoff values (Fig. 3.5B).

\subsection{Discussion}

The global cooling and drying trends associated with the LGM were particularly significant in northeastern Siberia and Beringia [68], but had large effects on the environment across the mammoth steppe. Analysis of the organization and magnitude of interactions between species in mammalian communities during these periods provides insight regarding 1) the extent to which species interactions varied across the mammoth steppe, 2) how interaction structures, measured on different scales, were impacted by the LGM, and 3) if so, whether these structures returned to a pre-perturbation state after the LGM. Understanding the flexibility of mammalian food-webs, and whether the interactions that form these systems can be re-established after global climatic perturbations, is relevant to current problems facing modern ecosystems. 


\subsubsection{Spatio-temporal patterns of species interaction}

Our comparison of Beringian and European link-strength distributions show felid diets to be more constrained over space than those of Canis or Ursus, particularly in the post-Glacial (Fig. 3.2). Rangifer became an important component of felid diets in the post-Glacial, coinciding with an observed increase in Rangifer abundance, particularly in North America, ca. 20 kyr BP [99], although we cannot rule out that this dietary switch could be the consequence of behavioral alterations independent of prey population dynamics, The peak densities of prey contribution to diet for Canis and Ursus show different patterns than those of felids; however the increase in Rangifer abundance may have impacted these predators as well. The dissimilarity between Canis and Ursus diets over both space and time highlights their ecological plasticity, particularly during the post-Glacial. Although previous studies have shown Canis to be a generalist predator during the Pleistocene [95, 59], we show that their reliance on prey species is both general and highly flexible. Modern wolves are opportunistic predators [133, 174], but often specialize on locally abundant cervids [120]. The variability of Pleistocene canid diets may be due, in part, to a higher diversity of wolf morphs during the late Pleistocene, with the Beringian population noted for a cranial morphology associated with scavenging [95]. Although the intercontinental ranges shared by felids, Canis, and Ursus, are a testament to their success, felids appear to have more constrained diets over the mammoth steppe ecosystem. If dietary constraints are riskier to maintain over long time-scales $[116,193,151]$, such differences between taxa may have contributed to 
the extinction of large felids from the mammoth-steppe region, while Canis and Ursus remained abundant into the late Holocene.

While felids showed consistent dietary trends across the mammoth steppe, particularly in the post-Glacial, our quantification of dietary specialization indicates that they were strong dietary generalists, particularly in Europe (Fig. 3.3). Similarly, Canis and Ursus were generalist feeders, with some temporal variation. By contrast, the shortfaced bear Arctodus was a dietary specialist in the pre-Glacial, primarily concentrating on Rangifer (supporting results by Fox-Dobbs et al. [59]). However, in the full-Glacial, Arctodus prey-contribution estimates indicate a dietary switch towards Bos, after which the short-faced bear disappears from the paleontological record. It is interesting to note that Arctodus is the only Beringian predator whose reliance on Rangifer decreased after the pre-Glacial. Rangifer abundance increased after the pre-Glacial in Beringia, and many predators altered their diets to include a higher proportion of this prey. If Rangifer was a preferred food of Arctodus (as the pre-Glacial isotope record suggests), a scenario in which short-faced bears were competitively displaced, or forced to alter their diets, by co-occurring predators is a possibility.

In Europe, predator specialization appears to be more variable. Canis is alternatively a moderate specialist and strong generalist in the pre- and post-Glacial, respectively. The European hyena Crocuta is highly variable, bearing a strong generalist signal in the Ardennes (with some concentration on Mammuthus), while individuals specialized on both Mammuthus and Equus in Swabian Jura (both pre-Glacial sites). The low felid $\epsilon$ values in the pre-Glacial (possibly due to dietary specialization among 
individuals [28]) are similar to those for neanderthals, however prey-contribution results show felids to rely on Rangifer (particularly in Swabian Jura), while neanderthals consumed primarily Mammuthus and Equus. We have not considered the impact of Homo sapiens in European sites, and cannot rule out the possibility that the presence of human hunter-gatherers may have contributed to the observed variance in predator specialization.

\subsubsection{Spatio-temporal patterns of community organization}

Patterns in resource acquisition are strongly consistent in pre-, full-, and postGlacial Beringia (Fig. 3.4A). Although it has been noted that Mammuthus was underutilized in Beringia [59], our results show a similarly low reliance on Bison and Equus. We found strong predator reliance on Bos throughout the LGM, and a shift from Symbos in the pre-Glacial to Rangifer in the full- and post-Glacial. The low proportional contribution of Bison may be the consequence of a sharp decline in Bison abundance beginning ca. $35 \mathrm{kyr} \mathrm{BP}$, and accelerating after $16 \mathrm{kyr} \mathrm{BP}[164,99]$. A shift to a reliance on Rangifer by the predator guild mirrors the dietary switch observed for felids. There are no consistent patterns in resource acquisition among European predators (Fig. S3.4), however, in contrast to Beringia, Mammuthus is a more important prey resource across the entire time interval, while Equus is an important resource in all sites but the Ardennes.

We find in both Beringia (Fig. 3.4B) and Europe (Fig. S3.4) that predator guild specialization $\left(\epsilon_{g}\right)$ increased in the post-Glacial, indicating that a smaller pro- 
portion of available prey was heavily used across predator species. Declines in species richness, the biomass of available prey, or changes in prey abundance undoubtedly affected predator species differently. The observed increase in predator guild dietary specialization indicates a general trend towards increasing resource specialization among predators. Similar trends have been observed for extant consumers during periods of dietary stress $[160,185]$. In fact, increasing values of $\epsilon_{g}$ across the study interval is coincident with the general decline and range contraction of many Eurasian herbivores [99]. We note that the early arrival of neanderthals and later, humans (earliest dates: ca. 40-45 kyr BP [119]), to Europe, may have accelerated such processes if these species strongly contributed to food limitation.

Our analysis of nestedness and modularity $(\Delta \mathcal{N}$ and $\Delta \mathcal{M}$, respectively) reveal within-region similarities and between-region differences from pre- to post-Glacial. Dynamical analyses have shown nestedness to be a destabilizing structure in food-webs $[184,6]$. The primary source of modularity in pre-Glacial Beringia originates from a strong similarity in prey choice by Canis and Ursus, whereas felids and Arctodus have more idiosyncratic diets across the LGM (Fig. S3.1). These large-scale structural differences highlight potential variability in the organization of species interactions, despite similar taxa, across the mammoth steppe.

\subsubsection{Linking species interactions to large-scale community structure}

Modularity is associated with dynamic stability and increased persistence [67, 184, 179]. High relative modularity $(\Delta \mathcal{M}>0)$ implies that the pre-, and perhaps the 
post-Glacial Beringian systems are more internally stable across the LGM relative to European systems. There are two potential explanations that account for differences in modularity between Beringia and Europe. The spatial distribution of either plant species with physiognomic similarities or micro-habitats could contribute to the isotopic ratios of herbivores that result in modular predator-prey networks. Modern East African food-webs are organized in spatial guilds that contribute to modular structures [15], particularly among strongly linked species [205]. In Beringia, spatial variability of plant isotope ratios could arise from 1) isotopic differences in plants inhabiting different microhabitats (where small differences in humidity, rainfall, or soil moisture may impact the isotopic values of local plant tissues [134]), leading to differences among herbivores that consume plants in these micro-habitats (and by extension, their predators), or 2) isotopic differences among different plant functional types (e.g. shrubs, grasses, lichen), such that the dietary preferences of herbivores result in isotopic differentiation among browsers, grazers, and their respective consumers [21, 52]. Two lines of evidence suggest that the latter is more likely: within-region variation in herbivore dental micro- and mesowear reveal strong dietary differences among herbivores [155], and significant differentiation exists in the isotopic values of different plant functional groups [59]. Thus, we conclude that herbivores accumulated unique isotopic values as a function of dietary differences rather than foraging in different, isotopically-distinct micro-habitats. Although the spatial patterning of vegetation in Beringia is disputed [68, 209], there is little support for spatial differentiation of plant functional types $\mathrm{n}$ at the scale present in African savanna-woodland environments, particularly during the full-Glacial [68], suggesting 
that their may be an alternative explanation for Beringian network modularity.

An alternative explanation of modular network structure may involve the insularity of the Beringian mammoth-steppe community. Modular food-webs are defined by dietary resource segregation among consumers [67]. Resource segregation can occur over ecological, but also evolutionary timescales, where coevolutionary relationships may begin to constrain the plasticity of trophic interactions, promoting compartmentalization [65]. In isolated environments, where neighboring systems are similar and invasions are rare, differentiation of resources and the subsequent development of modular interactions may be more likely to occur and be reinforced over time. In contrast, systems that are bordered by a diverse array of animal communities and are highly diffuse may be held in a transient state such that niche diversification is continually interrupted, limiting compartmentalization. We suggest that pre-Glacial Beringia may have been modular due to stronger homogeneity with, and periodic isolation from, neighboring communities. This insularity would serve to limit invasions of species from dissimilar communities, allowing consumers to minimize competitive overlap while maximizing resource diversity. Europe, by comparison, was an ecological nexus [2], where the periodic influx of species from diverse communities may have limited niche diversification among species, preventing compartmentalization.

If Beringian modularity was a consequence of insularity, we would expect an external perturbation to disrupt this structural feature. The full-Glacial was characterized by abrupt changes in herbivore abundances [99] and large-scale climate change that significantly altered the distribution of mammoth steppe vegetation $[68,208,35]$. As 
would be expected under this scenario, Beringian predator-prey networks show no evidence of modular structures during the full-Glacial (Fig 3.5). Moreover, there is a hint that modular patterns of interaction may have emerged during the post-Glacia, and the application of a trophic discrimination factor closer to $4.6 \%$ results in $\Delta \mathcal{M}$ values that are significantly greater than zero (Fig. S3.6). It is tempting to speculate that modularity may be recoverable after a large perturbation to community structure, particularly if the system is insulated from additional disturbances. We are not aware of any experiment that tests this specific mechanism for preserving (or disrupting) modularity, and we suggest that this would be a fruitful theoretical exercise.

Modern mammalian communities are remnants of a rich Pleistocene heritage. Understanding the relationships between Pleistocene species will inform understanding of extant ecosystems. Moreover, studies of past ecosystems permit an examination of how communities responded to climatic or other external perturbations over long timescales. Because some species inhabiting mammoth steppe environments are present (and in many cases at risk) in modern ecosystems, reconstruction of species interaction structure across the LGM is increasingly relevant for understanding the potential resilience and plasticity of modern species interactions.

\section{Acknowledgements}

We are grateful to S Allesina, T Gross, AM Kilpatrick, C Kuehn, T Levi, M Mangel, M Novak, M Pires, PI Prado, L Rudolf, AO Shelton, DB Stouffer, and C 
Wilmers for helpful discussions and/or criticisms. The Ecological School of Networks Workshop in São Pedro, SP Brasil provided a singular opportunity to discuss many of the ideas that encouraged and improved this work. This research was funded from a National Science Foundation (NSF) Graduate Research Fellowship and a UC-Santa Cruz Chancellors fellowship to JDY. 


\subsection{Figures}

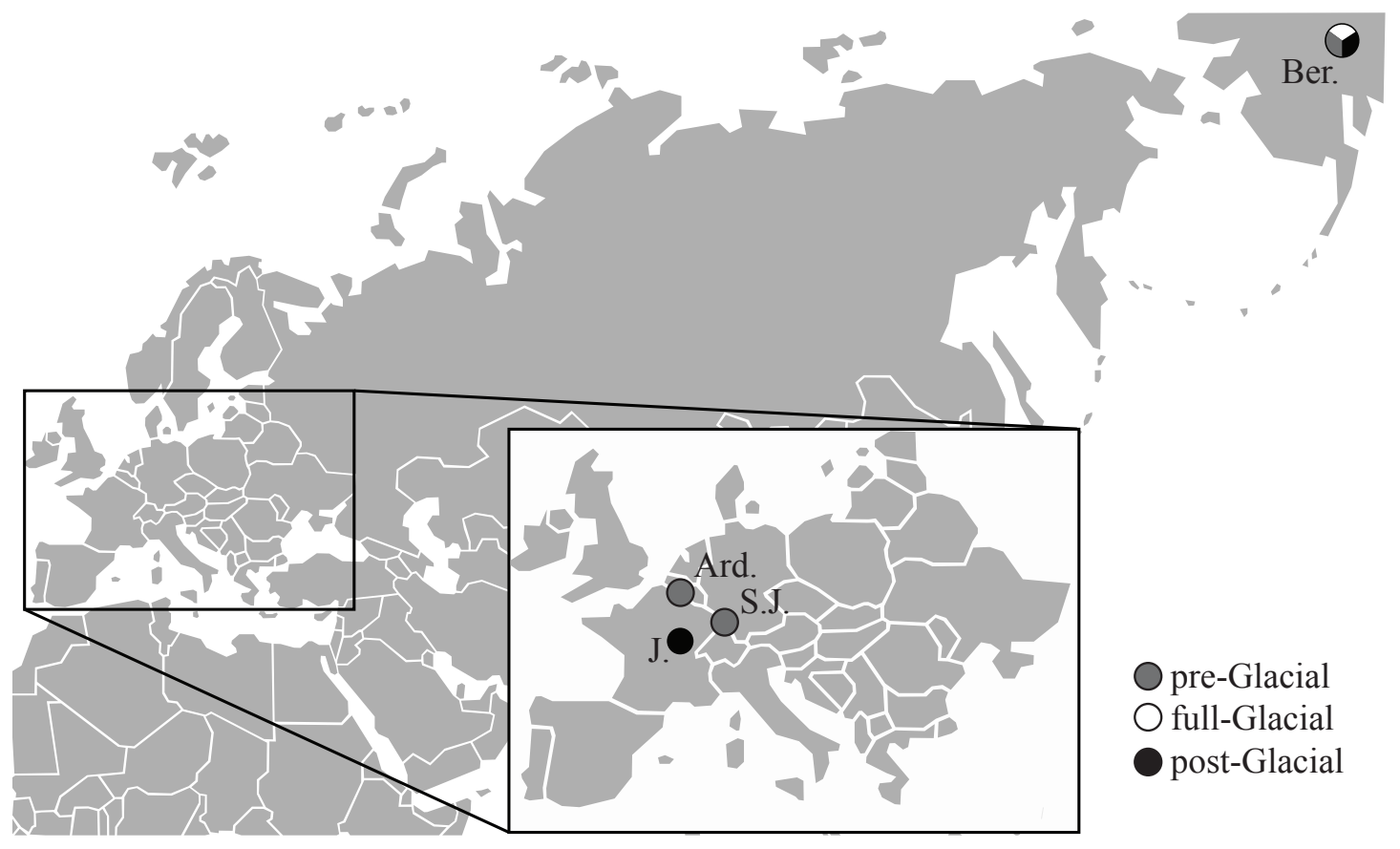

Figure 3.1: Locations of late Pleistocene mammoth steppe sites included in the analysis. The pre-, full-, and post-Glacial Beringian sites are located near Fairbanks, Alaska. Two pre- and one post-Glacial European site occur in eastern France, Belgium, and western Germany, respectively. Ber. = Beringia; Ard. = Ardennes; S.J. = Swabian Jura; J. = Jura. 


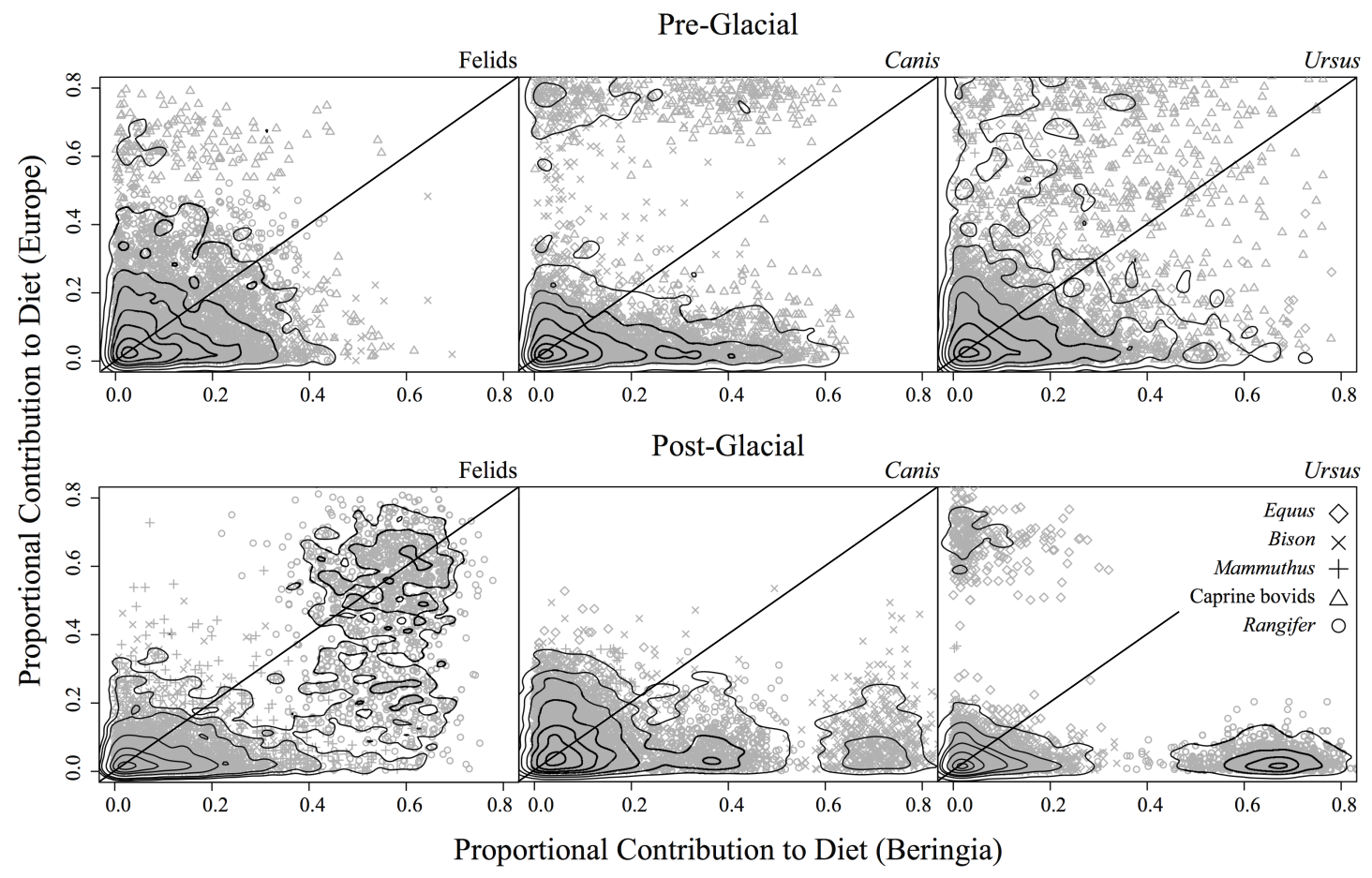

Figure 3.2: Proportional contribution estimates of prey taxonomic groups to the diets of predator groups present in both Beringia and Europe in the pre- and post-Glacial periods (grey symbols). The contribution estimates represent numerical approximations of link-strength posterior probability distributions from the Bayesian isotope mixing model, MixSIR. Densities of contribution estimates across all prey were calculated with a kernel density algorithm [56]. 


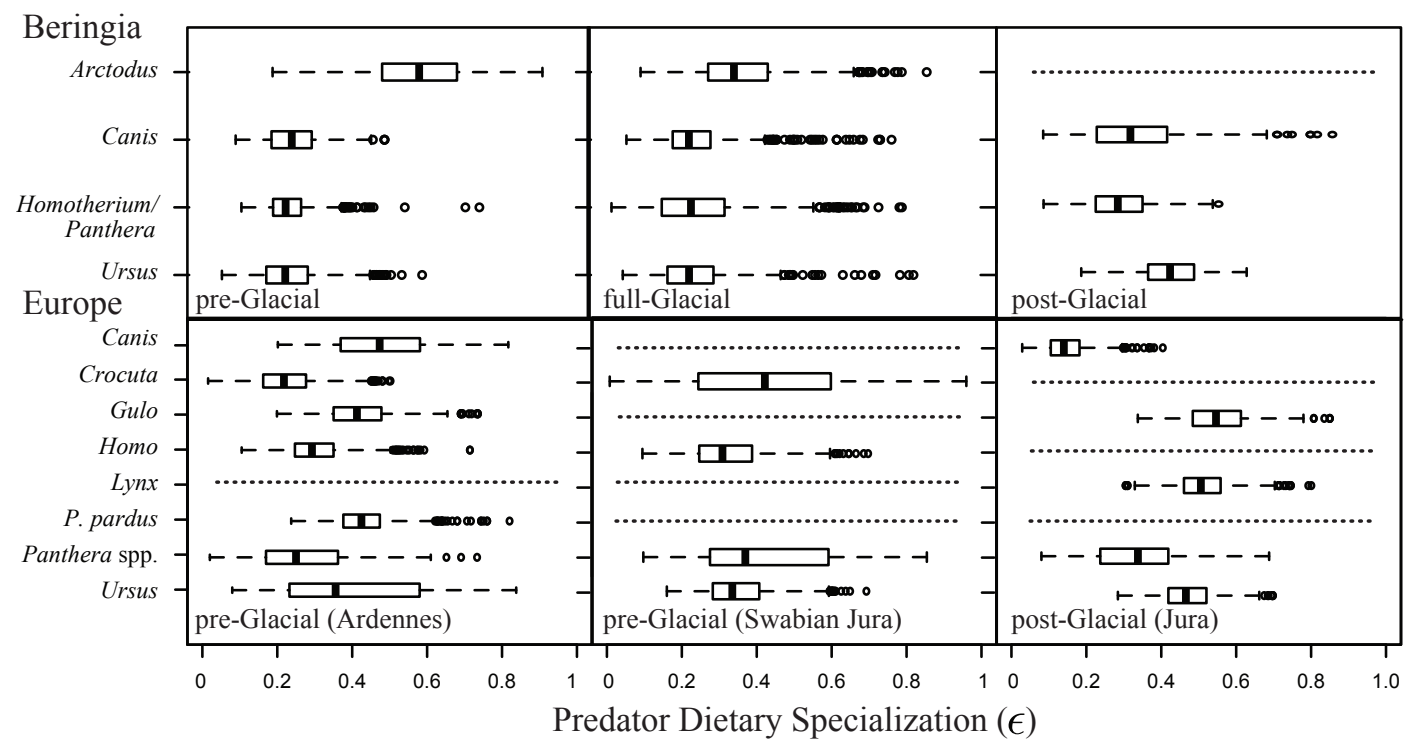

Figure 3.3: Predator dietary specialization $(\epsilon)$ for Beringian and European predator guilds from the pre-Glacial to the post-Glacial. A value of $\epsilon=0$ describes a generalist diet (consumption of all prey in equal amounts), whereas a value of $\epsilon=1$ describes a specialist diet (consumption of one prey to the exclusion of others). Dotted lines denote species' absence. 
A. Across-Predator Proportional Contribution to Diet

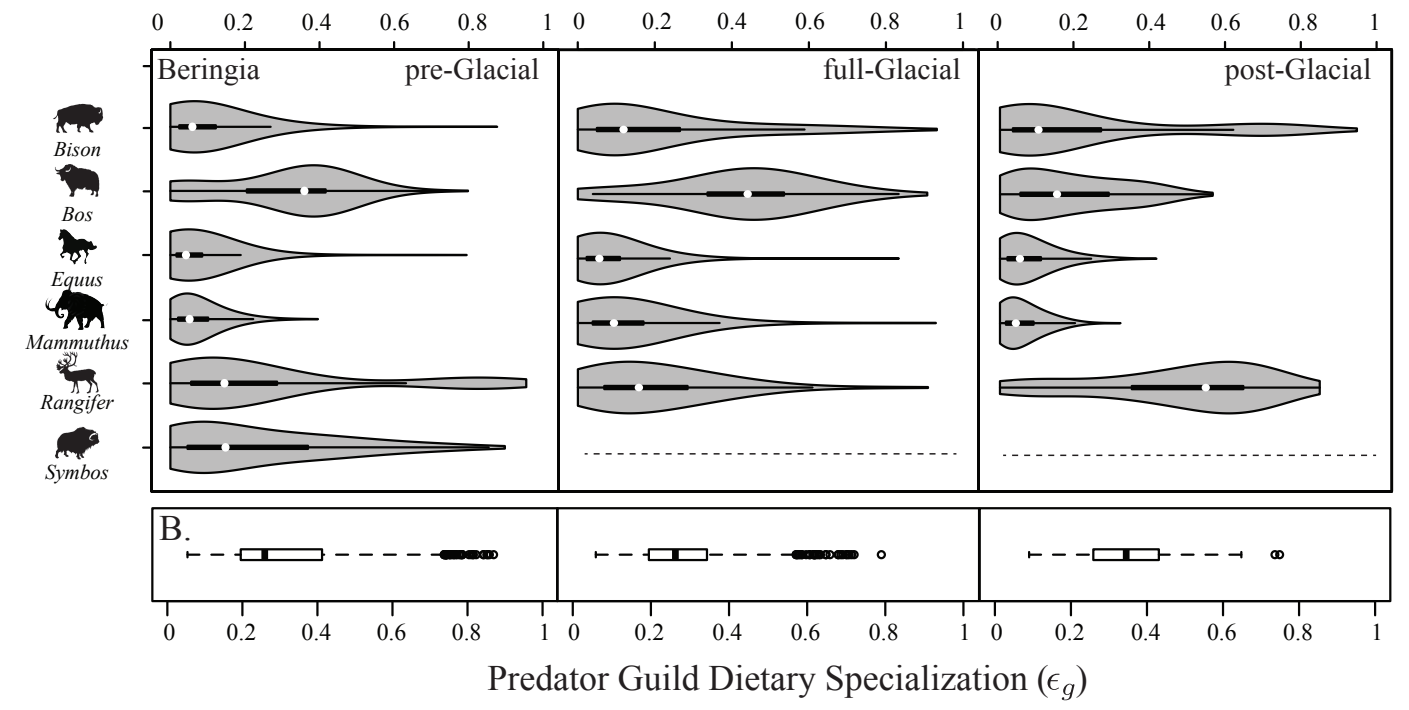

Figure 3.4: A. The proportional contribution of each prey with respect to all potential predators in Beringia in the pre-Glacial, full-Glacial, and post-Glacial. High values denote prey with higher proportional contributions across predators, whereas low values denote prey with lower proportional contributions. B. Consumer dietary specialization quantified for the predator guild $\left(\epsilon_{g}\right)$ for each time period. The median $\epsilon_{g}$ value is highest for the post-Glacial, indicating an on-average greater contribution of a smaller subset of potential prey; this trend is also observed in European systems (Fig. S3.4). Dotted lines denote species' absence. 


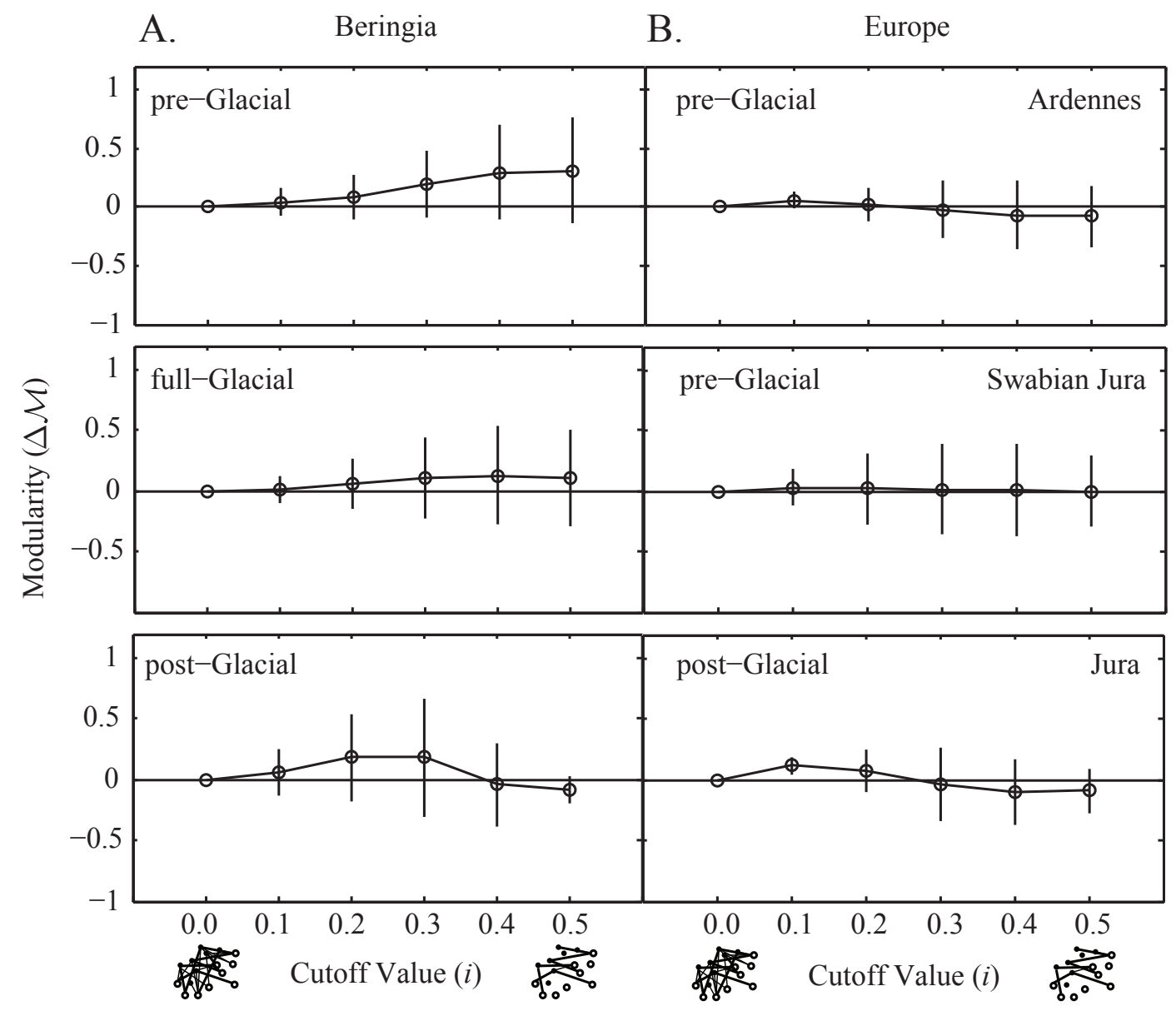

Figure 3.5: Modularity $(\Delta \mathcal{M})$ for A. Beringia and B. Europe across cutoff values $i$. Values $>0$ indicate that the system is more modular than would be expected given the size and predator:prey ratio of the food-web; values $<0$ indicate that the system is less modular than would be expected. The cutoff value $i=0$ refers to the whole food-web with no link deletions; cutoff values 0.1 to 0.5 describe the food-web when links $\leq 0.1$ to 0.5 are eliminated, respectively. At high cutoff values only the strongest interacting species affect the structure of the food-web. 


\subsection{Supplementary Material}

\subsubsection{Appendix 3.1. Link-strength posterior probability distributions}

Stable isotope ratios can be used to estimate the proportional contribution of prey to a predator's diet with Bayesian isotope mixing models. The proportional contribution of prey to a given predator's diet is calculated numerically, and is represented by a single multivariate posterior distribution. The numerical estimation of the posterior distribution can be visualized as a matrix, where each column represents the proportional contribution of a single prey, and each row is a potential combination of link-strengths, which is constrained to sum to one. As such, a food-web consists of a single matrix for each consumer in the system.

To account for individuality within consumer species, particularly for cases where the mean isotope values of a predator are not descriptive of the population, we 1) quantified a posterior distribution for each individual of each predator species, and 2) sampled with equal weight from the individual posterior distributions to quantify a species-level distribution. This method operates under the assumption that a species' reliance on prey is a direct composite of the diets quantified for each individual; if a particular diet is represented by a large proportion of individuals, the species-level posterior distribution will be biased towards this dietary strategy. We used the specieslevel distribution for all subsequent food-web analyses. 


\subsubsection{Appendix 3.2. Structural metrics}

We analyzed the nestedness $\left(\mathcal{N}_{i}\right)$ and modularity $\left(\mathcal{M}_{i}\right)$ of three European and three Beringian food-webs that spanned the Last Glacial Maximum (LGM). The subscript $i$ denotes the cutoff value at which structure is being measured. For example, a cutoff value $i=0.1$ means that a given property is being measured for a food-web where all links with link-strength $\leq 0.1$ have been eliminated; higher cutoff values denote structural properties measured for the strongest links in the food-web. Both structural properties were compared against nestedness and modularity values for model networks to control for correlations between structure and both food-web size (species richness) and predator:prey ratios (see text). The values of $\mathcal{N}_{i}$ and $\mathcal{M}_{i}$ are thus reported as $\Delta \mathcal{N}_{i}$

and $\Delta \mathcal{M}_{i}$, where $\Delta$ denotes the difference between the empirical (with link-strengths drawn from the posterior distributions of mixing model results) and model food-web structure property (with link-strengths drawn at random). A value of ' 0 ' indicates no difference between the structure of the empirical food-web and that of the model; if $\Delta>0$, the empirical food-web has a higher value than expected by chance; if $\Delta<0$, the empirical food-web has a lower value than expected by chance.

We measured nestedness, $\mathcal{N}_{i}$, with the commonly-used Nestedness based on Overlap and Decreasing Fill (NODF) metric [7], and scaled values such that they varied between 0 and 1 rather than 0 and 100. Accordingly, a value of 0 describes an non-nested food-web, while a value of 1 describes a completely nested food-web, were the interactions between a specialist predator and its prey are subsets of interactions 
between generalist predators and their prey.

We measured modularity, $\mathcal{M}_{i}$, by quantifying the density of network 'squares', which are defined as a subset of two predators completely connected to (interacting with) two prey within a food-web; in terms of nodes and links, a square occurs when four nodes are connected by at least four links. Accordingly, the average density of squares $\left(\bar{\rho}_{s}\right)$ equals the number of squares in a network divided by the number of potential squares in a completely connected network, i.e. the local link density. This metric has the advantage of being analytically tractable for any network size, whereas other metrics use optimization methods [66]. As the connectance, $C$, of a network increases $(C=l / m n$, where $l$ is the number of links in a network, and $m$ and $n$ are the number of prey and predator species, respectively), the local link density increases. We calculated modularity as

$$
\mathcal{M}=\frac{\bar{\rho}_{s}-C}{\bar{\rho}_{s}+C}
$$

If the average local link density becomes greater than the overall link density, $\mathcal{M} \rightarrow+1$; if the average local link density becomes less than the overall link density, $\mathcal{M} \rightarrow-1$ $[10]$ 


\subsubsection{Figures}
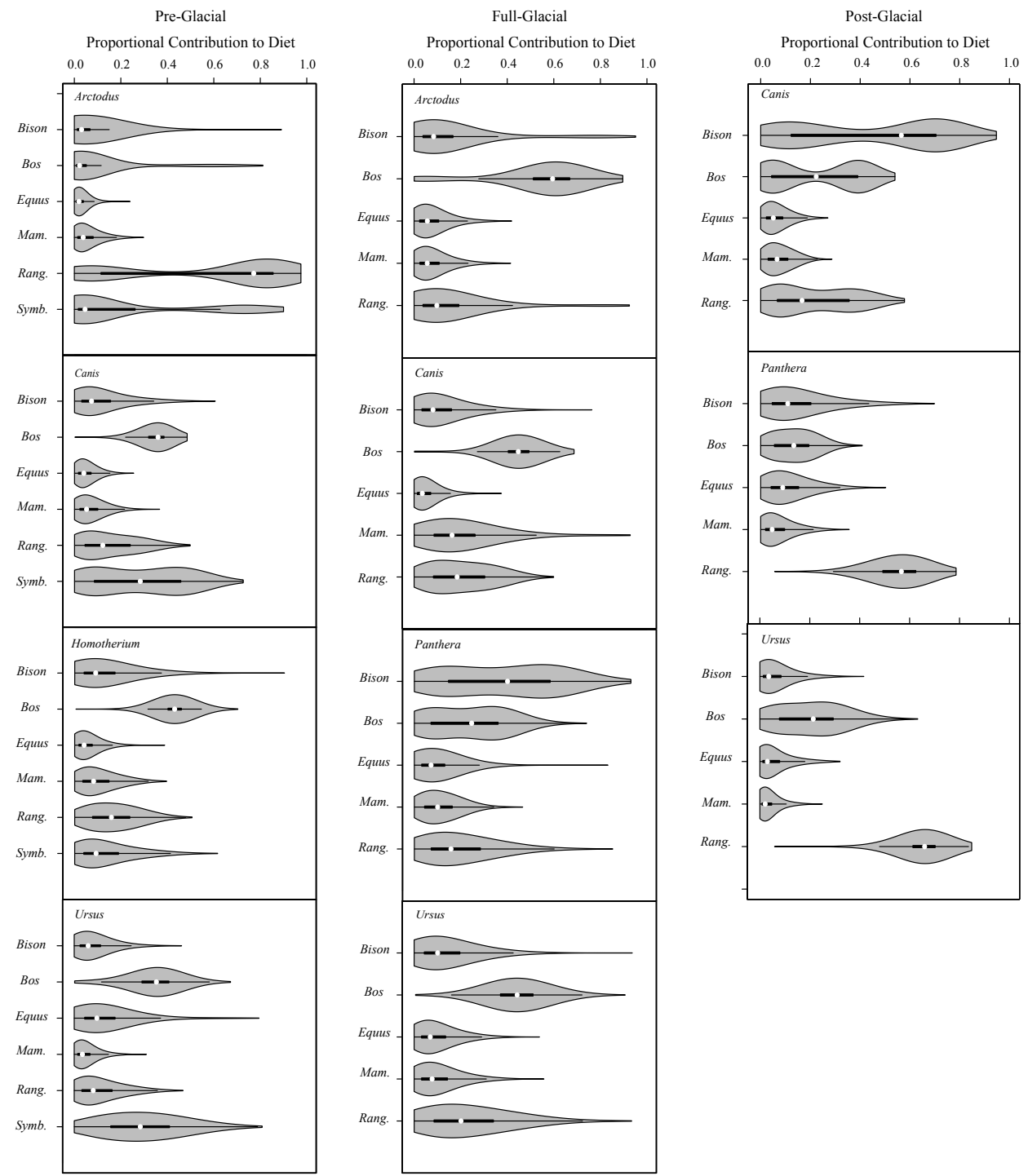

Supplementary Figure 3.1: Proportional contribution of prey to predator species in pre-, full-, and post-Glacial Beringia. Boxplots denoting the 25\%,50\%, and $75 \%$ quartiles are depicted by the black bars (the white point denotes the median value), and the underlaying distribution is depicted in grey. Bimodality is interpreted as alternative hypotheses of predator diet, where the probability of each hypothesis is determined by the density of the distribution. 

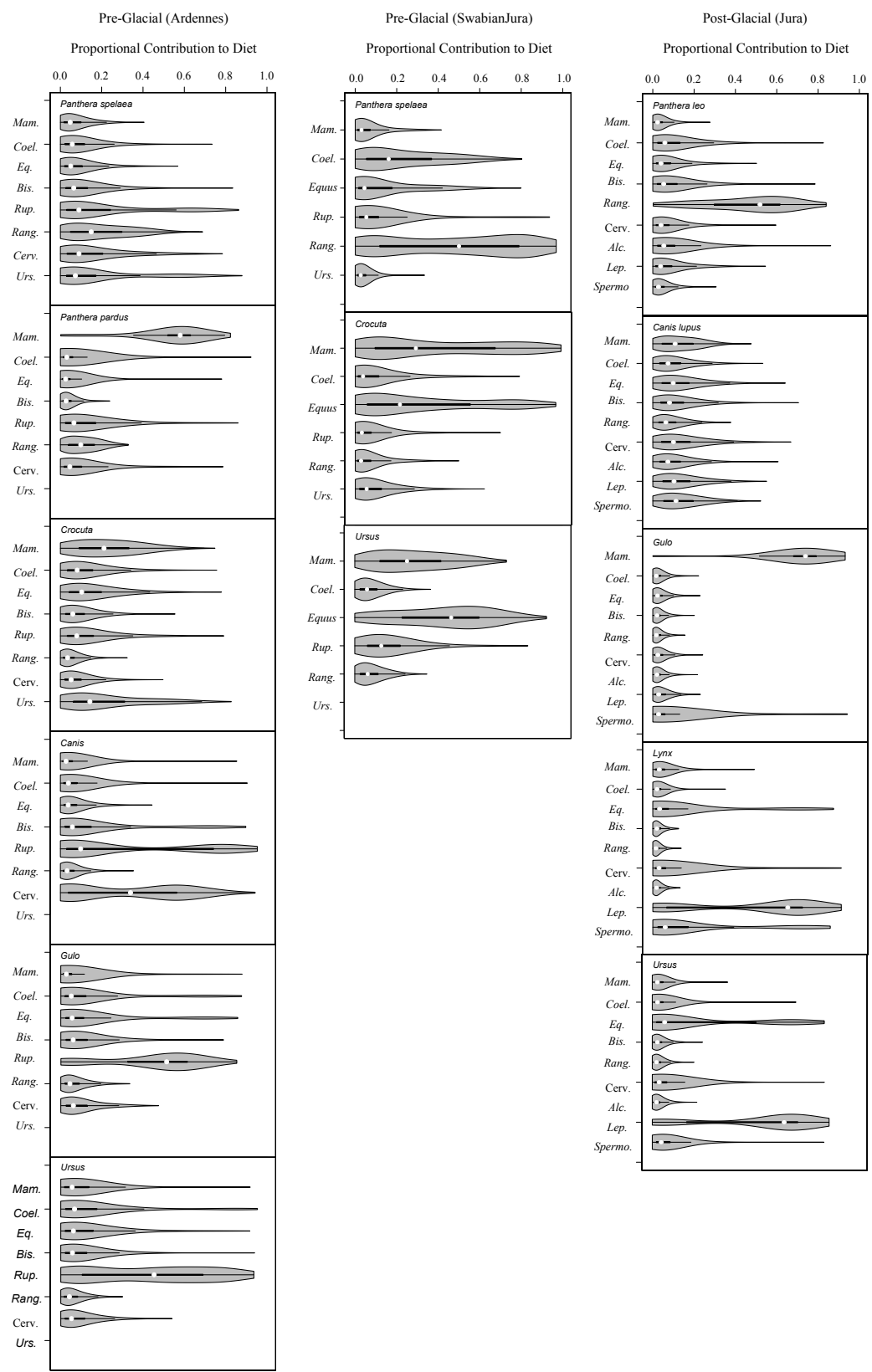

Supplementary Figure 3.2: Proportional contribution of prey to predator species in two pre-Glacial and one post-Glacial site in Europe. 


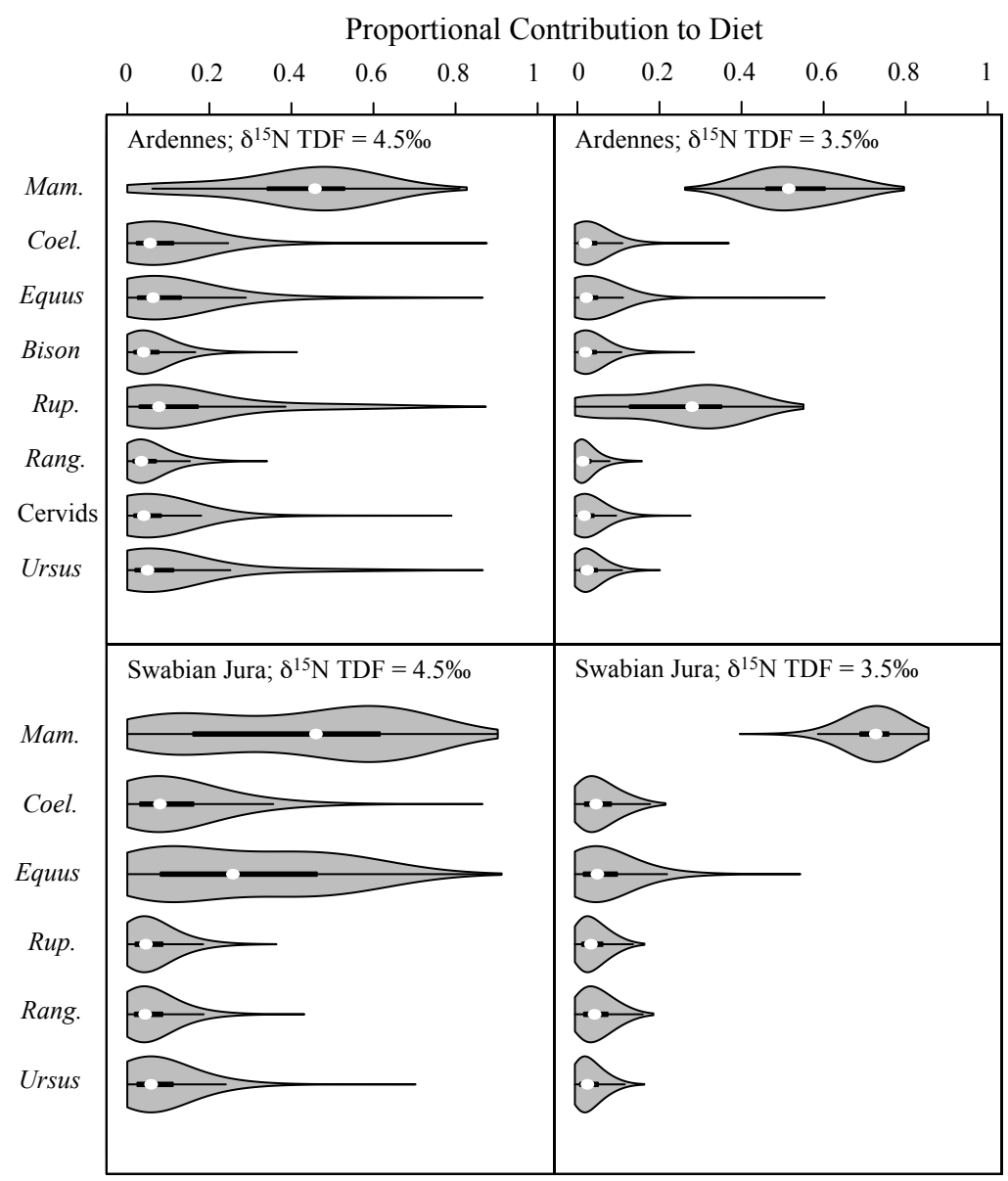

Supplementary Figure 3.3: The proportional contribution of prey to Neanderthal diets for the two pre-Glacial European sites: Ardennes and Swabian Jura. Two alternative dietary estimates are presented: one when a trophic discrimination factor (TDF) of $4.6 \%$ (low quality protein diet) is applied, and one when a TDF of 3.5\% (high quality protein diet) is applied. 


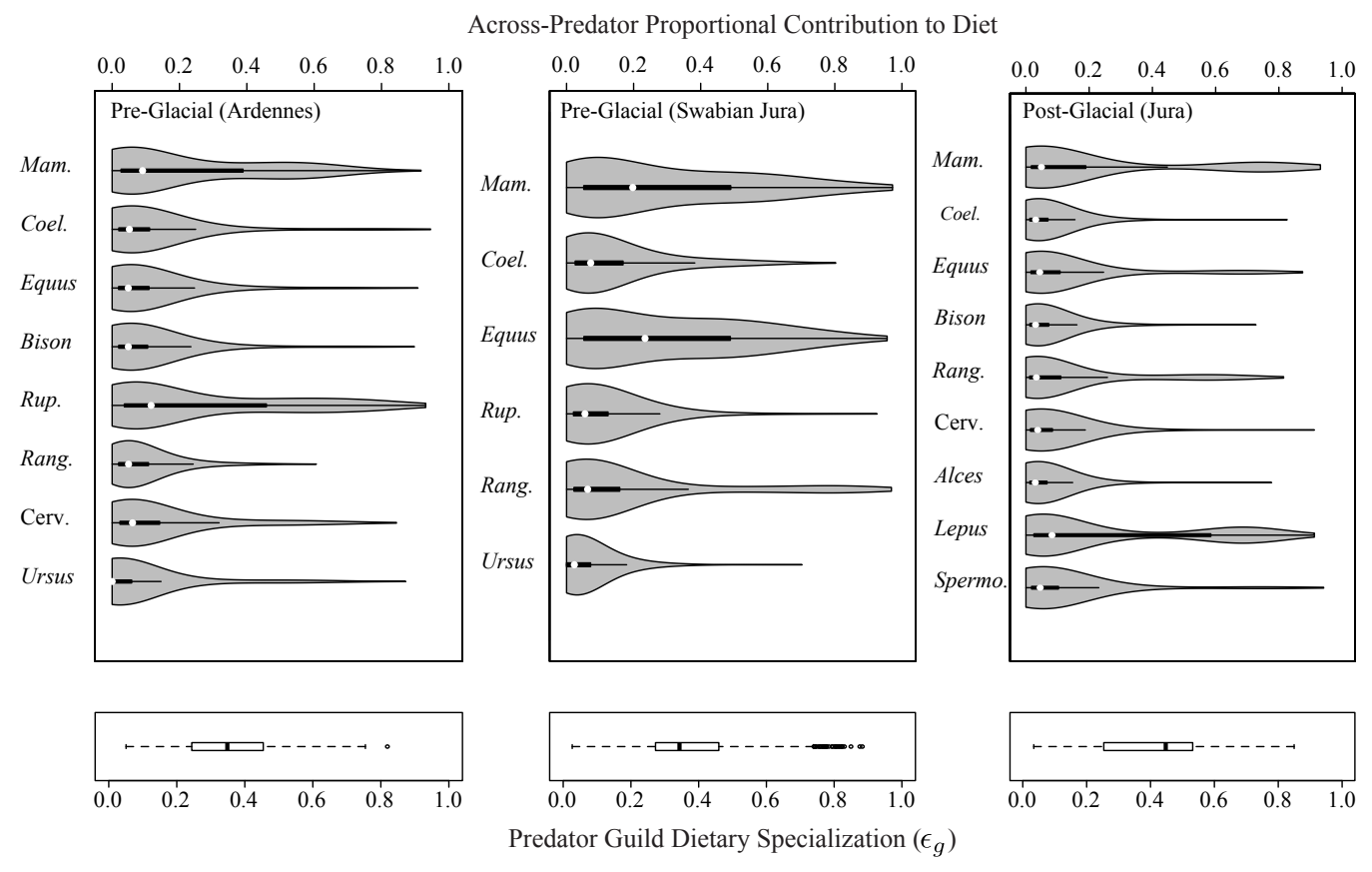

Supplementary Figure 3.4: A. The proportional contribution of each prey with respect to all potential predators in Europe across the LGM. High values denote prey with higher proportional contributions across predators, whereas low values denote prey with lower proportional contributions. B. Consumer dietary specialization quantified for the predator guild $\left(\epsilon_{g}\right)$ across the LGM. The median $\epsilon_{g}$ value is highest for the post-Glacial, indicating an on-average greater contribution of a smaller subset of potential prey. 

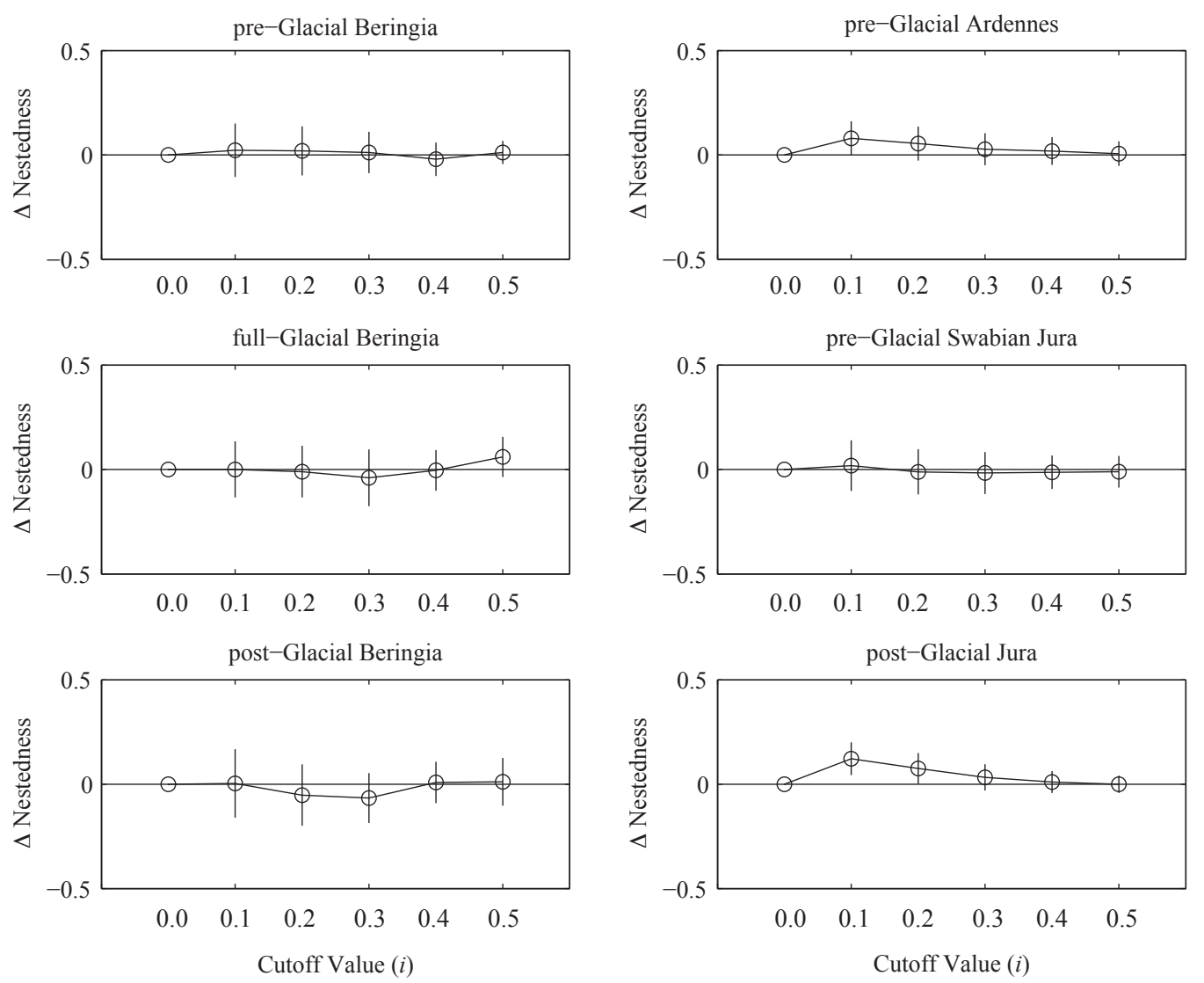

Supplementary Figure 3.5: Nestedness $(\Delta \mathcal{N})$ for A. Beringia and B. Europe across cutoff values $i$. Values $>0$ indicate that the system is more modular than would be expected given the size and predator:prey ratio of the food-web; values $<0$ indicate that the system is less modular than would be expected. The cutoff value $i=0$ refers to the whole food-web with no link deletions; cutoff values 0.1 to 0.5 describe the food-web when links $\leq 0.1$ to 0.5 are eliminated, respectively. At high cutoff values only the strongest interacting species affect the structure of the food-web. 


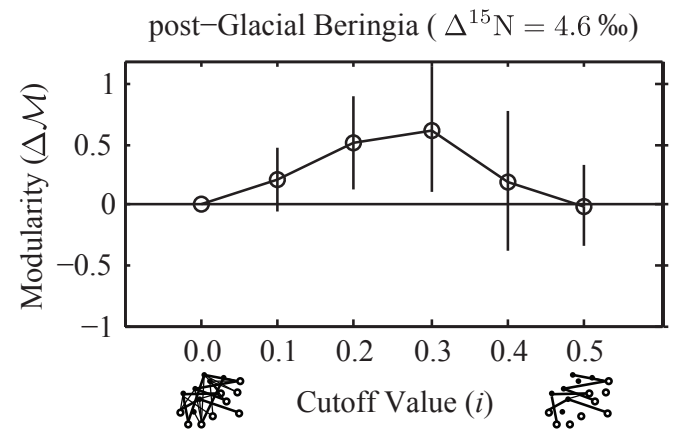

Supplementary Figure 3.6: $\Delta$ Modularity for the post-Glacial Beringian food-web with a trophic discrimination factor of $\Delta^{15} \mathrm{~N}=4.6 \%$ applied. 
Chapter 4

Foraging behavior and the constraints of

enamel: Using a process-based model to

predict the dietary benefits of

mechanical advantage and megadont

dentition among hominins

J D Yeakel, N J Dominy, P L Koch, M Mangel 


\section{Abstract}

Foraging behavior is limited by the energetic demands of resources, but must also balance additional mechanical costs that are not readily apparent. Such mechanical costs may contribute to wear on instruments used to procure or consume resources, thus constraining the roles of some foods. Molar enamel thickness is one of the primary morphological traits that differentiate anthropoid primates in general and hominin species in particular - being most accentuated among megadont hominins (Paranthropus spp.) - and correlates strongly with diet. Among living primates, molar enamel thickness is directly related to feeding on fracture resistant foods. Plant Underground Storage Organs (USOs) in particular are thought to be important resources for early hominins, in part due to their accessibility and mechanical characteristics. However the relationships between foraging behavior and enamel wear have not been explored. Here we present a process-based model to investigate foraging constraints as a function of energetic demands and enamel wear among anthropoid primates. Our framework allows us to explore under what conditions foods such as USOs are chosen as fallback, rather than preferred, resources. Moreover, we quantify the potential fitness benefits of behavioral and evolutionary adaptations to consume fracture resistant foods, whether by modifying the mechanical properties of food or employing megadont dentition. Finally, our model predictions help resolve the noted disparity between hominin isotopic and microwear dietary evidence, and we show how this approach can be used not only to predict, but to update paleo-dietary information using Bayesian approaches. 


\subsection{Introduction}

In 1992, F. Juanes [80] observed decapod crustaceans to select smaller prey than predicted by classical foraging theory, and suggested that feeding-induced wear on claw teeth was responsible for this difference. In fact, many consumers have physiological resources used to obtain food that are either nonrenewable, or that renew slowly [81]. Over the course of an organism's lifetime, mechanical wear on these resources must be minimized, while the net energy obtained from food, or in some cases invested in reproduction, is maximized. These physiological resources vary across organisms, including oystercatcher beaks [182], wasp ovipositors [79], the mandibles of plant-eating insects [158], and the molar enamel of mammals [177, 114, 167, 143], including modern and extinct hominins $[112,170,191]$.

Dental enamel is a hard, mineralized tissue covering the elastic and vascularized dentin, and rooted by cementum to the jaws of most mammals [104]. Comminution of food is a uniquely mammalian trait among modern species (in addition to some ornithischian dinosaurs; [197]), and serves to reduce food particle size prior to digestion [104]. Although some animals have renewable dental resources, primates are dyphodont, meaning their teeth are replaced only once, after which the dentition is nonrenewable. Because enamel is nonrenewable, and prone to damage [45], consumption must be balanced with preservation. Accordingly, the evaluation of a food's mechanical characteristics prior to consumption is a critical foraging decision.

Molar enamel thickness is phylogenetically conserved, and animals with thicker 
molar enamel have diets that include mechanically hard, fracture resistant, foods [102]. Although enamel thickness is allometrically constrained, both modern and extinct savannawoodland primates display a larger range of enamel thickness than expected from body size [165]. Of these species, both savanna chimpanzees (Pan) and baboons (Papio), and the earliest extinct hominins (Sahelanthropus and Ardipithecus) tend to have thinner molar enamel, while Theropithecus, Homo, and some derived hominins (Australopithecus) tend to have thicker molar enamel $[165,105]$. At the extreme, Paranthropus spp. were megadont hominins with unusually thickly enameled molars [105]. Isotopic evidence shows these animals to have diets influenced by $\mathrm{C}_{4}$-photosynthetic plants (e.g. tropical grasses or sedges - or potentially animals that consumed these plants) $[175,191]$, which tend to be fracture resistant, however patterns of molar microwear indicate a diet of softer, less fracture resistant foods [189, 190]. Importantly, the isotopic composition of molar enamel forms early in life whereas patterns of microwear are recorded later in life, such that the potential influence of life-history stages cannot be ignored. Although life-history differences may contribute to this long-standing paradox, the same inconsistencies may also be due to fracture resistant foods (such as plant Underground Storage Organs - USOs) serving as fallback rather than preferred dietary resources [181]. The physiological and behavioral processes that may have contributed to these opposing patterns have not been explored or quantified.

Here we present a Stochastic Dynamic Programming (SDP) model [109, 41] to quantify the optimal foraging decisions for organisms that balance energetic gain with enamel wear, in addition to the stochastic effects of a variable environment. We 
establish our model on measurements for anthropoid primates and focus specifically on decisions affecting hominins in savanna-woodland environments. Our goal is to develop a process-based model, where an optimal foraging decision is specified for all internal states an organism experiences, and across a set of stochastic environmental conditions. Accordingly, decision matrices are constructed whereupon specific food resources are determined to maximize an animal's fitness, conditional on two internal states: the organism's energetic reserves and molar enamel volume.

This model-based approach is well-suited to test a variety of important questions regarding the effects of dental enamel on foraging, and we focus on three potentially informative lines of inquiry. First, and most essential, how is the quantity of enamel predicted to influence the foraging strategies of savanna-woodland anthropoid primates, and how does megadont dentition alter these strategies? Second, to what degree do these foraging decisions dependent on resource quality and quantity, where the quality and quantity of particular food items may vary with habitat? Third, can specific mechanical advantages, such as peeling or pounding/cooking alter decision matrices, and to what extent do these alterations provide fitness benefits? Finally, we relate our model predictions to paleontological evidence of hominin diets, and conclude by showing that the model framework presented here can be used to not only predict, but update paleodietary data. 


\subsection{Methods}

\subsubsection{Model architecture}

State variables: Here we model the foraging decisions of an organism as a function of two principle state variables: 1) the organism's net energy reserves at time $t, X(t)=x$, and 2) the organism's enamel volume at time $t, V(t)=v$, where time $t$ is measured in days. The net energy reserves of an organism determine the amount of energy available to it for performing life-sustaining tasks (e.g. foraging). We model a single unit of energy as 10 Megajoules [MJ], equivalent to $2388 \mathrm{kcal}$ and roughly equal to the energy in $1 \mathrm{~kg}$ of animal tissue [152]. Accordingly, the maximum potential energy reserves for an organism, $x_{\max }$, is its maximum body size, such that $x_{\max }=70$ for a $70 \mathrm{~kg}$ organism. Because an organism cannot use all of the energy contained in its mass, we set a critical threshold for an organism's energy reserves $\left(x_{c}\right)$ below which it cannot survive. We define this lower energy bound as $x_{c}=(3 / 4) x_{\max }$. By contrast, an organism's enamel volume accounts for the volume of molar enamel, where a single unit of $v$ corresponds to $100 \mathrm{~mm}^{3}$ (equivalent to a sphere with a $2.9 \mathrm{~mm}$ radius). Among anthropoids, specific properties of molar anatomy correlate strongly with body size, and these properties can be used to approximate maximal molar enamel volume, $v_{\max }$ as a function of $x_{\max }$.

The length of the enamel-dentine junction $(L)$ and the average enamel thickness $(\epsilon)$ of molars $\mathrm{M}_{1}, \mathrm{M}_{2}$, and $\mathrm{M}_{3}$ (the first, second, and third mandibular molars), have strong allometric relationships among anthropoids of the form $y=a x_{\max }^{b}$ [165], 
where $a$ and $b$ are allometric constants. The enamel-dentine junction length correlates with the square root of dentine area $(\sqrt{D})$, which itself correlates to body size, and can be approximated by

$$
L_{m}=a_{1, m}{\sqrt{D_{m}}}^{b_{1, m}}, \text { and } \sqrt{D_{m}}=a_{2, m} x_{\max }^{b_{2, m}}
$$

for $m=1,2,3$, corresponding to measurements for the molars $\mathrm{M}_{1}, \mathrm{M}_{2}$, and $\mathrm{M}_{3}$, respectively. The allometric measurements for the molars $\mathrm{M}_{1}, \mathrm{M}_{2}$, and $\mathrm{M}_{3}$ are $a_{1, m}=$ $(3.58,3.38,3.21), a_{2, m}=(1.93,1.94,1.97), b_{1, m}=(0.93,0.99,1.00)$, and $b_{2, m}=$ $(0.33,0.35,0.35)[165]$. Similarly, average enamel thickness can be estimated as a function of body size, such that

$$
\epsilon=a_{3, m} x_{\max }^{b_{3, m}}
$$

where $a_{3, m}=(0.19,0.21,0.16)$ and $b_{3, m}=(0.43,0.43,0.55)$ [165]. Applying the simplifying assumption that the shape of the organism's molars are symmetrical (with respect to an axis perpendicular to the occlusal surface) and mandibular and maxillary molar enamel volume is equivalent, total molar enamel volume is

$$
v_{\max }=4 \sum_{m=1}^{3} \frac{\pi}{4} \epsilon_{m}\left(x_{\max }\right) L_{m}^{2}\left(x_{\max }\right)=\sum_{m=1}^{3} \pi \epsilon_{m}\left(x_{\max }\right) L_{m}^{2}\left(x_{\max }\right) .
$$

Here, $\epsilon$ (the average enamel thickness) and $L$ (the length of the enamel-dentine junction) are written as functions of body size $x_{\max }$. The middle equation is the volume for a cylinder with a radius of $L / 2$ and length $\epsilon$ multiplied by 4 , such that the right, 
left, mandibular, and maxillary molars contribute to total molar volume (see Fig. S4.1). For example, an $50 \mathrm{~kg}$ anthropoid is predicted to have a molar enamel volume of 6479 $\mathrm{mm}^{3}$, equivalent to a sphere with a $11.5 \mathrm{~mm}$ radius (similar to the $10 \mathrm{~mm}$ estimate proposed by [102]). Given an enamel density of ca. $2.9 \mathrm{gcm}^{-3}$ [110], this sphere would weigh ca. 18.8 grams. By contrast, megadont hominins, such as Paranthropus robustus had greater-than-predicted enamel thickness. To contrast the foraging decisions of the 'average anthropoid primate' with those of megadont hominins, we substituted enamel thickness values measured for $P$. robustus, where $\epsilon=2.5,3.1$, and $3.1 \mathrm{~mm}$ for molars $\mathrm{M}_{1}, \mathrm{M}_{2}$, and $\mathrm{M}_{3}$ (we again assume mandibular and maxillary molars are equivalent) [162]. Compared to an 'average anthropoid', the enamel volume of a megadont hominin is estimated as ca. $16013 \mathrm{~mm}^{3}$, with a mass of ca. 46.4 grams. An organism requires a critical amount of enamel to effectively masticate food. We define the critical threshold of enamel volume $\left(v_{c}\right)$ required for survival as the volume of enamel for at least one molar on each tooth-row, such that $v_{c}=\pi \epsilon\left(x_{\max }\right) L^{2}\left(x_{\max }\right)$, where values of $\epsilon\left(x_{\max }\right)$ and $L\left(x_{\max }\right)$ are conservatively estimated for the $\mathrm{M}_{1}$ (smallest) molar.

Mechanical properties: The potential energy gained from food, as well as its impact on an organism's enamel, change as a function of the food's mechanical properties. Here we consider an approximating measurement for the mechanical properties of food that takes into account both the elasticity (Young's modulus, $E_{i},[\mathrm{MPa}]$ ) and fracture toughness $\left(R_{i},\left[\mathrm{Jm}^{-2}\right]\right)$ of food $i$, and is generally assumed to approximate 'hardness', measured as $\sqrt{E_{i} R_{i}}[102]$. Moreover, the toughness of a food scales roughly with the amount of fiber [103]; it is reasonable to consider foods with high toughness 
as less digestible. We let $\eta_{i}$ denote the digestibility of food $i$ ranging between $\eta_{i}=0$ (indigestible) and $\eta_{i}=1$ (completely digestible).

Gains: An organism's energetic gains and foraging costs are denoted as $\gamma_{i}$, and $c_{i}$, respectively, for a given food $i$. Here, we identify four general food groups: 1) a nutritious, non-fracture resistant, patchily distributed food (e.g. fruit), 2) a nonnutritious, fracture resistant, widely distributed food (e.g. grass leaves), 3) a nutritious, fracture resistant, widely distributed food (e.g. USOs), and 4) a highly-nutritious, potentially fracture resistant, patchily distributed food (e.g. arthropods or more generally small quantities of animal protein). We set the energetic gain associated with each food group to be $717,150,785$, and $1518 \mathrm{kcal} / \mathrm{kg}$ for fruit, grass leaves, USOs, and arthropods/animal protein [152]. Because larger animals gain relatively more calories per foraging bout, energetic gain $\gamma_{i}=($ food gain $/ 2388) \cdot\left(x_{\max } / 10\right)$, where the constant $(1 / 2388)$ ensures $\gamma_{i}$ is in units of $x$, and the constant $(1 / 10)$ ensures that gain is normalized to the smallest considered body size $\left(x_{\max }=10\right)$.

Costs: We model the daily cost of foraging for food type $i, c_{i}$, as a function of an organism's body size, and the aggregation of food on the landscape. Foraging increases nonlinearly with body size, such that larger organisms expend a smaller fraction of energy than do smaller organisms [96]. We used the allometric relationship proposed by [96], such that $c_{i}=C_{1} \cdot \mathrm{RMR} \cdot\left(1 / \xi_{i}\right) / 2388$, and $\mathrm{RMR}=69.1 x_{\max }^{0.761}$, where $C_{1}$ is the activity constant $\left(C_{1}=3.80\right.$ for moderate activity), the constant $(1 / 2388)$ operates as above, and $\xi_{i}$ is the mean encounter rate for food $i$, such that $\left(1 / \xi_{i}\right)$ is proportional to foraging time (see below for a detailed description of encounter rates). Therefore, 
foods that are encountered more frequently (high $\xi_{i}$ ) have lower per encounter foraging costs. In addition, we assessed a second, costlier, version of the model, where $c_{i}=$ $\left(C_{1} \cdot \mathrm{RMR} \cdot\left(1 / \xi_{i}\right)+C_{2} \cdot \mathrm{RMR}\right) / 2388$, where $C_{1}$ is defined as before, and $C_{2}=1.2$, accounting for additional daily costs independent of food choice.

Mechanical Advantage: An organism's potential mechanical advantages are incorporated by modifying the mechanical properties of particular food resources. If an organism has no mechanical advantage, foods have unmodified mechanical properties. Accordingly, values for toughness $\left[\mathrm{Jm}^{-2}\right]$ are $R=(561,300,265,1345)$, and Young's modulus $[\mathrm{MPa}]$ are $E=(1,11,5,200)$ for fruits, grass leaves, USOs, and arthropods, respectively [104, 199, 51]. We note that the high value of $R$ for arthropods is due to tough exoskeletons. Many animals, and primates in particular, can modify the mechanical properties of foods before they are consumed. We consider two potential mechanical advantages: 1) peeling: such that the arthropod exoskeleton is removed $\left(R_{\text {arthropod }}=306\right.$ and $\left.E_{\text {arthropod }}=0.22\right)$ (alternatively, this mechanical advantage can account for swallowing arthropods without chewing), and 2) peeling + pounding or cooking: such that both arthropod exoskeletons are peeled and USOs are either pounded or cooked $\left(R_{\text {arthropod }}=306\right.$ and $E_{\text {arthropod }}=0.22$, and $R_{\mathrm{USO}}=138$ and $\left.E_{\mathrm{USO}}=5[51]\right)$.

Habitat Quality: The quality of a habitat at a given time, $Q(t)$, is variable, and this affects both the nutritional gains and foraging costs of foods. We model quality for rich $(Q(t)=\mathrm{r})$ or poor $(Q(t)=\mathrm{p})$ habitats, such that habitat quality $Q(t)$ changes in accordance to a transition probability matrix $(\boldsymbol{\rho})$ where 


$$
\boldsymbol{\rho}=\left(\begin{array}{cc}
\rho_{\mathrm{rr}} & \rho_{\mathrm{rp}} \\
\rho_{\mathrm{pr}} & \rho_{\mathrm{pp}}
\end{array}\right),
$$

and, for example, $\rho_{\mathrm{rp}}$ is the probability of transitioning from a rich quality to a poor quality habitat. Caloric gain in rich quality habitats has the caloric gain described above, whereas the caloric gain of food in poor quality habitats is decreased by $10 \%$. Moreover, the mean encounter rate $\left(\xi_{i}\right)$ as well as the dispersion of food $\left(\nu_{i}\right)$ change as a function of habitat quality, such that food resources are more easily found in rich quality habitats. USOs are stored underground and have evolved to retain high nutrient loads during periods of environmental stress [46]. We incorporate this quality by holding the caloric gain, encounter rate, and dispersion of USOs constant, irrespective of habitat quality. With this basic framework, we can asses the influence of 'wet', and 'dry' environments on foraging decisions. Wet environments have high values of $\rho_{\mathrm{rr}}, \rho_{\mathrm{pr}}$, and low values of

$\rho_{\mathrm{rp}}, \rho_{\mathrm{pp}}$ (such that food quality is generally high quality), while dry environments have low values of $\rho_{\mathrm{rr}}, \rho_{\mathrm{pr}}$, and high values of $\rho_{\mathrm{rp}}, \rho_{\mathrm{pp}}$ (such that food quality is generally low quality). We acknowledge that natural systems are more idiosyncratic, however this simplification allows us to assess the effects of changing food quality over time with minimal added complexity.

\subsubsection{State dynamics}

The dynamics of the SDP are determined by how the state variables $X$ and $V$ change as a function of the gains and costs associated with foraging for different foods 
$i$ at time $t$. Because an organism's daily consumption is limited by stomach size, daily caloric gain is bounded by daily stomach capacity, $x_{s}=(1 / 5) \cdot x_{\max }$ (proportional to average anthropoid \% gut volume; [121]). Accordingly, an organism's energy reserves change according to the difference equation

$$
X_{\mathrm{r}, \mathrm{p}}(t+1)=X_{\mathrm{r}, \mathrm{p}}(t)+\min \left(k \eta_{i} \gamma_{i}, x_{s}\right)_{\mathrm{r}, \mathrm{p}}-c_{i}
$$

with probability $f_{K}(k)_{i}$, the probability an organism finds $k$ items of food $i$. The notation $f_{K}$ denotes the frequency distribution for the random variable $K$, whereas $k$ takes a specific value of $K$ (this notation is retained for all stochastic variables).

The volume of enamel decreases as an animal consumes resources. The proximal mechanisms that cause enamel wear are not well understood [105], but likely include silica or phytoliths found within plant foods, exogenous grit or dirt on the outside of plant foods (or the inside of some insects), and/or tooth-on-tooth wear [104, 102, 150]. We make the assumption that hard/tough foods promote increased use of the dentition, and this increased use promotes wear regardless of the specific cause. Accordingly, we set enamel wear, $\Delta v(\Omega)$, to be a function of: 1) the mechanical properties of food $i$ and 2) a stochastic decrease in enamel volume (determined by the random variable $\Omega$ ). Because enamel is a nonrenewable resource, this wear cannot be undone. Teaford (1986) showed that vervet monkeys (Chlorocebus) fed on a diet of raw Purina Monkey Chow required 8x greater consumption time compared to monkeys fed on pre-mashed Monkey Chow. With respect to enamel, this is equivalent to chewing $8 \mathrm{x}$ as much food. Teaford also showed that the enamel thickness decreased by ca. $0.58 \mu \mathrm{m} \mathrm{day}^{-1}$ when 
fed on the raw diet, versus ca. $0.24 \mu \mathrm{m} \mathrm{day}^{-1}$ when fed on the pre-mashed diet. A linear relationship can be derived between the loss of enamel thickness, and the amount of food consumed, $k$ (with a slope $b=0.0425$ ). The y-intercept of the relationship between enamel thickness decline and the amount of food consumed $(\bar{\omega}=0.24 \mu \mathrm{m})$ represents the expected basal enamel wear that occurs irrespective of consumption, and is used to parameterize the stochastic variable $\Omega$ (see below). Accordingly, given that $A$ is the molar enamel surface area and $E_{M C}$ and $R_{M C}$ are scaling constants denoting the average Young's modulus (50.44 MPa) and fracture toughness $\left(1030.55 \mathrm{Jm}^{-2}\right)$ of monkey chow, respectively [199], enamel volume changes according to the difference equation

$$
V(t+1)=V(t)-\underbrace{\frac{A}{250}\left(\frac{b k \sqrt{E_{i} R_{i}}}{\sqrt{E_{M C} R_{M C}}}+\Omega\right)}_{\Delta v},
$$

The constant $1 / 250$ scales tooth wear to ensure the organism attains its expected longevity [98], and accounts for 1) overestimation of molar enamel area (our allometric estimation includes the lateral aspects of molar surfaces), and 2) the fact that wear is a complex action affecting a small fraction of the occlusal surface at a time [104].

The basal rate of enamel wear, $\Omega$, is a stochastic variable with an expected value $\mathrm{E}(\Omega)=\bar{\omega}=0.24 \mu \mathrm{m}$. As such, chewing, as well as daily wear unassociated with chewing [104], have variable effects on enamel wear. Specifically, enamel wear should typically be small, but occasionally be large, realized when the organism chips or looses a tooth or part of a tooth. The probability that the random variable $\Omega$ falls within the 
small interval $\omega$ and $\omega+\mathrm{d} \omega$ is modeled as a lognormal distribution [20],

$$
\begin{aligned}
f_{\Omega}(\omega) & =\log \operatorname{Normal}(\omega \mid \bar{\omega}, \sigma) \\
& =\frac{1}{\omega \sigma \sqrt{2 \pi}} \exp \left(-\frac{(\ln \omega-\bar{\omega})^{2}}{2 \sigma^{2}}\right),
\end{aligned}
$$

where $\mathrm{E}(\Omega)=\bar{\omega}$ and $\operatorname{Var}(\Omega)=\sigma^{2}$.

\subsubsection{Aggregation of food resources}

The probability of finding $k$ items of food $i$ varies as a function of its distribution on the landscape. Some foods are more evenly distributed, such that the encounter rate does not vary, while other foods are patchily distributed and have unpredictable encounter rates. To incorporate landscape variability without establishing an explicit spatial framework, the probability of encountering $K=k$ items of food $i$ is first modeled as a Poisson distribution, which is discrete and defined for positive integers, such that

$$
\begin{aligned}
f_{K}(k)_{i}=\operatorname{Pr}\left(K_{i}=k_{i}\right) & =\text { Poisson }\left(k_{i} \mid \lambda_{i}\right) \\
& =\frac{\lambda_{i}^{k_{i}} \exp \left(-\lambda_{i}\right)}{k_{i} !},
\end{aligned}
$$

where $\lambda_{i}$ describes the encounter rate, and $\mathrm{E}\left(K_{i}\right)=\operatorname{Var}\left(K_{i}\right)=\lambda_{i}$. If the rate that a food item is found varies, the encounter rate is modeled as a gamma distribution, which is continuous and defined for positive real numbers, such that 


$$
\begin{aligned}
f_{\Lambda}(\lambda)_{i} & =\operatorname{Gamma}\left(\lambda_{i} \mid \alpha_{i}, \nu_{i}\right) \\
& =\frac{\alpha_{i}^{\nu_{i}}}{\Gamma\left(\nu_{i}\right)} \exp \left(-\alpha_{i} \lambda_{i}\right) \lambda_{i}^{\left(\nu_{i}-1\right)}
\end{aligned}
$$

where $\mathrm{E}\left(\Lambda_{i}\right)=\nu_{i} / \alpha_{i}, \operatorname{Var}\left(\Lambda_{i}\right)=\nu_{i} / \alpha_{i}^{2}$, and the gamma function $\Gamma\left(\nu_{i}\right)=\left(\nu_{i}-1\right)$ !. From Eq. (4.9), $\alpha_{i}$ must be a time-valued parameter, while $\nu_{i}$ is dimensionless. To determine the meaning of $\nu_{i}$, we note that the coefficient of variation $\left(\mathrm{CV}\left(\Lambda_{\mathrm{i}}\right)=\sqrt{\operatorname{Var}\left(\Lambda_{\mathrm{i}}\right)} / \mathrm{E}\left(\Lambda_{\mathrm{i}}\right)=\right.$ $\left.1 / \sqrt{\nu_{i}}\right)$ collapses to zero if $\mathrm{E}\left(\Lambda_{i}\right)$ is held constant and $\nu_{i} \rightarrow \infty$. This means that as $\nu_{i}$ increases, the probability density of $\lambda_{i}$ becomes infinitely dense around the mean. The parameter $\nu_{i}$ thus describes the 'dispersion' of $\lambda_{i}[70]$.

To calculate the probability that $K_{i}=k_{i}$, we multiply Eq. (4.8) by Eq. (4.9) and integrate across all potential values of $\Lambda_{i}$ to obtain a negative binomial distribution,

$$
\begin{aligned}
f_{K}(k)_{i}=\operatorname{Pr}\left(K_{i}=k_{i}\right) & =\int_{0}^{\infty} \frac{\lambda_{i}^{k_{i}} \exp \left(-\lambda_{i}\right)}{k_{i} !} \frac{\alpha_{i}^{\nu_{i}}}{\Gamma\left(\nu_{i}\right)} \exp \left(-\alpha_{i} \lambda_{i}\right) \lambda_{i}^{\left(\nu_{i}-1\right)} \mathrm{d} \lambda_{i} \\
& =\frac{\Gamma\left(\nu_{i}+k_{i}\right)}{\Gamma\left(\nu_{i}\right) k_{i} !}\left(\frac{1}{\alpha_{i}+1}\right)^{k_{i}}\left(\frac{\alpha_{i}}{\alpha_{i}+1}\right)^{\nu_{i}} \\
& =\operatorname{NegBin}\left(k_{i} \mid \nu_{i}, \alpha_{i}\right)
\end{aligned}
$$

where $\mathrm{E}\left(K_{i}\right)=\xi_{i}=\nu_{i} / \alpha_{i}$ and $\operatorname{Var}\left(K_{i}\right)=\nu_{i} / \alpha_{i}+\nu_{i} / \alpha_{i}^{2}$. The parameters $\nu_{i}$ and $\alpha_{i}$ are now interpreted to describe the dispersion of food $i$ on the landscape, and the relative time separating encounters, respectively. As the time between encountering food items $\left(\alpha_{i}\right)$ decreases, the rate at which they are found increases. Moreover, if the dispersion parameter $\nu \gg 0$ for food $i$, the encounter rate $\lambda$ collapses to its mean value such 
that $K$ is distributed as a Poisson distribution with $\lambda=\xi=\nu / \alpha$, such that food is distributed evenly. By contrast, as $\nu \rightarrow 0$ for food $i, \lambda$ becomes more variable, meaning the probability of finding $k$ items of food $i$ is less predictable, equivalent to foraging on a patchy landscape. We ranked values of $\xi_{i}$ and $\nu_{i}$ according to qualitative relationships between different resources commonly foraged by non-human primates [61], such that in high quality habitats, $\xi=(3,4,3,1)$ and $\nu=(3,5,3,2)$ and in low quality habitats, $\xi=(2,4,3,1)$ and $\nu=(2,4,3,1)$ for fruits, grass leaves, plant USOs, and arthropods, respectively. We also explored a second scenario, where the relative abundance of grass leaves is exaggerated, such that $\xi_{\text {leaves }}=5$.

\section{Maximizing fitness from food choice}

We have described the conditions by which the state variables $x$ and $v$ change over time. By integrating the above dynamics into a cumulative fitness function, we determine an organism's optimal foraging decisions as a function of its current state at time $t$. Fitness is a function $x, v$ and $t$, and is calculated for both rich and poor quality habitats $\left(F_{\mathrm{r}}(x, v, t)\right.$ and $\left.F_{\mathrm{p}}(x, v, t)\right)$ separately, where the probability of transitioning from one habitat quality to another $(\boldsymbol{\rho})$ is integrated into each fitness function. Fitness maximization is solved in accordance to a backwards iteration algorithm and iteratively solved from the terminal timestep $t=T$ to $t=1$ [109, 41]. Fitness-maximizing decisions are often strongly influenced by the terminal fitness function when $t$ is close to $T$. Because the organisms in our model are long-lived, we assume daily foraging decisions are independent of terminal time fitness (i.e. what an organism chooses to eat today 
has nothing to do with its fitness in many years).

To derive an equation $F(x, v)$ that is independent of time $t$, we first solve $F(x, v, t)$ for $t \ll T$, which approximates the stationary solution; the time variable serves only to provide a means by which the stationary equation can be solved $[117,109]$. Stationary solutions will result in decision matrices with foods chosen only as a function of $x$ and $v$. To determine the stationary solutions $F^{*}(x, v)$ we must first define the terminal fitness function. Terminal fitness is defined as an increasing function of both $x$ and $v$, where organisms with higher energy reserves and enamel volume have greater potential future reproductive fitness, such that

$$
F_{\mathrm{r}}(x, v, T)=F_{\mathrm{p}}(x, v, T)=\Phi(x, v)
$$

and

$$
\begin{array}{ll}
\Phi(x, v)=\frac{1}{2}\left(2-\frac{x_{c}}{x}-\frac{v_{c}}{v}\right), & \text { where }\left\{\begin{array}{c}
x>x_{c} \\
v>v_{c}
\end{array}\right. \\
\Phi(x, v)=0 & \text { otherwise. }
\end{array}
$$

The maximum fitness at $t=T$ is realized by an organism with $X(T)=x_{\max }$ and $V(T)=v_{\max }$, while the rate of fitness decline increases as $x$ and $v$ approach $x_{c}$ and $v_{c}$. The fitness functions $F_{\mathrm{r}}(x, v, t)$ and $F_{\mathrm{p}}(x, v, t)$ describe the maximum expected lifetime fitness given the state variables $x, v$, and the time $t$ in rich quality and poor quality habitats, respectively, such that 


$$
\begin{aligned}
& F_{\mathrm{r}}(x, v, t)=\max _{i} \mathrm{E}\{\Phi(X(T), V(T)) \mid X(t)=x, V(t)=v, Q(t)=\mathrm{r}\} \\
& F_{\mathrm{p}}(x, v, t)=\max _{i} \mathrm{E}\{\Phi(X(T), V(T)) \mid X(t)=x, V(t)=v, Q(t)=\mathrm{p}\}
\end{aligned}
$$

For time periods previous to the terminal time $t=T$, rich quality and poor quality fitness are determined by the canonical equations for optimal food selection,

$$
\begin{aligned}
F_{\mathrm{r}}(x, v, t)= & \max _{i}\left(\sum _ { k = 0 } ^ { k _ { \operatorname { m a x } } } f ( k _ { \mathrm { r } } ) _ { i } \left(\rho_{\mathrm{rr}} \mathrm{E}_{\Omega}\left\{F_{\mathrm{r}}\left(x_{\mathrm{r}}+\min \left(k \eta_{i} \gamma_{i}, x_{s}\right)_{\mathrm{r}}-\left(c_{i}\right)_{\mathrm{r}}, v-\Delta v(\Omega), t+1\right)\right\}\right.\right. \\
& \left.\left.+\rho_{\mathrm{rp}} \mathrm{E}_{\Omega}\left\{F_{\mathrm{p}}\left(x_{\mathrm{r}}+\min \left(k \eta_{i} \gamma_{i}, x_{s}\right)_{\mathrm{r}}-\left(c_{i}\right)_{\mathrm{r}}, v-\Delta v(\Omega), t+1\right)\right\}\right)\right)(1-d),
\end{aligned}
$$

and

$$
\begin{aligned}
F_{\mathrm{p}}(x, v, t)= & \max _{i}\left(\sum _ { k = 0 } ^ { k _ { \operatorname { m a x } } } f ( k _ { \mathrm { p } } ) _ { i } \left(\rho_{\mathrm{pr}} \mathrm{E}_{\Omega}\left\{F_{\mathrm{r}}\left(x_{\mathrm{p}}+\min \left(k \eta_{i} \gamma_{i}, x_{s}\right)_{\mathrm{p}}-\left(c_{i}\right)_{\mathrm{p}}, v-\Delta v(\Omega), t+1\right)\right\}\right.\right. \\
& \left.\left.+\rho_{\mathrm{pp}} \mathrm{E}_{\Omega}\left\{F_{\mathrm{p}}\left(x_{\mathrm{p}}+\min \left(k \eta_{i} \gamma_{i}, x_{s}\right)_{\mathrm{p}}-\left(c_{i}\right)_{\mathrm{p}}, v-\Delta v(\Omega), t+1\right)\right\}\right)\right)(1-d),
\end{aligned}
$$

where the expectation $\mathrm{E}$ is with respect to the random variable $\Omega$ (Eq. 4.6), and $d$ is the probability of mortality, where $d=4.54 \times 10^{-5}$ [34]. This equation identifies the food $i$ that maximizes fitness for a given energy reserves $X(t)=x$ and enamel volume $V(t)=v$. Moreover, optimal food selection takes into account the probabilistic quantities of finding $K$ items of food $i$, (from the frequency distribution $\left.f_{K}(k)_{i}\right)$ and losing a basal amount 
of enamel volume $\Omega$, (from the frequency distribution $f_{\Omega}(\omega)$ ). Decision matrices are derived by solving for the stationary solutions to the above equations, $F_{\mathrm{r}}^{*}(x, v)$ and $F_{\mathrm{p}}^{*}(x, v)$, such that a single food maximizes fitness for a given internal state $(x, v)$.

\subsection{Results and Discussion}

Foraging decisions are often complex and multi-faceted, and chosen foods must balance many potential costs and benefits. Although the number of internal and external states used to inform foraging decisions is vast, some vital states are more important than others. Prominent morphological differences between closely related species provide important clues regarding key selective pressures that influence behavior. Among anthropoid primates, and hominins in particular, the robustness of molar enamel is one such morphological trait $[82,201]$.

Although model-based approaches have been used to investigate the foraging strategies of human primates [16], non-human primates [32, 161], and even the interactions between the two [97], few have been applied to extinct primates (but see $[53,76,63])$, and to our knowledge none have taken into account nonrenewable resources such as dental enamel. This is surprising given the prominence prescribed to dental morphology, and its known impact on diet. Stochastic dynamic programs demand the explicit expression of processes by which optimal decisions are made, as well as the influence of both external and internal stochasticity [109, 41]. Our goal is to not create an agent-based demographic model of anthropoid foraging behavior, but to assess 
directly the dependence of diet choice on enamel state, and to quantify the extent that attributes such as mechanical advantage or megadont dentition alter foraging decisions.

Here we determine under what conditions fracture resistant foods such as USOs are chosen as fallback resources, and whether and to what extent advantages capable of altering the mechanical properties of foods convey fitness benefits. We then contrast the potential benefits of mechanically altering foods prior to consumption with the predicted benefits of having a megadont dentition, where enamel is thicker than expected from allometric relationships between enamel and body size. Our model results suggest that the contradictory patterns observed in the stable isotope ratios and microwear patterns of early hominins are directly related to changing foraging behavior as enamel is worn, and we end by showing how our model can be used as both a predictive tool for understanding hominin diet, as well as an investigative tool for determining the conditions under which empirical observations are predicted.

\subsubsection{Fitness-maximizing foraging decisions}

Multiple lines of evidence suggest that plant USOs played an important role in hominin diet. Plants with geophytic structures are both diverse and abundant in arid habitats $[142,195,149]$, and modern hunter-gatherers utilize these resources extensively, particularly in marginal environments [111]. Associations between mole rats - known USO specialists - and hominins suggest that they lived in USO-abundant areas [93], while stable isotope analysis of both modern and fossil mole rats confirm that USO specialists have isotopic values similar to those of Australopithecus africanus and 
Paranthropus robustus [204]. It has generally been assumed that USOs serve as fallback rather than preferred foods, due to their lower nutritional content and relative availability. The results of our model are in agreement with this assumption, and show that this fallback dependency is - in part - a function of an organism's enamel volume.

The stationary solutions of the stochastic dynamic program (Eqs. 4.13 and 4.14) show that energy reserves, $x$, and enamel volume, $v$, have large consequences on diet choice (shown for a $50 \mathrm{~kg}$ anthropoid primate; Fig. 4.1). Moreover, resource quality (rich vs. poor habitats) has a similarly large effect on foraging strategies. For animals without mechanical advantage, fruit maximizes fitness for all potential states $(x, v)$ in rich quality habitats (Fig. 4.1A). In poor quality habitats, fruits are chosen only if energy reserves are close to $x_{\max }$ (Fig. 4.1B); as energy reserves decline the optimal resource switches from fruit to plant USOs. However, as enamel volume declines, the mechanical hardness of USOs negatively impacts fitness. Plant USOs are less nutritious than fruit, but are also less patchy, and the probability of finding $k$ USOs is generally higher than it is for fruits. Our model predicts that USOs serve as a fallback resource when the organism is physiologically stressed, particularly in poor quality habitats. However, the fitness benefits of USOs are constrained by enamel volume, and USOs are chosen less as enamel is worn.

Because foraging costs scale nonlinearly with body size, optimal foraging decisions may be expected to vary with energy reserves and enamel volume for differently sized animals. Our model predicts animals with larger body sizes to consume a larger proportion of riskier foods that have greater nutritional yield (in this case, fruit) during 
periods of environmental stress (Fig. 4.2A). As body size decreases, the proportional contribution of less nutritious but less risky foods (USOs) increases. Small animals $\left(x_{\max }=10 \mathrm{~kg}\right)$ maximize fitness by consuming fruit irrespective of energy reserves or enamel volume in high quality habitats, while in low quality habitats USO consumption always maximizes fitness. Thus, our model predicts a tradeoff with respect to the fitness benefits of fallback foods. In low quality habitats (when fallback foods are expected to be utilized) the percentage of internal states $(x, v)$ predicting USOs as optimal foods declines with increasing body size (Fig. 4.2A) because larger animals risk less when foraging for costlier foods. Moreover, foraging for less abundant, nutritious, and non-fracture resistant foods such as fruit entirely depends on enamel volume. When enamel volume is high (such that its preservation is not a concern), USOs are more likely to maximize fitness for a given energetic state (Fig. 4.1B). Our results suggest that fallback foods are of maximal utility for smaller animals, and are only important for large animals when energy reserves are low. For very large animals, factors such as increased digestive efficiency are expected to counterbalance the benefits of risky foraging, and the advantages such adaptations impart would be worth exploring using a SDP approach. Although hominins are expected to have less complex hind-gut fermenting gut physiologies [121], they were likely capable of mechanically altering their food prior to consumption. We find that such mechanical advantages strongly impact foraging decisions.

We examine the effects of two potential mechanical advantages that may or may not require the use of tools: 1) the ability to extract arthropods from their hard 
chitinous exoskeletons, and 2) the ability to both extract arthropods and either pound or cook plant USOs. Although the above scenarios were expected to produce distinct decision matrices, we found that the fitness maximizing solutions were strongly similar (Fig. 4.2B,C), but differed from the 'no mechanical advantage' scenario (Fig. 4.2A). For either mechanical advantage, as body size increases the USOs are reduced as before, however arthropods tend to become favored over fruit. Thus, in both rich and poor quality habitats, large-bodied anthropoid primates are expected to increase the percentage of highly risky foods (for a given internal state) if the mechanical properties of food can be altered such that enamel is preserved (Fig. S4.2). Although the proportional contribution of foraging choices represented in decision matrices is an efficient summary of potential dietary behavior, it should not be viewed as the proportional contribution to diet, which can be quantified using a forward iteration algorithm (see below). Our results predict that physically altering the mechanical properties of foods, which conserves enamel volume, does increase potential dietary diversity. It stands to reason that the evolution of megadont dentition, which increases the quantity of enamel volume for a given body size, may serve a similar function.

Megadont dentition is a prominent morphological feature among robust hominins, though its fitness benefits are a source of contention. Hominins with megadont dentition ranged from 2.5 to 1.1 Ma, had smaller incisors and canines, yet hyper-thickly enameled molars [105]. Megadont hominins had greater quantities of molar enamel than expected [165], and may exhibit greater tolerance for fracture resistant foods $[82,136,108]$. This may have evolved in response to hard-object feeding, possibly on 
grass seeds [78] or plant USOs [176, 93]. We assessed the relative impact of megadont dentition on predicted foraging choices for a $50 \mathrm{~kg}$ anthropoid primate with the enamel thickness of $P$. robustus (see Methods).

Our analysis shows that in poor quality habitats, megadont primates maximize fitness by incorporating a greater proportion of fracture resistant foods than do non-megadonts. With no mechanical advantage, megadont primate decision matrices show a reduction in the proportional contribution of fruit, and an increase in fracture resistant USOs relative to non-megadonts (Fig. 4.3). When the arthropod mechanical advantage is introduced, the percentage of USOs increase for megadont primates, while the proportion of arthropods in the decision matrices is increased overall, but reduced relative to non-megadonts. Similarly, when the arthropod + USO mechanical advantage is introduced, the proportional contribution of arthropods is deceased, while that of USOs is increased relative to non-megadonts. Because our model predicts that megadont primate decision matrices have a larger percentage of fracture resistant foods, it suggests that this morphological feature would convey fitness benefits in stressed, or poor habitat quality, conditions.

\section{The Fitness Benefits of Mechanical Advantage}

Mechanical advantages are expected to provide fitness benefits, particularly in environments where harder or tougher foods are more abundant. The stationary solutions to the dynamic programming equations, $F_{\mathrm{r}}^{*}(x, v)$ and $F_{\mathrm{p}}^{*}(x, v)$ (Eqs. 4.13 and 4.14), identify foods that maximize fitness for a given state $(x, v)$ during both rich 
quality (r) and poor quality (p) environmental conditions. We used a forward iteration algorithm $[109,41]$ to assess the impact that these foraging decisions have on individuals by iteratively solving for the state dynamics of simulated foragers over time, as specified by Eqns. 4.5 and 4.6 .

To test whether alternative mechanical advantages contributed fitness benefits to non-megadont or megadont primates, we calculated expected fitness across $N=100$, $50 \mathrm{~kg}$ individuals, with maximal foraging costs and for days $\tau=1$ to $\tau_{\max }=10950$ (lifespan of 30 years) given rich $(Q=\mathrm{r})$ or poor $(Q=\mathrm{p})$ habitat conditions, $\hat{F}(\tau \mid Q(\tau))$. As energy reserves or enamel volume decrease over the lifetime of an individual, $\hat{F}(\tau \mid Q(\tau))$ is expected to similarly decrease. Populations with mechanical advantages or enamel properties that result in relatively higher $\hat{F}(\tau \mid Q(\tau))$ have greater fitness advantages compared to those populations with lower $\hat{F}(\tau \mid Q(\tau))$. Expected fitness at time $\tau$, given rich or poor quality habitat conditions, was quantified as

$$
\hat{F}(\tau \mid Q(\tau))=\frac{1}{N} \sum_{n=1}^{N} F^{*}\left(X_{n}(\tau), V_{n}(\tau), \tau \mid Q(\tau)\right) .
$$

We calculated $\hat{F}(\tau \mid Q(\tau))$ for non-megadonts and megadonts and for the three mechanical advantage scenarios in both wet periods (where rich quality habitats are more likely) and dry periods (where poor quality habitats are more likely).

For all populations, fitness decreases more sharply early in life, but saturates as the population nears its expected lifespan (Fig. 4.4). Our results reveal an important difference between the three mechanical advantage scenarios (none, arthropods, arthropods + USOs) that is less evident when comparing decision matrices, particularly for 
non-megadont primates (Fig. 4.4A,B; solid lines). Although the decision matrices for the arthropod and arthropod + USO scenarios are similar for non-megadont primates, mechanical advantages have large impacts on expected fitness, conditional on environmental conditions. During both wet and dry periods, having either mechanical advantage provides large fitness benefits, but the difference in fitness between mechanical advantage scenarios is small, particularly during wet periods. During dry periods, the fitness difference between the arthropod and arthropod + USO scenario is exaggerated, and having greater mechanical advantage generally results in higher expected fitness.

The fitness benefits of megadont dentition are more obvious. Megadont populations have greater expected fitness than those of non-megadonts - irrespective of mechanical advantage - and these differences are more exaggerated later in life (Fig. 4.4A,B; stippled lines). Moreover, the benefits conveyed by mechanical advantage have less impact on fitness for megadonts than non-megadonts. During dry periods, the expected fitness of populations without mechanical advantage is greater than those with mechanical advantages early in life, but relatively lower later in life. Importantly, our model predicts that the fitness benefits provided by mechanical advantage are generally less for megadont populations than those for non-megadont populations.

Fitness changes over an individual's lifetime, and is expected to diverge as enamel wears at different rates. Our process-based model is simplistic in that lifehistory stages are not included, such that energy reserves and enamel wear are the only time-dependent variables. These simplifications allow us to make a number of predictions regarding foraging behavior. First, behaviors that alter the mechanical properties 
of fracture resistant foods should result in greater fitness. Second, these benefits are primarily realized in environments where fracture resistant foods are both nutritious and prevalent. Third, because megadont dentition results in relatively slower wear rates, megadonts have greater expected fitness, but these benefits are primarily realized later in life. Moreover, we observe that the cumulative fitness differences between mechanical advantage scenarios for megadont primates are less emphasized than for non-megadonts. This suggests that megadont dentition is a suitable replacement for, rather than an addition to, behavioral actions aimed at modifying the mechanical properties of foods prior to consumption. Accordingly, if stone tools were used to mechanically alter fracture resistant foods, we predict that they should not be expected to be used by megadont hominins (the ownership of stone tools is often uncertain [33]), and that this is due to differences in potential fitness gain.

\subsubsection{Informing paleo-dietary evidence}

Plants that utilize $\mathrm{C}_{3^{-}}$and $\mathrm{C}_{4}$-photosynthetic pathways have different ratios of stable carbon isotopes $\left({ }^{13} \mathrm{C} /{ }^{12} \mathrm{C}\right)$. These isotopic ratios are recorded in consumer tissues and can be used to quantify the proportions of isotopically distinct resources in a consumer's diet. Animals that consume $\mathrm{C}_{3}$-photosynthetic plants (e.g. shrubs and trees) tend to be depleted in ${ }^{13} \mathrm{C}$ (high $\delta^{13} \mathrm{C}$ values), while those consuming $\mathrm{C}_{4}$-photosynthetic plants (e.g. tropical grasses and sedges) tend to be enriched in ${ }^{13} \mathrm{C}$ (high $\delta^{13} \mathrm{C}$ values) [85]. Here and henceforth, we refer to the $\delta^{13} \mathrm{C}$ values of carbonate (where $R={ }^{13} \mathrm{C} /{ }^{12} \mathrm{C}$

and $\delta=1000\left(\left(R_{\text {sample }} / R_{\text {standard }}\right)-1\right)$, with units of per-mil, \%o $)$, the mineral component 
of bone and tooth enamel used in most stable isotope paleodietary reconstructions. Megadont ( $P$. robustus and $P$. boisei), as well as some non-megadont hominins $(A$. africanus), have tissues that are enriched in ${ }^{13} \mathrm{C}[175,191]$, suggesting that they were consuming either large quantities of $\mathrm{C}_{4}$ plants or animals that consumed $\mathrm{C}_{4}$ plants. These findings are at odds with evidence from dental microwear, which tend to support consumption of non-fracture resistant foods $[189,190,191]$. Here we examine how the isotopic composition of simulated foragers can be influenced by energetic reserves and enamel volume over a lifespan by incorporating carbon isotope ratios as a function of foraging decisions into the forward iteration algorithm (see Appendix 4.1). Moreover, we show that the disparity between patterns of molar microwear and isotopic ratios can be explained by the foraging decisions predicted by our model.

Enamel volume affects optimal foraging decisions, and these factors were found to influence the modeled $\delta^{13} \mathrm{C}$ values of a simulated population of $N=100,50 \mathrm{~kg}$ anthropoid foragers capable of mechanically altering both arthropods and USOs. In dry environments (where food is more likely to be poor quality) and when foraging costs are minimal $\left(c_{i}=C_{1} \cdot \mathrm{RMR} \cdot\left(1 / \mu_{i}\right) / 2388\right)$, the mean $\delta^{13} \mathrm{C}$ value was relatively high $(\approx-10.5 \%$; Fig. $4.5 \mathrm{~A})$, due to a greater reliance on USOs. As energetic reserves varied and enamel volume decreased over time, the optimal foraging choices changed in accordance to the decision matrix (Fig. S4.2; $x_{\max }=50$ ). After day 3500 the mean $\delta^{13} \mathrm{C}$ value decreased to $\approx-11.2 \%$ as the proportional contribution of USOs decreased and that of fruits increased (Fig. 4.5B). This highlights the increasing importance of foods that are less fracture resistant as enamel is worn, as well as the accompanying 
decrease in $\delta^{13} \mathrm{C}$ of the consumer over its lifespan.

By contrast, in environments where foraging costs are maximized $\left(c_{i}=\left(C_{1}\right.\right.$. $\left.\left.\mathrm{RMR} \cdot\left(1 / \mu_{i}\right)+C_{2} \cdot \mathrm{RMR}\right) / 2388\right)$ and the risk of starvation is increased, high calorie, non-fracture resistant foods are chosen less despite greater enamel wear. This results in a relatively higher mean $\delta^{13} \mathrm{C}$ value $(\approx-8.8 \%$ ) with greater variance (Fig. $4.5 \mathrm{C})$. These simulated $\delta^{13} \mathrm{C}$ values are similar to those observed for Australopithecus africanus and Paranthopus robustus [191]. In high-cost environments, USOs tend to represent the optimal food choice (Fig. 4.5D) until near $t_{\max }$, when the risk of foraging less fracture resistant but rare foods is superseded by declining enamel volume, observed by a decrease in the mean $\delta^{13} \mathrm{C}$ value. These results help resolve the observed discrepancy between hominin isotopic ratios and molar microwear. Molar enamel is formed early in life [104], and food selection during this time tends towards fracture resistant foods with high $\delta^{13} \mathrm{C}$ values. As enamel is worn, optimal foraging decisions are altered, such that softer, though less abundant, foods with lower $\delta^{13} \mathrm{C}$ values are chosen (Fig. 4.5A,B). Molar microwear is formed later in life, and our model results suggest that less-fracture resistant foods should have greater influence over microwear as organism's age.

Although predicted optimal foraging decisions can result in carbon isotope ratios similar to those observed in fossil hominins, simulated megadont isotope ratios do not result in higher $\delta^{13} \mathrm{C}$ values than those for non-megadonts (Fig. S4.3). This is significant because hominins such as $P$. boisei achieved $\delta^{13} \mathrm{C}$ values $\approx 0 \%$. The inability of our model to predict such values is due to the fact that ${ }^{13} \mathrm{C}$-enriched foods such as grass leaves are not solutions to the SDP for either non-megadonts or megadonts (Fig. 
4.3). However, because robust megadonts such as $P$. boisei have diets composed of ca. 75-80\% $\mathrm{C}_{4}$-photosynthetic plants [191], we can explore under what parameterizations foods such as $\mathrm{C}_{4}$-photosynthetic grass leaves are chosen. We find that when fracture resistant, low risk, and low calorie foods are hyper-abundant, they become represented in both non-megadont and megadont decision matrices (Fig. 4.6A,B). When grass leaves are hyper-abundant, they are selected as optimal foods when enamel volume is high, and energy reserves are low. Moreover, a megadont dentition leads to a greater number of SDP solutions identifying grass leaves as optimal foods (Fig. 4.6B), compared to non-megadonts. Thus, our model predicts that greater enamel volume leads to a higher probability that fracture resistant, nutritionally poor $\mathrm{C}_{4}$-photosynthetic foods are optimal foraging solutions, but only when these foods are hyper-abundant.

\subsubsection{Updating dietary information with process-based models}

We have used an SDP-based approach to predict the diets of anthropoid foragers as a function of energy reserves and enamel wear, however the same approach can be used to update knowledge of trophic interactions, particularly when isotopic data is the primary means of inference. Because dietary knowledge of past organisms is often based on limited information with relatively few observations and large uncertainties, the problem is well-suited to inference using Bayesian methods. Bayesian isotope mixing models are recent tools used to quantify the proportional contribution that different resources contribute to the isotopic composition of a consumer [122]. Such tools are of

greatest utility when more than one isotopic tracer is used (e.g. carbon and nitrogen), 
though this potential is often limited in deep-time paleontological contexts. Despite this shortcoming, a Bayesian approach can integrate knowledge of uncertainties regarding dietary estimates from different approaches in an iterative fashion, such that current estimates of diet (the prior) can be used to inform the next set of dietary data once it is available [70]. With respect to using stable isotopes to determine diet, the technological advancement of tools used to analyze these data has outpaced the ability to construct informative priors [206]. We suggest that process-based models can be used to build dietary priors thereby directly informing independent estimates of diet, and in particular, used with stable isotope mixing models.

Stochastic Dynamic Programs are useful for building informative priors because dynamic processes are stated explicitly and related directly to both physiological and environmental constraints, as well as the underlying stochasticities. Estimating the magnitude and directionality of effects on foraging decisions imposed by such constraints is vital for both reconstructing and interpreting dietary evidence, particularly for extinct organisms. The dietary choices of simulated foragers can be expressed as a prior distribution, as observed in Fig. 4.5B,D (here, the proportional contribution estimates can be used to fit a Dirichlet, or multivariate Beta, distribution - commonly used as a prior distribution in isotope mixing models). In both cases (the low-cost and high-cost scenario), the proportional contribution of food resources to forager diets differs strongly from the mean encounter rates of the different food-items. This illustrates the importance of considering the processes outlined above, and the degree to which diet can be altered, when reconstructing the dietary habits of foragers. Moreover, in 
cases where different paleo-dietary tools reflect diet across multiple life-history stages, different prior distributions can be used to more accurately incorporate time-dependent behaviors (Fig. 4.5B: I,II).

\subsubsection{Conclusions}

Foraging decisions reflect the cumulative demand of many probabilistic events, particularly those that impact nonrenewable resources used to procure or consume food [80]. Understanding the relationships between these resources and the resultant optimal foraging decisions using process-based models provides a baseline by which an organism's diet can be predicted. Because nonrenewable resources such as molar enamel volume vary strongly among closely related hominin species, process-based models provide a means by which the fitness benefits of thick enamel can be quantified. Integrating modelbased approaches with dietary data - collected from multiple lines of inquiry - will not only provide a means by which uncertainty is accurately estimated, but may facilitate a deeper understanding of the processes that contribute to diet choice, particularly for extinct organisms.

\section{Acknowledgements}

We thank CE Chow, AM Kilpatrick, TS Kraft, T Levi, PW Lucas, AD Melin, GL Moritz, M Novak, AO Shelton, and ER Vogel for insightful comments and helpful discussions. This work was partially supported by National Science Foundation (NSF) Grant 2009-0417 to MM, and a NSF graduate research fellowship to JDY. 


\subsection{Tables}

\begin{tabular}{llll}
\hline Parameter & Interpretation & $\begin{array}{l}\text { Value(s) } \\
\text { Rich habitat quality }(\mathrm{r})\end{array}$ & Poor habitat quality $(\mathrm{p})$ \\
\hline$X(t)$ & Energy reserves at time $t$ & State Variable & \\
$V(t)$ & Enamel volume at time $t$ & State Variable & \\
$K$ & Number of food items found & Stochastic Variable & \\
$\Omega$ & Basal enamel wear & Stochastic Variable & $(1.4,0.3,1.4,2.9)$ \\
$\gamma$ & Gain & $(1.5,0.3,1.6,3.2)$ & $(1.1,0.5,0.7,2.2)$ \\
$c$ & Cost (minimal) & $(0.7,0.5,0.7,2.2)$ & $(1.8,1.2,1.4,2.8)$ \\
& Cost (maximal) & $(1.4,1.2,1.4,2.8)$ & $(2,4,3,1)$ \\
$\xi$ & Mean encounter rate & $(3,4,3,1)$ & \\
$\nu$ & Dispersion & $(3,5,3,2)$ & \\
$\eta$ & Digestibility & $(0.9,0.7,0.8,0.9)$ & \\
$A$ & Molar surface area & $\sum_{m=1}^{3} \pi L_{m}^{2}$ & \\
$b$ & Slope of enamel wear & 0.0425 & \\
$E$ & Young's modulus & $(1,11,5,200)$ & \\
$R$ & Fracture toughness & $(565,300,265,1345)$ & \\
$d$ & Prob. of death at time $t$ & $4.54 \times 10^{-5}$ & \\
\hline $\boldsymbol{\rho}$ & Transition probability & Wet: $(0.8,0.2 ; 0.2,0.8)$ & \\
& matrix: $\left(\rho_{\mathrm{rr}}, \rho_{\mathrm{rp}} ; \rho_{\mathrm{pr}}, \rho_{\mathrm{pp}}\right)$ & Dry: $(0.2,0.8 ; 0.8,0.2)$ & \\
\hline
\end{tabular}

Table 4.1: Parameters, interpretations, and values in the dynamic state variable model. Parenthetical values (except for $\boldsymbol{\rho}$ ) refer to those for foods: (fruit, grass leaves, USOs, arthropods). Values for $E$ and $R$ are those when no mechanical advantage is included. See methods for relevant references. 


\subsection{Figures}

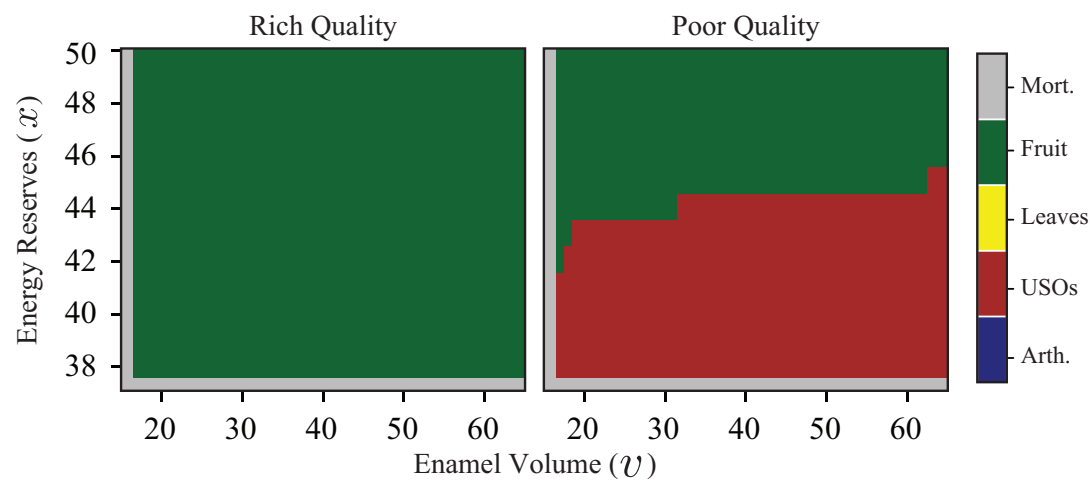

Figure 4.1: Stationary solutions to the fitness-maximizing equations $F_{\mathrm{r}, \mathrm{p}}(x, v)$ for a 50 $\mathrm{kg}$ anthropoid primate with no mechanical advantages. In rich quality habitats, fruit (green) is chosen independent of energy reserves, $x$, or enamel volume, $v$. In poor quality habitats, plant USOs (red) are chosen when energy reserves are low, and are replaced by less fracture resistant fruits as enamel volume decreases. There are no differences between wet (where the environment is primarily rich quality) or dry (where the environment is primarily poor quality) conditions. Gray elements denote values of $(x, v)$ resulting in mortality. 


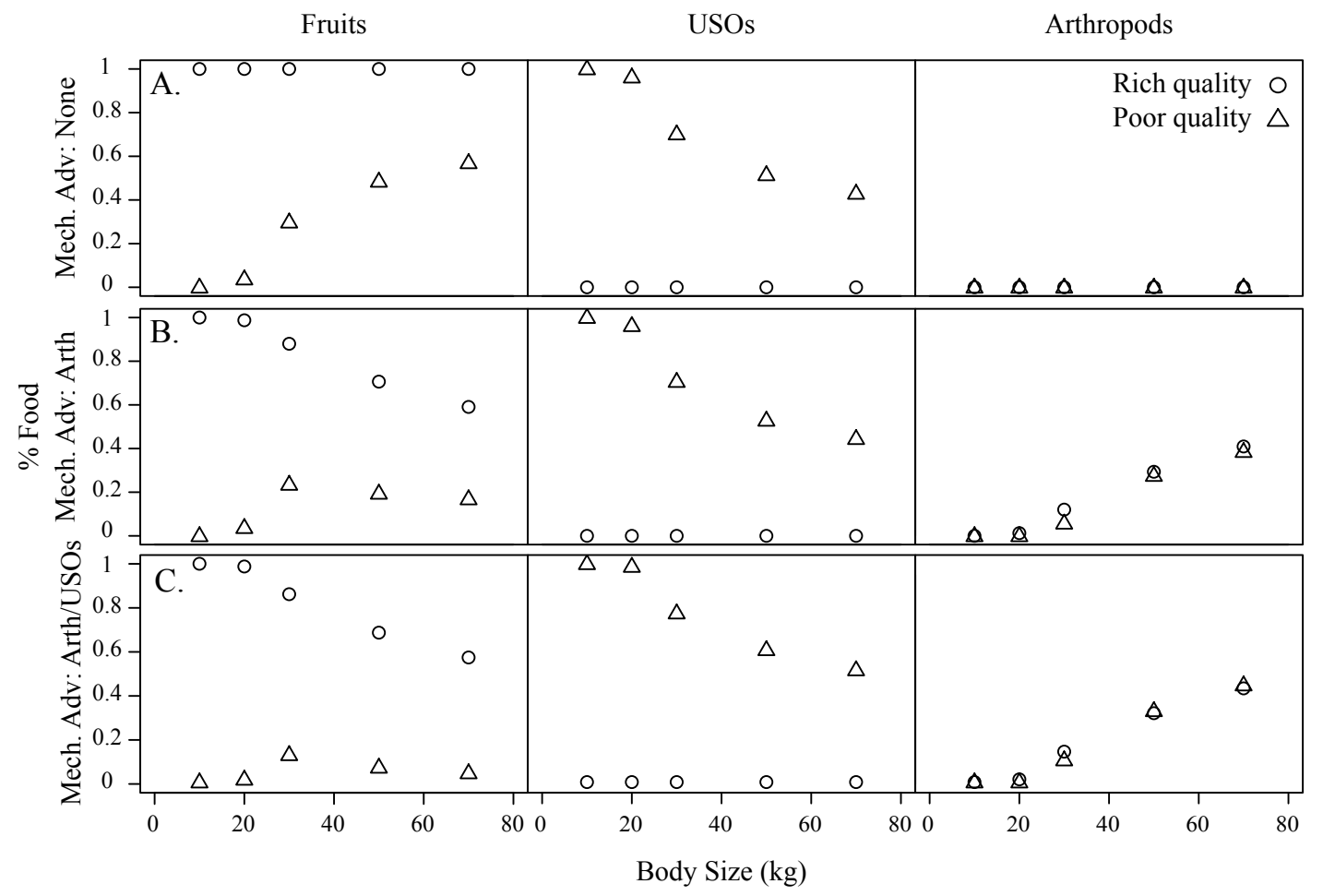

Figure 4.2: The proportional contribution of foods to the decision matrices of anthropoid primates with body sizes ranging from 10 to $70 \mathrm{~kg}$. A. Contributions of foods for the no mechanical advantage scenario. B. Contributions of foods for the arthropod mechanical advantage scenario. C. Contributions of foods for the arthropod + USO mechanical advantage scenario. Circles denote values in rich quality habitats; triangles denote values in poor quality habitats. Grass leaves are not found to be optimal foraging solutions in any decision matrix. 
1. Mechanical Advantage: None

2. Mechanical Advantage: Arthropods

3. Mechanical Advantage: Arthropods + USOs

non-Megadont

Megadont

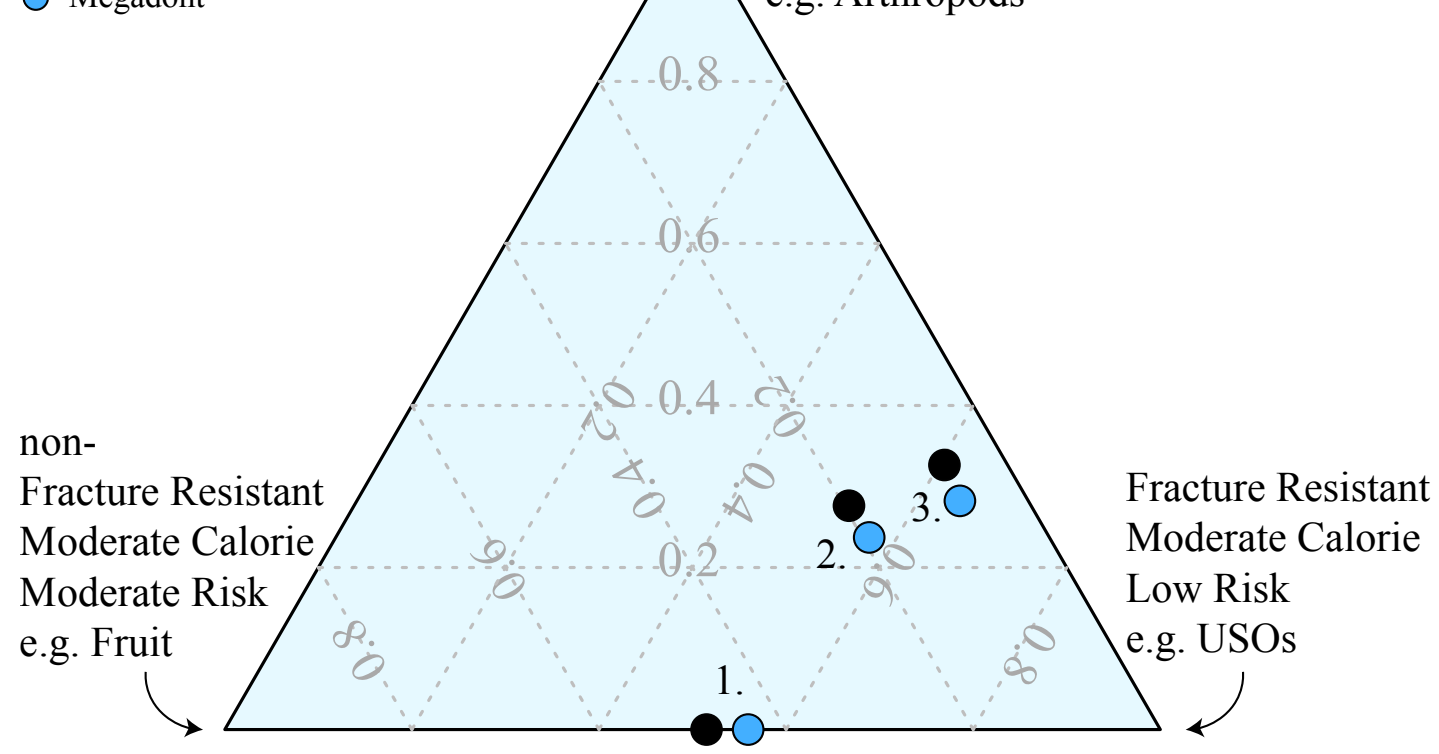

Figure 4.3: Ternary diagram showing the proportional contribution of fruit, USOs, and arthropods to the decision matrices of both $50 \mathrm{~kg}$ non-megadont and megadont primates under each mechanical advantage scenario. Megadont primates have decision matrices with a greater proportion of fracture-resistant, moderate calories, and low cost foods. 


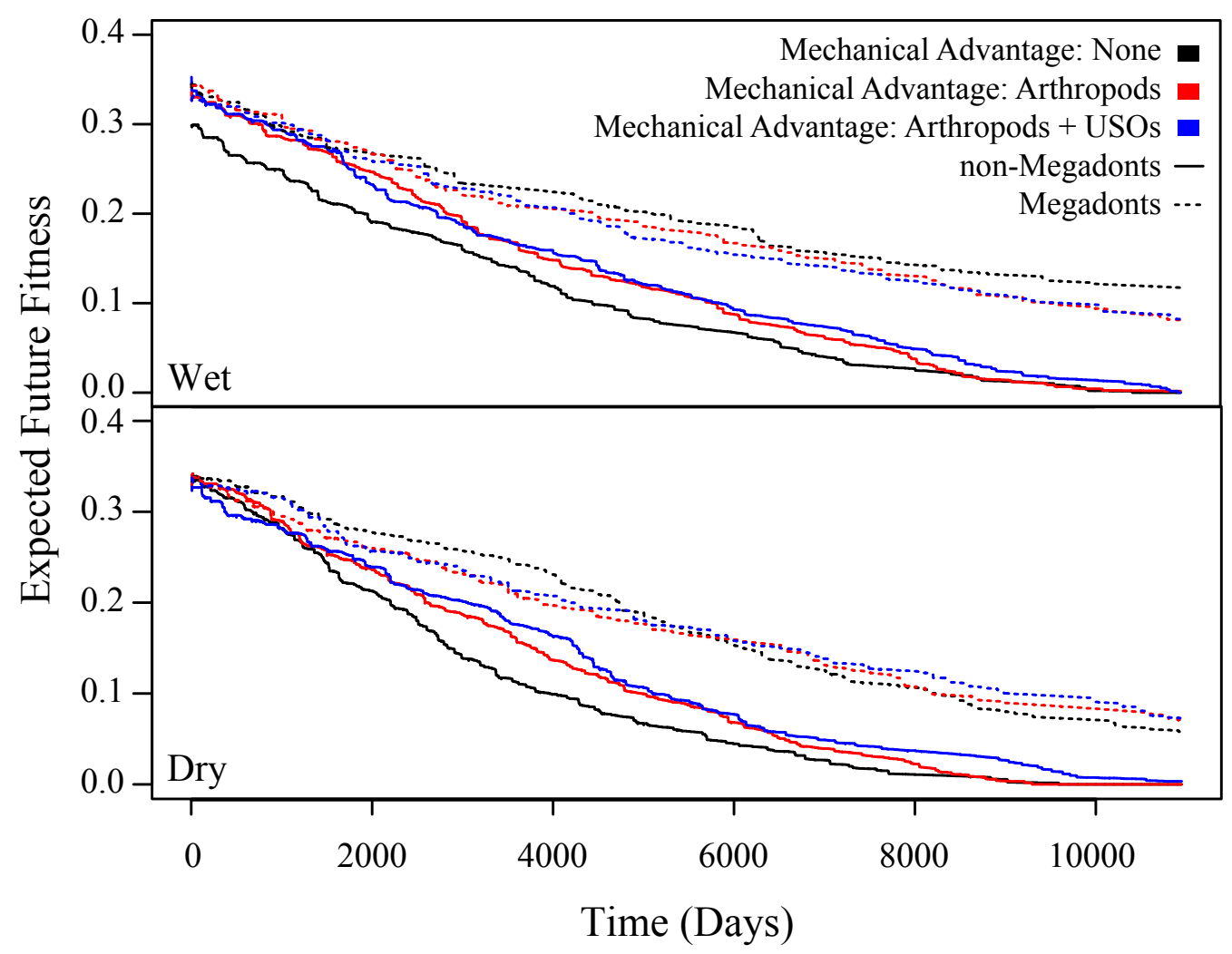

Figure 4.4: Expected fitness trajectories for $N=10050 \mathrm{~kg}$ non-megadont (solid) and megadont (stippled) primates over an estimated lifespan with varying mechanical advantages (none, arthropods, arthropods + USOs), during both wet (primarily rich quality habitat) and dry (primarily poor quality habitat) conditions. 

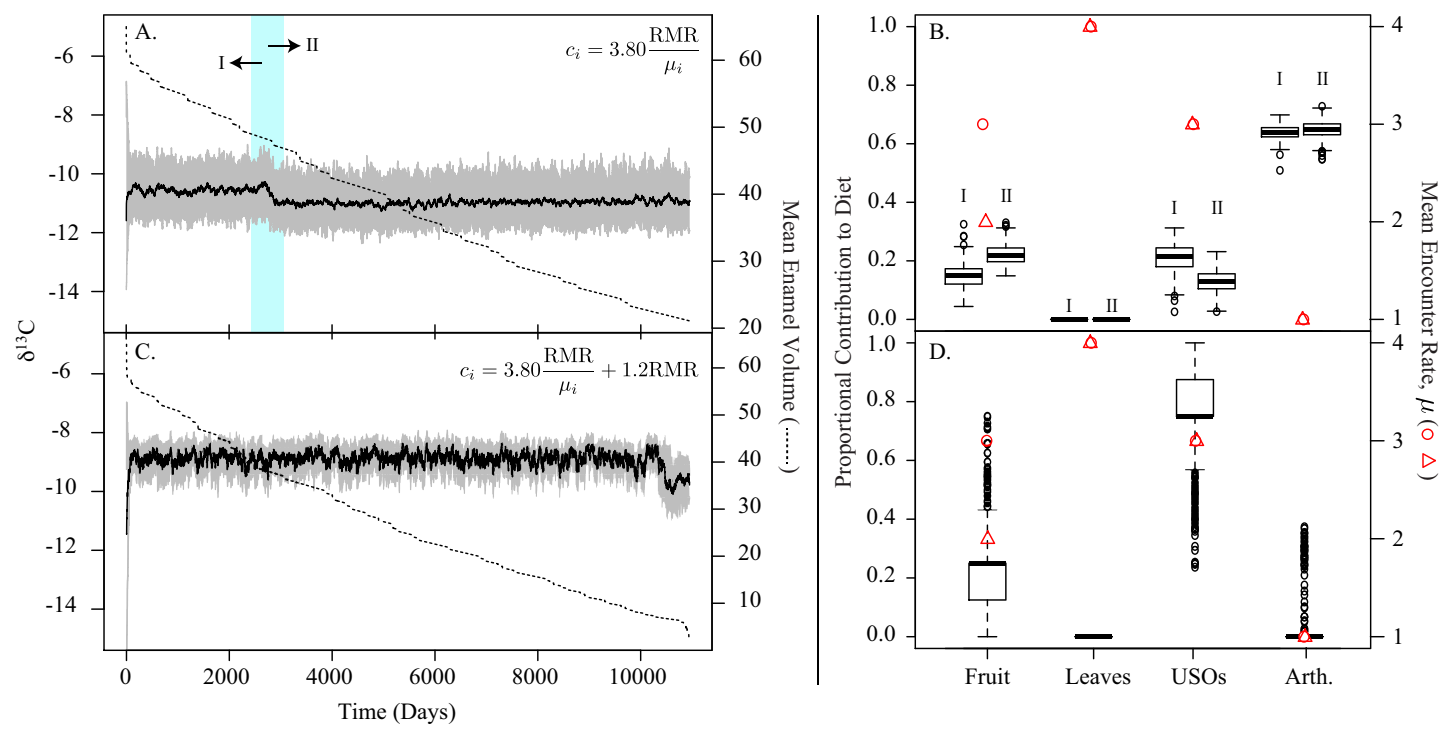

Figure 4.5: Forward simulation of the $\delta^{13} \mathrm{C}$ values (black line denotes running mean; gray band denotes maximum and minimum value), mean enamel volume, and the proportional contribution of food-items to the diets of $N=100,50 \mathrm{~kg}$ individuals foraging in a dry environment over an estimated lifespan. A. and B. When foraging costs are minimal, a dietary switch is observed to occur near day 3500, and labels I and II denote the pre- and post-diet switch. C. and D. The same simulation when foraging costs are elevated. In panels B. and D., the red circles and triangles denote the mean encounter rate for each food in rich quality and poor quality habitats, respectively. 


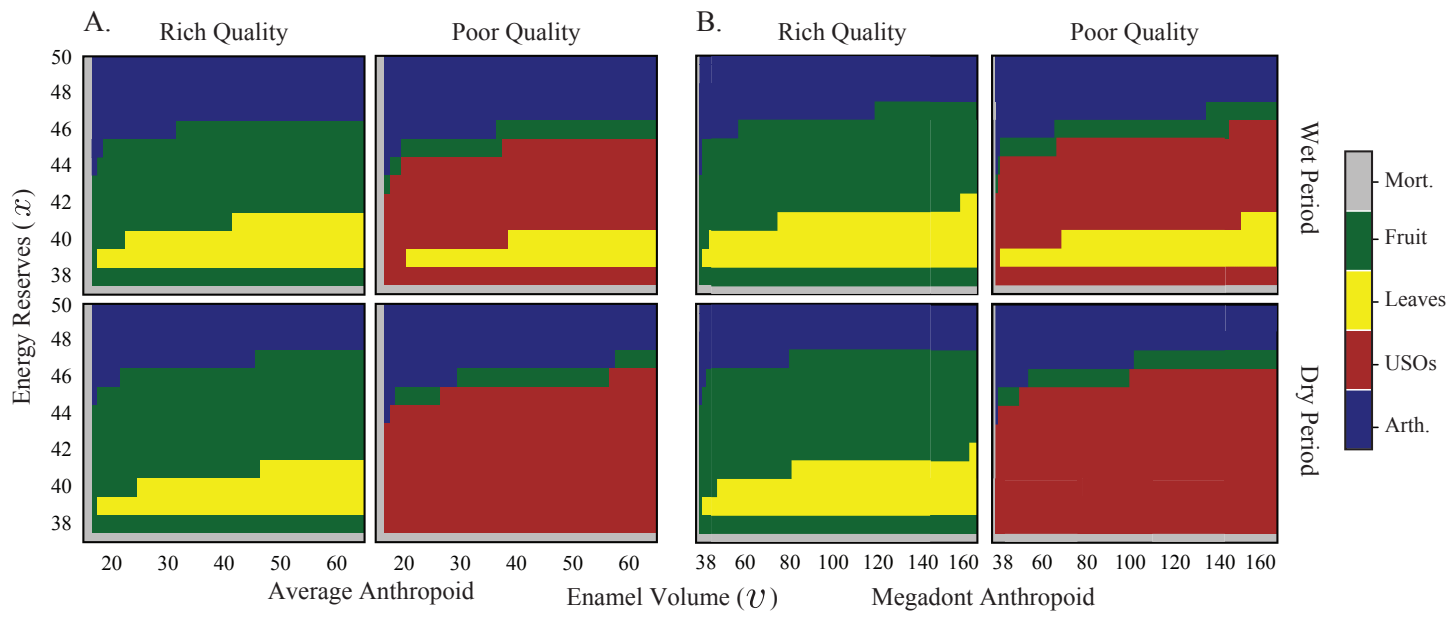

Figure 4.6: Stationary solutions for the fitness-maximizing equations $F_{\mathrm{r}, \mathrm{p}}(x, v)$ as a function of energy reserves $x$ and enamel volume $v$ for both non-megadont and megadont primates when grass leaves are hyper-abundant and for the arthropod + USO mechanical advantage scenario. Decision matrices are shown for both wet and dry environmental conditions. 


\subsection{Supplementary Material}

\subsubsection{Appendix 4.1. Modeling $\delta^{13} \mathrm{C}$ values of simulated foragers}

We simulated the time evolution of a forager's $\delta^{13} \mathrm{C}$ values as a function of predicted foraging decisions. Here and henceforth, we refer to the $\delta^{13} \mathrm{C}$ values of carbonate (where $\delta=1000\left(\left(R_{\text {sample }} / R_{\text {standard }}\right)-1\right.$ ), and $R={ }^{13} \mathrm{C} /{ }^{12} \mathrm{C}$, with units of per-mil, \%o), the mineral component of bone and tooth enamel used in most stable isotope paleodietary reconstructions. The evolution of a forager's $\delta^{13} \mathrm{C}$ values from time $t$ to $t+1$ is a function of its food choice given the forager's current internal state $(x, v)$, the quantity of found food, $K$, and the weighted mean of these foods against its current size, $X(t)$, and isotopic composition, $\delta^{13} \mathrm{C}_{n}(t)$, where $n$ denotes the $n^{\text {th }}$ individual of a population of size $N$. The change in the isotopic composition of the $n^{\text {th }}$ forager is given by the difference equation

$$
\delta^{13} \mathrm{C}_{n}(t+1)=\frac{X(t) \cdot \delta^{13} \mathrm{C}_{n}(t)+K_{i} \cdot\left\langle\delta^{13} \mathrm{C}_{i}\right\rangle}{X(t)+K_{i}}
$$

where $\delta^{13} \mathrm{C}_{n}(t)$ denotes the isotopic composition of the $n^{\text {th }}$ forager at time $t$, and $\left\langle\delta^{13} \mathrm{C}_{i}\right\rangle$ denotes the mean value of $K$ items of food $i$.

The $\delta^{13} \mathrm{C}$ values of the potential food choices were modeled by randomly drawing values from the $\delta^{13} \mathrm{C}$ distributions of animals known to consume browse (to model the isotopic composition of a fruit diet; mean \pm a standard deviation $=-11.5 \pm 1.3 \%$ ), graze (to model the isotopic composition a grass leaf diet; $0 \pm 1 \%$ ), and USOs (averaged values for the enamel and dentine of Pliocene Cryptomys mole rats; $-8.21 \pm 1.32 \%$ ) in 
African savanna-woodland environments $[176,204]$. Termites in mixed $\mathrm{C}_{3} / \mathrm{C}_{4}$ environments [176] were used to model the $\delta^{13} \mathrm{C}$ values of an arthropod diet, and an adjustment of $+9 \%$ was applied to account for the metabolic fractionation between termites and a hind-gut fermenting consumer $(-12 \pm 2.1 \%$ ). 


\subsubsection{Figures}
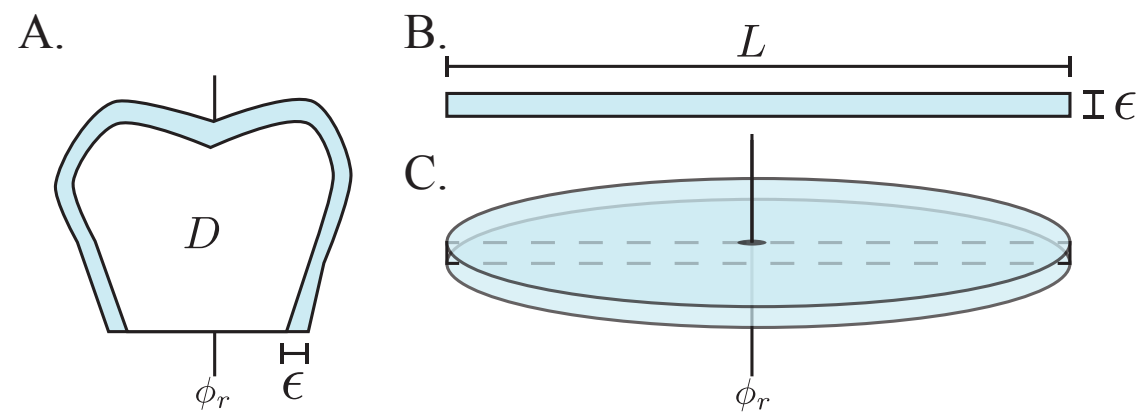

Supplementary Figure 4.1: A. A cross-section of a molar. The enamel coat and dentin are colored blue and white, respectively. The average enamel thickness, $\epsilon$, is given by the average width of the enamel coat, and the dentin area, $D$, is measured by the area of dentin observed under the cross-section. B. To calculate the enamel volume of the molar, we first simplify the complex topology of the enamel coat by modeling its cross-section as a rectangle with a length equal to that of the enamel-dentin junction, $L$, and height equal to the average enamel thickness, $\epsilon$. C. Volume is calculated by modeling the enamel cross-section as a 'solid of revolution'. Total enamel volume is measured by calculating the volume of the cylinder produced when rotating the crosssection about the axis of rotation, $\phi_{r}$. This relies on the assumption that the tooth is fairly symmetrical about the axis of rotation. 


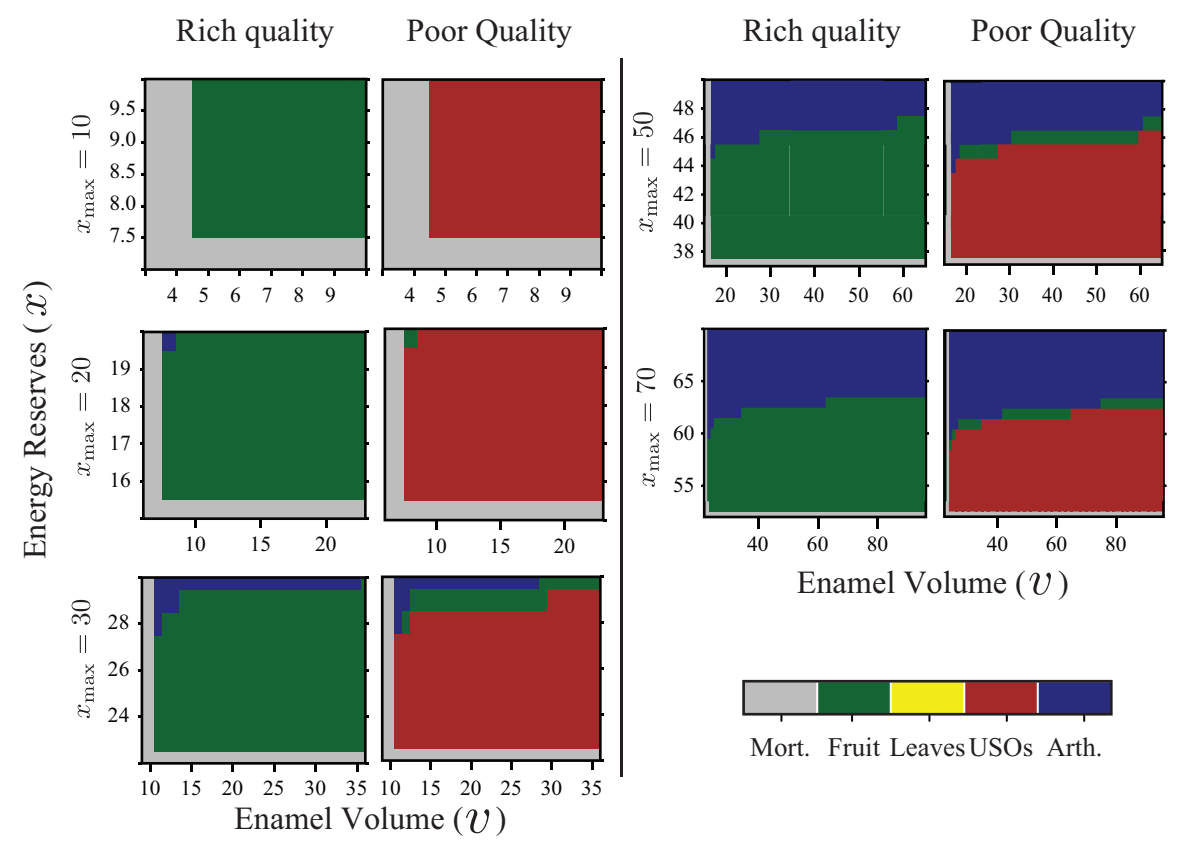

Supplementary Figure 4.2: Stationary solutions for the fitness-maximizing equations $F_{\mathrm{r}, \mathrm{p}}(x, v)$ as a function of energy reserves $x$ and enamel volume $v$. Decision matrices are shown for the arthropod + USO mechanical advantage scenario. Each pair of decision matrices represent an anthropoid primate with a body size ranging from $x_{\max }=10$ $\mathrm{kg}$ to $70 \mathrm{~kg}$. There are no differences between wet (primarily rich quality habitat) or dry (primarily poor quality habitat) conditions. Gray elements denote values of $(x, v)$ resulting in mortality. 


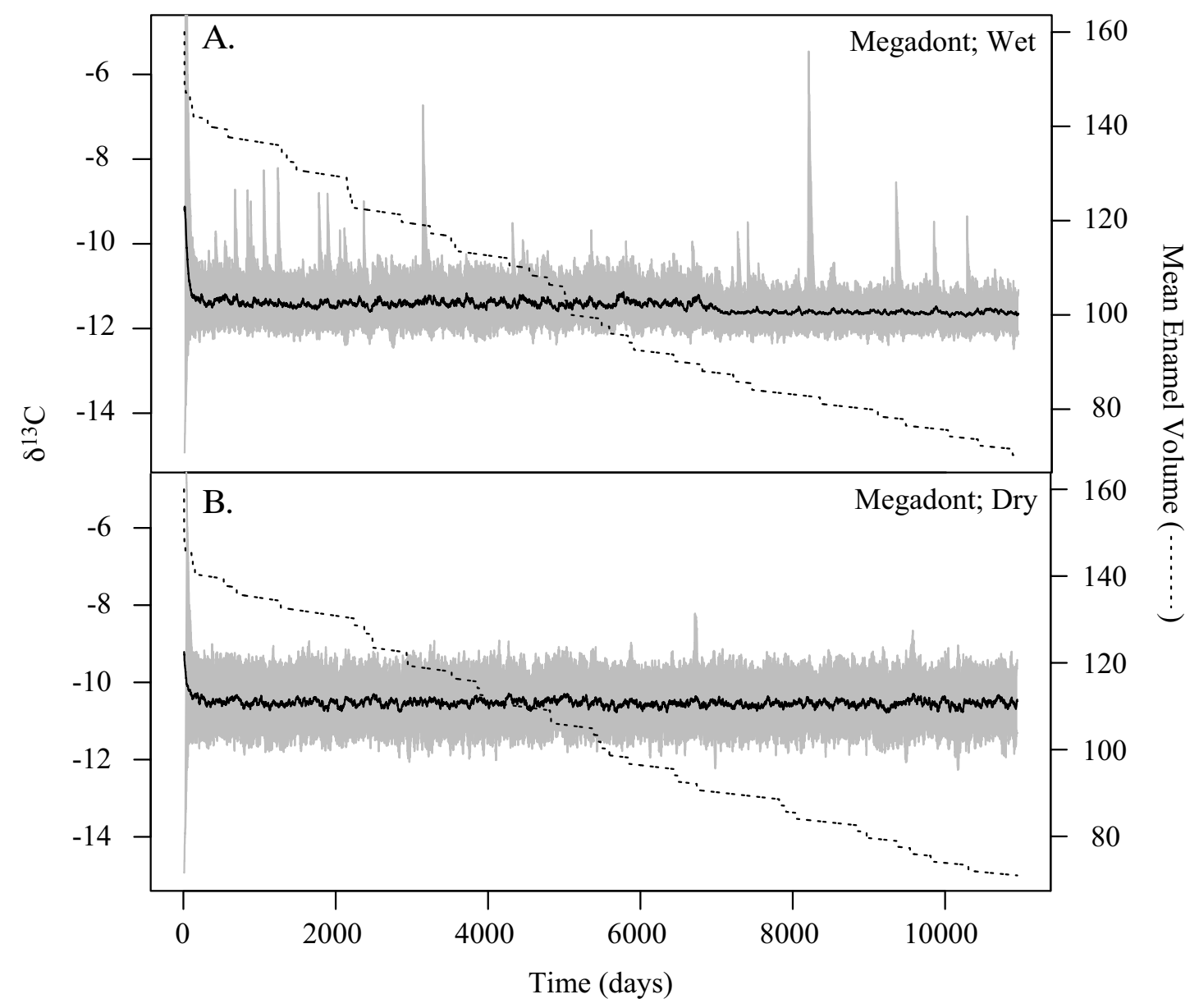

Supplementary Figure 4.3: Forward simulation of the $\delta^{13} \mathrm{C}$ values (black line denotes running mean; gray band denotes maximum and minimum value), mean enamel volume, and the proportional contribution of food-items to the diets of $N=100,50 \mathrm{~kg}$ megadont hominins foraging in wet and dry environments over an estimated lifespan. 


\section{Chapter 5}

Merging resource availability with

isotope mixing models: The role of

\section{neutral interaction assumptions}

J D Yeakel, M Novak, P R Guimarães Jr., N J Dominy, P L Koch, E J Ward, J W Moore, B X Semmens 


\section{Abstract}

Bayesian mixing models have allowed for the inclusion of uncertainty and prior information in the analysis of trophic interactions using stable isotopes. Formulating prior distributions is relatively straightforward when incorporating dietary data. However, the use of data that are related, but not directly proportional, to diet (such as prey availability data) is often problematic because such information is not necessarily predictive of diet, and the information required to build a reliable prior distribution for all prey species is often unavailable. Omitting prey availability data impacts the estimation of a predator's diet and introduces the strong assumption of consumer ultrageneralism (where all prey are consumed in equal proportions), particularly when multiple prey have similar isotope values. We develop a procedure to incorporate prey availability data into Bayesian mixing models conditional on the similarity of isotope values between two prey. If a pair of prey have similar isotope values (resulting in highly uncertain mixing model results), our model increases the weight of availability data in estimating the contribution of prey to a predator's diet. We test the utility of this method in an intertidal community against independently measured feeding rates. Our results indicate that our weighting procedure increases the accuracy by which consumer diets can be inferred in situations where multiple prey have similar isotope values. This suggests that the exchange of formalism for predictive power is merited, particularly when the relationship between prey availability and a predator's diet cannot be assumed for all species in a system. 


\section{$5.1 \quad$ Introduction}

\subsubsection{Background}

Trophic interactions are fundamental components of biodiversity, directly contributing to ecosystem organization and dynamics [171]. Accurate reconstruction and quantification of the strengths of trophic interactions remains a challenge in most ecological systems [138]. Often, it is impossible to directly observe or document feeding relationships [198]. In many such cases, ratios of stable isotopes (typically those of carbon: ${ }^{13} \mathrm{C}:{ }^{12} \mathrm{C}$ and nitrogen: ${ }^{15} \mathrm{~N}:{ }^{14} \mathrm{~N}$ ) can be used to investigate the diets of consumers [85]. Carbon isotope ratios distinguish primary producers that use different photosynthetic pathways (or that differ in other physiological or physiognomic attributes) and are conserved in the tissues of consumers, whereas ratios of nitrogen isotopes are strongly sensitive to trophic level (though they may vary spatially or with primary producer functional type). Accordingly, stable isotope data of both consumer and potential prey can be used to quantify a consumer's resource niche, can provide dietary information relevant across a range of temporal and spatial scales, and can distinguish dietary differences across hierarchies of animal communities [49, 128, 163, 207].

The extent to which inference can be successfully drawn from stable isotope data is dependent upon the isotopic uniqueness of a consumer's prey (here prey' may refer to any resource that is consumed by an organism). When different potential prey are isotopically distinct and the number of prey are greater than the number of isotopic tracers +1 , analytical mixing models may be employed to assess the possible 
contributions of prey to a consumer's diet $[101,144,145,146]$. Because many different combinations of dietary sources can produce a given set of isotope values under these conditions, quantitative tools are required to parse less likely trophic interactions from those that are more likely to occur. Bayesian mixing models can be used to numerically simulate posterior probability distributions that quantify the range and likelihood of all potential source combinations $[163,122,139]$. This approach requires prior knowledge of a consumer's diet to be designated, even if that knowledge is specified to be uninformative (i.e. that each prey item has an equal probability of contributing to the predator's diet). In addition, all isotopic mixing models require the accurate specification of prey isotope values and trophic fractionation factors [29].

Despite recent advances in Bayesian mixing models, estimates of dietary relationships may be inaccurate or uninformative when multiple prey are isotopically similar (this issue is problematic in non-Bayesian mixing models as well) [145]. Here we address the issue of isotopic similarity among prey that are not equally available to a consumer. Because the relative availability of a specific prey will theoretically have a direct impact on the consumer's diet, we propose a method to employ data reflecting these differences in availability to weight the probability distributions of a consumer's reliance on a particular prey. Because the precise relationship between a prey's inclusion to a predator's diet and a prey's availability is uncertain (and is not necessarily applicable to all prey in a predator's diet), we condition the influence of availability data on the isotopic uniqueness of a given prey. 


\subsubsection{Incorporating biological information}

The quantification of a consumer's diet implicitly assumes that all dietary resources included in an analysis are potentially important. If an uninformative prior is used in a Bayesian mixing model, all prey are assumed to contribute equally to a predator's diet a priori. This specification allows isotopic data to maximally influence the results of the mixing model. Thus, in a system with $n$ potential prey sources, the a priori assumption is that the consumer is an ultrageneralist, consuming a unit of biomass

of prey $i$ with the probability: $c_{i}=n^{-1}$. The unique isotopic distribution of prey relative to the consumer's isotope values can modify this probability. Nevertheless, the a priori assumption will be especially relevant when two prey occupy the same isotopic space. Here, the model will predict that isotopically similar prey contribute equally to a consumer's diet.

Additional biological information regarding prey sources may render such generalizations over-simplistic; if the relative availability of prey on the landscape is known $\left(\alpha_{1}, \alpha_{2},, \alpha_{n}\right.$; where the sum of these elements is equal to unity), the underlying assumption may be revised to reflect these differences such that the probability of consuming a prey source is $c_{i}=\alpha_{i}$. Accordingly, the system operates under conditions imposed by interaction neutrality (consumption in proportion to prey abundance) [74, 92], as opposed to ultrageneralism (consumption in proportion to the number of prey available to the consumer).

In theory, prey availability data, as quantified by abundance, biomass, con- 
sumer preference, or a combination thereof, can be used to inform dietary relationships within the framework of traditional Bayesian mixing models. We acknowledge upfront that, like all aspects of isotopic mixing models, such a practice should be used with caution. Specifically, there exists an implicit assumption that prey availability and consumer-prey interactions are directly related for every prey item that is included in

the analysis. Importantly, the calculation of a consumer's diet based on prey availability and/or isotopic data are not equivalently informative. Isotopic data of a consumer and its potential prey are empirical measurements that track the flow of matter within a physical system. The relationship between isotopic data and trophic interactions has been well established in many ecosystems [85, 128, 60, 84]. On the other hand, the relationship between prey availability and diet choice is still not well understood [1], need not be system-specific [77], and may differ across prey, even for the same predator [77]. In these scenarios, prey availability data should not be introduced indiscriminately to inform Bayesian mixing models. Additionally, the use of prior information in Bayesian mixing models is subject to a number of important constraints, such that incorporation of biological information by means other than the Bayesian paradigm may be advantageous, depending on what data are available for a particular system.

\subsubsection{Post-hoc adjustments vs. the formulation of prior distributions}

Bayesian isotope mixing models allow researchers to apply prior information to an isotopic system, thereby making use of different sources of biological data that provide information regarding a consumer's diet. Prior distributions represent the a 
priori knowledge of a consumer-resource relationship, and can be parameterized by direct or indirect measurements of prey consumption by a predator. The effect that a prior distribution has, relative to the likelihood, is partially dependent upon the uncertainty, or variance, of the prior distribution [62]. The formulation of informative priors is based on two logical assumptions. First, the Bayesian algorithm assumes that the data used to formulate the prior and likelihood are determinants of the same variable (in this case, diet). Second, the parameterization of prior probability distributions is contingent on calculable metrics, such as the mean and variance.

There are scenarios where one or both of these assumptions cannot be met. Regarding the first assumption, an example involving the use of gut contents data to formulate a prior for an isotope mixing model is presented in Moore and Semmens [122]. Because both gut contents data and the isotope values of a system are indirect measures of $\operatorname{diet}(\theta)$, they lend themselves to a Bayesian framework wherein the posterior probability $p(\theta \mid y)$ is informed by both the prior $p(\theta)$ and the likelihood $p(y \mid \theta)$

$$
p(\theta(\mid y) \propto p(\theta) p(y \mid \theta)
$$

where $y$ represents isotopic data, and $\theta$ is a dietary parameter. According to Eq. (5.1), it is evident that $p(\theta \mid y)$ and $p(\theta)$ must both describe diet. Alternatively, if $p(\theta)$ represents measures of prey availability to a consumer and $p(y \mid \theta)$ is the likelihood shaped by isotopic (dietary) data, it is unclear what $p(\theta \mid y)$ describes, unless it is assumed that availability is directly correlated with diet. Prey availability would be an indirect measure of diet if species interactions follow neutral dynamics [74, 92]. For predator-prey 
interactions that are influenced by non-neutral, prey-specific ecological factors such as predator preference, prey defense, or habitat variability, measurements of prey availability cannot lend themselves to indiscriminate use as prior distributions as they no longer have a one-to-one relationship with their contribution to a consumer's diet.

As importantly, the second assumption requires that prior distribution parameters be calculable. Estimates of both mean and variance must be made for all prey species in the predator's diet to formulate appropriate priors. In systems where isotopic data are likely to be of maximum utility (e.g. systems that are difficult to observe directly), such parameters are often difficult (if not impossible) to estimate well, rendering prey availability data to parameterize prior distributions unviable. Moreover, in systems where few measurements of prey availability exist, or when count (census) data are used, variance is often underestimated [168], which can result in inaccurate posterior distributions [40]. Often, prey availability is better characterized for some species than for others. Differences in body size, temporal habitat use, and many other factors may confound parameterization of availability distributions of some species, even if the same quantities are well known for others. This renders the formulation of an accurate prior distribution difficult or impossible. In order to avoid these pitfalls, and to maximize the utility of isotope mixing models, a more targeted procedure designed to inform isotope-based measurements of a consumer's diet in a post-hoc fashion is warranted.

Here we introduce a procedure that incorporates, and appropriately weights, biologically relevant information into the posterior distributions of a Bayesian isotope mixing model. With this approach, the assumption of interaction neutrality is incorpo- 
rated into mixing model results conditional on the isotopic similarity of prey sources. This condition preserves the intrinsic differences between direct measurements that describe a system (isotopic data), and independent data that may be theoretically linked to a system (prey availability data). First, we introduce the utility of this approach with a hypothetical isotopic system. We then test the effectiveness of our approach at increasing the accuracy of trophic interaction estimates by comparing isotope data and independently estimated foraging rates of a whelk predator feeding on its prey in a New Zealand intertidal community.

\subsection{Methods}

\subsubsection{Weighting of Bayesian isotope mixing models}

Bayesian isotope mixing model output is often summarized in a matrix of estimated dietary contribution vectors $\left(\rho_{i},, n\right.$; accepted results from a Markov Chain Monte Carlo, MCMC, or a Sampling-Importance-Resampling, SIR, algorithm), where each element in the vector is the contribution of a potential prey source, and each proposed vector sums to unity [122]. Proportional prey availability can be incorporated into the vector that defines the contribution of dietary sources for a particular consumer by multiplication and renormalization. Because direct physical measurements of trophic relationships (e.g. isotopic data) are likely to be more informative than the idealized assumption of a relationship between prey availability and diet, we allow prey availability

data to influence dietary contribution results only in proportion to the extent that the 
prey have similar isotope values. As such, our model allows availability data to influence the source contribution vector in proportion to the pairwise isotopic overlap between prey. Isotopic overlap is proportional to the probability that the isotopic values of two sources are misidentified. Relative to a single pair of potential prey within a larger system, we define the impact of prey availability and isotopic data on final contributionto-diet values to be exactly inversely proportional: when there is no overlap of prey sources, isotopic data are singularly informative; when there is complete overlap of prey sources, availability data are singularly informative (for the prey pair in question). We utilize Pianka's measure of density overlap to estimate the degree of isotopic overlap between two prey sources with normally distributed isotope values. Pianka's measure of overlap $\left(w_{i j}\right)$ is defined by

$$
w_{i j}=\frac{\int g_{i}(x) g_{j}(x) d x}{\sqrt{\int g_{i}^{2}(x) d x} \sqrt{\int g_{j}^{2}(x) d x}}
$$

where $g_{i, j}(x)$ represent multivariate normal distributions of isotope values for overlapping prey sources $i$ and $j$ [147]. Solving Eq. (5.2) with the assumption of bivariate normality yields

$$
w_{i j}=\frac{\sqrt[4]{\left|\Sigma_{i} \Sigma_{j}\right|}}{\sqrt{\left|\frac{1}{2}\left(\Sigma_{i}+\Sigma_{j}\right)\right|}} e^{-\frac{1}{2}\left(\mu_{i}-\mu_{j}\right)^{\prime}\left(\Sigma_{i}+\Sigma_{j}\right)^{-1}\left(\mu_{i}-\mu_{j}\right)},
$$

where $\mu_{i}$ and $\mu_{j}$ are vectors of bivariate distribution means, $\Sigma_{i}$ and $\Sigma_{j}$ are covariance matrices of prey $i$ and $j$, respectively, and $\left(\mu_{i}-\mu_{j}\right)^{\prime}\left(\Sigma_{i}+\Sigma_{j}\right)^{-1}\left(\mu_{i}-\mu_{j}\right)$ is the square of the Mahalanobis Distance (a generalization of Euclidean Distance) [100]. While 
this metric is capable of handling isotopic distributions with alternative non-Gaussian covariance structures, mixing models have not yet taken isotopic covariance into account, and we will not discuss it further. The application of an alternative measure of overlap, Morisita's metric [123], provided nearly identical results (not reported).

As noted above, isotopic overlap represents the probability of mistaking one prey source for another; in systems where isotope values for prey $i$ and $j$ are completely distinct $\left(w_{i j}=0\right)$, assumptions of consumption based on prey availability have no influence, whereas isotopic data are regarded as singularly effective for determining a consumer's likely diet. If two prey have similar isotope values $\left(0<w_{i j} \leq 1\right)$, prey availability data are incorporated, in proportion to $w_{i j}$, to estimate the final contributionto-diet values. Accordingly, prey availability data are weighted to become increasingly informative as $w_{i j}$ increases. If $w_{i j}=1$ (exact isotopic overlap between two prey), isotopic and prey availability data are equally informative. We define the strength of this weighting value for prey $i$ and $j$ as

$$
\gamma_{i, j}=\left(1-w_{i j}\right) \frac{1}{2}+w_{i j} \alpha_{i, j}
$$

where $w_{i j}$ is the degree of isotopic overlap (Eqns. 5.2, 5.3), and $\alpha_{i, j}$ are proportional availability measurements of prey $i$ and $j$ (for example, if prey $i$ and $j$ had proportional biomass measurements of 0.25 and 0.75 , respectively, then $\alpha_{i}=0.25$ and $\alpha_{j}=0.75$ ). Therefore, the final weighted mixing model output is calculated as 


$$
f_{i, j}=\frac{\rho_{i, j} \gamma_{i, j} \sum_{i}^{j} \rho}{\sum_{i}^{j}(\rho \gamma)}
$$

where $\rho_{i, j}$ represents a vector of estimated contribution-to-diet values for prey $i$ and $j$ from a given iteration of the MCMC or SIR algorithm in a Bayesian isotope mixing model without including knowledge of relative availability. This process is employed iteratively across all proposed mixing model contribution-to-diet vectors, resulting in final probability distributions that are skewed towards relative availability $\left(\alpha_{i}, \alpha_{j}\right)$ for prey $i$ and $j$ in proportion to their isotopic similarity. As an alternative to a single ratio of two integers (e.g. $\alpha_{i}=0.25, \alpha_{j}=0.75$ ), relative availability can be incorporated as a probability distribution. This ability is excluded from these analyses for simplicity.

\subsubsection{Application to a New Zealand intertidal food web}

To assess the empirical power of our method, we applied mixing models with and without our weighting procedure to stable isotope data obtained from a predatorprey system of the New Zealand intertidal [129, 131]. Model results were then compared to biomass-weighted feeding rates calculated from independent observational data.

The focal predator is the gastropod whelk, Haustrum (=Lepsiella) scobina, which is common along the rocky intertidal shores of New Zealand. We established the diet of H. scobina at a focal study site (Tauranga Head, 41 46' $26^{\prime \prime}$ S, $171^{\circ} 27^{\prime}$ 20 " E) by performing systematic low-tide feeding surveys of the population in predetermined mid- and high-intertidal areas of the shore. Each encountered whelk was

counted and carefully examined to determine feeding activity. The sizes of all whelks 
and their identified prey items were measured to within $\pm 1 \mathrm{~mm}$ [130]. In total, 2668 whelks where encountered in 20 surveys performed over a 2-year period (May 2005 - July 2007). On average, $20.8 \%$ of all individuals were actively feeding such that a total of 554 feeding observations on 8 prey species were recorded. The eight observed prey species were: the snails, Austrolittorina antipodum $(A a)$, A. cincta $(A c)$, and Risellopsis varia $(R v)$; a limpet Notoacmea sp. (Nr); the mussels Mytilus galloprovincialis $(M g)$ and Xenostrobus pulex $(X p)$; and the barnacles Chamaesipho columna $(C c)$ and Epopella plicata $(E p)$ (Table 5.1). The saturated nature of the resultant species accumulation curve suggests that most of $H$. scobina's prey were documented (Fig. S5.1). Further details are provided in Novak [130].

\subsubsection{Observational estimates of prey contributions}

We used the observational method of Novak and Wootton [131] to convert observed frequencies of predation events to prey-specific estimates of $H$. scobina's feeding rates. This method required an estimation of mean prey-specific abundances and the mean prey-specific handling time required by $H$. scobina individuals to consume a prey item. Species' densities were estimated using 10-15 quadrats measuring 0.25 $\mathrm{m}^{2}$, randomly distributed across three $20-\mathrm{m}$ transects positioned within each mid- and high-intertidal zone. Abundance surveys were repeated three times (MayJuly 2005, JanuaryFebruary 2006, May 2006) such that site-wide mean prey densities were estimated on the basis of 60-90 quadrats (Table 5.1). H. scobina's handling times (the time required by an individual to drill and ingest a prey item) were measured in controlled 
laboratory experiments where prey identity and relative predator-prey body size could be manipulated independently $(\mathrm{n}=208)$. The prey-specific relationships observed between these variables allowed us to estimate the expected handling time of each feeding event observed in the field. These data were then used to calculate prey-specific mean handling times (Table 5.1). Further details are provided in Novak [130].

Data from the feeding surveys, abundance surveys, and field-estimated handlingtimes were combined to calculate prey-specific per capita attack rates $\left(c_{i}\right.$, the number of prey eaten per predator per prey available per $\mathrm{m}^{2}$ per day) as

$$
c_{i}=\frac{F_{i} A_{x}}{\left(F_{x}-A_{x}\right) h_{i} N_{i}},
$$

where $h_{i}$ and $N_{i}$ respectively denote the $i^{\text {th }}$ prey's mean handling time (days) and mean density $\left(\# \cdot \mathrm{m}^{-2}\right), A_{x}$ denotes the proportion of individuals in the predator population (feeding and non-feeding) observed to be feeding on prey species $x$, and $F_{i}$ denotes the proportion of the population's feeding individuals observed to be consuming the $1^{\text {th }}$ prey species [131]. Species $x$ is an arbitrarily chosen species used throughout the calculation of all prey-specific attack rates [131]. Prey-specific feeding rates $\left(C_{i}\right.$, the grams of prey tissue consumed per predator per $\mathrm{m}^{2}$ per day) were then calculated based on the multispecies Type II functional response (on which Eqn. 5.6 is based)

$$
C_{i}=\frac{m_{i} c_{i} N_{i}}{1+\sum_{k=1}^{S} c_{k} h_{k} N_{k}}
$$

[125] where $S$ is the total number of prey observed in the predator's diet and $m_{i}$ is the 
$i^{\text {th }}$ prey's average dry tissue weight. We calculated prey weight from the sizes of prey items observed during the feeding surveys using species-specific allometric relationships [129]. Feeding rates were converted to proportional diet contributions for comparison to those inferred using stable isotope data (Table 5.1).

\subsubsection{Stable isotope estimates of prey contributions}

Individuals of $H$. scobina (12-15 mm shell length, $n=10)$ and each of its observed prey ( $n=5-8$ per species) were collected for stable isotope analysis from both high and mid-intertidal zones in July of 2004 and 2005. All individuals were stored live on ice for $4 \mathrm{hrs}$ and frozen prior to processing. Insufficient material was obtained for $N r$ to enable analysis. Although only a single $N r$ predation event was observed during feeding surveys, we account for this missing prey source by substituting $N r$ with the limpet Patelloida corticata $(P c)$, a closely related species that shares the same habitat (Table 5.2). Analysis of the system without $P c$ did not change the applicability of our weighting method (Fig. S5.2).

We dissected, rinsed, and oven-dried the foot muscle tissue of all snails and limpets $(H s, A a, A c, P c, R v)$ and the whole-body tissue of the mussels $(M g, X p)$ and barnacles $(C c, E p)$. The ground samples of some species were pooled to ensure sufficient sample size (Table 5.2). Analyses of carbon and nitrogen stable isotope ratios were performed by the UC Davis Stable Isotopes Facility and were expressed in delta-notation such that $\delta=1000\left(\left(R_{\text {sample }} / R_{\text {standard }}\right)-1\right)$ where $R=$ either ${ }^{13} \mathrm{C} /{ }^{12} \mathrm{C}$ or ${ }^{15} \mathrm{~N} /{ }^{14} \mathrm{~N}$;

reference standards are Vienna PeeDee-belemnite for carbon and atmospheric $\mathrm{N}_{2}$ for 
nitrogen. The isotope values of the predator $H s$ were corrected by $-1 \pm 0.2 \%$ and $-2 \pm 0.3 \%$ for $\delta^{13} \mathrm{C}$ and $\delta^{15} \mathrm{~N}$, respectively, to account for trophic enrichment ( $\left.\pm \mathrm{SD}\right)$. Chosen values are representative of those observed for invertebrates in general [194].

\subsection{Results}

\subsubsection{Hypothetical dataset and model assessment}

A hypothetical predator-prey isotopic dataset (Fig. 5.1A) was assigned a single consumer species, and four prey species, each normally distributed across the bivariate isotopic niche space (consumer and prey standard deviation $(\mathrm{SD})=0.5$ for each isotopic tracer). Two prey were constructed to have distinct (non-overlapping) isotopic distributions, while the blue and orange prey (hereafter referred to as prey $i$ and $j$, respectively) were given equal values. The mixing space was designed such that the overlap of prey $i$ and $j$ could be manipulated without affecting direct mixing model output (Fig. S5.3). As expected, analysis of this system with a Bayesian isotope mixing model (MixSIR v.1.0.4) provided similar posterior probability distributions of contribution-to-diet values for the two overlapping prey species (Fig. 5.1B): proportional contribution medians ( $1^{\text {st }}$ quartile, $3^{\text {rd }}$ quartile) for prey $i$ and $j=0.17(0.08,0.25), 0.16(0.08,0.25)$. To evaluate isotopic similarity between prey $i$ and $j$, we employed Pianka's measure of density overlap, such that $w_{i j}=1$. We then set prey availability values $\left(\alpha_{i}, \alpha_{j}\right)$ to 0.1 and 0.9 for prey $i$ and $j$, respectively, and applied these values to Eqs. 5.4 and 5.5 across the entire matrix of iterated diet-to-contribution vectors to calculate the revised estimates 
of dietary reliance (Fig. 5.1C): revised proportional contribution medians (1st quartile, 3rd quartile) for prey $i$ and $j=0.04(0.01,0.09), 0.30(0.25,0.32)$.

An examination of Pianka's measure of overlap confirms consistent behavior over a range of isotopic mean differences and variances, resulting in a sigmoid relationship between the mean difference of prey isotope values and $w_{i j}$ that becomes less pronounced with increased variance (Fig. 5.2A). Because we constructed the mixing space such that estimates of dietary contribution are nearly invariant with respect to manipulation of $w_{i j}$ (cf. Fig. S5.3), an assessment of the behavior of our weighting procedure across different ratios of prey availability is possible (Fig. 5.2B,C). There is a linear relationship between the initial degree of isotopic overlap of two prey and the difference of final posterior probability distribution mean values. The slope of this relationship is determined by the availability differences $\left(\alpha_{i}-\alpha_{j}\right)$; small variations in the linear trends can be attributed to fluctuations in mean values as the distributions are successively weighted and renormalized. If the abundances of prey $i$ and $j$ are equal $\left(\alpha_{i} \alpha_{j}=0\right)$, our weighting procedure returns results identical to those originally calculated by the isotope mixing model, as intended given its a priori ultrageneralist assumption. As the overlap of prey isotope values increases, the weighting procedure increasingly returns dietary contribution values influenced by the relative availability of the two isotopically similar prey. This translates to an increasing discrepancy between the proportional contribution to diet distributions of prey $i$ and $j$ as the difference in availability increases. 


\subsubsection{New Zealand dataset and model validation}

Haustrum scobina's diet at the site includes eight potential prey ( $A a, A c$, $C c, E p, M g, P c$ (substituted for $N r$ ), $R v$, and $X p$ ). Five of the eight prey species are isotopically distinct, however the nesting mussel Xenostrobus pulex $(X p)$ and the blue mussel Mytilus galloprovincialis $(M g)$ had overlapping isotope values; $w_{i j}=0.63$ (Fig. 5.3A). An analysis of the mixing space reveals a range of likely contributions for each prey (Fig. 5.3B). The isotopic similarity between $X p$ and $M g$ resulted in similar contribution-to-diet probability distributions for these species in unweighted MixSIR results (Fig. 5.3B; blue and green hatched distribution density lines, respectively): $M g=0.16(0.07,0.28), X p=0.18(0.08,0.31)$; median (1st quartile, 3rd quartile). Applying relative abundance measurements $\left(\alpha_{M g}=0.05, \alpha_{X p}=0.95\right)$ to our weighting procedure resulted in proportional contribution distributions that were quite different (Fig. 5.3C): $M g=0.07(0.03,0.16), X p=0.27(0.15,0.41)$.

To investigate whether our weighting procedure improved inferences of prey contribution to the diet of $H s$, we regressed both original mixing model results and our weighted results against Haustrum scobina's field-estimated proportional feeding rates on each of its prey species (Fig. 5.3D,E). Recall that our field-estimated feeding rates were weighted by prey biomass to ensure a more direct comparison to the isotopic representation of the system, which is intrinsically mass-dependent (Eq. 5.7). The ln-lntransformed regression of the mixing model result means with feeding rate observation means (Fig. 5.3D) indicated that four of the five most heavily preyed upon species 
(prey with predator feeding rates greater than 0.0023 grams $\cdot$ whelk $^{-1} \cdot$ day $^{-1}$ ), with the exception of $M g$, fall within the $90 \%$ confidence interval (slope $=0.19$, and $\mathrm{R}^{2}=$ 0.46). After the implementation of our weighting procedure, all five of the top prey species fall within the $90 \%$ confidence interval (Fig. 5.3E; slope $=0.22, \mathrm{R}^{2}=0.56$ ). Accordingly, the inclusion of prey availability, conditional on the isotopic uncertainty of prey, increased the slope and improved the accuracy of trophic interaction predictions by ca. $10 \%$.

\subsection{Discussion}

The usefulness of a model is ranked by its ability to accurately describe reality with a minimal number of assumptions [70]. A Bayesian isotope mixing model with an uninformative prior assumes that the consumer is an ultrageneralist until proven otherwise. When multiple prey have similar isotope values, the ultrageneralist assumption becomes realized in the mixing model results, forcing the consumer to have equal contributions of each prey source. Because ultrageneralism is an unrealistic assumption in most cases, we developed a procedure by which different types of biological information can be used to revise this assumption. Our method enables partitioning of prey that are isotopically similar but differ in their relative availability to a predator. We reiterate that prey availability values could represent proportional differences in available biomass, predator preference, relative digestibility, or any (combination of) measurements influencing the availability of prey biomass to a predator. As such, the inclusion 
of neutral interaction assumptions (consumption in proportion to the prey's abundance) is conditioned on the isotopic uncertainty of prey; the isotope values of prey that are isotopically distinct remain dominantly informative.

Our isotopic analysis of a New Zealand intertidal system, compared to fieldmeasured feeding rates, reveals increased accuracy in predicted consumption rates of Haustrum scobina on its prey after the weighting procedure was employed (Fig. 5.3D,E). Neutral interaction assumptions were incorporated only for isotopically similar prey (the mussels Xenostrobus pilex and Mytilus galloprovincialis), thereby avoiding the incorporation of such assumptions for all prey. Our results suggest that, in this case, we interchange the formalism of the Bayesian paradigm with predictive power, thereby validating the use of such methods in cases where neutral interaction assumptions cannot be justified, or when appropriate prior probability distributions cannot be constructed, for all potential prey.

The ultimate aim of our method is to increase the accuracy of trophic interaction estimates, thereby contributing to our understanding of ecological systems, the role of neutral and niche processes in organizing communities, and the structure of food webs. Here we address the issue of isotopic similarity concerning a pair of prey, however in more complex scenarios, multiple iterations of this method could be carried out for multiple pairs of overlapping prey. In principle, it would also be possible to handle larger numbers of overlapping prey within the framework that we have presented, and this would be a useful future addition to this work. Whether there are few or many isotopically overlapping resources, the ability of our method to refine estimates of prey 
contribution to a consumer's diet relies on the accurate incorporation of proportional prey availability data. Although the availability of a prey may be the result of differential abundance (emphasized here), it may also result from consumer behavior, where certain prey are preferred over others. As long as prey availability can be expressed as proportions (where the proportional availability sums to unity over the isotopically overlapping prey), such data can be used in a manner equivalent to our example of differential prey abundance. The accuracy of our method is entirely dependent on the accuracy of proportional availability measurements. If prey availability data are highly uncertain or unknown for one or more of the isotopically overlapping prey sources, our method is of little utility. Furthermore, if multiple mechanisms are important in constraining prey availability, the relative influence of each mechanism must be known such that the overall' proportional availability of a given prey can be estimated.

There are situations when it is difficult or impossible to inform Bayesian isotope mixing models with availability data. Such issues contribute to difficulties in developing suitable priors for prey species in many systems, though an exploration of prior development in the context of consumer-resource relationships, particularly for isotope mixing models, deserves additional investigation. Formulation of prior distributions based on availability data may be possible and appropriate in systems that are observable and where neutral interaction assumptions can be justified. However, ratios of stable isotopes are often considered to be of greatest utility when used to investigate systems that are difficult, if not impossible, to observe directly [85, 128, 84]. Our procedure enables investigators to employ independent biological information to gain additional insights 
from isotopic data, while simultaneously relaxing the number of required parameters relative to traditional Bayesian models of consumer resource use.

\section{Acknowledgements}

We thank C.E. Chow, C.T. Darimont, K. Fox-Dobbs, J. Hopkins, S. Kim, H. Lee, M. Mangel, S.D. Newsome, P.V. Wheatley, and two anonymous reviewers for helpful comments and discussions that greatly improved the quality of this manuscript. 
5.5 Tables 


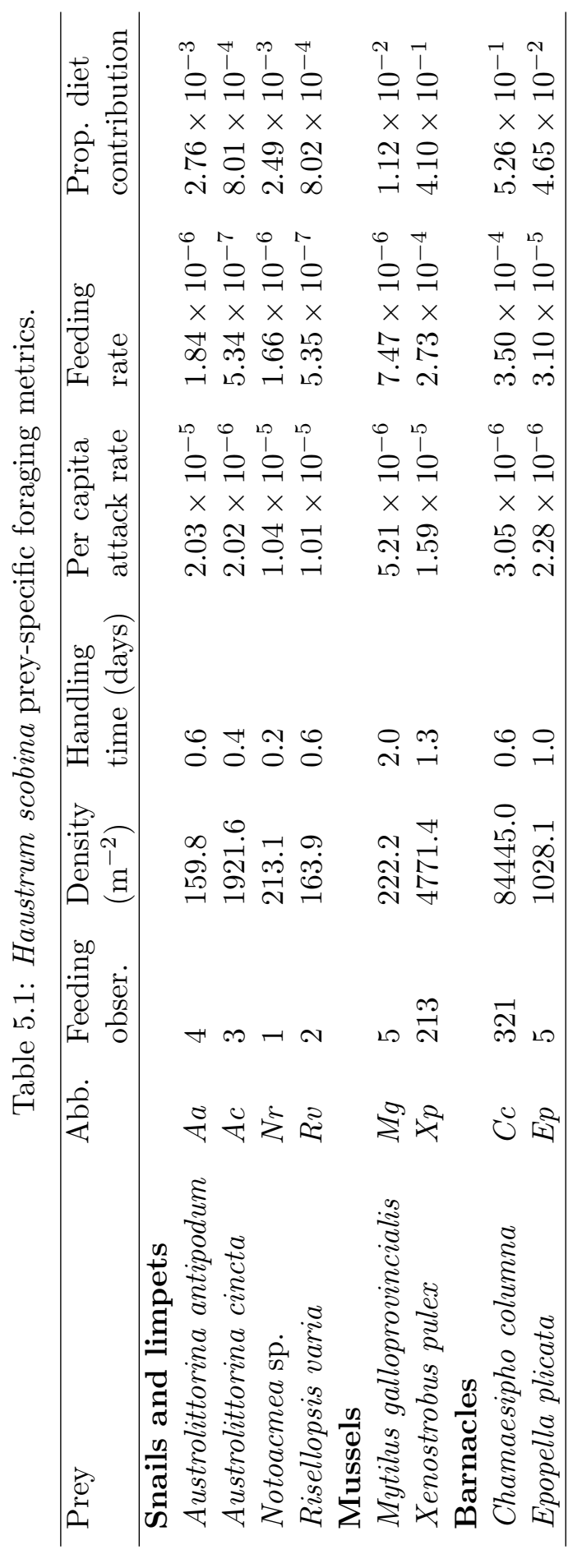


Table 5.2: Mean and standard deviation of species-specific isotopic signatures at Tauranga Head, New Zealand.

\begin{tabular}{|c|c|c|c|c|c|c|}
\hline \multirow[t]{2}{*}{ Species } & \multirow[t]{2}{*}{ Abb. } & \multicolumn{2}{|l|}{$\delta^{15} \mathrm{~N}(\%)$} & \multicolumn{2}{|c|}{$\delta^{13} \mathrm{C}(\%)$} & \multirow[t]{2}{*}{$\mathrm{n}$} \\
\hline & & Mean & St. Dev. & Mean & St. Dev. & \\
\hline \multicolumn{7}{|l|}{ Whelk Predator } \\
\hline Haustrom scobina & $H s$ & 10.4 & 0.7 & -17.1 & 0.3 & 7 \\
\hline \multicolumn{7}{|l|}{ Snails and limpets } \\
\hline Austrolittorina antipodum & $A a$ & 7.6 & 0.1 & -14.8 & 0.3 & 5 \\
\hline Austrolittorina cincta & $A c$ & 8.3 & 0 & -17.8 & 0.7 & 2 \\
\hline Patelloida corticata & $P c$ & 9.3 & 0.4 & -11.9 & 0.2 & 5 \\
\hline Risellopsis varia & $R v$ & 8.7 & 0.1 & -15.3 & 0.4 & 5 \\
\hline \multicolumn{7}{|l|}{ Mussels } \\
\hline Mytilus galloprovincialis & $M g$ & 7.7 & 0.2 & -18.6 & 0.3 & 5 \\
\hline Xenostrobus pulex & $X p$ & 7.9 & 0.4 & -18.8 & 0.2 & 5 \\
\hline \multicolumn{7}{|l|}{ Barnacles } \\
\hline Chamaesipho columna & $C c$ & 9.3 & 0.3 & -18.6 & 0.6 & 7 \\
\hline Epopella plicata & $E p$ & 11.6 & 0.4 & -18.7 & 0.2 & 7 \\
\hline
\end{tabular}




\subsection{Figures}

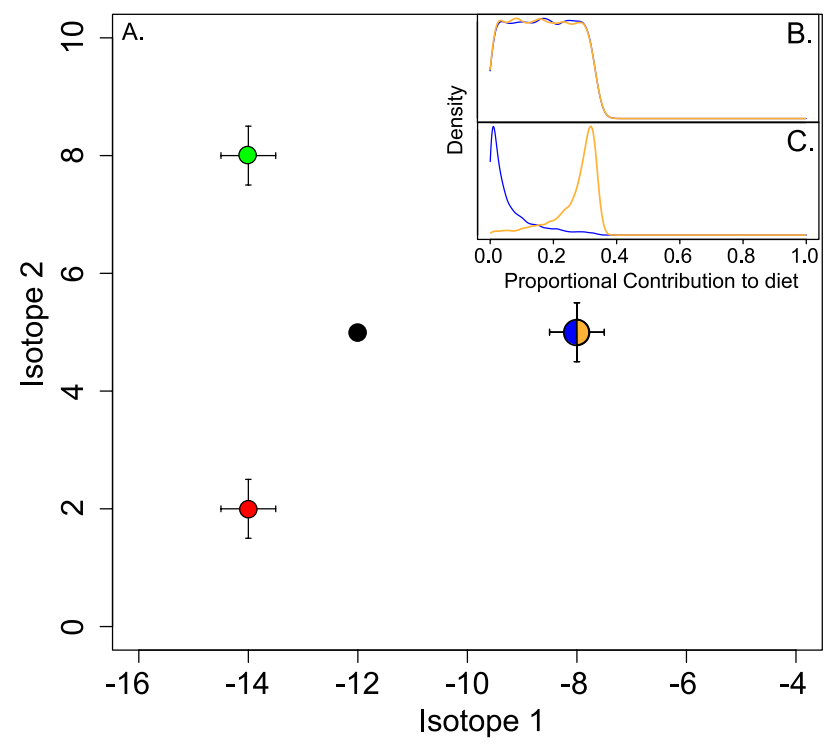

Figure 5.1: Isotopic niche space of a theoretical single predator, four prey community. A. The predator (black) and prey (red, green, blue, orange) have a standard deviation of $(0.5,0.5)$ for both isotope tracer 1 and 2 . Two of the four prey (red and green) are isotopically distinct with no overlap. Two of the four prey (blue and orange) are isotopically identical, with $w_{i j}=1$. B. Initial MixSIR contribution-to-diet posterior probability distributions for the isotopically identical prey. C. Final weighted and renormalized posterior probability distributions for the isotopically similar prey. Relative abundance values of 0.9 and 0.1 were applied to the orange and blue prey, respectively. doi:10.1371/journal.pone.0022015.g001 

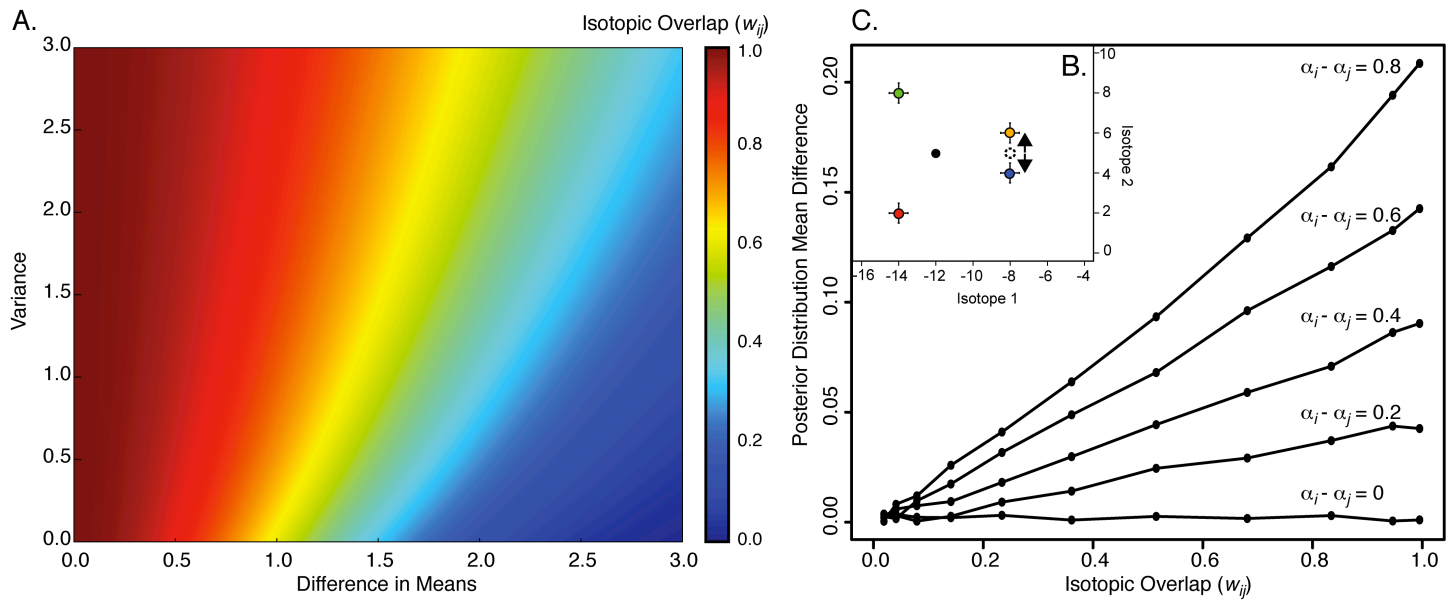

Figure 5.2: Sensitivity analysis of the weighting procedure as a function of isotopic similarity and the difference in proportional availability of isotopically similar prey. A. Sensitivity analysis of Pianka's measure of density overlap $\left(w_{i j}\right)$ across differences in mean values and variance of two bivariate normal isotopic distributions. When variance is small, $w_{i j}$ decreases sigmoidally as the difference in means increases. Larger variance predictably tends to linearize the relationship. B. The mixing space of our hypothetical scenario permits manipulation of isotopic overlap between two prey (blue and orange; the consumer is black) without altering mixing model estimates of summed proportional contribution to diet of the two prey (cf. Fig. S5.3). C. An analysis of the effect of model parameters (density overlap, $w_{i j}$, and availability differences, $\alpha_{i}-\alpha_{j}$ ) on the final weighted and renormalized posterior distributions, measured by the difference of final distribution means for blue and orange in the hypothetical isotopic dataset (Fig. 5.1). The model is assessed across all possible values of $w_{i j}(0: 1)$, and across five prey availability scenarios. There is a strong linear effect of isotopic overlap on the difference in means of the weighted posterior probability distributions. The slope of this relationship is determined by availability differences. doi:10.1371/journal.pone.0022015.g001 

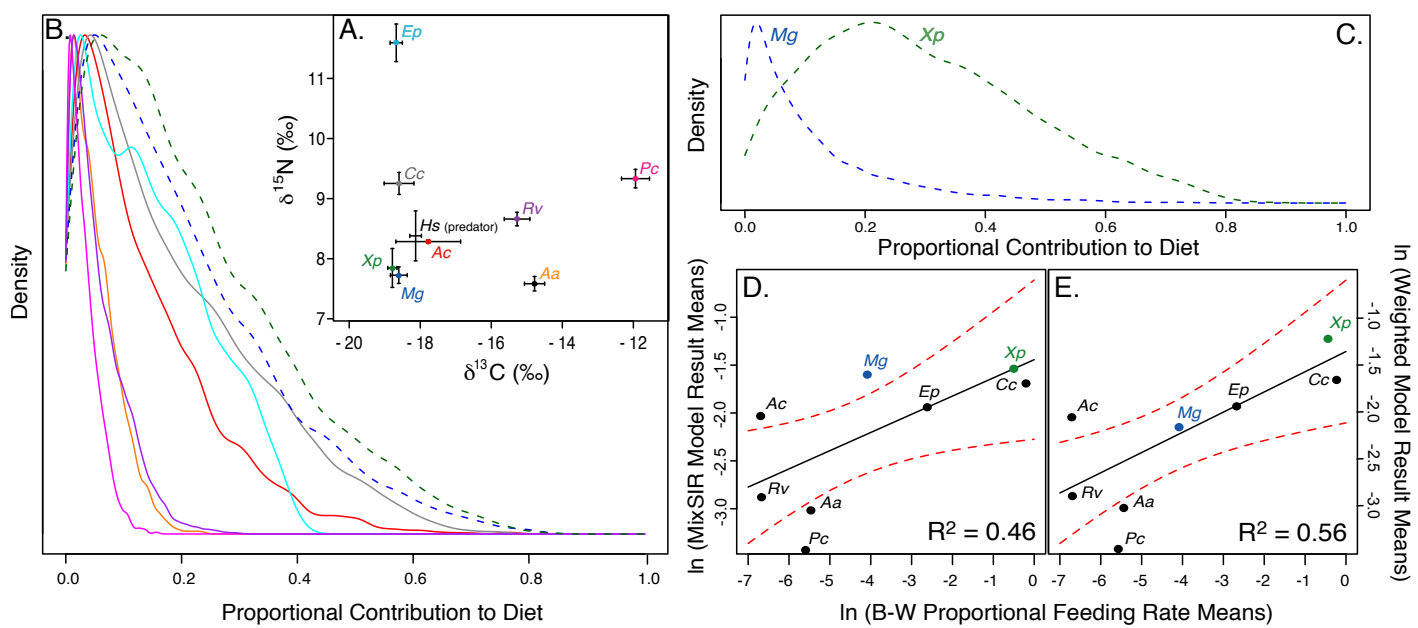

Figure 5.3: The weighting procedure applied to a New Zealand intertidal community. A. Isotopic niche space of the New Zealand whelk- predator system, where $\delta^{13} \mathrm{C}$ is plotted on the $\mathrm{x}$-axis and $\delta^{15} \mathrm{~N}$ is plotted on the y-axis. The $\delta^{13} \mathrm{C}$ and $\delta^{15} \mathrm{~N}$ values of the predator Hs are adjusted for trophic discrimination factors (see methods). B. MixSIR contribution-to-diet posterior probability distributions of all New Zealand prey for predator Hs. Solid distribution density lines denote isotopically distinct prey, whereas hatched distribution density lines denote isotopically similar prey. Prey colors match those of panel 3A. C. Weighted posterior probably distributions for the isotopically similar prey. Relative abundance values of 0.05 and 0.95 correspond to $M g$ (blue) and $X p$ (green), respectively. D. ln-ln-transformed regression of biomassweighted proportional feeding rate means vs. original mixing model result means; (slope $=0.19$, and $\mathrm{R}^{2}=0.46$ ). Hatched red lines represent the $90 \%$ confidence interval. $\mathbf{E}$. ln-ln-transformed regression of biomass-weighted proportional feeding rate means vs. weighted model result means; slope $=0.22, \mathrm{R}^{2}=0.56$. Hatched red lines represent the 90\% confidence interval. doi:10.1371/journal.pone.0022015.g003 


\subsection{Supplementary Material}

\subsubsection{Figures}

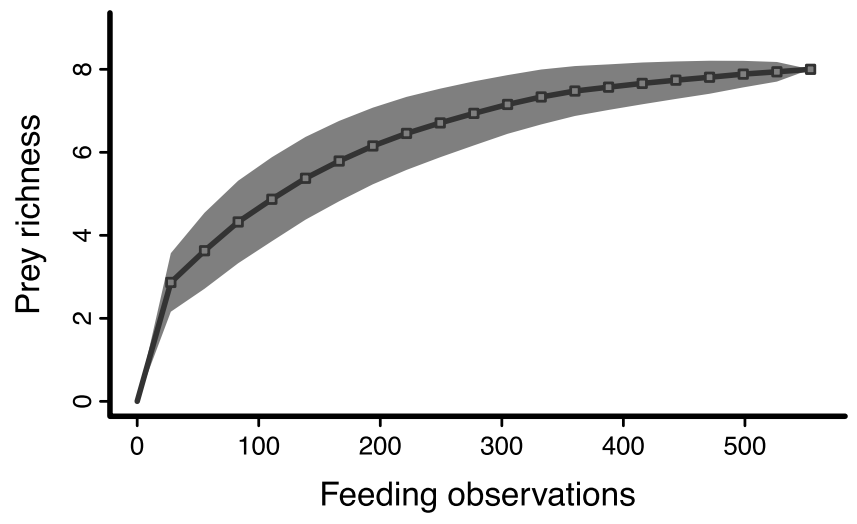

Supplementary Figure 5.1: Species accumulation curve ( \pm SD) of the prey observed in the diet of Haustrum scobina at Tauranga Head, constructed using feeding survey observations as the unit of sampling 
A.

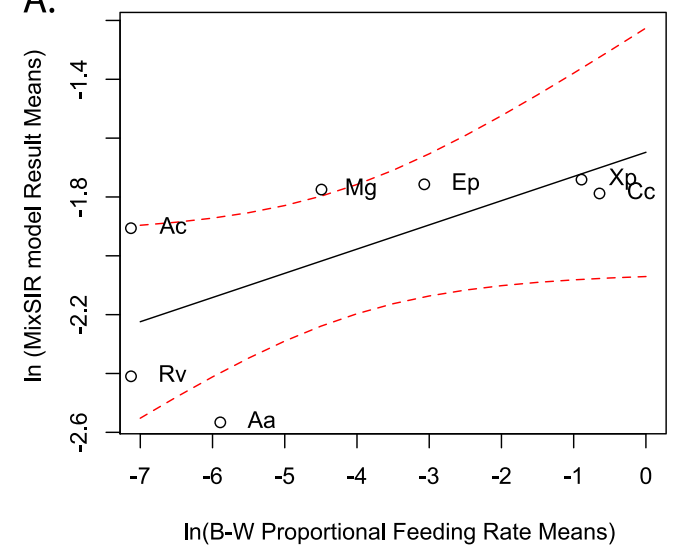

B.

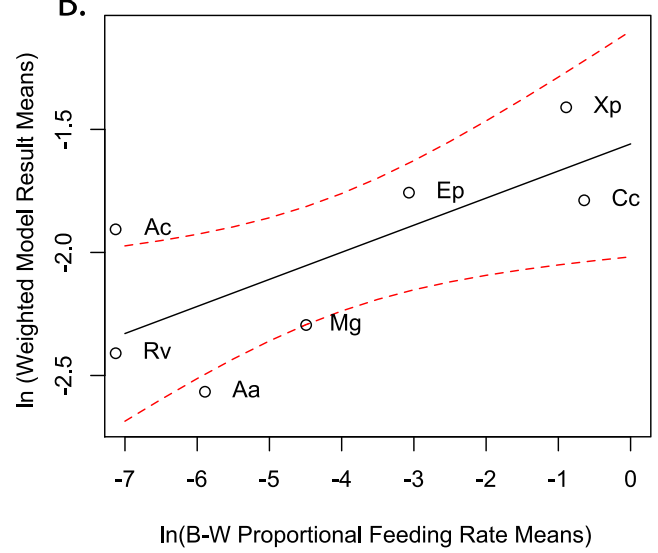

Supplementary Figure 5.2: An analysis of the New Zealand system excluding the prey Patelloida corticata $(P c)$. As expected, there are slight differences in original MixSIR results, however, applying prey availability data to the overlapping prey $M g$ and $X p$ results in a similar increase in the accuracy of prey contribution estimations. A. ln-lntransformed regression of biomass-weighted proportional feeding rate means vs. original mixing model result means; slope $=0.08, \mathrm{R}^{2}=0.42$. B. ln-ln-transformed regression of biomass-weighted proportional feeding rate means vs. weighted model result means; slope $=0.11, \mathrm{R}^{2}=0.53$. The hatched red lines represent the $90 \%$ confidence interval. 

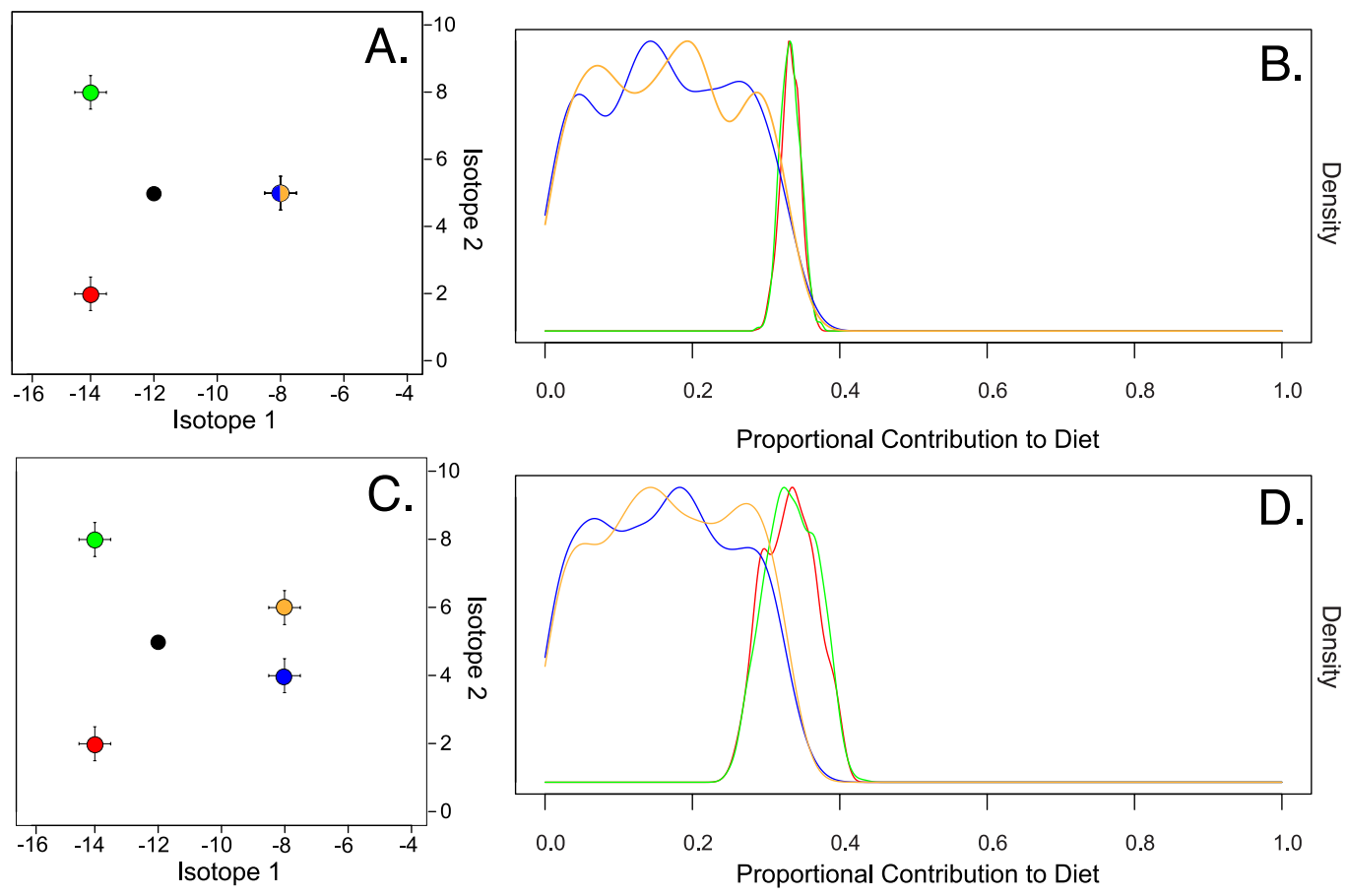

Supplementary Figure 5.3: Manipulation of the isotopic overlap of prey. A. A mixing space with 2 non-overlapping prey (green, red), two overlapping prey (blue, orange), and a single consumer (black). Here, the overlap of blue and orange prey $\left(w_{\text {blue, orange }}=1\right)$. B) MixSIR estimates of $\%$ contribution to diet for each prey associated with Fig. S5.3A. Green and red are predicted to contribute equally to the consumer's diet, as are blue and orange. C. An alternative mixing space such $w_{\text {blue, orange }}=0$. Here, the blue and orange prey have been symmetrically moved from their previous isotopic values $\left(\delta^{13} \mathrm{C}\right.$ $\left.=28, \delta^{15} \mathrm{~N}=5\right)$ to Blue: $\delta^{13} \mathrm{C}=28, \delta^{15} \mathrm{~N}=4$; Orange: $\delta^{13} \mathrm{C}=28, \delta^{15} \mathrm{~N}=6$. D. MixSIR estimates of $\%$ contribution to diet for each prey associated with Fig. S5.3C. Although the overlap of blue and orange has been manipulated, the geometry of the mixing space retains similar estimates of $\%$ contribution to diet for all prey, with only slight differences in variance. 


\section{Chapter 6}

\section{Synthesis}

We remember the past, we do not remember the future. We have a different kind of awareness about what might happen than we have of what probably has happened.

-Richard Feynman (The Character of Physical Law, 1964)

Feynman captures two important elements addressed in this thesis. First, the past and the future are linked, which is well understood superficially, but less well understood specifically. Second, our understanding of the past is inherently probabilistic. My goal here is to quantify the relationship between link-strength variance and foodweb structure, its measurability in systems that cannot be experimentally manipulated, and to both predict and inform smaller-scale patterns of interaction between consumers and their resources. These methods are not limited to modern systems, and are used to elucidate both large- and small-scale patterns of interaction in mammalian communities with temporal spans ranging from the recent to the Plio-Pleistocene. Reconstructing species interactions in past ecosystems has a rich history [85], and recent investigations 
have demonstrated that our understanding of the past can facilitate knowledge of modern ecosystems $[39,86,126]$. Given the scale over which interactions occur, particularly for systems with long-lived organisms, historical and paleontological approaches offer windows into nature unavailable to modern ecology. These windows enable observations (however indirect) of interactions over large time-scales and across environmental and climatic perturbations, many of which have characteristics analogous to predicted future climate scenarios (cf. [30, 31]).

An integration of modern ecological approaches with those used to study past ecosystems serves to mitigate their individual limitations. The study of modern consumer-resource interactions is temporally limited, while studies of past interactions must address issues including the temporal resolution of communities [59] and biases in the fossil record [8]. Although these issues may serve to limit empirical approaches, process-based modeling is well-suited to formulate an investigator's assumptions regarding consumer-resource interactions, and is useful for both predicting and informing independent lines of evidence $[109,41]$. Such approaches are more common in modern ecological applications, and are well-suited for, but underutilized in, paleo-ecological studies.

The results from Chapter 1 demonstrate that link-strength variance can be hierarchically described, variance at the level of the food-web (Network Level Interaction Distribution, NLID) is predictive of the general structural organization of species interactions, and that this distribution can be estimated when data are limited. This is important, because it suggests that structure is - in part - independent of the ecolog- 
ical tradeoffs that constrain interactions between consumers and resources. Moreover, the distribution of specialists and generalists in the system appear to be predictive of NLID shape. Recent investigations of non-antagonistic systems (such as mutualistic networks) show that the distribution of specialists and generalists has large implications on network structure [65, 64], and that these structures often result from coevolutionary relationships. By comparison, an understanding of evolutionary forces that shape antagonistic networks is not well-developed.

In Chapter 2, I applied the methods developed in Chapter 1 to examine six paleontological food-webs across Eurasia and spanning the Last Glacial Maximum (LGM). This work represents the first empirical analysis of quantitative food-web structure for paleontological systems. The scale at which interactions are assessed provides insight regarding how food-web structures change over large spatial scales, over long periods of time, and whether they were impacted by the LGM, a global climatic event. The results demonstrate that food-webs with strong species contiguity can differ in structure, and that these structures did appear to be influenced by the LGM, particularly in Beringia. Although this investigation is primarily observational, the results are suggestive of potential mechanisms that drove the structural differences observed in Europe and Beringia. Interactions examined at a species-specific scale shared strong differences in patterns of prey utilization among predators across Eurasia. Felids appeared to have more constrained dietary proclivities, while the diets of Canis and Ursus were more idiosyncratic. Moreover, our results can be assessed with respect to reconstructions of herbivore abundances across the LGM [99], and we show that predator diets (particu- 
larly felids) were strongly influenced by the increased abundance and range expansion of Rangifer in the post-Glacial.

Chapters 1 and 2 detail observational approaches towards understanding foodweb structure with respect to the constraints limiting species interactions, and Chapter 3 examines particular dietary constraints in a predictive framework. Here I present a process-based model to quantify the extent that foraging decisions are impacted by the mechanical wear of molar enamel among anthropoid, and specifically hominin, foragers. Model results predict that foods such as plant Underground Storage Organs (USOs) are consumed as fallback foods, as has been observed among modern hunter-gatherers [111]. However, I found that the importance of these resources (as determined by their contribution to decision matrices) appear to decrease as the organism's body size increases. Moreover, our model framework is used to determine fitness benefits of physically altering the mechanical properties of foods (i.e. mechanical advantages), compared against morphological adaptions thought to increase an organism's ability to consume fracture resistant resources. Our results demonstrate that mechanical advantages do provide fitness benefits, but only if the organism does not have morphological adaptations such as hyper-thickly enameled molars. Finally, we show how such predictive model frameworks can be used to build Bayesian priors, thus serving to inform independent paleo-dietary information.

Chapter 4 concerns the integration of prey availability information with Bayesian isotope mixing models to increase the accuracy of contribution-to-diet estimates. There have been many recent contributions concerning isotope mixing models - some introduce 
means by which uncertainty is integrated into dietary estimates [122, 139, 29, 73], while others concern geometrical analysis of the mixing space $[94,128,127]$. We integrate the two approaches, and use the results from mixing models, independent measures of prey availability, and mixing space geometry to improve dietary estimates.

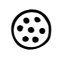

These studies span multiple scales of species interation (Fig. 1.2), but all consider field-based, rather than experimental, measures of interaction. This is disconcerting to some, because the definition of an interaction is constrained to that involving the flow of biomass between species. Moreover, the subsequent analysis of structure - particularly for paleontological systems - is chiefly observational rather than experimentally derived, limiting the understanding of potential processes driving observed patterns. Hilborn and Mangel state in 'The Ecological Detective': "Experimental falsifiability is not a rule, it is a tool, like mathematical modeling, statistical inference, a pair of binoculars, or an electron microscope." [70]. In this thesis, ratios of stable isotopes serve as binoculars, and we present approaches whereupon such tools are combined with mathematical modeling to both observe and then predict consumer-resource interactions. Using a wide range of methodologies as well as integrating paleo-ecological information will contribute to a greater understanding of the myriad patterns of interactions in nature, and the forces from which they are shaped.

-Justin D Yeakel, May 23, 2012 


\section{Bibliography}

[1] P. Abrams and L. Ginzburg, The nature of predation: prey dependent, ratio dependent or neither?, Trends in Ecology and Evolution, 15 (2000), pp. 337-341.

[2] J. Agustí And M. Antón, Mammoths, sabertooths, and hominids, 65 million years of mammalian evolution in Europe, Columbia Univ Pr, Mar. 2002.

[3] R. Albert And A. Barabasi, Statistical mechanics of complex networks, Reviews of Modern Physics, 74 (2002), pp. 47-97.

[4] S. Allesina, D. Alonso, and M. Pascual, A General Model for Food Web Structure, Science, 320 (2008), pp. 658-661.

[5] S. Allesina And M. Pascual, Network structure, predator-prey modules, and stability in large food webs, Theor. Ecol., 1 (2008), pp. 55-64.

[6] S. Allesina And S. Tang, Stability criteria for complex ecosystems, Nature, (2012), pp. 1-4. 
[7] M. Almeida-Neto, P. Guimarães, P. R. Guimarães Jr, R. D. Loyola, AND W. UlRICH, A consistent metric for nestedness analysis in ecological systems: reconciling concept and measurement., Oikos, 117 (2008), pp. 1227-1239.

[8] J. Alroy, M. Aberhan, D. J. Bottjer, M. Foote, F. T. Fursich, P. J. Harries, A. J. W. Hendy, S. M. Holland, L. C. Ivany, W. Kiessling, M. A. Kosnik, C. R. Marshall, A. J. Mcgowan, A. I. Miller, T. D. Olszewski, M. E. Patzkowsky, S. E. Peters, L. Villier, P. J. Wagner, N. Bonuso, P. S. Borkow, B. Brenneis, M. E. Clapham, L. M. Fall, C. A. Ferguson, V. L. Hanson, A. Z. Krug, K. M. Layou, E. H. Leckey, S. Nurnberg, C. M. Powers, J. A. Sessa, C. Simpson, A. Tomasovych, And C. C. Visaggi, Phanerozoic Trends in the Global Diversity of Marine Invertebrates, Science, 321 (2008), pp. 97-100.

[9] S. H. Ambrose And M. J. DeNiro, The isotopic ecology of East African mammals, Oecologia, 69 (1986), pp. 395-406.

[10] M. S. Araújo, P. R. Guimarães Jr, R. Svanback, A. Pinheiro, P. Guimarẽes, S. F. Reis, And D. I. Bolnick, Network analysis reveals contrasting effects of intraspecific competition on individual vs. population diets, Ecology, 89 (2008), pp. 1981-1993.

[11] R. Barnett, B. Shapiro, I. Barnes, S. Y. W. Ho, J. Burger, N. Yamaguchi, T. F. G. Higham, H. T. Wheeler, W. Rosendahl, A. V. Sher, M. Sotnikova, T. Kuznetsova, G. F. Baryshnikov, L. D. Martin, C. R. 
Harington, J. A. Burns, And A. Cooper, Phylogeography of lions (Panthera leo ssp.) reveals three distinct taxa and a late Pleistocene reduction in genetic diversity, Molecular Ecology, 18 (2009), pp. 1668-1677.

[12] A. Barrat, M. Barthélemy, R. Pastor-Satorras, and A. Vespignani, The architecture of complex weighted networks, Proc. Natl. Acad. Sci. USA, 101 (2004), p. 3747.

[13] J. Bascompte, P. Jordano, C. J. Melián, and J. M. Olesen, The nested assembly of plant-animal mutualistic networks., Proc. Natl. Acad. Sci. USA, 100 (2003), pp. 9383-9387.

[14] J. Bascompte, C. J. Melián, And E. Sala, Interaction strength combinations and the overfishing of a marine food web, Proc. Natl. Acad. Sci. USA, 102 (2005), pp. $5443-5447$.

[15] E. B. Baskerville, A. P. Dobson, T. Bedford, S. Allesina, T. M. AnDerson, And M. Pascual, Spatial Guilds in the Serengeti Food Web Revealed by a Bayesian Group Model, PLoS Comp. Biol., 7 (2010), p. e1002321.

[16] G. Belovsky, An optimal foraging-based model of hunter-gatherer population dynamics, Journal of Anthropological Archaeology, 7 (1988), pp. 329-372.

[17] E. L. Berlow, J. A. Dunne, N. D. Martinez, P. B. Stark, R. J. Williams, AND U. Brose, Simple prediction of interaction strengths in complex food webs, Proc. Natl. Acad. Sci. USA, 106 (2009), p. 187. 
[18] E. L. Berlow, A. Neutel, J. E. Cohen, P. C. De Ruiter, B. Ebenman, M. Emmerson, J. W. Fox, V. A. A. Jansen, J. I. Jones, G. D. Kokkoris, D. O. Logofet, A. J. McKane, J. M. Montoya, and O. Petchey, Interaction strengths in food webs: issues and opportunities, J. Anim. Ecol., 73 (2004), pp. $585-598$.

[19] N. Blüthgen, F. Menzel, And N. Blüthgen, Measuring specialization in species interaction networks., BMC Ecol., 6 (2006), p. 9.

[20] N. Boccara, Modeling complex systems, Springer, New York, Jan. 2004.

[21] H. Bocherens, Isotopic biogeochemistry and the paleoecology of the mammoth steppe fauna, in Advances in Mammoth Research, W. F. Reumer, F. Braber, D. Mol, and J. de Vos, eds., Deinsea, Rotterdam, 2003, pp. 57-76.

$[22]$ - Neanderthal dietary habits: Review of the isotopic evidence., in The evolution of hominin diets, Springer Verlag, June 2009.

[23] H. Bocherens, D. Billiou, And A. Mariotti, Palaeoenvironmental and palaeodietary implications of isotopic biogeochemistry of last interglacial Neanderthal and mammal bones in Scladina Cave (Belgium), Journal of Archaeological Science, 26 (1999), pp. 599-607.

[24] H. Bocherens, D. Billiou, A. Mariotti, M. Toussaint, M. PatouMathis, D. Bonjean, And M. OtTe, New isotopic evidence for dietary habits of Neandertals from Belgium, Journal of Human Evolution, 40 (2001), pp. 497-505. 
[25] H. Bocherens And D. Drucker, Trophic level isotopic enrichment of carbon and nitrogen in bone collagen: case studies from recent and ancient terrestrial ecosystems, Int. J. Osteoarchaeol., 13 (2003), pp. 46-53.

[26] H. Bocherens, D. Drucker, And D. Billiou, Bears and humans in Chauvet Cave (Vallon-Pont-d'Arc, Ardèche, France): Insights from stable isotopes and radiocarbon dating of bone collagen, J Hum Evol, (2006).

[27] H. Bocherens, D. G. Drucker, D. Billiou, M. Patou-Mathis, And B. VAndermeersch, Isotopic evidence for diet and subsistence pattern of the Saint-Césaire I Neanderthal: review and use of a multi-source mixing model, Journal of Human Evolution, 49 (2005), pp. 71-87.

[28] H. Bocherens, D. G. Drucker, D. Bonjean, A. Bridault, N. J. Conard, C. Cupillard, M. Germonpré, M. Höneisen, S. C. Münzel, H. NapierAla, M. Patou-Mathis, E. Stephan, H.-P. Uerpmann, and R. Ziegler, Isotopic evidence for dietary ecology of cave lion (Panthera spelaea) in NorthWestern Europe: Prey choice, competition and implications for extinction, Quaternary International, (2011), pp. 1-13.

[29] A. L. Bond And A. W. Diamond, Recent Bayesian stable-isotope mixing models are highly sensitive to variation in discrimination factors, Ecol. Appl., 21 (2012), pp. 1017-1023.

[30] G. Bowen, W. Clyde, P. Koch, S. Ting, J. Alroy, T. Tsubamoto, 
Y. WAng, And Y. WAng, Mammalian dispersal at the Paleocene/Eocene boundary, Science, 295 (2002), pp. 2062-2065.

[31] G. J. Bowen, D. J. Beerling, P. L. Koch, J. C. Zachos, and T. QuattleBAum, A humid climate state during the Palaeocene/Eocene thermal maximum., Nature, 432 (2004), pp. 495-499.

[32] D. Boyer, G. Ramos-Fernandez, O. Miramontes, J. L. Mateos, G. CoCho, H. Larralde, H. Ramos, and F. Rojas, Scale-free foraging by primates emerges from their interaction with a complex environment, Proc. Roy. Soc. B, 273 (2006), pp. 1743-1750.

[33] C. K. Brain, The hunters or the hunted?, An introduction to African cave taphonomy, Univ of Chicago Pr, 1981.

[34] A. M. Bronikowski, J. Altmann, D. K. Brockman, M. Cords, L. M. Fedigan, A. Pusey, T. Stoinski, W. F. Morris, K. B. Strier, and S. C. Alberts, Aging in the Natural World: Comparative Data Reveal Similar Mortality Patterns Across Primates, Science, 331 (2011), pp. 1325-1328.

[35] L. B. Brubaker, P. M. Anderson, M. E. Edwards, and A. V. Lozhkin, Beringia as a glacial refugium for boreal trees and shrubs: new perspectives from mapped pollen data, J Biogeogr, 32 (2005), pp. 833-848.

[36] J. Bunbury And K. Gajewski, Postglacial climates inferred from a lake at 
treeline, southwest Yukon Territory, Canada, Quaternary Science Reviews, 28 (2009), pp. 354-369.

[37] D. Cajus G, Upper Pleistocene Panthera leo spelaea (Goldfuss, 1810) remains from the Bilstein Caves (Sauerland Karst) and contribution to the steppe lion taphonomy, palaeobiology and sexual dimorphism, Annales de paléontologie, 95 (2009), pp. 117-138.

[38] J. Camacho, R. Guimerà, and L. A. Nunes Amaral, Robust patterns in food web structure., Phys. Rev. Lett., 88 (2002), pp. 2281021-2281024.

[39] C. P. Chamberlain, J. R. Waldbauer, K. Fox-Dobbs, S. D. Newsome, P. L. Koch, D. R. Smith, M. E. Church, S. D. Chamberlain, K. J. SorenSOn, AND R. Risebrough, Pleistocene to recent dietary shifts in California condors, Proc. Natl. Acad. Sci. USA, 102 (2005), pp. 16707-16711.

[40] Y. Chen, C.-L. Sun, And M. Kanaiwa, Impacts of prior mis-specification on Bayesian fisheries stock assessment, Marine and Freshwater Research, 59 (2008), pp. $145-156$.

[41] C. W. Clark and M. Mangel, Dynamic state variable models in ecology: methods and applications, Oxford University Press, USA, 2000.

[42] P. U. Clark, A. S. Dyke, J. D. Shakun, A. E. Carlson, J. Clark, B. Wohlfarth, J. X. Mitrovica, S. W. Hostetler, and A. M. McCabe, The Last Glacial Maximum, Science, 325 (2009), pp. 710-714. 
[43] D. Codron, J. Codron, J. A. Lee-Thorp, M. Sponheimer, D. Ruiter, AND J. S. BRINK, Stable isotope characterization of mammalian predator-prey relationships in a South African savanna, Eur J Wildlife Res, 53 (2007), pp. 161170.

[44] J. E. Cohen, F. Briand, And C. M. Newman, Community food webs, data and theory, Springer-Verlag, 1990.

[45] P. J. Constantino, J. J. W. Lee, H. Chai, B. Zipfel, C. Ziscovici, B. R. LAWn, AND P. W. LuCAS, Tooth chipping can reveal the diet and bite forces of fossil hominins, Biology Letters, 6 (2010), pp. 826-829.

[46] S. Copeland, Paleoanthropological implications of vegetation and wild plant resources in modern savanna landscapes, with applications to Plio-Pleistocene Olduvai Gorge, Tanzania, PhD thesis, Ph.D. Dissertation, Rutgers University, 2004.

[47] E. L. Crow And K. Shimizu, Lognormal distributions, Theory and applications, Marcel Dekker, INC, New York and Basel, 1988.

[48] L. Dalén, L. Orlando, B. Shapiro, and M. Durling, Partial genetic turnover in neandertals: continuity in the east and population replacement in the west, Molecular Biology and ..., (2012).

[49] C. T. Darimont, P. C. Paquet, and T. E. Reimchen, Landscape heterogeneity and marine subsidy generate extensive intrapopulation niche diversity in a large terrestrial vertebrate, J. Anim. Ecol., 78 (2009), pp. 126-133. 
[50] T. P. Dawson, S. T. Jackson, J. I. House, I. C. Prentice, and G. M. MACE, Beyond predictions: biodiversity conservation in a changing climate., Science, 332 (2011), pp. 53-58.

[51] N. J. Dominy, E. R. Vogel, J. D. Yeakel, P. J. Constantino, and P. W. Lucas, Mechanical Properties of Plant Underground Storage Organs and Implications for Dietary Models of Early Hominins, Evol Biol, 35 (2008), pp. 159-175.

[52] D. G. Drucker, H. Bocherens, And D. Billiou, Evidence for shifting environmental conditions in Southwestern France from 33000 to 15000 years ago derived from carbon-13 and nitrogen-15 natural abundances in collagen of large herbivores, Earth and Planetary Science Letters, 216 (2003), pp. 163-173.

[53] R. I. M. Dunbar, Socioecology of the extinct theropiths: a modelling approach, in Theropithecus: the rise and fall of a primate genus, Cambridge Univ Pr, 2005.

[54] J. A. Dunne, The network structure of food webs, in Ecological networks: linking structure to dynamics in food webs, Oxford University Press, USA, 2006, pp. 2780.

[55] J. A. Dunne, R. J. Williams, and N. D. Martinez, Food-web structure and network theory: the role of connectance and size, Proc. Natl. Acad. Sci. USA, 99 (2002), pp. 12917-12922.

[56] T. Duong, ks: Kernel density estimation for bivariate data, 2009. 
[57] M. A. Fortuna, D. B. Stouffer, J. M. Olesen, P. Jordano, D. Mouillot, B. R. Krasnov, R. Poulin, and J. Bascompte, Nestedness versus modularity in ecological networks: two sides of the same coin?, J. Anim. Ecol., 79 (2010), pp. 811-817.

[58] K. Fox-Dobbs, J. K. Bump, R. O. Peterson, D. L. Fox, and P. L. Koch, Carnivore-specific stable isotope variables and variation in the foraging ecology of modern and ancient wolf populations: case studies from Isle Royale, Minnesota, and La Brea, Can. J. Zool., 85 (2007), pp. 458-471.

[59] K. Fox-Dobbs, J. LeOnard, And P. Koch, Pleistocene megafauna from eastern Beringia: Paleoecological and paleoenvironmental interpretations of stable carbon and nitrogen isotope and radiocarbon records, Palaeogeogr Palaeocl, 261 (2008), pp. $30-46$.

[60] B. FRY, Stable isotope ecology, Springer Verlag, New York, 2006.

[61] P. A. Garber, Foraging Strategies among Living Primates, Annual Review of Anthropology, 16 IS - (1987), pp. 339-364.

[62] A. Gelman, J. B. Carlin, H. S. Stern, and D. B. Rubin, Bayesian Data Analysis, Chapman \& Hall/CRC Press, New York, 2004.

[63] C. Griffith And B. Long, HOMINIDS: An agent-based spatial simulation model to evaluate behavioral patterns of early Pleistocene hominids, Ecological Modelling, 221 (2010), pp. 738-760. 
[64] P. R. Guimarães, P. Jordano, and J. N. Thompson, Evolution and coevolution in mutualistic networks., Ecol. Lett., 14 (2011), pp. 877-885.

[65] P. R. Guimarães Jr, V. Rico-Gray, P. S. Oliveira, T. J. Izzo, S. F. dos ReIs, And J. N. Thompson, Interaction intimacy affects structure and coevolutionary dynamics in mutualistic networks, Current Biology, 17 (2007), pp. 17971803.

[66] R. Guimerì And L. A. N. Amaral, Cartography of complex networks: modules and universal roles, J. Stat. Mech., 2005 (2005), p. P02001.

[67] R. Guimerà, D. B. Stouffer, M. Sales-Pardo, E. A. Leicht, M. E. J. Newman, And L. A. N. Amaral, Origin of compartmentalization in food webs., Ecology, 91 (2010), pp. 2941-2951.

[68] D. Guthrie, Origin and causes of the mammoth steppe: a story of cloud cover, woolly mammal tooth pits, buckles, and inside-out Beringia, Quaternary Science Reviews, 20 (2001), pp. 549-574.

[69] R. E. M. Hedges And L. M. Reynard, Nitrogen isotopes and the trophic level of humans in archaeology, Journal of Archaeological Science, 34 (2007), pp. 12401251.

[70] R. Hilborn And M. Mangel, The ecological detective: confronting models with data, vol. 28, Princeton Univ Pr, 1997. 
[71] R. D. Holt, Predation, apparent competition, and the structure of prey communities., Theoretical Population Biology, 12 (1977), pp. 197-129.

[72] D. M. Hopkins, Paleoecology of Beringia, Academic Press, New York, 1982.

[73] J. B. Hopkins And J. M. Ferguson, Estimating the diets of animals using stable isotopes and a comprehensive bayesian mixing model., PLoS ONE, 7 (2012), p. e28478.

[74] S. HubBell, The unified neutral theory of biodiversity and biogeography, Princeton Univ Press, 2001.

[75] V. A. A. Jansen And G. D. Kokkoris, Complexity and stability revisited, Ecol. Lett., 6 (2003), pp. 498-502.

[76] M. Janssen, J. Sept, And C. Griffith, Hominids Foraging in a Complex Landscape: Could Homo ergaster and Australopithecus boisei Meet Their Calories Requirements?, Takahashi, S., Sallach, D., \& and Rouchier, J.(Eds.), Advancing Social Simulation. Springer Publishing, (2007), pp. 307-318.

[77] J. Jeschke, M. Kopp, And R. TollRian, Consumer-food systems: why type I functional responses are exclusive to filter feeders, Biological Reviews, 79 (2004), pp. $337-349$.

[78] C. J. Jolly, The seed-eaters: a new model of hominid differentiation based on a baboon analogy, Man, 5 (1970), pp. 1-26. 
[79] S. R. Jones And K. C. KIM, Aculeus Wear and Oviposition in Four Species of Tephritidae (Diptera), Annals of the Entomological Society of America, 87 (1994), pp. 104-107.

[80] F. JuAnes, Why do decapod crustaceans prefer small-sized molluscan prey?, Marine Ecology Progress Series, 87 (1992), pp. 239-239.

[81] F. Juanes And L. Smith, The ecological consequences of limb damage and loss in decapod crustaceans: a review and prospectus, Journal of Experimental Marine Biology and Ecology, 193 (1995), pp. 197-223.

[82] R. F. KAY, The nut-crackers - a new theory of the adaptations of the Ramapithecinae, Am. J. Phys. Anthropol., 55 (1981), pp. 141-151.

[83] C. Keylock, Simpson diversity and the Shannon-Wiener index as special cases of a generalized entropy, Oikos, 109 (2005), pp. 203-207.

[84] P. L. KocH, Isotopic reconstruction of past continental environments, Annu. Rev. Earth. Pl. Sc., 26 (1998), pp. 573-613.

[85] _ Isotopic study of the biology of modern and fossil vertebrates, in Stable Isotopes in Ecology and Environmental Science., R. Michener and K. Lajtha, eds., Blackwell Publishing, Boston, 2007, pp. 99-154.

[86] P. L. Koch And A. Barnosky, Late Quaternary extinctions: state of the debate, Annu. Rev. Ecol. Evol. Syst., 37 (2006), pp. 215-250. 
[87] P. L. Koch And A. K. Behrensmeyer, The isotopic ecology of plants and animals in Amboseli National Park, Kenya, Annual Report to the Director, (1990).

[88] A. Kohn And A. Riggs, Sample Size Dependence in Measures of Proportional Similarity., Mar. Ecol.-Prog. Ser., 9 (1982), pp. 147-151.

[89] G. D. Kokkoris, V. A. A. Jansen, M. Loreau, and A. Y. Troumbis, Variability in interaction strength and implications for biodiversity, J. Anim. Ecol., 71 (2002), pp. 362-371.

[90] M. Kondoh, S. Kato, And Y. Sakato, Food webs are built up with nested subwebs, Ecology, 91 (2010), pp. 3123-3130.

[91] A. E. Krause, K. A. Frank, D. M. Mason, R. E. Ulanowicz, and W. W. TAYlor, Compartments revealed in food-web structure, Nature, 426 (2003), pp. 282-285.

[92] A. Krishna, P. R. Guimarães Jr, P. Jordano, and J. Bascompte, A neutral-niche theory of nestedness in mutualistic networks, Oikos, 117 (2008), pp. $1609-1618$.

[93] G. Laden And R. Wrangham, The rise of the hominids as an adaptive shift in fallback foods: Plant underground storage organs (USOs) and australopith origins, Journal of Human Evolution, 49 (2005), pp. 482-498.

[94] C. A. Layman, D. A. Arrington, C. G. Montaña, and D. M. Post, Can 
stable isotope ratios provide for community-wide measures of trophic structure?, Ecology, 88 (2007), pp. 42-48.

[95] J. A. Leonard, C. Vilà, K. Fox-Dobbs, P. L. Koch, R. K. Wayne, and B. Van Valkenburgh, Megafaunal Extinctions and the Disappearance of a Specialized Wolf Ecomorph, Current Biology, 17 (2007), pp. 1146-1150.

[96] W. Leonard And M. Robertson, Comparative primate energetics and hominid evolution, Am. J. Phys. Anthropol., 102 (1997), pp. 265-281.

[97] T. Levi, F. Lu, D. Yu, And M. Mangel, The behaviour and diet breadth of central-place foragers: an application to human hunters and Neotropical game management, Evolutionary Ecology Research, 13 (2011), pp. 171-185.

[98] S. L. Lindstedt And W. A. Calder III, Body size, physiological time, and longevity of homeothermic animals, Quarterly Review of Biology, 56 (1981), pp. 116.

[99] E. D. Lorenzen, D. Nogués-Bravo, L. Orlando, J. Weinstock, J. Binladen, K. A. Marske, A. Ugan, M. K. Borregaard, M. T. P. Gilbert, R. Nielsen, S. Y. W. Ho, T. Goebel, K. E. Graf, D. Byers, J. T. Stenderup, M. Rasmussen, P. F. Campos, J. A. Leonard, K.-P. Koepfli, D. Froese, G. Zazula, T. W. Stafford, K. Aaris-Sørensen, P. Batra, A. M. Haywood, J. S. Singarayer, P. J. Valdes, G. Boeskorov, J. A. Burns, S. P. Davydov, J. Haile, D. L. Jenkins, P. Kosintsev, 
T. Kuznetsova, X. Lai, L. D. Martin, H. G. McDonald, D. Mol, M. Meldgafrd, K. Munch, E. Stephan, M. Sablin, R. S. Sommer, T. Sipko, E. Scott, M. A. Suchard, A. Tikhonov, R. Willerslev, R. K. Wayne, A. Cooper, M. Hofreiter, A. Sher, B. Shapiro, C. Rahbek, AND E. Willerslev, Species-specific responses of Late Quaternary megafauna to climate and humans., Nature, 479 (2011), pp. 359-364.

[100] R. Lu, E. Smith, And I. Good, Multivariate measures of similarity and niche overlap., Theoretical Population Biology, 35 (1989), p. 1.

[101] S. Lubetkin And C. Simenstad, Multi-source mixing models to quantify food web sources and pathways, Journal of Applied Ecology, 41 (2004), pp. 996-1008.

[102] P. Lucas, P. Constantino, B. Wood, and B. Lawn, Dental enamel as a dietary indicator in mammals, Bioessays, 30 (2008), pp. 374-385.

[103] P. Lucas, I. Turner, N. Dominy, And N. Yamashita, Mechanical defences to herbivory, Ann Bot-London, 86 (2000), pp. 913-920.

[104] P. W. LucAs, Dental functional morphology: how teeth work, Cambridge Univ $\operatorname{Pr}, 2004$.

[105] P. W. Lucas, P. J. Constantino, and B. A. Wood, Inferences regarding the diet of extinct hominins: structural and functional trends in dental and mandibular morphology within the hominin clade, J Anatomy, 212 (2008), pp. 486-500. 
[106] R. MacArthur, Fluctuations of Animal Populations and a Measure of Community Stability, Ecology, 36 (1955), pp. 533-536.

[107] R. H. MacArthur and E. R. Pianka, On Optimal Use of a Patchy Environment, Am Nat, 100 (1966), pp. 603-+.

[108] G. Mасно, Effects of loading on the biochemical behavior of molars of Homo, Pan, and Pongo - Macho - 1999 - American Journal of Physical Anthropology Wiley Online Library, American journal of physical ..., (1999).

[109] M. Mangel And C. W. Clark, Dynamic modeling in behavioral ecology, Princeton Univ Pr, 1988.

[110] R. S. Manly, H. C. Hodge, And L. E. Ange, Density and Refractive Index Studies of Dental Hard Tissues: II. Density Distribution Curves 1,2, Journal of Dental Research, 18 (1939), pp. 203-211.

[111] F. W. Marlowe, A critical period for provisioning by Hadza men, Evolution and Human Behavior, 24 (2003), pp. 217-229.

[112] L. Martin, Significance of enamel thickness in hominoid evolution, Nature, 314 (1985), pp. 260-263.

[113] R. M. MaY, Will a Large Complex System be Stable?, Nature, 238 (1972), pp. 413414.

[114] C. McArthur And G. D. SAnson, Tooth wear in eastern grey kangaroos ( Macropus giganteus) and western grey kangaroos ( Macropus fuliginosus), and 
its potential influence on diet selection, digestion and population parameters, J. Zoology, 215 (1988), pp. 491-504.

[115] K. McCann, A. Hastings, And G. R. Huxel, Weak trophic interactions and the balance of nature, Nature, 395 (1998), pp. 794-798.

[116] M. L. MCKInNey, Extinction vulnerability and selectivity: Combining ecological and paleontological views, Annual Review of Ecology and Systematics, 28 (1997), pp. $495-516$.

[117] J. M. McNamara And A. I. Houston, Short-term behavior and lifetime fitness, in Functional Ontogeny, D. J. McFarland, ed., Pitman, London, 1982, pp. 60-87.

[118] C. J. Melián And J. Bascompte, Food web cohesion, Ecology, 85 (2004), pp. $352-358$.

[119] P. MeLlars, Neanderthals and the modern human colonization of Europe., Nature, 432 (2004), pp. 461-465.

[120] B. Milakovic And K. L. Parker, Using stable isotopes to define diets of wolves in northern British Columbia, Canada, Journal of Mammalogy, 92 (2011), pp. 295-304.

[121] K. Milton, Primate diets and gut morphology: implications for hominid evolution, in Food and evolution: toward a theory of human food habits, Temple Univ $\operatorname{Pr}, 1989$. 
[122] J. W. Moore And B. X. Semmens, Incorporating uncertainty and prior information into stable isotope mixing models, Ecol. Lett., 11 (2008), pp. 470-480.

[123] M. Morisita, Measuring of interspecific association and similarity between communities, Memoirs of the Faculty of Science, Kyushu University, Series E (Biology), 3 (1959), pp. 65-80.

[124] D. Muhs, T. Ager, And J. Begét, Vegetation and paleoclimate of the last interglacial period, central Alaska, Quaternary Science Reviews, 20 (2001), pp. 4161.

[125] W. Murdoch, The functional response of predators, Journal of Applied Ecology, 10 (1973), pp. 335-342.

[126] S. Newsome, P. Collins, T. Rick, D. Guthrie, J. Erlandson, and M. FoGEL, Pleistocene to historic shifts in bald eagle diets on the Channel Islands, California, Proc. Natl. Acad. Sci. USA, 107 (2010), p. 9246.

[127] S. Newsome, J. D. Yeakel, P. V. Wheatley, and M. T. Tinker, Tools for quantifying isotopic niche space and dietary variation at the individual and population level, Journal of Mammalogy, 93 (2012), pp. 329-341.

[128] S. D. Newsome, C. Martinez del Rio, S. Bearhop, and D. L. Phillips, A niche for isotopic ecology, Front. Ecol. Environ., 5 (2007), pp. 429-436.

[129] M. NovaK, Trophic omnivory and the structure, strength, and nonlinear nature 
of species interactions across a productivity gradient, PhD Dissertation, (2008), pp. $1-277$.

[130] M. NovAK, Estimating interaction strengths in nature: experimental support for an observational approach, Ecology, 91 (2010), pp. 2394-2405.

[131] M. Novak And J. T. Wootton, Estimating nonlinear interaction strengths: An observation-based method for species-rich food webs, Ecology, 89 (2008), pp. 20832089.

[132] M. Novak, J. T. Wootton, D. Doak, M. Emmerson, J. A. Estes, And M. T. TINKER, Predicting community responses to perturbations in the face of imperfect knowledge and network complexity, Ecology, 92 (2011), pp. 836-846.

[133] H. OKARma, The trophic ecology of wolves and their predatory role in ungulate communities of forest ecosystems in Europe, Y Acta Theriologica, 40 (1995).

[134] M. H. O'Leary, Carbon isotopes in photosynthesis, Bioscience, (1988), pp. 328336.

[135] J. M. Olesen, J. Bascompte, Y. L. Dupont, H. Elberling, C. Rasmussen, And P. Jordano, Missing and forbidden links in mutualistic networks, Proc. Roy. Soc. B, (2010), pp. 1-18.

[136] J. W. Osborne, Ageing, in Dental Anatomy and Embryology Vol. 2: A comparison to dental studies, A. H. R. Rowe and R. B. Johns, eds., Blackwell, Oxford, 1981, pp. 352-356. 
[137] R. T. PAInE, Food webs: linkage, interaction strength and community infrastructure, The Journal of Animal Ecology, 49 (1980), pp. 666-685.

[138] — Road Maps of Interactions or Grist for Theoretical Development?, Ecology, 69 (1988), pp. 1648-1654.

[139] A. C. Parnell, R. Inger, S. Bearhop, and A. L. Jackson, Source Partitioning Using Stable Isotopes: Coping with Too Much Variation, PLoS ONE, 5 (2010), p. e9672.

[140] M. Pascual And J. Dunne, Ecological networks: linking structure to dynamics in food webs, Oxford University Press, USA, 2006.

[141] M. Pascual And J. A. Dunne, Challenges for the future: integrating ecological structure and dynamics, in Ecological networks: linking structure to dynamics in food webs, Oxford University Press, USA, 2006, pp. 351-373.

[142] J. S. Pate And K. W. Dixon, Tuberous, cormous and bulbous plants. Biology of an adaptive strategy in Western Australia, Nedlands, Australia: University of Western Australia Press., (1982).

[143] F. J. Perez-Barberia And I. J. Gordon, The influence of molar occlusal surface area on the voluntary intake, digestion, chewing behaviour and diet selection of red deer (Cervus elaphus), J. Zoology, 245 (1998), pp. 307-316.

[144] D. L. Phillips And J. W. GregG, Source partitioning using stable isotopes: coping with too many sources, Oecologia, 136 (2003), pp. 261-269. 
[145] D. L. Phillips And P. Koch, Incorporating concentration dependence in stable isotope mixing models, Oecologia, 130 (2002), pp. 114-125.

[146] D. L. Phillips, S. D. Newsome, And J. W. GregG, Combining sources in stable isotope mixing models: alternative methods, Oecologia, 144 (2005), pp. 520527.

[147] E. R. Pianka, Niche overlap and diffuse competition, Proc. Natl. Acad. Sci. USA, 71 (1974), pp. 2141-2145.

[148] M. M. Pires, P. I. Prado, and P. R. Guimarães Jr, Do Food Web Models Reproduce the Structure of Mutualistic Networks?, PLoS ONE, 6 (2011), p. e27280.

[149] Ş. Procheş, R. M. Cowling, P. Goldblatt, J. C. Manning, and D. A. Snijman, An overview of the Cape geophytes, Biol J Linn Soc, 87 (2006), pp. 2743.

[150] D. Rabenold and O. M. Pearson, Abrasive, Silica Phytoliths and the Evolution of Thick Molar Enamel in Primates, with Implications for the Diet of Paranthropus boisei, PLoS ONE, 6 (2011), p. e28379.

[151] P. Raia, F. Carotenuto, F. Passaro, D. Fulgione, and M. Fortelius, Ecological Specialization in Fossil Mammals Explains Cope's Rule, Am Nat, 179 (2012), pp. 328-337.

[152] W. Research, Mathematica 8.0, Wolfram Research, Inc, Champaign, Illinois, version 8.0 ed., 2010. 
[153] M. Richards And E. Trinkaus, Isotopic evidence for the diets of European Neanderthals and early modern humans, Proc. Natl. Acad. Sci. USA, 106 (2009), p. 16034.

[154] W. J. Ripple And T. P. Rooney, Large predators, deer, and trophic cascades in boreal and temperate ecosystems, in Trophic Cascades, Island Pr, Apr. 2010.

[155] F. Rivals, M. C. Mihlbachler, N. Solounias, D. Mol, G. M. Semprebon, J. De Vos, And D. C. Kalthoff, Palaeoecology of the Mammoth Steppe fauna from the late Pleistocene of the North Sea and Alaska: Separating species preferences from geographic influence in paleoecological dental wear analysis, Palaeogeogr Palaeocl, 286 (2010), pp. 42-54.

[156] C. T. Robbins, L. A. Felicetti, And S. T. Florin, The impact of protein quality on stable nitrogen isotope ratio discrimination and assimilated diet estimation, Oecologia, 162 (2010), pp. 571-579.

[157] C. T. Robbins, L. A. Felicetti, And M. Sponheimer, The effect of dietary protein quality on nitrogen isotope discrimination in mammals and birds., Oecologia, 144 (2005), pp. 534-540.

[158] B. D. Roitberg, D. R. Gillespie, D. M. J. Quiring, C. R. Alma, W. H. Jenner, J. Perry, J. H. Peterson, M. Salomon, and S. Vanlaerhoven, The cost of being an omnivore: mandible wear from plant feeding in a true bug., Naturwissenschaften, 92 (2005), pp. 431-434. 
[159] J. D. Roth And K. A. Hobson, Stable carbon and nitrogen isotopic fractionation between diet and tissue of captive red fox: implications for dietary reconstruction, Can. J. Zool., 78 (2000), pp. 848-852.

[160] J. T. Roughgarden, Evolution of Niche Width, Am Nat, 106 (1972), pp. 683718.

[161] K. Sayers, M. A. Norconk, and N. L. Conklin-Brittain, Optimal foraging on the roof of the world: Himalayan langurs and the classical prey model., Am. J. Phys. Anthropol., 141 (2010), pp. 337-357.

[162] G. Schwartz, J. Thackeray, C. Reid, and J. van Reenan, Enamel thickness and the topography of the enamel-dentine junction in South African PlioPleistocene hominids with special reference to the Carabelli trait, in J Hum Evol, Univ London Univ Coll, Dept Anat \& Dev Biol, Evolut Anat Unit, London WC1E 6BT, England, 1998, pp. 523-542.

[163] B. X. Semmens, E. J. Ward, J. W. Moore, and C. T. Darimont, Quantifying inter-and intra-population niche variability using hierarchical Bayesian stable isotope mixing models, PLoS ONE, 4 (2009), p. e6187.

[164] B. Shapiro, A. Drummond, A. Rambaut, M. Wilson, P. Matheus, A. Sher, O. Pybus, M. Gilbert, I. Barnes, J. Binladen, E. Willerslev, A. J. Hansen, G. F. Baryshnikov, J. A. Burns, S. Davydov, J. C. Driver, D. G. Froese, C. R. Harnington, G. Keddie, P. Kosintsev, 
M. L. Kunz, L. D. Martin, R. O. Stephenson, J. Storer, R. Tedford, S. Zimov, And A. Cooper, Rise and fall of the Beringian steppe bison, Science, 306 (2004), p. 1561.

[165] R. P. Shellis, A. D. Beynon, D. J. Reid, and K. M. Hitemae, Variations in molar enamel thickness among primates., Journal of Human Evolution, 35 (1998), pp. 507-522.

[166] A. R. E. Sinclair, S. Mduma, And J. S. Brashares, Patterns of predation in a diverse predator-prey system, Nature, 425 (2003), pp. 288-290.

[167] T. Skogland, Tooth wear by food limitation and its life history consequences in wild reindeer, Oikos, 51 (1988), pp. 238-242.

[168] N. Slade AND S. BlaIR, An empirical test of using counts of individuals captured as indices of population size, Journal of Mammalogy, 81 (2000), pp. 1035-1045.

[169] E. Smith, K. Pontasch, And J. Cairns, Community similarity and the analysis of multispecies environmental data: a unified statistical approach, Water Res., 24 (1990), pp. 507-514.

[170] T. M. Smith, A. J. Olejniczak, L. B. Martin, And D. J. Reid, Variation in hominoid molar enamel thickness., Journal of Human Evolution, 48 (2005), pp. $575-592$.

[171] R. SolÉ And J. BASCOMPte, Self-organization in complex ecosystems, Princeton Univ Pr, 2006. 
[172] R. V. SolÉ And J. M. Montoya, Complexity and fragility in ecological networks, Proc. Roy. Soc. B, 268 (2001), pp. 2039-2045.

[173] R. V. Solé, J. Saldaña, J. M. Montoya, and D. H. Erwin, Simple model of recovery dynamics after mass extinction, Journal of Theoretical Biology, 267 (2010), pp. 193-200.

[174] R. L. Spaulding, P. R. Krausman, and W. B. Ballard, Summer diet of Gray Wolves, Canis lupus, in northwestern Alaska, Canadian Field Naturalist, 112 (1998), pp. 262-266.

[175] M. Sponheimer And J. LeE-Thorp, Isotopic evidence for the diet of an early hominid, Australopithecus africanus, Science, 283 (1999), p. 368.

[176] M. Sponheimer, J. Lee-Thorp, D. De Ruiter, D. Codron, J. Codron, A. Baugh, And F. Thackeray, Hominins, sedges, and termites: new carbon isotope data from the Sterkfontein valley and Kruger National Park, Journal of Human Evolution, 48 (2005), pp. 301-312.

[177] I. StIRLING, Tooth wear as a mortality factor in the Weddell seal, Leptonychotes weddelli, Journal of Mammalogy, 50 (1969), pp. 559-565.

[178] D. B. Stouffer And J. BAscompte, Understanding food-web persistence from local to global scales, Ecol. Lett., 13 (2010), pp. 154-161.

[179] D. B. Stouffer And J. Bascompte, Compartmentalization increases food-web persistence, Proc. Natl. Acad. Sci. USA, 108 (2011), pp. 3648-3652. 
[180] D. B. Stouffer, E. L. Rezende, And L. A. Nunes Amaral, the role of body mass in diet contiguity and food-web structure, J. Anim. Ecol., 80 (2011), pp. 632-639.

[181] D. S. Strait, G. W. Weber, S. Neubauer, J. Chalk, B. G. Richmond, P. W. Luchi, M. A. Spencer, C. Schrein, P. C. Dechow, C. F. Ross, I. R. Grosse, B. W. Wright, P. Constantino, B. A. Wood, B. Lawn, W. L. Hylander, Q. Wang, C. Byron, D. E. Slice, and A. L. Smith, The feeding biomechanics and dietary ecology of Australopithecus africanus, Proc. Natl. Acad. Sci. USA, 106 (2009), pp. 2085-2086.

[182] C. Swennen, L. De Bruijn, P. Duiven, M. Leopold, and E. Marteijn, Differences in bill form of the oystercatcher haematopus ostralegus; a dynamic adaptation to specific foraging techniques, Netherlands Journal of Sea Research, 17 (1983), pp. 57-83.

[183] K. TAnabe And T. NAmba, Omnivory creates chaos in simple food web models, Ecology, 86 (2008), pp. 3411-3414.

[184] E. ThÉbault And C. Fontaine, Stability of Ecological Communities and the Architecture of Mutualistic and Trophic Networks, Science, 329 (2010), pp. 853856.

[185] I. Thompson and P. Colgan, Prey choice by marten during a decline in prey abundance, Oecologia, 83 (1990), pp. 443-451. 
[186] D. Tilman, R. M. May, C. Lehman, and M. Nowak, Habitat destruction and the extinction debt, Nature, 371 (1994), pp. 65-66.

[187] M. T. Tinker, P. R. Guimarães JR, M. Novak, F. M. D. Marquitti, B. J, S. M, G. Bentall, And J. A. Estes, Structure and mechanism of diet specialization: testing models of individual variation in resource use with sea otters, Ecol. Lett., in press (2012).

[188] J. M. Tylianakis, E. Laliberté, A. Nielsen, and J. Bascompte, Conservation of species interaction networks, Biol. Conserv., 143 (2010), pp. 2270-2279.

[189] P. S. Ungar, F. E. Grine, And M. F. Teaford, Dental Microwear and Diet of the Plio-Pleistocene Hominin Paranthropus boisei, PLoS ONE, 3 (2008), p. e2044.

[190] P. S. Ungar, R. S. Scott, F. E. Grine, and M. F. Teaford, Molar microwear textures and the diets of Australopithecus anamensis and Australopithecus afarensis, Philosophical Transactions of the Royal Society B: Biological Sciences, 365 (2010), pp. 3345-3354.

[191] P. S. Ungar And M. Sponheimer, The Diets of Early Hominins, Science, 334 (2011), pp. 190-193.

[192] E. J. M. URTON AND K. A. Hobson, Intrapopulation variation in gray wolf isotope $\left(\delta^{15} \mathrm{~N}\right.$ and $\left.\delta^{13} \mathrm{C}\right)$ profiles: implications for the ecology of individuals, Oecologia, 145 (2005), pp. 317-326. 
[193] B. van Valkenburgh, X. Wang, and J. Damuth, Cope's rule, hypercarnivory, and extinction in North American canids, Science, 306 (2004), p. 101.

[194] M. VAnder Zanden And J. Rasmussen, Variation in $\delta^{15} \mathrm{~N}$ and $\delta^{13} \mathrm{C}$ trophic fractionation: implications for aquatic food web studies, Limnology and Oceanography, (2001), pp. 2061-2066.

[195] A. S. Vincent, Plant foods in savanna environments: a preliminary report of tubers eaten by the Hadza of Northern Tanzania., World Archaeology, 17 (1985), pp. $131-148$.

[196] J. WeInstock, The Upper Pleistocene mammalian fauna from the Große Grotte near Blaubeuren (southwestern Germany), Geologie und Paläontologie 277, 1e49. Stuttgarter Beiträge zur Naturkunde, Serie B., (1999).

[197] D. Weishampel, P. Dodson, and H. Osmólska, eds., The Dinosauria, Univ of California $\operatorname{Pr}, 2004$.

[198] R. Williams, E. L. Berlow, J. A. Dunne, A. Barabási, and N. Martinez, Two degrees of separation in complex food webs, Proc. Natl. Acad. Sci. USA, 99 (2002), p. 12913.

[199] S. H. Williams, B. W. Wright, V. D. Truong, C. R. Daubert, and C. J. VINYARD, Mechanical properties of foods used in experimental studies of primate masticatory function, Am. J. Primatol., 67 (2005), pp. 329-346.

[200] K. Willis And T. VAn Andel, Trees or no trees? The environments of central 
and eastern Europe during the Last Glaciation, Quaternary Science Reviews, 23 (2004), pp. 2369-2387.

[201] B. A. Wood And P. J. Constantino, Paranthropus boisei: Fifty years of evidence and analysis, Am. J. Phys. Anthropol., 134 (2007), pp. 106-132.

[202] G. Woodward And A. G. Hildrew, Body-size determinants of niche overlap and intraguild predation within a complex food web, J. Anim. Ecol., 71 (2002), pp. 1063-1074.

[203] J. T. Wootton And M. Emmerson, Measurement of interaction strength in nature, Annu. Rev. Ecol. Evol. Syst., 36 (2005), pp. 419-444.

[204] J. D. Yeakel, N. C. Bennett, P. L. Koch, and N. J. Dominy, The isotopic ecology of African mole rats informs hypotheses on the evolution of human diet, Proc. Roy. Soc. B, 274 (2007), pp. 1723-1730.

[205] J. D. Yeakel, P. R. Guimarães JR, M. Novak, K. Fox-Dobbs, and P. L. KocH, Probabilistic patterns of interaction: The effects of link-strength variance on food-web structure, Preprint, (2012).

[206] J. D. Yeakel, M. Novak, P. R. Guimarães JR, N. J. Dominy, P. L. Koch, E. J. Ward, J. W. Moore, And B. X. Semmens, Merging Resource Availability with Isotope Mixing Models: The Role of Neutral Interaction Assumptions, PLoS ONE, 6 (2011), p. e22015. 
[207] J. D. Yeakel, B. D. Patterson, K. Fox-Dobbs, M. Okumura, T. CerLIng, J. Moore, P. Koch, And N. Dominy, Cooperation and individuality among man-eating lions, Proc. Natl. Acad. Sci. USA, 106 (2009), pp. 19040-19043.

[208] B. Yurtsev, The Pleistocene "Tundra-Steppe" and the productivity paradox: the landscape approach, Quaternary Science Reviews, 20 (2001), pp. 165-174.

[209] G. D. Zazula, D. G. Froese, C. E. Schweger, R. W. Mathewes, A. B. Beaudoin, A. M. Telka, C. R. Harington, And J. A. Westgate, Ice-age steppe vegetation in east Beringia., Nature, 423 (2003), p. 603.

[210] G. D. Zazula, C. E. Schweger, A. B. Beaudoin, and G. H. McCourt, Macrofossil and pollen evidence for full-glacial steppe within an ecological mosaic along the Bluefish River, eastern Beringia, Quaternary International, 142-143 (2006), pp. $2-19$.

[211] P. Zhang, J. Wang, X. Li, M. Li, Z. Di, And Y. FAn, Clustering coefficient and community structure of bipartite networks, Physica A, 387 (2008), pp. 68696875. 